\title{
Performance of the Atlas Forward Calorimeter and Search for the Invisible Higgs via Vector Boson Fusion at ATLAS
}

\author{
by
}

\section{Malachi Schram}

\author{
A Dissertation submitted to \\ the Faculty of Graduate Studies and Research \\ in partial fulfilment of \\ the requirements for the degree of \\ Doctor of Philosophy
}

Ottawa-Carleton Institute of Physics

\author{
Department of Physics \\ Carleton University \\ Ottawa, Ontario, Canada \\ April 2008 \\ Copyright (C) \\ 2008 Malachi Schram
}




$\begin{array}{ll}\begin{array}{l}\text { Library and } \\ \text { Archives Canada }\end{array} & \begin{array}{l}\text { Bibliothèque et } \\ \text { Archives Canada }\end{array} \\ \begin{array}{l}\text { Published Heritage } \\ \text { Branch }\end{array} & \begin{array}{l}\text { Direction du } \\ \text { Patrimoine de l'édition }\end{array} \\ \begin{array}{l}\text { 395 Wellington Street } \\ \text { Ottawa ON K1A 0N4 } \\ \text { Canada }\end{array} & \begin{array}{l}\text { 395, rue Wellington } \\ \text { Ottawa ON K1A 0N4 } \\ \text { Canada }\end{array}\end{array}$

Your file Votre référence ISBN: 978-0-494-40536-9 Our file Notre référence ISBN: 978-0-494-40536-9

NOTICE:

The author has granted a nonexclusive license allowing Library and Archives Canada to reproduce, publish, archive, preserve, conserve, communicate to the public by telecommunication or on the Internet, loan, distribute and sell theses worldwide, for commercial or noncommercial purposes, in microform, paper, electronic and/or any other formats.

The author retains copyright ownership and moral rights in this thesis. Neither the thesis nor substantial extracts from it may be printed or otherwise reproduced without the author's permission.
AVIS:

L'auteur a accordé une licence non exclusive permettant à la Bibliothèque et Archives Canada de reproduire, publier, archiver, sauvegarder, conserver, transmettre au public par télécommunication ou par l'Internet, prêter, distribuer et vendre des thèses partout dans le monde, à des fins commerciales ou autres, sur support microforme, papier, électronique et/ou autres formats.

L'auteur conserve la propriété du droit d'auteur et des droits moraux qui protège cette thèse. $\mathrm{Ni}$ la thèse ni des extraits substantiels de celle-ci ne doivent être imprimés ou autrement reproduits sans son autorisation.
In compliance with the Canadian Privacy Act some supporting forms may have been removed from this thesis.

While these forms may be included in the document page count, their removal does not represent any loss of content from the thesis.
Conformément à la loi canadienne sur la protection de la vie privée, quelques formulaires secondaires ont été enlevés de cette thèse.

Bien que ces formulaires aient inclus dans la pagination, il n'y aura aucun contenu manquant.

\section{Canada}




\begin{abstract}
The ATLAS detector will examine proton-proton collisions at $14 \mathrm{TeV}$ provided by CERN's Large Hadron Collider (LHC). ATLAS is a general purpose detector with tracking, calorimetry and a large muon system. The calorimeter system provides hermetic coverage of a large fraction of the solid angle of the detector. In the region close to the beam line, the calorimeter components are the FCal detectors which provide additional $\eta$ coverage improving the jet tagging efficiency and the missing energy resolution. The performance of the FCal calorimeter for both electrons and hadrons is one of the major topics of this thesis. The measured electromagnetic response for the FCal 1 module was $12.14 \pm 0.06$ $\mathrm{ADC} / \mathrm{GeV}$ which is in good agreement with the predicted value of $12 \mathrm{ADC} / \mathrm{GeV}$ from the simulation which will be used to provide the initial electromagnetic response for the FCal modules during the early stages of ATLAS data taking. The hadronic performance was investigated using two calibration schemes: flat weights and transverse weights. The energy resolution for hadrons using transverse weights was found to be $\sigma_{E} / E=(72.7 \pm 1.5) \% / \sqrt{E_{b}} \oplus(2.85 \pm 0.28) \%$, here $E_{b}$ is in $\mathrm{GeV}$, exceeding design requirements. In addition to good resolution, the transverse weights provided a uniform energy response, within $\sim 3 \%$, for energies greater than $40 \mathrm{GeV}$.
\end{abstract}

In some extensions of the Standard Model the Higgs may predominately decay to particles not visible in the detector. An upper limit on the sensitivity to observe an invisible Higgs with the ATLAS detector via the vector boson fusion production channel was investigated. Two complementary studies were undertaken: a signal-to-background analysis and a shape analysis. The results from the signal-to-background analysis using $30 \mathrm{fb}^{-1}$ of data for a $130 \mathrm{GeV}$ mass Higgs show that it is possible to detect an invisible Higgs if the beyond the Standard Model cross-section is greater than $\sim 72 \%$ of the Standard Model cross-section at $99 \%$ confidence level assuming a $100 \%$ Higgs branching ratio to invisible particles. For the shape analysis the sensitivity is $\sim 104 \%$. The uncertainty in the jet energy scale and resolution are the dominant systematic errors in these studies. 


\section{Acknowledgements}

The biggest reason why I am writing this thank you list today is for my wonderful wife, Michelle, for her help and support. She has been there from the very beginning encouraging me and providing a positive outlook at every step. Our family has grown to include two wonderful children, Maya and Kody. They have given me so much without ever having to say a word. Maya's energy and curiosity inspires me everyday and Kody's smile shows me the endless possibilities. I would like to also thank my parents and my in-laws for their endless support throughout this adventure.

In addition to my family, there are a number of people to thank, however, none deserve more acknowledgement that my adviser, Gerald Oakham. Gerald has had a pivotal influence on my research with his guidance and patience. I would also like to thank the Carleton ATLAS Group, the Carleton Physics Department and the Carleton Physics Computing Group for their amazing support through the process of this thesis.

I would like to thank the ATLAS Canada group, specifically Peter Krieger for numerous helpful discussions on the 2003 test beam and other subjects. I wish to thank the Arizona ATLAS Group, specifically Peter Loch for his time and suggestions on hadronic calibration and physics topics.

Thanks to the Higgs Working Group and the ATLAS Physics Coordinator for the "lively" discussions on the Invisible Higgs. Furthermore, special thanks to Pauline Gagnon for the productive discussions on the Invisible Higgs analysis and the CSC Note. Thank you to CERN for providing the resources required to conduct my analysis.

Finally, many thanks to Marco and Warren for joining me on my "escape" trips. 


\section{Contents}

$\begin{array}{ll}\text { Abstract } & \text { ii }\end{array}$

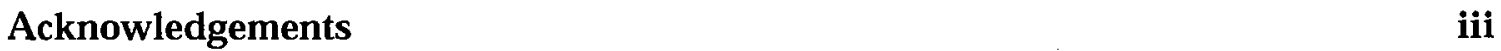

List of Tables $\quad$ vii

List of Figures $\quad$ xi

1 Introduction 1

2 LHC and the ATLAS Experiment 6

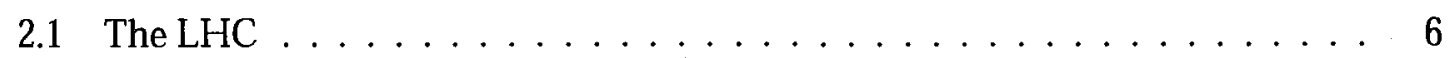

2.2 The ATLAS Experiment $\ldots \ldots \ldots \ldots \ldots \ldots$

2.3 The ATLAS Coordinate System . . . . . . . . . . . . . . 8

2.4 The ATLAS Inner Detector . . . . . . . . . . . . . . . . . 9

2.5 The ATLAS Calorimeters . . . . . . . . . . . . . . . . . 11

2.6 The ATLAS Muon System . . . . . . . . . . . . . . . . . . . 14

2.7 Trigger and Data Acquisition $\ldots \ldots \ldots \ldots \ldots \ldots$

3 Principles of Shower Process and Calorimetry 19

3.1 Shower Processes . . . . . . . . . . . . . . . . . . . . . 19

3.1.1 Electromagnetic Showers . . . . . . . . . . . . . . 20

3.1 .2 Hadronic Showers . . . . . . . . . . . . . . . . . . 23 


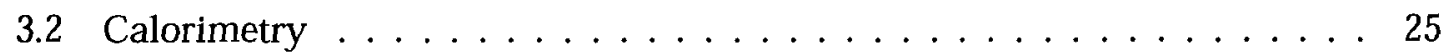

3.2.1 Sampling Calorimetry and Sampling Fraction $\ldots \ldots \ldots \ldots .25$

3.2 .2 Energy Resolution . . . . . . . . . . . . . . . . 27

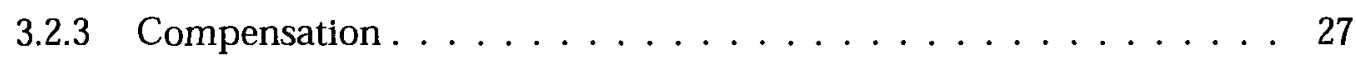

I The ATLAS Forward Calorimeter 2003 Test Beam 29

4 Introduction $\quad 30$

4.1 Motivation for the ATLAS FCal Modules $\ldots \ldots \ldots \ldots$. . . . . 30

4.2 The ATLAS FCal Calorimeter Design . . . . . . . . . . . . . . . . . 32

4.3 Test Beam Goals and Setup $\ldots \ldots \ldots \ldots$. . . . . . . . . 37

4.4 Beamline Instrumentation $\ldots \ldots \ldots \ldots$. . . . . . . . . 40

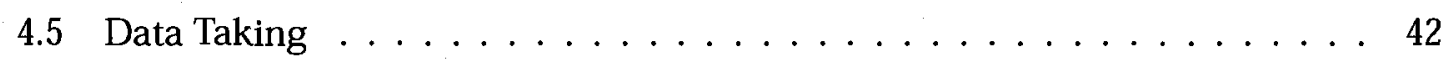

5 Data Treatment 44

5.1 Pedestal Stability $\ldots \ldots \ldots \ldots \ldots \ldots \ldots \ldots \ldots \ldots$

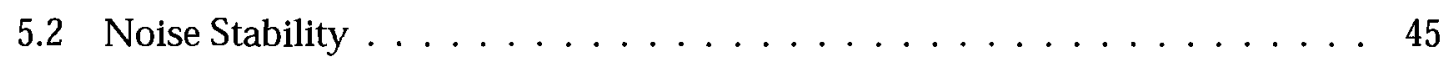

6 Calorimeter Amplitude Reconstruction 48

6.1 Introduction $\ldots \ldots \ldots \ldots \ldots \ldots \ldots \ldots \ldots \ldots \ldots$

6.2 Test Beam Event Phase . . . . . . . . . . . . . . . . . 49

6.3 Optimal Filtering Technique . . . . . . . . . . . . . . . . 50

6.3 .1 Auto-Correlation Matrix . . . . . . . . . . . . . . . . 53

6.3 .2 Pulse Shape Analysis . . . . . . . . . . . . . . . . . . 53

6.3.3 Calibration and Validation of the Optimal Filtering Coefficients . . . 55

7 Test Beam Results $\quad 60$

7.1 Beam Cleaning and Envelope Cuts . . . . . . . . . . . . . 60

7.2 Tracking $\ldots \ldots \ldots \ldots \ldots \ldots \ldots \ldots \ldots \ldots \ldots \ldots \ldots \ldots \ldots \ldots \ldots \ldots$

7.2.1 Calorimeter Based Transverse Shower Position Calculation . . . . . 62 
7.2.2 Tracking and Calorimeter Alignment . . . . . . . . . . . . 64

7.2.3 Stability of Corrected Tracking Shifts . . . . . . . . . . . . . . 65

7.3 Clustering and Noise $\ldots \ldots \ldots \ldots \ldots \ldots \ldots \ldots$

7.4 Electron Performance . . . . . . . . . . . . . . . . . 69

7.4 .1 Electron Energy Linearity . . . . . . . . . . . . . 70

7.4 .2 Electron Energy Resolution . . . . . . . . . . . . . . . . 74

7.5 Hadron Performance $\ldots \ldots \ldots \ldots \ldots \ldots$

7.5 .1 Compensation Techniques . . . . . . . . . . . . 77

7.5 .2 Baseline Approach $\ldots \ldots \ldots \ldots \ldots$

7.5 .3 Transverse Weights . . . . . . . . . . . . . . . . . 80

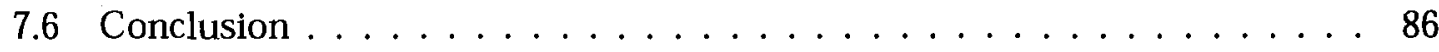

II ATLAS Sensitivity to an Invisible Higgs via Vector Boson Fusion $\quad 89$

8 Introduction $\quad 90$

8.1 The Standard Model and the Higgs boson . . . . . . . . . . . . . . . . 91

8.2 Phenomenology of the Standard Model Higgs . . . . . . . . . . . . . . 92

8.2.1 Constraints on the Standard Model Higgs Mass . . . . . . . . . . 93

8.2.2 Higgs Production and Branching Ratio at the LHC . . . . . . . . 96

8.3 Physics Beyond the Standard Model and the Invisible Higgs . . . . . . . 97

8.4 Status of Invisible Higgs Search . . . . . . . . . . . . . . . . . 101

9 Monte-Carlo Event Generators and Data Production $\quad 106$

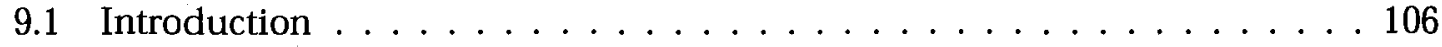

9.2 Event Generators . . . . . . . . . . . . . . . . . . . 108

9.2 .1 Herwig/Jimmy . . . . . . . . . . . . . . . . . . . 109

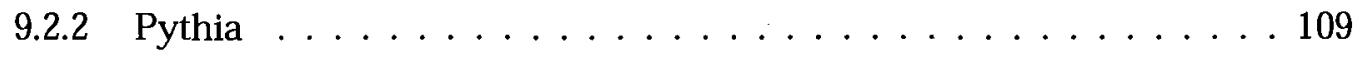

9.2 .3 Alpgen ............................. 110

9.3 Signal and Background Event Production . . . . . . . . . . . 111 
9.3.1 Invisible Higgs Signal $\ldots \ldots \ldots \ldots \ldots \ldots$

9.3.2 The QCD Dijets Background … . . . . . . . . . . 113

9.3.3 The $\mathrm{Z}+$ jets Background $\ldots \ldots \ldots \ldots$

9.3.4 The $\mathrm{W}+$ jets Background $\ldots \ldots \ldots \ldots \ldots \ldots$

9.4 Detector Simulation . . . . . . . . . . . . . . . . . . . . . . 118

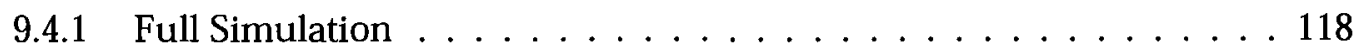

9.4 .2 Fast Simulation . . . . . . . . . . . . . . . . . . . . 119

9.5 Atlas Reconstruction . . . . . . . . . . . . . . . . . . . . 120

9.5.1 Electron Reconstruction . . . . . . . . . . . . . . . . 120

9.5.2 Muon Reconstruction . . . . . . . . . . . . . . . . . 121

9.5.3 Hadronic Tau Reconstruction . . . . . . . . . . . . . . . . 121

9.5.4 Jet Reconstruction . . . . . . . . . . . . . . . . . . 123

9.5.5 Missing Transverse Energy Reconstruction . . . . . . . . . . . 124

9.5 .6 Event Building $\ldots \ldots \ldots \ldots \ldots \ldots \ldots \ldots$

10 Sensitivity Study for an Invisible Higgs via the Vector Boson Fusion Channel 127

10.1 Analysis Framework . . . . . . . . . . . . . . . . . . . 128

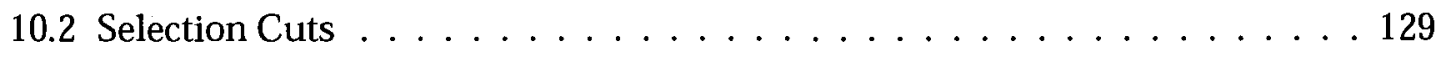

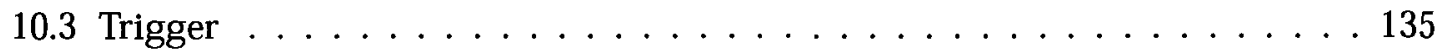

10.4 Signal-To-Background Analysis . . . . . . . . . . . . . . . . . 137

10.5 Shape Analysis . . . . . . . . . . . . . . . . . . . . . . 142

10.6 Systematic Uncertainties . . . . . . . . . . . . . . . . . . . 143

10.6.1 Event Generator Systematics ． . . . . . . . . . . . . . 145

10.6.2 Reconstruction Systematics . . . . . . . . . . . . . . 146

10.7 Conclusion . . . . . . . . . . . . . . . . . . . . 147

11 Conclusion $\quad 149$

$\begin{array}{ll}\text { Bibiography } & 151\end{array}$ 


\section{List of Tables}

2.1 Summary of the ATLAS detector performance goals. The units for energy, $\mathrm{E}$, and transverse momentum, $p_{T}$, are in $\mathrm{GeV}$ in the particle physics system, where $c$ and $\hbar$ are set to 1 . The variable, $\eta$, is defined in Section 2.3. . . . . 8

4.1 The ATLAS FCal detector geometrical properties and mass. The dimensions are for the detector at LAr temperature $(\sim 90 \mathrm{~K})$. Here $X_{0}$ and $\lambda$ are the radiation and nuclear interaction lengths, respectively. . . . . . . . . 34

4.2 The beam impact points and angles used in the beam test along with the equivalent $|\eta|$ angle in ATLAS. . . . . . . . . . . . . . . . . 39

6.1 Physics pulse shape analysis data summary. . . . . . . . . . . . . 54

6.2 Physics pulse shape $\chi^{2}$ goodness-of-fit summary from the analysis comparing the two pulse shape analysis methods. Here $\nu$ is the number of degrees of freedom and $\chi_{95 \%}^{2}$ is the $\chi^{2}$ function for $\nu$ degrees of freedom at a $95 \%$ confidence level. . . . . . . . . . . . . . . . . . . . 55

7.1 Effect of significance cuts on the mean center-of-gravity position for 100 $\mathrm{GeV}$ electron shower at the $4 \mathrm{~L}$ position. . . . . . . . . . . . . . . . 63

7.2 Effect of significance cuts on track shifts for $100 \mathrm{GeV}$ electron beam at the

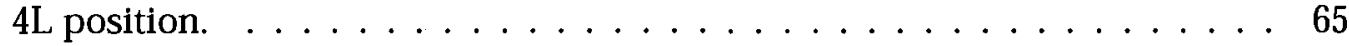

7.3 Corrected shifts for various electron beam runs at the $4 \mathrm{~L}$ position. . . . . . 67 
7.4 Cluster size results for the electron reconstructed energy. Columns 2-4 are the reconstructed energy in ADC for three diffrent cluster size. Column 5 is the average of the means and columns 6-7 are the rms of the means. . . . 72

7.5 Electron energy linearity for three different cylinder cluster size. . . . . . . 74

7.6 Results of the effects of cluster size on electron energy resolution. Column 2-4 are the corrected energy resolution for three different cluster sizes. . . . 75

7.7 Electron energy resolution for three different cluster size. . . . . . . . . 75

7.8 Results from flat weight fits with and without beam energy constraints. . . 80

7.9 Values determined for the stochastic and constant terms using different sets of flat weights derived with and without beam energy constraints. . . . . . 80

7.10 Values determined for the stochastic and constant terms using different sets of transverse weights derived without beam energy constraints from 120 $\mathrm{GeV}, 200 \mathrm{GeV}$ pion data, and the average of weights from $120,150,200 \mathrm{GeV}$ pion data. . . . . . . . . . . . . . . . . 83

7.11 Flat weights that are used to give beam energy constraints to the transverse weights obtained with $200 \mathrm{GeV}$ pion data. . . . . . . . . . . . 85

7.12 Values determined for the stochastic and constant terms using transverse weights and beam energy constraints. The flat weights used to constrain the transverse weights were derived using $100,120,150$, and $200 \mathrm{GeV}$ pion data and the transverse weights were derived using $200 \mathrm{GeV}$ pion data. . . 85

8.1 Properties of Standard Model Gauge Bosons. . . . . . . . . . . . . . . . 91

8.2 Properties of Standard Model fermions classified in three generations. . . . 92

9.1 Leading-Order cross-sections for the vector-boson-fusion Higgs production mass scan analysis $[60] \ldots \ldots \ldots \ldots$. . . . . . . . . . . . . 113

9.2 Cross-section and $p_{T}$ range for the QCD dijet sample. . . . . . . . . . . 114 
9.3 Particle level efficiency comparison between QCD and QCD+EW, where the efficiency ratio is defined as $(Q C D+E W) / Q C D$. See Section 10.2 for more detail on cut flow. . . . . . . . . . . . . . . . . 116

9.4 Cuts applied at the generator level. . . . . . . . . . . . . . 117

9.5 Results of the study by J. Tanaka and S. Asai on VBF Cuts bias [64]. . . . . 117

9.6 $\mathrm{W} / \mathrm{Z}+$ jet background samples divided by parton samples for the process using Alpgen/Jimmy. The "MC events" are the number of $\mathrm{MC}$ events that survive MLM matching and filtering. . . . . . . . . . . . . . . . . 118

10.1 Signal acceptance and trigger rates for the VBF invisible Higgs channel based on full ATLAS simulations. The trigger items are $\mathbb{E}_{T}(\mathrm{XE})$ central jet (J) and forward jet (FJ). The number following the menu object indicates the trigger threshold in $\mathrm{GeV}$. Both single and combined triggers are shown in this table. The values for a luminosity of $10^{33}$ are simply scaled and do not account for pile-up effects which are expected to be significant. Luminosities are in $\mathrm{cm}^{-2} \mathrm{~s}^{-1}$ units. . . . . . . . . . . . . . 136

10.2 Cross-section in fb for a $130 \mathrm{GeV}$ mass Higgs and background samples at each step of the selection process. Initial cross-section for $\mathrm{W} / \mathrm{Z}+\mathrm{jets}$ are quoted after MLM and VBF filter and NNLO correction. The first cut is the effect of the LVL1 trigger simulation with a $E_{T}$ of $100 \mathrm{GeV}$, a central jet with $p_{T}>23 \mathrm{GeV}$ and a forward jet with $p_{T}>23 \mathrm{GeV}$. Numbers in parentheses are the efficiencies for each cut . . . . . . . . . . . . . . . . . . 139

10.3 Cross-section in $\mathrm{fb}$ for each signal mass at each step in the selection cuts. The first cut is the effect of the LVL1 trigger simulation with a $\mathbb{E}_{T}$ of 100 $\mathrm{GeV}$, a central jet with $p_{T}>23 \mathrm{GeV}$ and a forward jet with $p_{T}>23 \mathrm{GeV} . \ldots 139$

10.4 Comparison between Herwig and Pythia generated samples on the selection cut for the $130 \mathrm{GeV}$ Higgs mass. Cross-section results are quoted in $\mathrm{fb}$ and cut efficiency are in parenthesis. . . . . . . . . . . . . . . . 145 
10.5 Contributions to the systematic uncertainties for signal-to-background and shape analysis. The overall systematic error was calculated by summing the errors in quadrature with the exception of the jet energy scale. In this case only the largest error from the jet energy scale was used for the overall systematic error calculation. . . . . . . . . . . . . . . . 148 


\section{List of Figures}

1.1 Schematic layout of the Large Hadron Collider showing the two counter rotating proton beams and the collision points at the four experiments. . . 3

1.2 Schematic diagram of the ATLAS detector. Note the scale drawings of people around the base of the detector. . . . . . . . . . . . 4

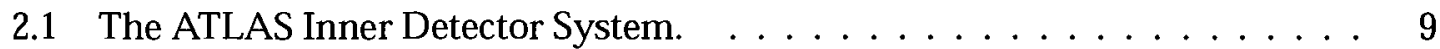

2.2 Radial profile of the central barrel layers of the ATLAS Inner Detector System. Here SCT is the Silicon Central Tracker and TRT is the Transition Radiation Tracker. . . . . . . . . . . . . . . . . . . 10

2.3 The ATLAS Calorimeter System. . . . . . . . . . . . . . . . . . . . . 12

2.4 The ATLAS Muon System. . . . . . . . . . . . . . . . . . . 15

2.5 The ATLAS Trigger/DAQ System. . . . . . . . . . . . . . . . 17

3.1 Fractional energy loss per radiation length of an electron or positron. Figure from reference $[19] \ldots \ldots \ldots \ldots \ldots$

3.2 Photon cross-section showing the contributions of different processes. The three primary mechanisms are: the photoelectric effect $\left(\sigma_{p . e .}\right)$, Compton scattering $\left(\sigma_{\text {Compton }}\right)$, and pair production via nuclear field $\left(\kappa_{n u c}\right)$. Figure from reference $[19] . \ldots \ldots \ldots \ldots \ldots \ldots \ldots \ldots$

3.3 Example of a particle shower in a sampling calorimeter. The gray and white boxes represent the absorber and active layers, respectively. . . . . . . . . 26 
4.1 The $\eta$ distribution of the two tagged jets for a $130 \mathrm{GeV}$ mass Higgs produced by VBF. The shaded area represents the FCal detector coverage. . . . . . . . 3

4.2 Schematic diagram illustrating the endcap cryostat which houses the FCal (labelled Forward detectors) modules along with the EMEC and HEC (labelled EM detector and Hadronic detector respectively.) . . . . . . . . . . . 33

4.3 Schematic diagram of the FCal modules in the ATLAS Endcap cryostat. The dashed line corresponds to the particle trajectory for $\eta=3.7 . \ldots \ldots 33$

4.4 Schematic diagrams illustrating the unique detector design of the FCal mod-

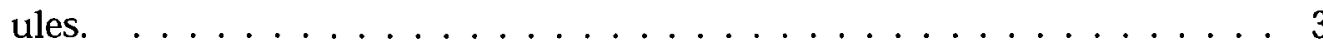

4.5 Schematic diagram of the cold electronics. Starting from the left, the electrodes are ganged together to create an electrode group. Afterwards, the groups are summed together on a transformer (summing board). Finally, the raw signal is passed to the feedthrough which connects the signal lines

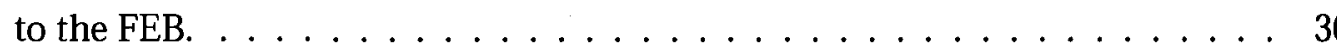

4.6 Examples of ionizing pulse shapes after electronic shaping for each of the three FCal modules. . . . . . . . . . . . . . . . . . . . 37

4.7 Schematic view of the FCal 2003 beam line. The vertical scale has been exaggerated compared to the horizontal scale for clarity. . . . . . . . . 38

4.8 Schematic view of the FCal 2003 beam spots. . . . . . . . . . . . . . . . . 39

4.9 Schematic diagram of the fully instrumented readout channels, shown in shaded areas. FCal 1 and FCal 2 emphasized the channels around the beam hole and on one side of the modules. FCal 3 was fully instrumented. . . . . 40

5.1 The average pedestal value as a function of channel number for runs between 1749 and $4315 . \ldots \ldots \ldots \ldots \ldots \ldots \ldots$

5.2 Results of noise studies illustrate instability in the electronic noise of the FCal readout channels, as shown in Figures 5.2(a) and 5.2(b). . . . . . . 46 
5.3 Profile of the noise value for every run within specified range per channel for "noisy" runs $5.3($ a) and "good" runs $5.3($ b) $\ldots \ldots \ldots \ldots \ldots \ldots$

6.1 Results of the standalone (labelled by stars) and Athena (labelled by circles) pulse shape analyses for the FCal 1 module. The error bars are for the Athena analysis and were estimated based on its maximum variation between the global pulse shape and the pulse shapes at different beam spots. The pulse shapes are in good agreement within the assumed errors. Similar comparisons were preformed for the FCal 2 and FCal 3 modules. . . . . . 56

6.2 Calibration of the OFC time for the FCal 2 module determined using 200 $\mathrm{GeV}$ pion beam data. The timing resolution was reduced by $\sim 65 \%$ within two iterations. . . . . . . . . . . . . . . . . . . . . . . 57

6.3 Results of the OFC normalized pulse shape. For all three modules the peak amplitude is within $1 \%$ of unity. $\ldots \ldots \ldots \ldots \ldots$

7.1 Example of S1 and S2 combination information. It is clear that cutting on S1 or S2 individually would remove potentially valid events. Therefore, a cut on the combined signal of S1 and S2 is used to remove events that fall in the shaded area . . . . . . . . . . . . . . . . . . . . . . . 61

7.2 Plots of $E_{1, \max } / E_{1}$ relative to $\mathrm{x}$ and $\mathrm{y}$ using the shift corrections for the

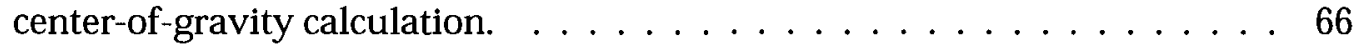

7.3 Plots of $E_{1, \max } / E_{1}$ relative to $\mathrm{x}$ and $\mathrm{y}$ using the refined shift corrections. . . 66

7.4 Energy distribution for electrons in the $4 \mathrm{~L}$ position using an $8 \mathrm{~cm}$ cylinder cluster. The bullets are electron data, the solid line is the 7 parameter fit, and the dashed line represents the pion fit. Note that the $20 \mathrm{GeV}$ pion data was not available for the background fit. . . . . . . . . . . 71

7.5 Electron energy linearity plot 7.5(a) with residual of the energy linearity fit 7.5 (b) using various cylinder cluster sizes. . . . . . . . . . . . 73 
7.6 Electron energy resolution results are shown in Figure 7.6(a) with noise subtracted in quadrature using noise values shown in Figure 7.6(b) using various cylinder cluster sizes. . . . . . . . . . . . . . . . 76

7.7 Energy distribution for pions in the $4 \mathrm{~L}$ position using a $16 \mathrm{~cm}$ cylinder cluster and transverse weights. The points are pion data, the solid line is the 6 parameter fit. ....................

7.8 Comparison of the energy response using flat weights derived from a 200 $\mathrm{GeV}$ pion beam data with and without beam constraints as a function of beam energy. The ratio between the reconstructed energy and the beam energy, $E_{\text {Reco }} / E_{\text {Beam }}$, was used to quantify the beam response. . . . . . . .

7.9 Transverse weights for all three FCal modules derived from 120, 150, and $200 \mathrm{GeV}$ energy hadrons. . . . . . . . . . . . . . . . . . . 84

7.10 Comparison of the energy response using transverse weights derived with and without beam constraints. The flat weights used to constrain the transverse weights were derived from $200 \mathrm{GeV}$ pion data. The ratio between the reconstructed energy and the beam energy, $E_{\text {Reco }} / E_{B e a m}$, was used to quantify the beam response. $\ldots \ldots \ldots \ldots \ldots \ldots$

7.11 Comparison of the energy response between beam constrained flat and transverse weights. The ratio between the reconstructed energy and the beam energy, $E_{R e c o} / E_{\text {Beam }}$, was used to quantify the beam response. . . . .

7.12 Comparison of the energy resolution between beam constrained flat and transverse weights. . . . . . . . . . . . . . . .

8.1 Electroweak precision data as a function of Higgs mass [33]. The solid line is the result from the fit and the blue band is the theoretical uncertainty for missing higher order corrections. The yellow band is the excluded region from direct searches at LEP. Here $\Delta \chi^{2}=\chi^{2}-\chi_{\text {min }}^{2} \ldots \ldots \ldots \ldots$. . . . . 95 
8.2 Constraints on the Higgs mass as a function of the effective energy scale [34]. The upper bound is the constraint provided by the triviality condition. The lower bound is provided by the vacuum stability condition, that is, the breaking of electroweak symmetry. In addition, constraints from direct searches and electroweak precision measurements are shown. The white band indicates the favored Higgs mass range between approximately 115-

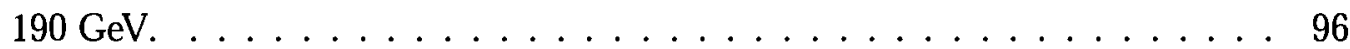

8.3 Higgs production cross-section in the Standard Model [35]. . . . . . . . . 98

8.4 Feynman diagrams for the Higgs production modes. . . . . . . . . . . . . 99

8.5 The Standard Model Higgs branching ratio as a function of Higgs mass [19]. 99

8.6 Invisible Higgs branching ratio, $\Gamma\left(h \rightarrow \nu_{4} \nu_{4}\right)$, as a function of Higgs mass assuming $m_{\nu_{4}}=50 \mathrm{GeV}$ [43] for a model that includes massive $4^{\text {th }}$ generation neutrinos. The line associated with the label NN is the $h \rightarrow \nu_{4} \nu_{4}$ branching ratio. . . . . . . . . . . . . . . . . . . 101

8.7 Invisible Higgs branching ratio, $\Gamma(h \rightarrow \mathcal{G})$, as a function of $\xi$ with two extra compactified dimensions for various $M_{D}$ energy scales [47] for a $120 \mathrm{GeV}$ Higgs mass. . . . . . . . . . . . . . . . . . . . . . . . . . . 102

8.8 The $95 \%$ confidence level sensitivity limit, $\xi^{2}$, as a function of Higgs mass for invisible Higgs boson decays in the $\mathrm{ZH}, t \bar{t} \mathrm{H}$ and VBF production assuming an integrated luminosity of $30 \mathrm{fb}^{-1}$ using the ATLAS detector [50]. These analyses used fast simulation data. . . . . . . . . . . . . . 105

9.1 Schematic diagram of the ATLAS production sequence. The rectangular boxes represent the production stages and the oval boxes represent the output data . . . . . . . . . . . . . . . . . . 107 
9.2 Illustration of the MLM prescription. The solid line represents the partons from the matrix elements calculation, the dashed line represents the partons produced during the showering stage, and the cones represent the jets constructed using all the partons after showering. The length of the parton lines is used as an indication of their $p_{T}$, as can be seen in Figure 9.2(d). . . . 112

9.3 Examples of the hard scattering processes. . . . . . . . . . . 115

9.4 Comparison between calorimeter energy resolution (solid line) and tracker momentum resolution (dashed line) as function of $p_{T} \ldots \ldots \ldots 123$

10.1 Cross-section in arbitrary units for the signal and background as a function of the $p_{T}$ of the tagged jets. Signal events are for a $130 \mathrm{GeV}$ mass shown in bullets and background samples Z+jets, W+jets, and QCD dijets are shown in open crosses, triangles, and diamonds, respectively. The distributions are normalized to unity. . . . . . . . . . . . . . . . . . 130

10.2 Cross-section in arbitrary units for the signal and background as a function of tagged jet $\eta$ dependent variables with no cut on the $p_{T}$. Signal events are for a $130 \mathrm{GeV}$ mass shown in bullets and background samples Z+jets, W+jets, and QCD dijets are shown in open crosses, triangles, and diamonds, respectively. The distributions are normalized to unity. . . . . . 131

10.3 Cross-section in arbitrary units for the signal and background as a function of the invariant mass of the tagged jets. Signal events are for a $130 \mathrm{GeV}$ mass shown in bullets and background samples Z+jets, W+jets, and QCD dijets are shown in open crosses, triangles, and diamonds respectively. The distributions are normalized to unity. . . . . . . . . . . . . 131 
10.4 Cross-section in arbitrary units for the signal and background as a function of $\mathbb{E}_{T}$. Signal events are for a $130 \mathrm{GeV}$ mass shown in bullets and background samples Z+jets, W+jets, and QCD dijets are shown in open crosses, triangles, and diamonds respectively. The distributions are normalized to unity. . . . . . . . . . . . . . . . . . . . . . 132

10.5 Cross-section in arbitrary units for the signal and background as a function of $I=\min \left[\phi\left(\mathscr{F}_{T}\right)-\phi\left(j_{1,2}\right)\right]$. Signal events are for a $130 \mathrm{GeV}$ mass shown in bullets and background samples Z+jets, $\mathrm{W}+\mathrm{jets}$, and QCD dijets are shown in open crosses, triangles, and diamonds respectively. The distributions are normalized to unity. . . . . . . . . . . . . . . . . 13

10.6 Cross-section in arbitrary units for the signal and background as a function of the azimuthal angle between the tagged jets, $\phi_{j j}$. Signal events are for a $130 \mathrm{GeV}$ mass shown in bullets and background samples $Z+j e t s, W+j e t s$, and QCD dijets are shown in open crosses, triangles, and diamonds respectively. The distributions are normalized to unity. . . . . . . . . . . . . . . . 134

10.7 Sensitivity limit using the signal-to-background analysis for a $99 \%$ confidence level using an integrated luminosity of $30 \mathrm{fb}^{-1}$. All the cuts from Table 10.2 have been applied to the signal and background samples. Results with and without systematic errors are shown both for this analysis and an earlier analysis that used fast simulation events. In addition, the Monte-Carlo statistics error was investigated and found to be negligible. . 141 
10.8 Discovery potential for an invisible Higgs at $99 \%$ confidence level via the VBF channel using shape analysis for an integrated luminosity of $30 \mathrm{fb}^{-1}$ with and without systematic errors. The black triangles and circles are the results from this analysis with and without systematic errors respectively. The open triangles are results from O. J. P.Eboli and D. Zeppenfeld [78] using data produced at the event generator level. The results from $\mathrm{O}$. J. P.Eboli and D. Zeppenfeld analysis were quoted for $95 \%$ confidence level, therefore a scale factor was applied to produce the $99 \%$ confidence level results presented in this figure. In addition, the Monte-Carlo statistics error was investigated and found to be negligible. . . . . . . . . . . . . . . 144 


\section{Chapter 1}

\section{Introduction}

The year 2008 promises to be a very exciting one for particle physicists and the reason for this excitement is the turn on of the Large Hadron Collider (LHC). The LHC will provide proton-proton collisions at energies that far exceed the energy of any accelerator in the history of humankind. These collisions will reproduce conditions similar to those that existed less than a billionth of a second after the big bang. By creating these conditions, physicists can improve their understanding of the basic constituents of the Universe and the forces that act between them. Preparations are well advanced for the detectors that will examine the collisions that will be produced by the LHC, with work continuing on their commissioning and calibration and simulation of physics processes that will be studied at the LHC. Presented in this thesis, are studies related to the calibration of one of the detectors and an analysis of a new physics process that could be observed at the LHC. Before discussing these studies, a survey of our present understanding of the particles that make up the Universe and the forces that act between them is in order.

In our current model of the Universe, there are four forces which govern all interactions: gravity, electromagnetism, the weak and the strong nuclear forces. There are presently two theories which describe these forces. The theory of General Relativity is our model of the gravitational force and provides a description of large scale physics, such as the for- 
mation of stars and galaxies. The Standard Model (SM), on the other hand, provides an explanation for the subatomic interactions governed by the electromagnetic, weak, and strong forces. The collisions at the LHC are intended to probe our understanding of the SM. Within the SM, there are two types of fundamental particles: spin 1/2 fermions and integer spin bosons. Fermions are the particles which constitute matter and are classified as either quarks or leptons. In contrast, bosons are particles which mediate the interactions between the fermions. Presently, all experimental results agree remarkably well with the predictions of the SM. However, there is one element that has not been experimentally confirmed in the SM and that is related to the way in which all the fundamental particles acquire mass. In the SM, the way to generate massive particles is via the Higgs mechanism which requires the existence of a new particle named the Higgs boson. However, the Higgs particle, which was originally proposed in the 1960's, has yet to be discovered. It should be noted that the Higgs mechanism is not the only possible explanation and other theories have been proposed. These theories could lead to new physics that might also be accessible at the LHC. One possible outcome of these theories is a Higgs particle which decays into particles which do not produce a signal in the experiment and therefore requires special analysis techniques for its detection. The central goal of the LHC is to create collisions with sufficient energy to discover the SM Higgs particle and to search for new phenomena.

The LHC will accelerate two counter rotating proton beams to produce a high energy collision with a center-of-mass energy of $14 \mathrm{TeV}$. Given that both beams are composed of the same particle type, two separate beam-lines are required. The LHC machine components have been installed at CERN in a $27 \mathrm{~km}$ circumference tunnel which is 100 meters below the Earth's surface. The LHC machine consists of an array of magnets to bend, steer, and focus the beams. In addition to providing the highest energy collisions, the LHC will also provide the highest collision rate. The first phase of data taking will be at a luminosity of $10^{33} \mathrm{~cm}^{-2} \mathrm{~s}^{-1}$ and should provide an integrated luminosity of 10 inverse femtobarn $\left(\mathrm{fb}^{-1}\right)$. 
Afterwards, the luminosity will increase to the design luminosity of $10^{34} \mathrm{~cm}^{-2} \mathrm{~s}^{-1}$ resulting in $100 \mathrm{fb}^{-1}$ of data per year. The combination of high beam energies and collision rates at the LHC opens up a new energy regime and provides access to exciting new physics.

In order to measure the collisions produced by the LHC, four detectors are being developed. These detectors must be very well understood and properly calibrated in order to provide reliable information. There are four interaction points ${ }^{1}$ at the LHC which are associated with each experiment, as illustrated in Figure 1.1. There are two general purpose

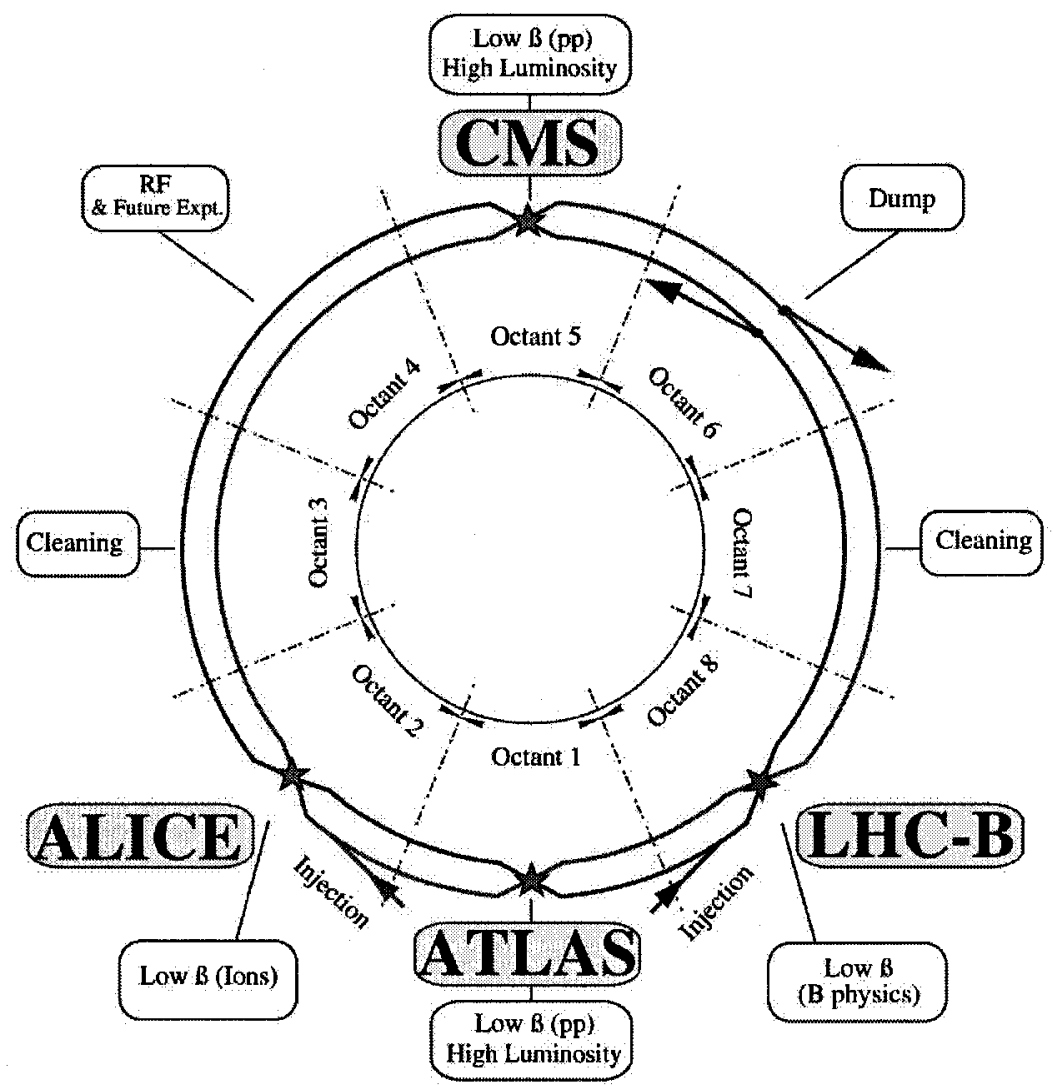

Figure 1.1: Schematic layout of the Large Hadron Collider showing the two counter rotating proton beams and the collision points at the four experiments.

detectors, A Toroidal LHC Apparatus (ATLAS) and the Compact Muon Solenoid (CMS), which are designed to measure leptons, jets, and missing transverse energy. These two

\footnotetext{
${ }^{1}$ Location in which the counter rotating beams intersect (collide).
} 
experiments are placed at opposing points on the ring. The enormous size of the ATLAS detector, as shown in Figure 1.2, can be inferred by the size of the people shown schematically at the base of the detector. There are two additional LHC intersection regions which

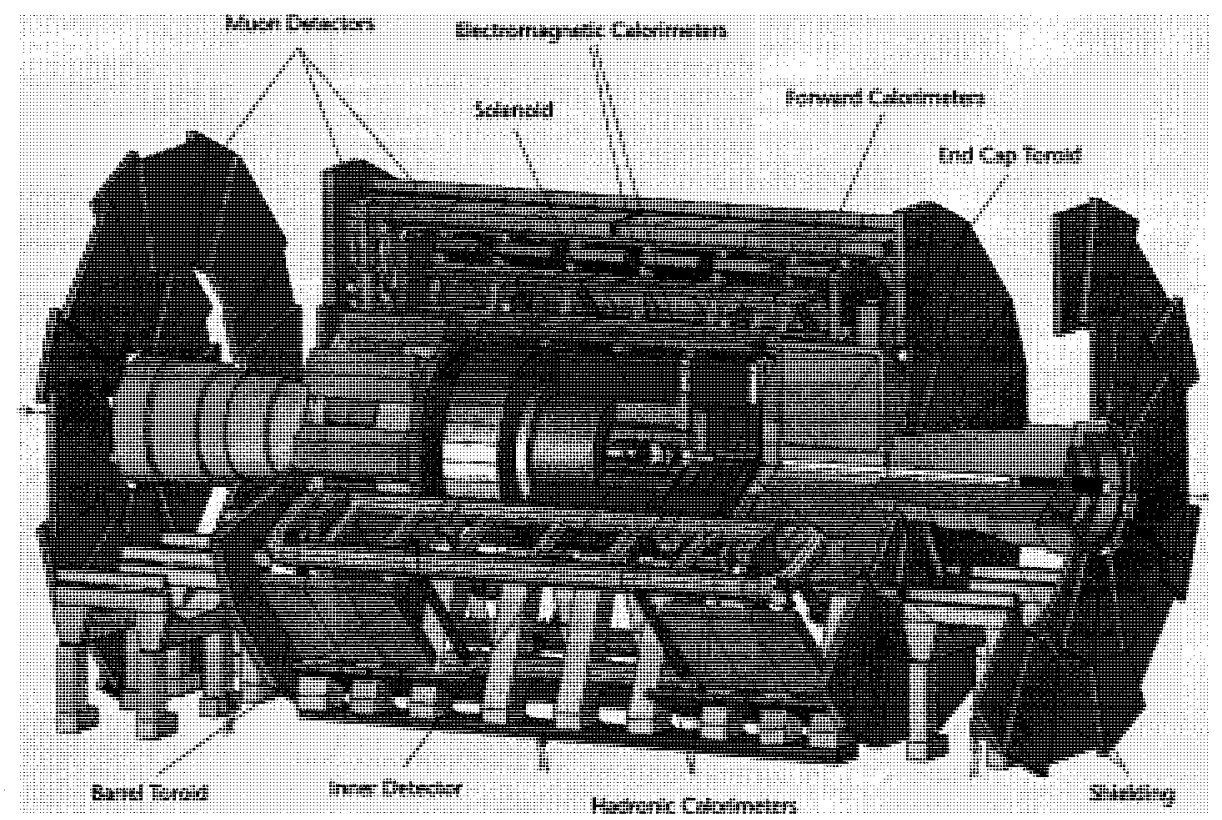

Figure 1.2: Schematic diagram of the ATLAS detector. Note the scale drawings of people around the base of the detector.

house the ALICE (A Large Ion Colliding Experiment) and LHCb experiments. In contrast to ATLAS and CMS, the ALICE experiment is a dedicated heavy-ion device which is optimized to study heavy ion collisions provided by the LHC, while LHCb will specialize in bottom quark physics.

The work in this thesis is related to the calibration and exploitation of the ATLAS experiment. Chapter 2 provides a brief survey of the component detectors of ATLAS. Afterwards, a review of the principles of calorimetry, as applied to high-energy physics, is presented in Chapter 3. The main body of the thesis is divided into two parts: the performance of the ATLAS Forward Calorimeter and the discovery potential for the invisible decay of the Higgs particle via the vector-boson-fusion mode. 
In the first part of this thesis, an analysis of the 2003 test beam of the ATLAS Forward Calorimeter is used to extract the FCal performance characteristics. Chapter 4 summarizes the goals and setup of the test beam. Chapters 5 and 6 discuss how the data was processed in order to reconstruct the energy in the calorimeters. Finally the measured performance of the ATLAS FCal detector for both electrons and pions is discussed in Chapter 7. These performance measurements will provide the initial calibration for the FCal detectors.

The second part of this thesis focuses on the discovery potential of the invisible Higgs via the vector-boson-fusion mode using the ATLAS detector. A brief review of the Standard Model of particle physics and the motivation for the invisible Higgs study is presented in Chapter 8. Afterwards, a summary of the Monte-Carlo data production and the reconstruction tools is provided in Chapter 9. The data analysis, including limits on the discovery potential for the invisible Higgs is presented in Chapter 10. 


\section{Chapter 2}

\section{LHC and the ATLAS Experiment}

\subsection{The LHC}

The Large Hadronic Collider (LHC) is a proton-proton and heavy-ion collider located at CERN. The accelerator is close to completion and will be commissioned in the summer of 2008. When the LHC turns on, it will be the world's largest proton-proton and heavy-ion collider. In the proton-proton operating mode, the center-of-mass energy will be $14 \mathrm{TeV}$, superseding the Tevatron which presently has the largest center-of-mass energy at 1.96 TeV. In addition, when the LHC reaches its design luminosity, it will provide $\sim 100 \mathrm{fb}^{-1}$ of integrated luminosity per year, surpassing all previous hadron colliders.

In addition to proton-proton collisions, the LHC will also collide heavy ions, which will be the focus for the ALICE experiment. The LHC has a large discovery potential from low energy B meson physics to exotic new physics at the TeV scale.

There are four stages required to inject protons into the LHC. First, the protons are produced in the $50 \mathrm{MeV}$ proton linear accelerator, then injected into the $1.4 \mathrm{GeV}$ Proton Synchrotron Booster. Afterwards, they are accelerated from $1.4 \mathrm{GeV}$ to $26 \mathrm{GeV}$ using the Proton Synchrotron (PS). Finally, the Super Proton Synchrotron (SPS) accelerates the protons 
to $450 \mathrm{GeV}$ before final injection into the LHC ring.

Once the beam is injected into the LHC, it is accelerated to the design energy using superconducting radio frequency cavities. In order to keep the beam circulating within the trajectory of the LHC tunnels, an 8.4 Tesla magnetic field is required. This magnetic field is achieved by using 1232 superconducting dipole magnets. The beam is steered and focused using multipole magnets. In all, a total of 9300 magnets are used to optimize the particle trajectories. The beam is composed of discrete bunches of protons spaced every $25 \mathrm{~ns}$, each bunch containing $10^{11}$ protons at high luminosity. The LHC beams are focused to produce collisions at four different Interaction Points (IP), one of which is located at the center of the ATLAS experiment.

\subsection{The ATLAS Experiment}

The ATLAS detector is an extremely complex general purpose detector designed to measure the properties of the remnant particles created by the LHC proton-proton collisions. Its enormous size is quite remarkable given its dimensions of $25 \mathrm{~m}$ in diameter, and 46 $\mathrm{m}$ in length and its weight of 7,000 tonnes. In addition, the ATLAS detector has approximately 100 million readout channels and $3,000 \mathrm{~km}$ of cables are required for interconnections, power and monitoring. However, what is most impressive about the ATLAS detector is the precision to which it can measure particle properties such as position, momentum and mass. It is the combination of the properties of each individual sub-detector component which allows for these precise measurements. This chapter provides a brief summary of the characteristics of the ATLAS detector. A summary of the overall performance goals can be found in Table 2.1 and are described in this chapter. A complete description of the ATLAS detectors can be found in the Technical Design Reports [1-14]. 


\begin{tabular}{lcc}
\hline Sub-Detector & Resolution Goal & $\eta$ Coverage \\
\hline \hline Inner detectors & $0.05 \% p_{T} \oplus 1 \%$ & \pm 2.5 \\
\hline EM Calorimeters & $10 \% / \sqrt{E} \oplus 1 \%$ & \pm 3.2 \\
\hline Hadronic Calorimeters (jets) & & \\
$\quad$ Barrel and End-cap & $50 \% / \sqrt{E} \oplus 3 \%$ & \pm 3 \\
$\quad$ Forward Calorimeters & $100 \% / \sqrt{E} \oplus 10 \%$ & $3.1<|\eta|<4.9$ \\
\hline Muon detector & $10 \%$ at $p_{T}=1 \mathrm{TeV}$ & \pm 3 \\
\hline \hline
\end{tabular}

Table 2.1: Summary of the ATLAS detector performance goals. The units for energy, E, and transverse momentum, $p_{T}$, are in $\mathrm{GeV}$ in the particle physics system, where $c$ and $\hbar$ are set to 1 . The variable, $\eta$, is defined in Section 2.3.

\subsection{The ATLAS Coordinate System}

The LHC beam line is defined to be along the z-axis and the transverse plane is defined by the $x-y$ plane. The positive $x$-axis points towards the center of the LHC ring, while the positive $y$-axis is pointing upwards. Two angles are used to describe the system, $\theta$ and $\phi$ which are the polar and azimuthal angles, respectively. The pseudo-rapidity is commonly used to describe the angle of a particle relative to the beam axis and is defined as $\eta \equiv-$ $\ln \tan (\theta / 2)$. This variable is preferred over the polar angle, $\theta$, since particle production is approximately constant as a function of $\eta$. In addition, the separation between particles in $\eta$ is independent of Lorentz boosts along the beam axis.

Properties such as the transverse momentum, $p_{T}$, are defined by the sum in quadrature of the $\mathrm{x}$ and $\mathrm{y}$ contributions. Transverse measurements are often used since there is large uncertainty in the initial momentum of hard collisions along the $z$ direction. This is because protons are composite particles and the collisions of interest are between the proton constituents, the quarks and gluons, which carry a fractional amount of the proton's momentum. 


\subsection{The ATLAS Inner Detector}

The ATLAS inner detector system is the closest to the IP. The inner detector system is composed of three sub-detectors: the pixels, the Silicon Central Tracker (SCT) and the Transition Radiation Tracker (TRT) as shown in Figures 2.1 and 2.2. The combination

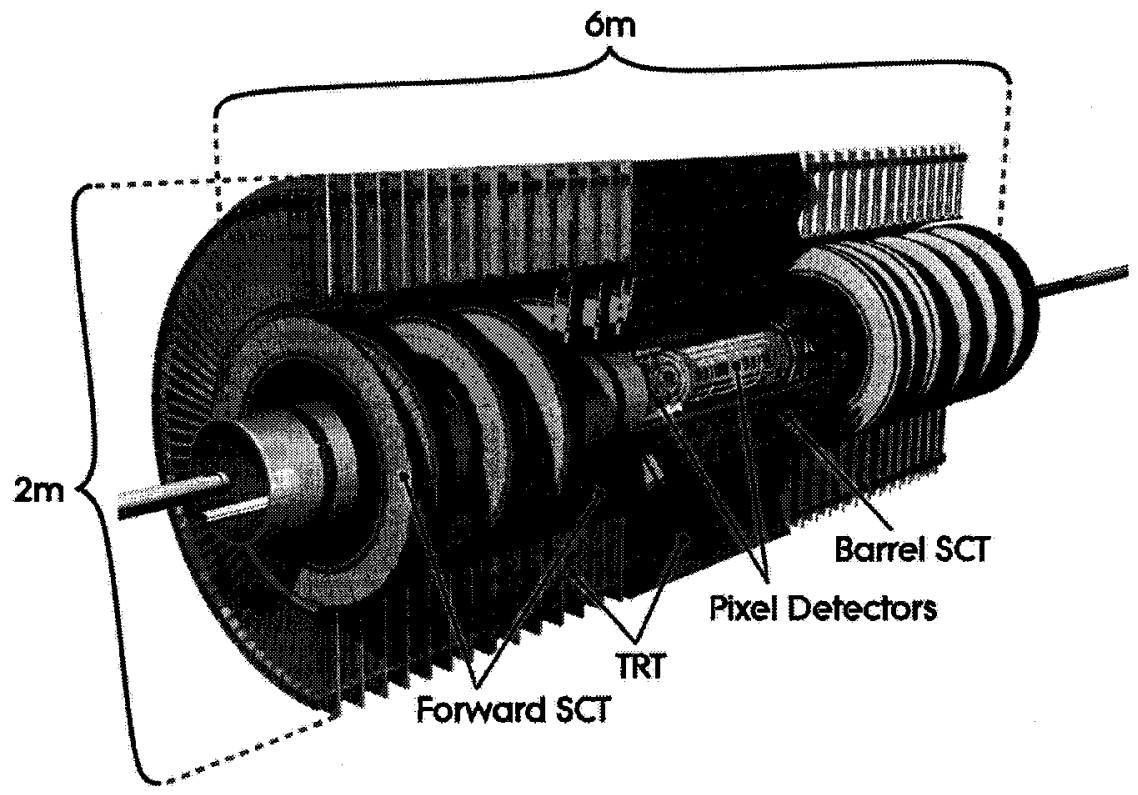

Figure 2.1: The ATLAS Inner Detector System.

of these three sub-detectors provides a coverage of $|\eta| \leqslant 2.5$, measures charged particle tracks, momentum, vertexes, and provides particle identification. This system is expected to provide a resolution in $p_{T}$ that is better than $0.05 \% p_{T} \oplus 1 \%$ within $\eta<2.5$, where $p_{T}$ is in $\mathrm{GeV}$. The following is a summary of the characteristics for each sub-detector and the central solenoid magnet.

The Pixel Detectors (PD) are the inner-most detectors and must be able to survive the large flux of particles at full luminosity. These detectors are designed to provide excellent pattern recognition, impact parameter resolution, vertexing, and $\mathrm{b}$ meson particle identification and triggering. The pixel detectors are arranged into three 


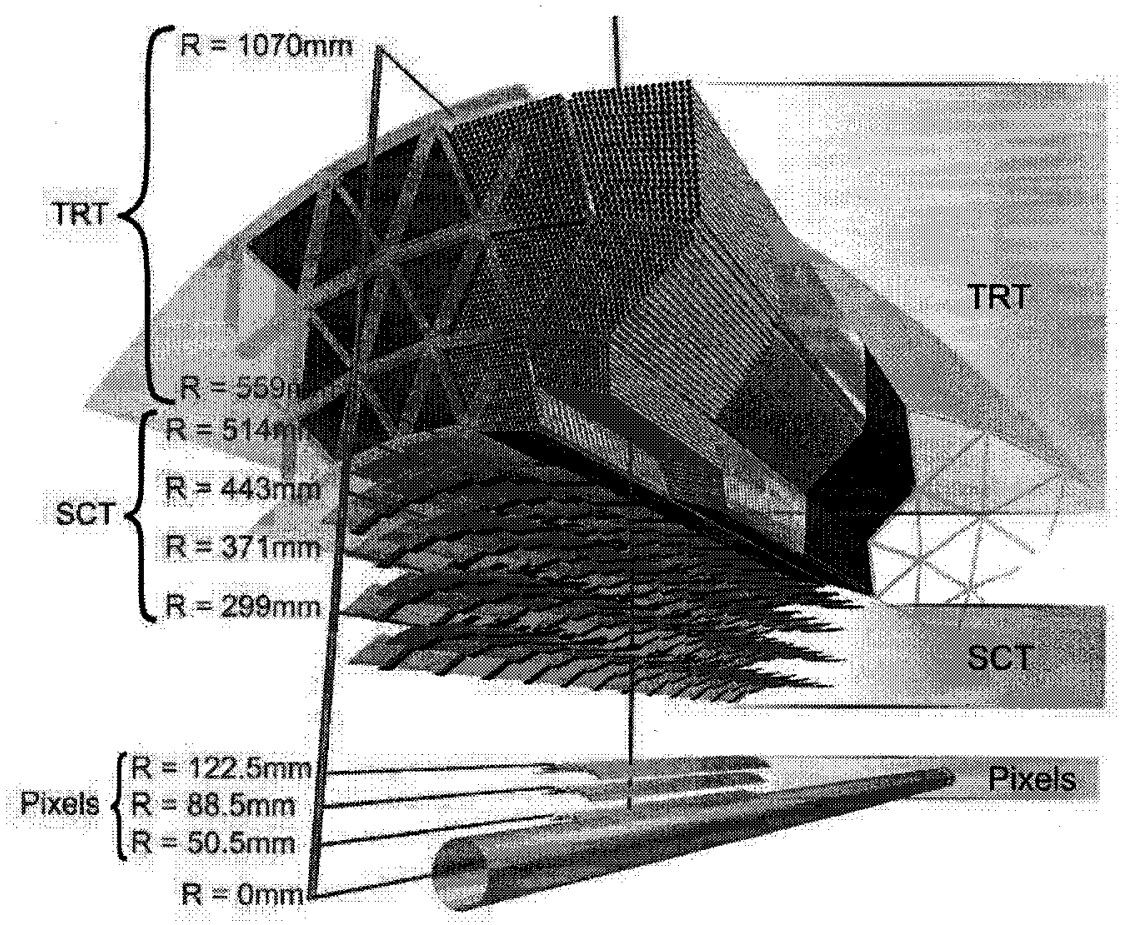

Figure 2.2: Radial profile of the central barrel layers of the ATLAS Inner Detector System. Here SCT is the Silicon Central Tracker and TRT is the Transition Radiation Tracker.

barrel layers that surround the IP and ten disk layers to measure forward tracks, five on each side of the IP. There are 140 million detector elements providing high granularity with a position resolution of $\sim 12 \mu \mathrm{m}$ and $\sim 70 \mu \mathrm{m}$ in the $R \phi$ and $\mathrm{z}$ direction, respectively.

The Silicon Microstrip detectors surround the pixel detectors with four central barrel layers and nine wheels in the forward and backward directions of the IP. These detectors contribute to pattern recognition, impact parameter resolution, and vertex positioning. They are designed to provide eight measurements per track with a spatial resolution of $\sim 16 \mu \mathrm{m}$ in $R \phi$ and $\sim 580 \mu \mathrm{m}$ in the $\mathrm{z}$ direction.

The Transition Radiation Tracker (TRT) is the furthest inner detector from the IP and uses "straw tube" detectors. Each straw tube has a diameter of approximately 4 $\mathrm{mm}$. The TRT detectors contribute to pattern recognition and electron identification. Electron identification is achieved by looking at the number of transition radi- 
ation photons. Electrons are expected to produce a larger number of photons due to transition radiation than pions or other charged particles. The TRT is designed to provide 36 measurements and a spatial resolution of $\sim 170 \mu \mathrm{m}$ per hit.

The Central Solenoid Magnet surrounds the inner detector system and provides a 2 Tesla magnetic field in the region of the inner detector for the momentum measurement of tracks. This superconducting magnet measures $5.3 \mathrm{~m}$ in length, $2.4 \mathrm{~m}$ in diameter, and is $4.5 \mathrm{~cm}$ thick. The solenoid magnet is located between the IP and the electromagnetic calorimeter and as such degrades the performance of the electromagnetic calorimeter by introducing additional material. To minimize this effect, the solenoid is integrated into the Liquid Argon (LAr) cryostat, removing the need for additional vacuum walls (i.e. more material).

\subsection{The ATLAS Calorimeters}

The ATLAS calorimeter system, as shown Figure 2.3, is designed to measure the position and energy of electrons, positrons, photons, and jets, as well as to provide a measurement for the missing transverse energy. The ATLAS calorimeter system is hermetic providing continuous coverage within $|\eta|<5$. Hermetic calorimetry greatly improves the determination of event variables such as missing transverse energy, $\mathbb{H}_{T}$. This permits the investigation of exotic discovery channels many of which result in particles that are not observed in the detector leading to missing energy in events. An example of this is a non-standard model Higgs which decays invisibly. There are four detector technologies implemented within the ATLAS calorimeter system. These technologies were chosen to satisfy the physics requirements and at the same time meet the challenges of the LHC environment. The ATLAS calorimeter system is composed of calorimeters that are optimized either for electromagnetic or hadronic shower measurements. Radiation hard liquid argon devices are used for the electromagnetic calorimeter and for the hadronic calorimeters in the forward regions where particle fluxes are highest. There are three 


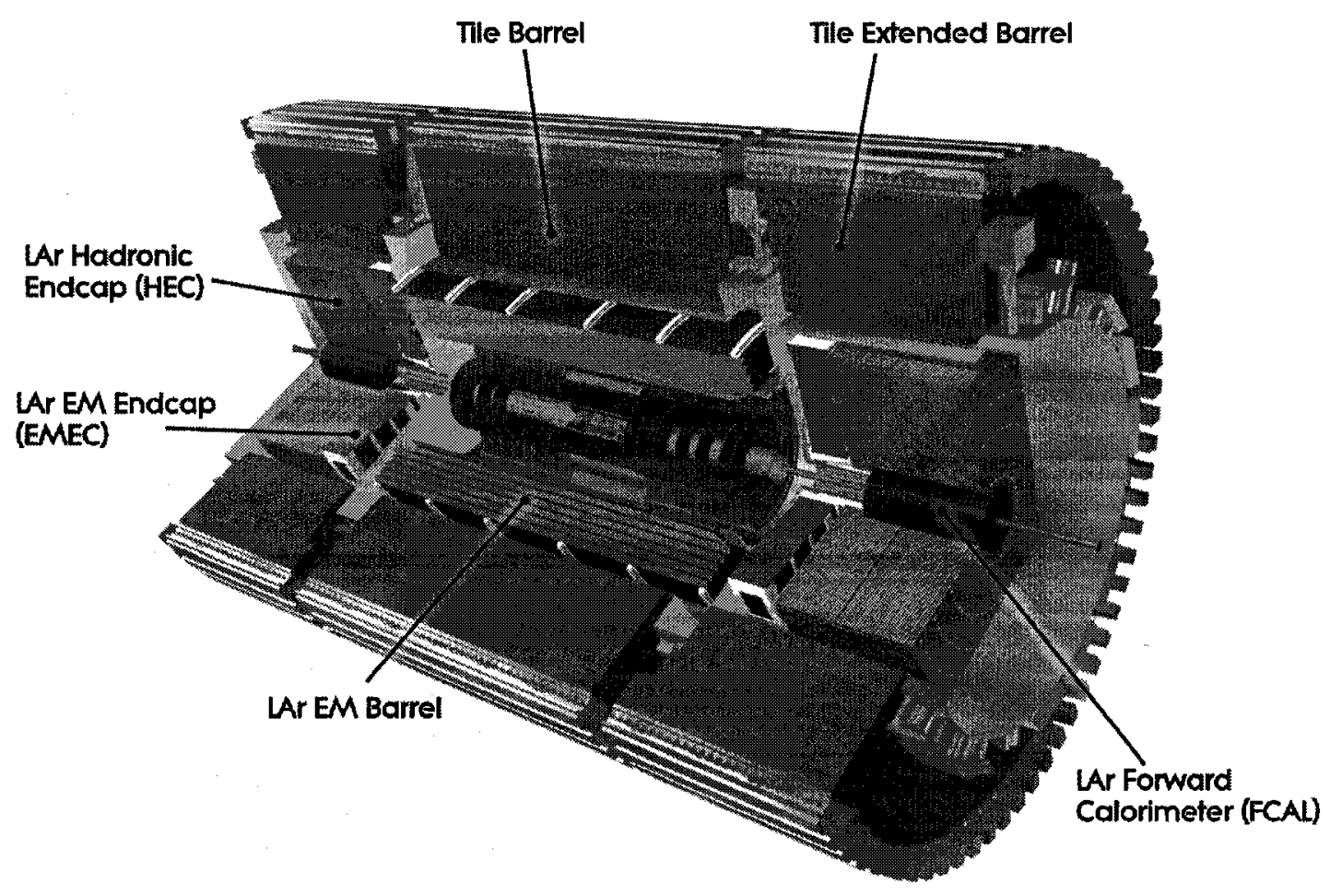

Figure 2.3: The ATLAS Calorimeter System.

cryostats which house the liquid argon detectors, one barrel and two endcap cryostats (one for each side of the IP). In addition, the hadronic calorimetry in the barrel is composed of iron plus tile scintillator detectors. The following briefly describes the detectors which constitute the calorimeter system.

The Electromagnetic Calorimeter consists of three parts, one barrel $(|\eta|<1.475)$ and two end-caps $(1.375<|\eta|<3.2)$. They are made of lead and liquid argon and provide a coverage of $|\eta|<3.2$. The electrodes for the barrel have an accordion shape which provides $\phi$ symmetry without any cracks. Some of the main requirements for this detector come from the $H \rightarrow \gamma \gamma$ and $H \rightarrow Z Z \rightarrow 4 e$ decay modes. These decay modes require a large $\eta$ coverage, fine segmentation, a large energy measurement range, and good energy resolution. The design requirement for the EM calorimeter energy resolution is a stochastic term less than $10 \% / \sqrt{E}$ and a constant term less than $1 \%$, where $E$ is in $\mathrm{GeV}$ (see Section 3.2.2 for a summary of the energy resolution 
parameterization). Results from test beams for beam energies between 10 and 245 $\mathrm{GeV}$ give a stochastic term below $10 \% / \sqrt{E}$ for the barrel and $12.5 \% / \sqrt{E}$ for the endcap and a constant term below $0.3 \%[15,16]$, where $E$ is in $\mathrm{GeV}$. These results show that the EM calorimeter has achieved its key design performance goals. In addition, a presampler detector is used for $|\eta|<1.8$ to correct for the energy lost by electrons and photons upstream of the calorimeter. This presampler consists of a $1.1 \mathrm{~cm}(0.5$ $\mathrm{cm})$ thick LAr layer in the barrel and end-cap regions.

The Hadronic Barrel Calorimeter (TileCal) is composed of one central barrel and two extended barrels, providing a coverage of $|\eta|<1.7$. These barrels are made of 3 $\mathrm{mm}$ thick scintillator tiles placed between $14 \mathrm{~mm}$ thick iron absorber plates and are read out using wavelength shifting fibers and photo-multiplier tubes. The main requirement of this detector is to provide jet energy measurements and to assist in determining the $\mathbb{E}_{T}$. The ATLAS energy resolution requirement for the hadronic calorimeter system within $|\eta|<3$ is a stochastic term of $50 \% / \sqrt{E}$ and a constant term of less than $3 \%$, where $E$ is in $\mathrm{GeV}$. Test beam results for hadrons hitting the calorimeter at an angle equivalent to $\eta=0.25$, show a stochastic term of $(57.5 \pm$ $0.5) \% / \sqrt{E}$ and a constant term of $5.9 \pm 0.1 \%$, while for $\eta=0.55$ they show a stochastic term of $(53.1 \pm 0.4) \% / \sqrt{E}$ and a constant term of $5.2 \pm 0.1 \%$, where $E$ is in $\mathrm{GeV}$. These results show that the detector resolution displays a small yet significant $\eta$ dependence.

The Hadronic End-Cap Calorimeters (HEC) are made of copper plates with liquid argon as the active medium and provide a coverage of $1.5<|\eta|<3.2$. The use of liquid argon was motivated by its ability to withstand the harsh radiation environment that will be experienced by the HEC. As with the TileCal, the HEC is used in reconstructing jet energies and contributes to the $\mathbb{F}_{T}$. The energy resolution requirement is a stochastic term of $50 \% / \sqrt{E}$ and a constant term less than $3 \%$, where $E$ is in $\mathrm{GeV}$. From pion test beam results using the EM End-Cap and HEC modules the en- 
ergy resolution was parameterized with a stochastic term of $(81.7 \pm 0.3) \% / \sqrt{E}$ and of $(84.6 \pm 0.3) \% / \sqrt{E}$ for $\pi^{-}$and $\pi^{+}$and a constant term of zero within errors [17], where $E$ is in $\mathrm{GeV}$.

The Forward Calorimeters (FCal) consist of three longitudinal segments, one electromagnetic copper-liquid argon section and two hadronic tungsten-liquid argon sections which provide a coverage of $3.1<|\eta|<4.9$. The additional $\eta$ coverage provided by the FCal improves the overall acceptance and $\mathscr{E}_{T}$ determination. This is key for searches such as the invisible Higgs via vector-boson-fusion which requires both good forward jet tagging and $\mathbb{E}_{T}$. The designed energy resolution for the FCal is a stochastic term of $100 \% / \sqrt{E}$ and a constant term less than $10 \%$, where $E$ is in $\mathrm{GeV}$. These requirements have been achieved and will be presented in Chapter 7 . It should be noted that the tracking system only extends to $|\eta|<2.5$ and therefore no tracking information is available for particles or jets hitting the forward calorimeter. Hence, for this region, calibrations obtained from test beams are particularly important.

\subsection{The ATLAS Muon System}

The ATLAS muon system, as shown in Figure 2.4, provides a stand-alone measurement of the momenta of muons and also has a stand-alone trigger. The physics requirements are dominated by the requirements for a search for the Higgs boson via $H \rightarrow Z Z \rightarrow 4 \mu$ and exotic physics searches such as $Z^{\prime} \rightarrow \mu \mu$, and $W^{\prime} \rightarrow \mu \nu$. The requirements of this system include a mass resolution of $\sim 1 \%$, a spatial resolution of 5-10 $\mathrm{mm}$ to enable reliable track and momentum reconstruction, and high acceptance in both precision measurements and the trigger sub-system. In addition, the muon system provides a transverse momentum resolution of $10 \%$ at $p_{T}=1 \mathrm{TeV}$ and is one of the sub-systems used in determining $\mathbb{E}_{T}$.

The Muon Magnet System consists of three toroid magnets providing a magnetic field covering an $\eta$ range of $|\eta|<2.7$. The Barrel Toroid (BT) is composed of 8 barrel 


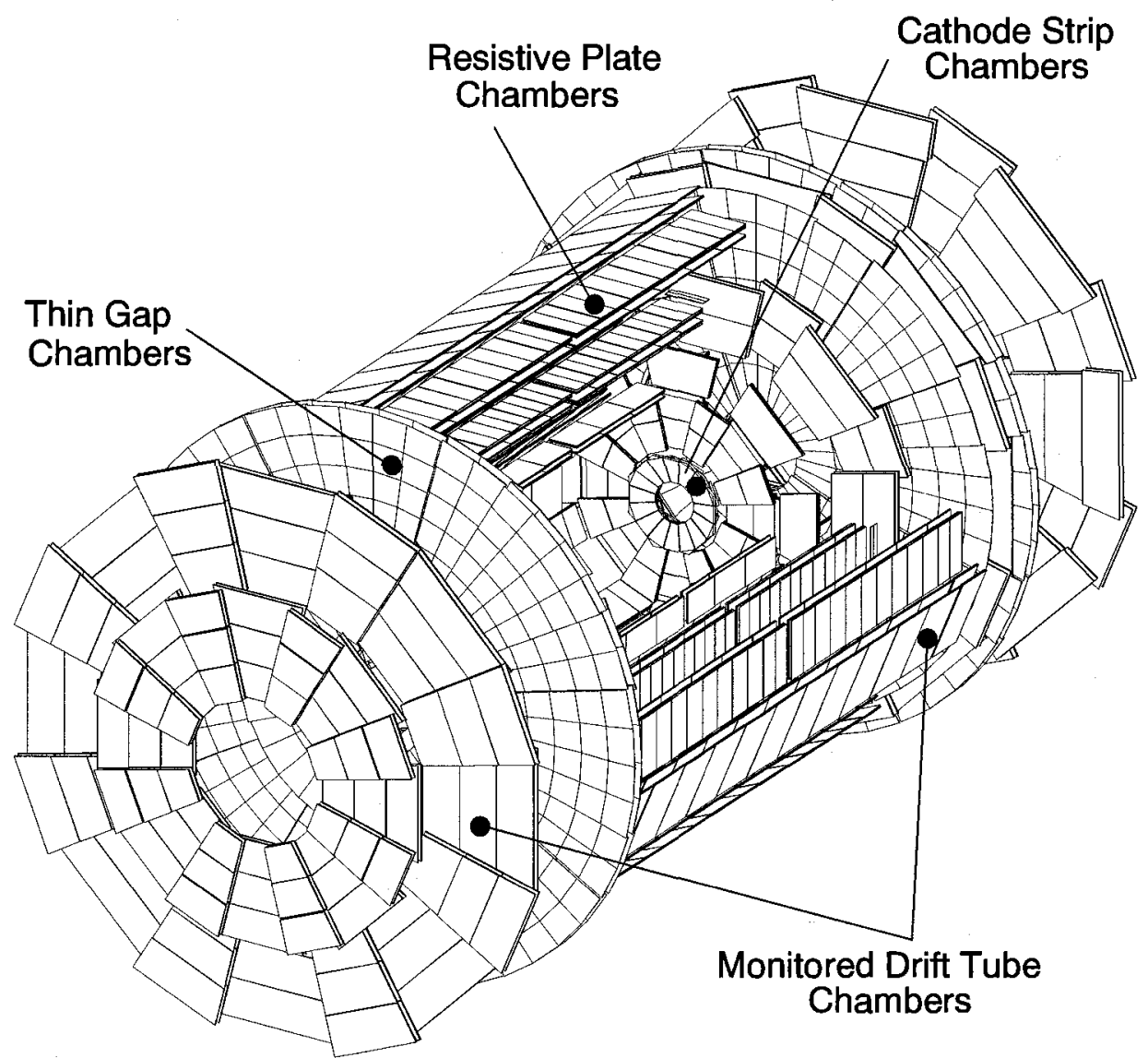

Figure 2.4: The ATLAS Muon System. 
coils, each individually housed in a cryostat measuring $25 \mathrm{~m}$ in length and $5 \mathrm{~m}$ in width. The two End-Cap Toroids (ECT) also have 8 coils that are housed in a single vessel and which are rotated $22.5^{\circ}$ in $\phi$ with respect to the BT coils to optimize the magnetic bending power and uniformity. The peak magnetic field provided by each toroid magnet is $\sim 4$ Tesla.

The Precision Measurements of muon tracks are achieved using the Monitored Drift Tubes (MDT) in the barrel region and the Cathode Strip Chambers (CSC) in the high $\eta$ range. The combination of the two detectors provides coverage up to $|\eta|<3$.

The Trigger System is composed of the Resistive Plate Chambers (RPC) and the Thin Gap Chambers (TGC) which provide a coverage of $|\eta|<2.4$. The RPC are used in the barrel and the TGC are used in the end-cap.

\subsection{Trigger and Data Acquisition}

The ATLAS trigger and data acquisition system is composed of three levels, as shown in Figure 2.5. The trigger is designed to reduce the data rate from the beam crossing rate of $40 \mathrm{MHz}$ to $\sim 200 \mathrm{~Hz}$. It must efficiently remove minimum-bias events, with a rejection factor of $\sim 10^{7}$, while retaining the hard-scattering events of interest. The following is a brief summary of each trigger level.

The Level-1 Trigger uses the muon trigger system in conjunction with coarse granularity information from the calorimeter system. The muon trigger provides information on the number of muon tracks and their transverse momentum and the calorimeter system provides information on electron/photon objects, hadronic jets and missing transverse energy. The overall level-1 trigger is processed by the central trigger processor which applies a trigger menu using both the muon and calorimeter information. In addition, the level-1 trigger defines Regions-of-Interests (RoIs) which are used as seeds for the subsequent trigger levels. RoIs are geographical coordinates in 


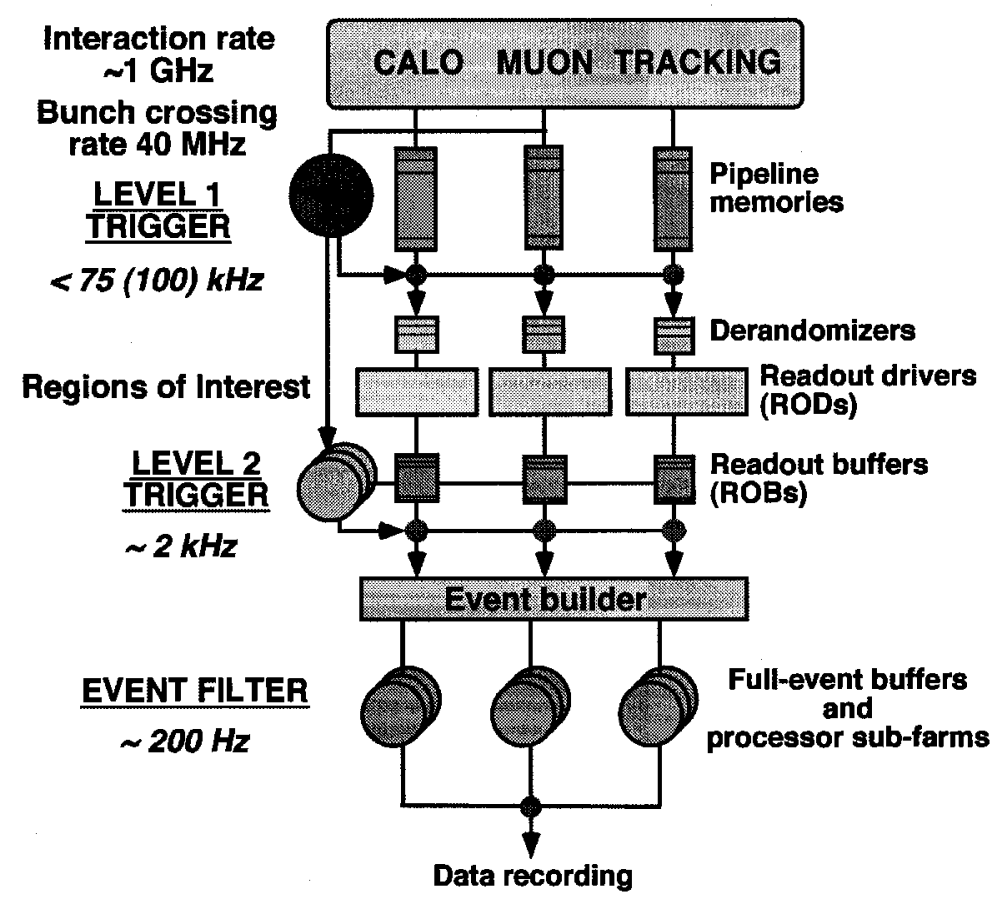

Figure 2.5: The ATLAS Trigger/DAQ System.

$\eta$ and $\phi$ with interesting features such as high $p_{T}$. The level-1 trigger is implemented at the hardware level and is designed to produce a trigger acceptance rate of $75 \mathrm{kHz}$ within a decision time of $\sim 2.5 \mu s$. Events which pass the level-1 trigger are read out from the front-end electronics onto the readout drivers and passed to the readout buffers.

The Level-2 Trigger is seeded by the level-1 Rols and has access to the full event data with full calorimeter granularity through the readout buffers. However, level-2 trigger elements generally only use data within a defined $\eta$ and $\phi$ window from the level-1 RoI seed. If the event passes the level-2 trigger then the data in the readout buffers is passed to the event-building system and subsequently to the event filter. The level- 2 trigger is designed to reduce the acceptance rate to $3.5 \mathrm{kHz}$ with a latency of $10 \mathrm{~ms}$.

The Event Filter uses more sophisticated selection algorithms similar to the offline software. The Event Filter is designed to reduce the acceptance rate to $200 \mathrm{~Hz}$ within 
an average decision time of a few seconds. Events which pass the event filter are moved to permanent storage. 


\section{Chapter 3}

\section{Principles of Shower Process and Calorimetry}

This chapter will summarize the process by which particles interact and lose energy in a detector and will also summarize the key principles of calorimetry.

\subsection{Shower Processes}

When a particle traverses matter, it will generally interact and lose energy. The following section briefly describes the different processes by which particles can lose their energy.

If a particle has sufficient energy, it will produce a cascade of particles known as showers. Showers are classified as electromagnetic or hadronic. The electromagnetic showers are cascades of particles produced by an incoming electron, positron, or photon. These showers are the easiest to understand because they only involve the electromagnetic force which is well understood and there are a limited number of possibilities for the shower development.

In contrast, hadronic showers are much more complicated because they involve the strong 
force, in addition to the electromagnetic force. As a consequence of including the strong force, there are more than 300 different reactions which contribute to the shower development [18]. Hadronic showers are thus inherently harder to model than their counterpart, the electromagnetic showers.

\subsubsection{Electromagnetic Showers}

This section provides a description of how electrons, positrons, and photons lose energy and a description of how a shower develops.

The electron ${ }^{1}$ has two primary mechanisms for energy loss within matter, as shown in Figure 3.1. They are ionization and bremsstrahlung. For example, ionization is the dom-

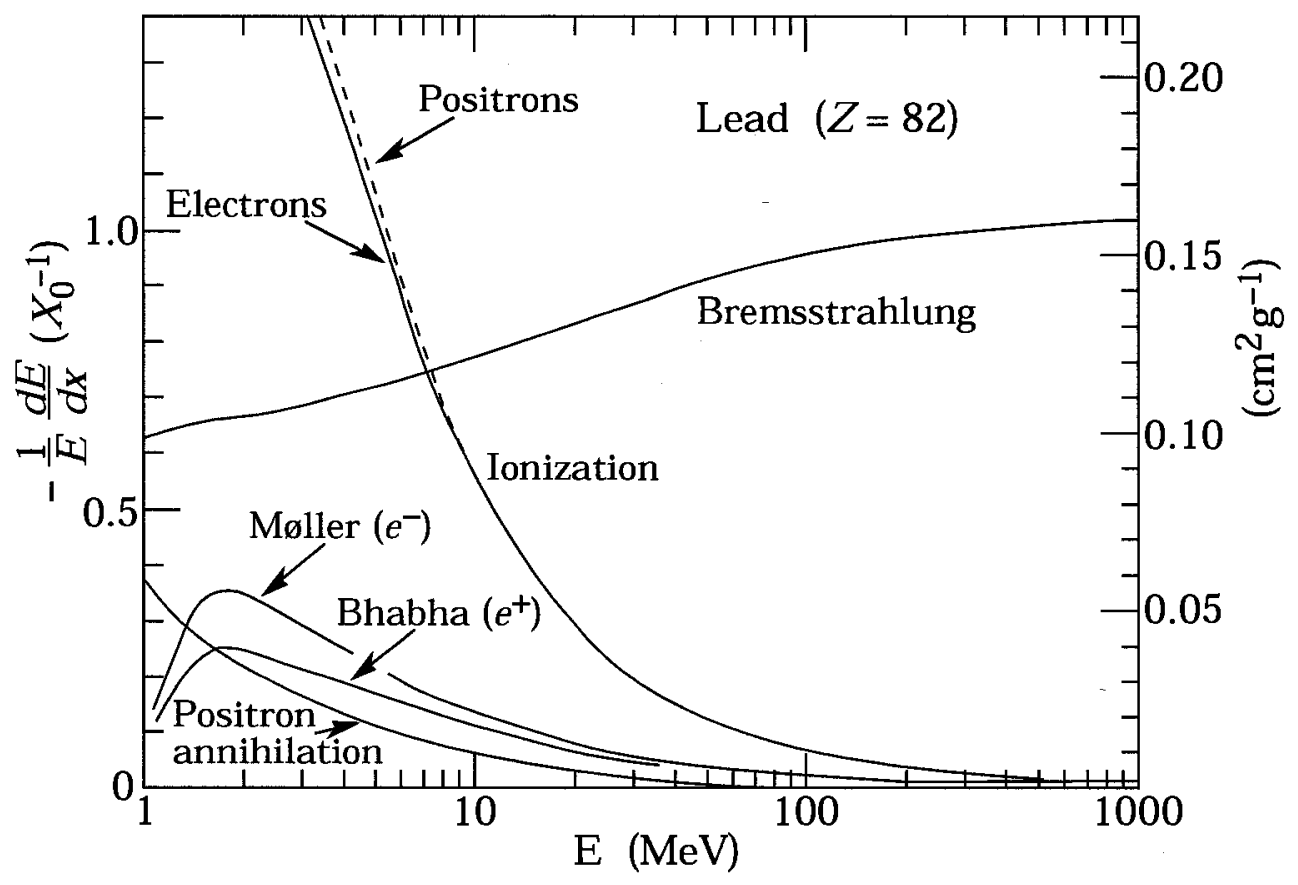

Figure 3.1: Fractional energy loss per radiation length of an electron or positron. Figure from reference [19].

inant mechanism at energies below $\sim 10 \mathrm{MeV}$ in lead. However, at energies larger than $\sim 10 \mathrm{MeV}$, bremsstrahlung is the dominant mechanism for energy loss. Bremsstrahlung

\footnotetext{
${ }^{1}$ Unless specifically stated, the electron will be used interchangeably with positron.
} 
is the production of electromagnetic radiation (photons) as a result of the interaction between the electron and the electric field of the nucleus. The point at which the energy loss from ionization and bremsstrahlung are roughly equal defines a key concept of the electromagnetic cascade, and is referred to as the critical energy, $E_{c}$. Below the critical energy, bremsstrahlung is no longer the dominant mechanism for energy loss and effectively terminates the electron's contribution to the shower development.

The photon has three dominant mechanisms for energy loss within matter, as shown in Figure 3.2. In lead, the photoelectric effect is important for photons with energies below

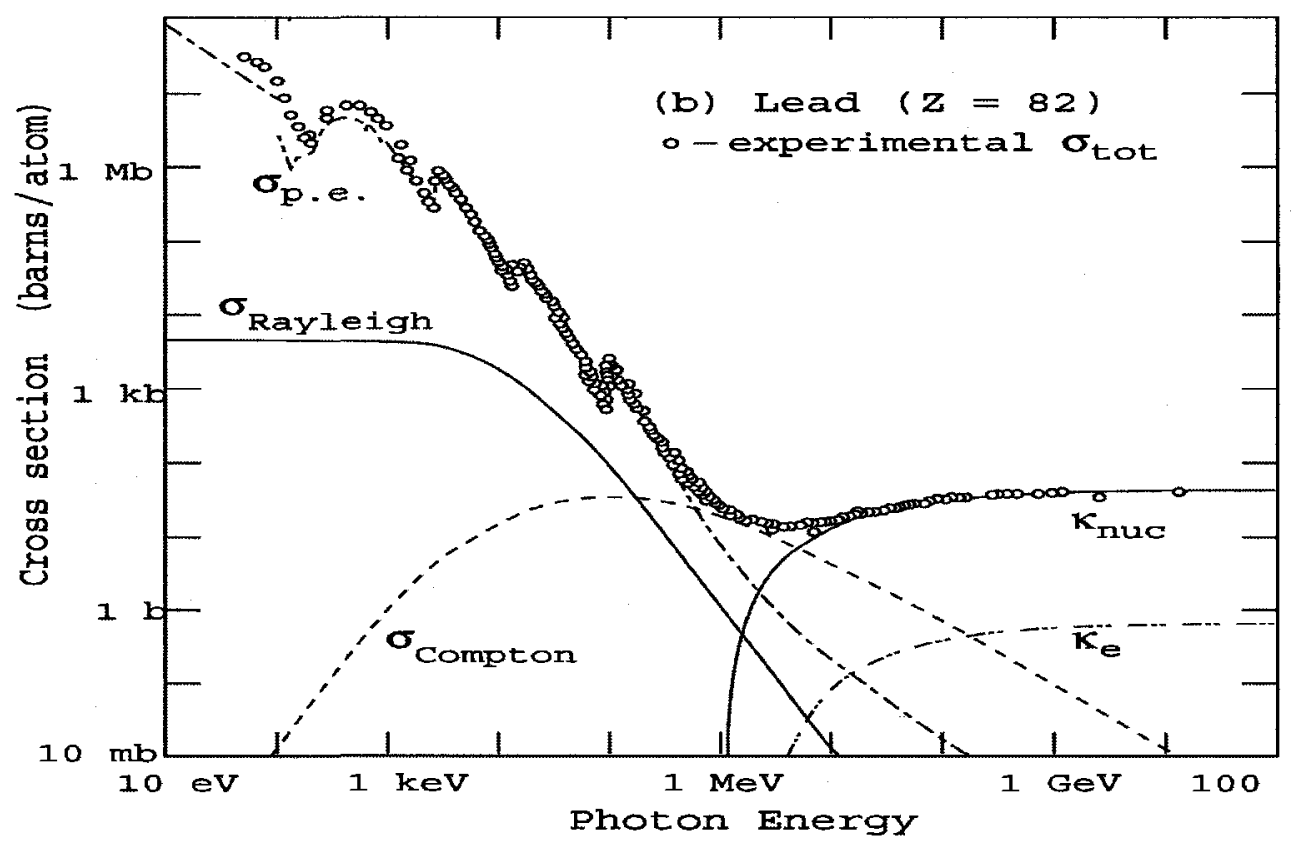

Figure 3.2: Photon cross-section showing the contributions of different processes. The three primary mechanisms are: the photoelectric effect $\left(\sigma_{\text {p.e. }}\right)$, Compton scattering $\left(\sigma_{C o m p t o n}\right)$, and pair production via nuclear field $\left(\kappa_{n u c}\right)$. Figure from reference [19].

$\sim 100 \mathrm{keV}$. The photoelectric effect is the absorption of the incoming photon by the atom resulting in the emission of an electron. This process requires that the energy of the incoming photon be larger than the binding energy of the electron in the atom. Once the energy exceeds $\sim 100 \mathrm{keV}$ up to $\sim 10 \mathrm{MeV}$, Compton scattering is the dominant mechanism in lead. Compton scattering is the exchange of 4-momentum from the incoming photon to 
the atomic electron, freeing the electron. At energies above $\sim 10 \mathrm{MeV}$, photons in lead start creating $e^{+} e^{-}$pairs in the presence of a nucleus. This process is called pair production.

At high energies, the two dominant mechanisms for energy loss are bremsstrahlung for electrons and pair production for photons. It is the interplay between these two mechanisms which results in the electromagnetic shower. A high-energy electron colliding into material will predominately produce a photon via the bremsstrahlung process. If the radiated photon has sufficient energy, it will create an $e^{+} e^{-}$pair. This cycle will continue as long as the energy of the electron or photon is above the critical energy. Below this energy, the photons are absorbed and the electrons will ionize the material in a calorimeter. It is the ionization of the active material that results in a detectable signal.

The scale used to characterize the longitudinal development of an electromagnetic shower is the radiation length $\left(X_{0}\right)$. The radiation length for high-energy electrons $(>1 \mathrm{GeV})$ is defined as the amount of material traversed by an electron in order to lose $63.2 \%$ of its energy via the bremsstrahlung process. This is related to the mean distance required for a highenergy photon to convert to an $e^{+} e^{-}$pair, $\frac{7}{9} X_{0}$. An approximate value of the radiation length is given by Equation 3.1 [19].

$$
X_{0}=\frac{716.4 \mathrm{gcm}^{-2} A}{Z(Z+1) \ln (287 / \sqrt{Z})}
$$

Here, $\mathrm{Z}$ and $\mathrm{A}$ are the numbers giving the atomic number and atomic weight of the material. If the material is a mixture of different elements, the radiation lengths can be approximated by using Equation 3.2 [19].

$$
\frac{1}{X_{0}}=\sum \frac{w_{j}}{X_{j}}
$$

Here, $w_{j}$ and $X_{j}$ represent the fractional weight and radiation length of the $j$ th element. The longitudinal shower information can be determined by using the combination of the 
radiation length and the critical energy. From data, the longitudinal shower maximum can be estimated in radiation lengths using the empirical Equation 3.3 for energies between 2 and $300 \mathrm{GeV}[20]$.

$$
t_{\max }=3.9+\ln E_{i}
$$

Here, $E_{i}$ is the initial energy in $\mathrm{GeV}$. In addition, the longitudinal shower development containing $95 \%$ of the shower can be estimated in radiation lengths using Equation 3.4.

$$
t_{95}=t_{\max }+0.08 Z+9.6
$$

The transverse development of a shower is characterized by the Molière radius $\left(R_{M}\right)$ which is proportional to the radiation length and is estimated using Equation 3.5 [19].

$$
R_{M}=m_{e} c^{2} \sqrt{4 \pi / \alpha} \frac{X_{0}}{E_{c}}=21.2 \mathrm{MeV} \frac{X_{0}}{E_{c}}
$$

Here, $m_{e}$ is the mass of the electron, $c$ is the speed of light, $\alpha$ is the fine structure constant, and $E_{c}$ is the critical energy in $\mathrm{MeV}$. The source of the transverse shower spread is primarily due to multiple scattering and photons produced from the bremsstrahlung process which travel far from the initial shower axis. Roughly $95 \%$ of a shower is contained transversely in a cylinder with a radius of $2 R_{M}$.

\subsubsection{Hadronic Showers}

Hadronic showers are similar to electromagnetic showers in that the interaction of the initial particle produces secondary particles. However, hadronic showers result from the strong force, adding numerous processes in the shower development. Hadronic showers contain within them a purely electromagnetic component, which results from the production of $\pi^{0} \mathrm{~s}$ which subsequently decay into two photons. The photons in turn produce an electromagnetic shower component. The fraction of a hadronic shower which is electromagnetic can greatly fluctuate from event to event due to the statistics of $\pi^{0}$ production. 
The behaviour of the electromagnetic portion of a hadronic shower is governed by the physics described in Section 3.1.1. A general expression for the fraction of the electromagnetic component in a hadronic shower is provided by Gabriel et al. [21] and can be calculated using the power law Equation 3.6.

$$
f_{e m}=1-\left(\frac{E_{i}}{E_{h}}\right)^{k-1}
$$

Here, $E_{i}$ is the initial energy, $E_{h}$ is the average energy needed to produce a pion $(\sim 1$ $\mathrm{GeV})$, and $k$ is a constant which has been experimentally measured to be $\sim 0.8$ [22].

The purely hadronic component is more complicated and not as well understood. As with electrons, charged hadrons can lose their energy through ionization. In addition, high-energy hadrons can interact with an atomic nucleus creating a nuclear spallation reaction. The spallation reaction is comprised of two stages, a fast intranuclear cascade followed by the evaporation stage. The fast intranuclear cascade involves a collision between the initial hadron and a nucleon within the nucleus. If the energy transfer is sufficiently high, these collisions may create light mesons which escape the nucleus contributing to the hadronic shower. Otherwise, the nucleons will remain in the nucleus and distribute their energy via the evaporation stage. The evaporation stage involves the de-excitation of the nucleus by emitting nucleons, predominately neutrons, and photons in successive evaporation steps until the excitation energy is less than the binding energy of one nucleon. During the evaporation stage, a fraction of the energy of the initial particle goes into processes such as neutron absorption, which are not measurable in a calorimeter. This results in variable amounts of shower energy that can not be seen. Although a correction can be applied for this, the fluctuation in lost energy degrades the resolution that can be achieved.

Similar to the electromagnetic shower, the hadronic shower has a distance scale which 
is determined by the nuclear interaction length $\left(\lambda_{i n t}\right)$. The nuclear interaction length is defined as the average distance a hadron travels before a nuclear interaction occurs. The nuclear interaction length can be calculated using the approximation given in Equation $3.7[19]$.

$$
\lambda_{\text {int }}=35 \mathrm{~g} \mathrm{~cm}^{-2} A^{1 / 3}
$$

Here, $A$ is the atomic weight of the material. If the material is a mixture of different elements, the nuclear interaction length can be calculated in the same way as for the radiation length using Equation 3.2. The longitudinal hadronic shower maximum can be approximated in units of nuclear interaction length using Equation 3.8 .

$$
t_{\max }=0.9+0.36 \ln E_{i}
$$

Here, $E_{i}$ is the initial energy in GeV. Approximately $95 \%$ of the transverse shower is contained within a cylinder with a radius of one nuclear interaction length and the shower width does not depend strongly on the initial energy.

\subsection{Calorimetry}

In this section, the properties of sampling calorimetry techniques are described. A calorimeter, in particle physics, is a device which measures the total deposited energy by an incoming particle within the detector volume.

\subsubsection{Sampling Calorimetry and Sampling Fraction}

All calorimeters in ATLAS are sampling calorimeters, that is, they are divided into a series of absorbing and active layers, as shown in Figure 3.3. A calorimeter system is generally composed of electromagnetic calorimeters followed by hadronic calorimeters. This division provides optimal performance of the calorimeter system, such as energy resolution and particle identification. Electromagnetic calorimeters are designed to provide optimal 


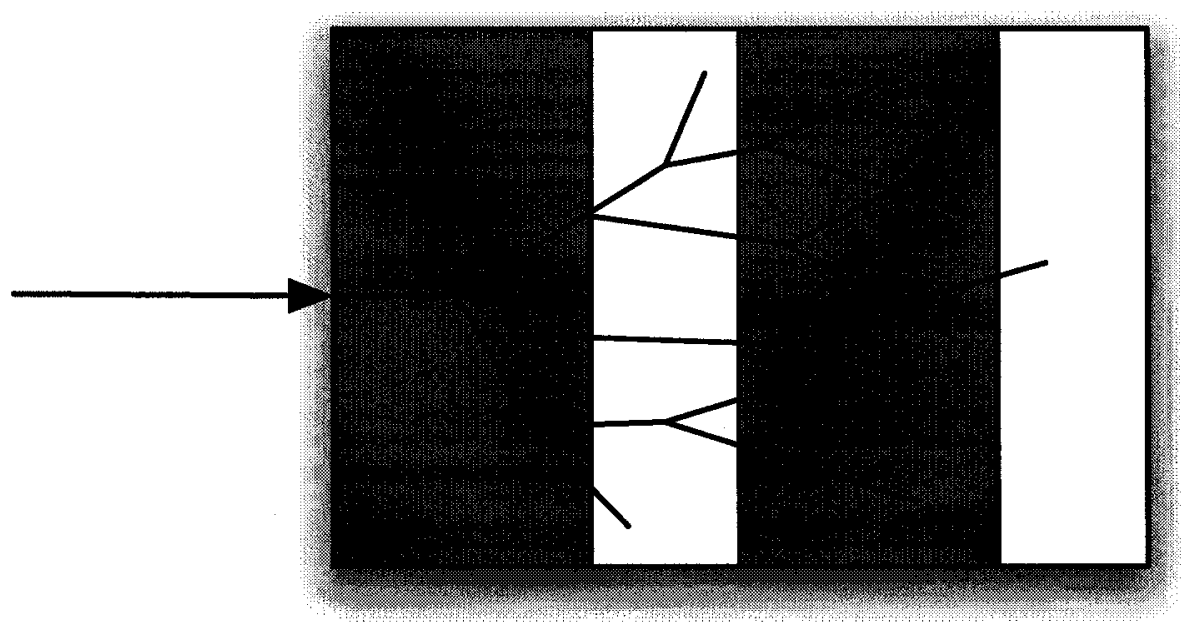

Figure 3.3: Example of a particle shower in a sampling calorimeter. The gray and white boxes represent the absorber and active layers, respectively.

measurement of energy for electrons, positrons, and photons and are generally less dense than their hadronic counterparts. Hadronic calorimeters are usually denser in order to contain showers within a reasonable volume. The active layer of the calorimeter provides the actual energy measurement and the absorbing layer provides the material for shower development. Therefore, a sampling calorimeter only measures a fraction of the energy of the shower. Equivalently, one could say a "sample" is taken of the energy deposited. Since sampling calorimeters only measure a fraction of the energy, the energy deposited in a single layer of active material is given in Equation 3.9.

$$
\Delta E=\left.\frac{d E}{d x}\right|_{a} d_{a}
$$

Here, $\left.\frac{d E}{d x}\right|_{a}$ is the energy deposited in the active material per unit distance and $d_{a}$ is the width of the active material. Therefore, the total amount of energy that is measured is $E_{m}=N \Delta E$, where $N$ is the number of charged tracks in all the active layers. In order to determine the total energy deposited in a sampling calorimeter the relationship between the measured energy and the total energy deposited must be known. The sam- 
pling fraction provides the relationship between the measured energy and the total energy deposited as shown in Equation 3.10.

$$
E_{m}=E_{d} S
$$

Here, $E_{m}$ and $E_{d}$ are the measured and deposited energy throughout the calorimeter and $S$ is the sampling fraction. When determining the sampling fraction from data one should be aware that this not only reflects the material and geometry of the calorimeter but also the electronics from the readout chain and the reconstruction algorithm used.

\subsubsection{Energy Resolution}

The energy resolution is the precision with which a calorimeter can measure the energy of an incoming particle. The energy resolution is usually parameterized using Equation 3.11 .

$$
\frac{\sigma}{E}=\frac{A}{\sqrt{E}} \oplus B \oplus \frac{C}{E}
$$

Here, the first term, $A / \sqrt{E}$, is the stochastic term. The second term, $B$, is known as the constant term and originates from various imperfections such as calibration errors and construction non-uniformities. The final term, $C / E$, is energy-independent and is usually associated with the readout noise. The energy resolution can also be parameterized using Equation 3.12 if the noise injected into the calorimeter is determined separately.

$$
\frac{\sigma}{E}=\frac{A}{\sqrt{E}} \oplus B
$$

\subsubsection{Compensation}

Depending on the materials chosen to build a calorimeter, the energy response between electrons and hadrons can vary. Compensation refers to the attempt to compensate for differences between the response of electrons and hadrons in a given calorimeter. In most calorimeters, the response to hadrons is smaller than it is to electrons. In its simplest form, 
compensation is an attempt to equalize the response to the electromagnetic and hadronic components of a shower to provide a consistent response. There are two ways to achieve compensation: calorimeter design and offline software. The first involves selecting detector materials which either suppress the electromagnetic component or enhance the nonelectromagnetic component. One should be aware that the choice of the materials can also lead to overcompensation. Overcompensation occurs when the hadronic response is larger than the electromagnetic response. An alternate approach is to correct for the difference by using offline software. This method involves corrections based on the shower profile for both electrons and hadrons. This is largely motivated by the difference in the density of deposited energy between the electromagnetic and hadronic showers. The level of non-compensation in calorimeters is characterized by the $e / h$ ratio. However, the $e / h$ ratio cannot be measured directly but rather indirectly, using Equation 3.13 and the $e / \pi$ ratio from data [22].

$$
\frac{e}{h}=\frac{1-f_{e m}(E)}{\pi / e(E)-f_{e m}(E)}
$$

Here, $f_{e m}(E)$ is the average electromagnetic energy fraction which can be determined using Equation 3.6. It should be noted that all ATLAS calorimeters are non-compensating and require some offline correction. 


\section{Part I}

\section{The ATLAS Forward Calorimeter 2003 Test Beam}




\section{Chapter 4}

\section{Introduction}

Presented in this part of the thesis is a study of the performance of the ATLAS Forward Calorimeter (FCal) modules using data taken in 2003. In Sections 4.1 and 4.2, the motivation for and the design of the ATLAS FCal modules is presented. The remainder of this chapter describes the goals and setup of the 2003 test beam. Chapter 5 summarizes the data treatment and Chapter 6 explains how the signal amplitude for a single readout cell energy was determined. Finally, Chapter 7 presents the methods used in the test beam analysis and the results obtained for electron and hadron data.

\subsection{Motivation for the ATLAS FCal Modules}

As stated in Chapter 1, the main goal of the LHC is to understand the electroweak symmetry breaking mechanism. Within the Standard Model of particle physics, the Higgs mechanism provides a model which predicts the existence of the Higgs boson. The second largest production mode for the Higgs boson, after gluon-gluon fusion, is Vector Boson Fusion (VBF). This production mode is characterized by two jets, the spatial distribution of which is peaked in the forward direction. This can be seen in Figure 4.1 which shows the $\eta$ distribution for the two forward jets. The VBF production is crucial for Higgs studies given that backgrounds can be heavily suppressed using the information from the two 


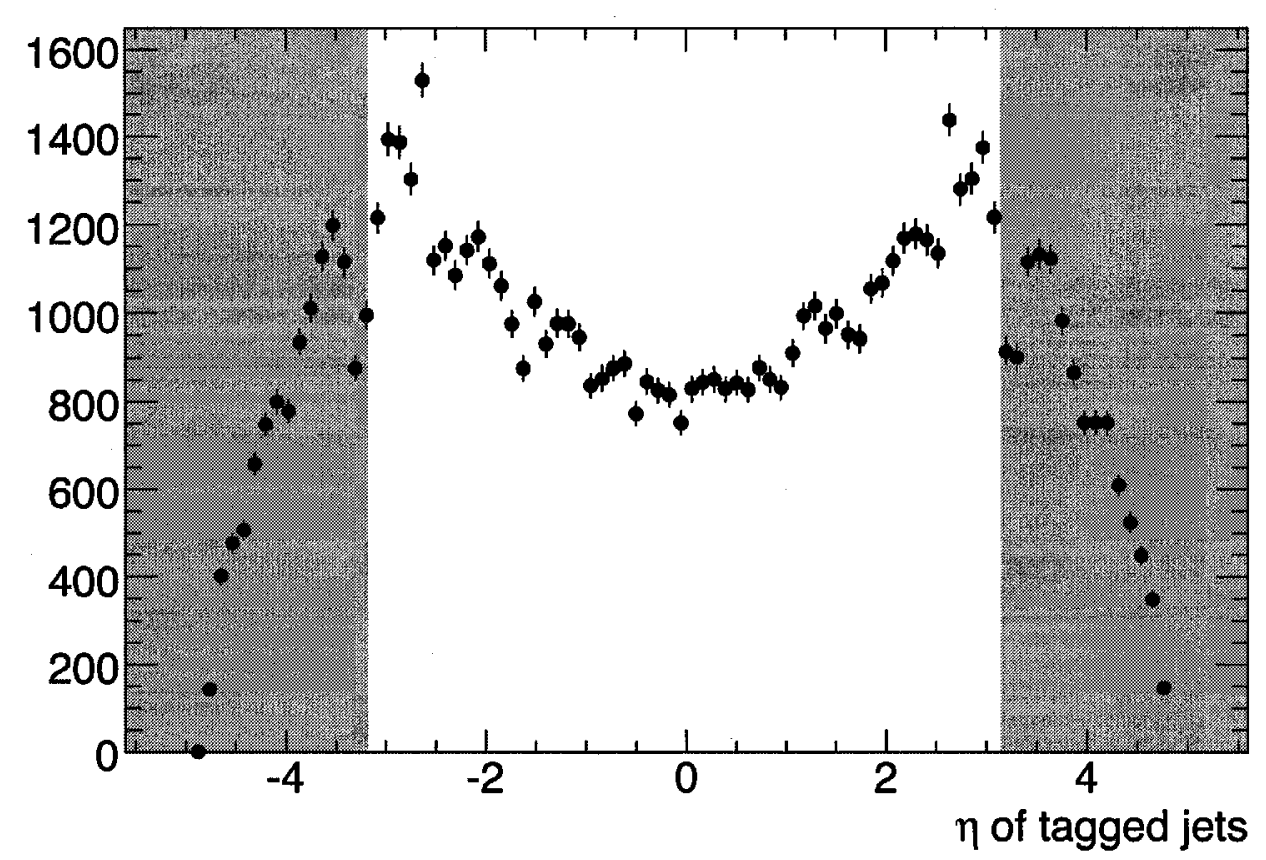

Figure 4.1: The $\eta$ distribution of the two tagged jets for a $130 \mathrm{GeV}$ mass Higgs produced by VBF. The shaded area represents the FCal detector coverage.

tagged jets. In addition to jets, several searches for physics beyond the Standard Model, such as the invisible Higgs, require a good reconstructed missing transverse energy. In order to address both jet tagging efficiency and missing transverse energy, a hermetic calorimeter system with a large $\eta$ acceptance is required.

The additional coverage provided by the FCal allows for an increase of approximately $54 \%$ in the efficiency of tagging both jets from VBF production, for a $130 \mathrm{GeV}$ mass Higgs. In order to measure these jets and other jets of interest, the resolution requirements for the transverse energy of the jets, $E_{T}$, was set at $\Delta E_{T} / E_{T}<10 \%$ for $E_{T}>25 \mathrm{GeV}$. Jets below this minimum $E_{T}$ of $25 \mathrm{GeV}$ are expected to be lost due to pile-up ${ }^{1}$. The $E_{T}$ requirement places limits on the FCal jet energy resolution of $\Delta E / E<7 \%$ and on the FCal angular resolution of $\Delta \theta / \theta<7 \%$ for jet energies above $250 \mathrm{GeV}$. At the highest $\eta$, the

\footnotetext{
${ }^{1}$ Pile-up arises from proton-proton collisions that are not part of the event of interest and from earlier proton-proton collisions.
} 
angular resolution dominates. In addition, by extending the calorimeter's coverage the missing transverse energy, $\mathbb{E}_{T}$, resolution is improved. However, the tails on the energy distribution due to the intrinsic performance of each detector must be well understood in order to fully understand any $\mathbb{E}_{T}$ result. These tails can result in the production of a fake $\mathbb{E}_{T}$ signal. A fake $\mathbb{F}_{T}$ signal can also be produced by other calorimeter problems such as mis-calibration and energy loss in regions where there are no instrumented detectors.

\subsection{The ATLAS FCal Calorimeter Design}

The Large Hadronic Collider (LHC) will provide 14 TeV center-of-mass proton-proton collisions at a design luminosity of $10^{34} \mathrm{~cm}^{-2} \mathrm{~s}^{-1}$. The high energy and high collision rates of the LHC result in very high particle fluxes, particularly in the forward direction, that present some challenges and constraints on the design of the detectors. In the original ATLAS design, the FCal modules were placed approximately $15 \mathrm{~m}$ from the Interaction Point (IP) in order to reduce radiation damage caused by the high particle density near the IP. However, several physics studies showed advantages to integrating the FCal into the endcap calorimeter system. These advantages include minimizing the un-instrumented transitions between calorimeters (cracks), reducing the dead material in front of the calorimeter, and providing superior shielding for the muon system. Therefore, the final ATLAS FCal design integrates the FCal modules into the endcap calorimeter system and extends the pseudo-rapidity range of the Liquid Argon (LAr) calorimeter system from approximately 3.2 to 5 . Figure 4.2 provides a schematic diagram of the ATLAS endcap calorimeter system. The ATLAS FCal system is composed of three fully instrumented modules, which are referred to as FCal 1, FCal 2 and FCal 3 and a fourth un-instrumented brass module (plug), as shown in Figure 4.3. The FCal modules are positioned longitudinally one behind the other. The FCal 1 module is the closest to the IP, located at a distance of 4.7 meters from the IP, followed by FCa1 2, FCal 3, and the plug. The FCal 1 module is composed mainly of copper and has a Molière radius of $17.1 \mathrm{~mm}$. Copper was chosen to ensure 


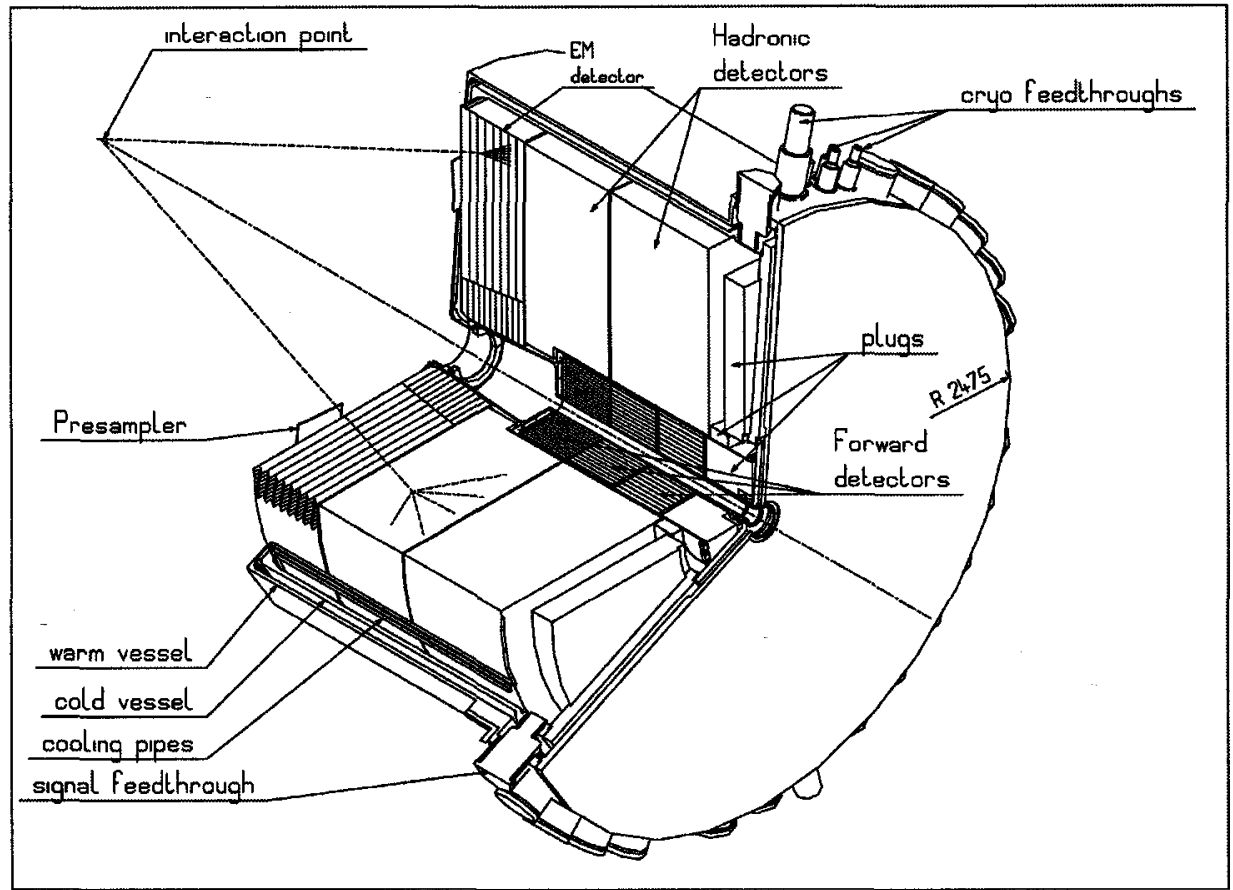

Figure 4.2: Schematic diagram illustrating the endcap cryostat which houses the FCal (labelled Forward detectors) modules along with the EMEC and HEC (labelled EM detector and Hadronic detector respectively.).

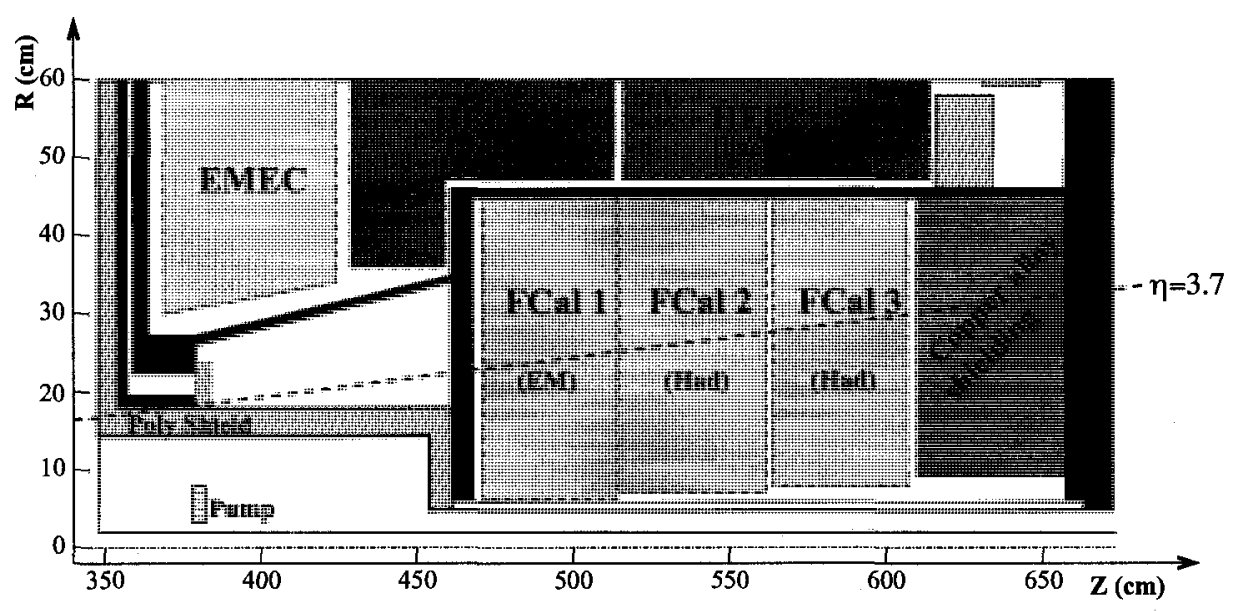

Figure 4.3: Schematic diagram of the FCal modules in the ATLAS Endcap cryostat. The dashed line corresponds to the particle trajectory for $\eta=3.7$. 
a uniform response as the electromagnetic shower width in these copper modules was large enough to cover several electrodes. The use of copper also provided efficient heat transfer to prevent boiling in the liquid argon. In contrast, FCal 2 and FCal 3 modules are composed predominately of tungsten and are designed to contain hadronic particle showers within a limited space. The geometrical properties and mass of the instrumented ATLAS FCal detector are listed in Table 4.1. Finally, the plug provides additional mate-

\begin{tabular}{|c|c|c|c|}
\hline Module & FCal1 & FCal2 & FCal3 \\
\hline \hline Absorber Material & Copper & Tungsten & Tungsten \\
\hline Inner Radius [mm] & 72.3 & 78.8 & 85.7 \\
\hline Outer Radius [mm] & 449.4 & 449.4 & 449.4 \\
\hline Mass [kg] & 2130 & 3830 & 3700 \\
\hline Depth [mm] & 444.1 & 444.1 & 444.1 \\
\hline Depth $\left[X_{0}\right]$ & 27.6 & 89.7 & 88.0 \\
\hline Depth $[\lambda]$ & 2.65 & 3.62 & 3.55 \\
\hline Electrode-to-electrode $[\mathrm{mm}]$ & 7.50 & 8.18 & 9.00 \\
\hline
\end{tabular}

Table 4.1: The ATLAS FCal detector geometrical properties and mass. The dimensions are for the detector at LAr temperature $(\sim 90 \mathrm{~K})$. Here $X_{0}$ and $\lambda$ are the radiation and nuclear interaction lengths, respectively.

rial to prevent punch-through of energetic secondary particles that might otherwise enter the muon system. The plug has the same geometrical size as the other modules with the exception of a slightly larger inner radius at $94.7 \mathrm{~mm}$. The FCal modules have a unique geometry, as shown in Figure 4.4, which was chosen to provide a good response in the high flux environment that will occur at the LHC. This large flux which is due to minimum bias ${ }^{2}$ events and pile-up can cause ion build up in the LAr gaps. Reducing the gaps to approximately an eighth of the size of traditional LAr detectors of $\sim 2 \mathrm{~mm}$ to $\sim 0.25 \mathrm{~mm}$ solves this problem. To achieve these small liquid argon gaps, a conventional design with parallel plates perpendicular to the beamline was abandoned. Instead, a novel matrix design of cylindrical electrodes oriented parallel to the beamline was adopted. Electrodes are composed of a copper cylindrical outer tube (cathode) which houses an absorber rod (anode). The gaps between the rods and the tubes are maintained by a PEEK fiber he-

\footnotetext{
${ }^{2}$ Minimum bias events are customarily defined as non-single diffractive inelastic interactions and are characterized by low- $p_{T}$ parton scatterings.
} 


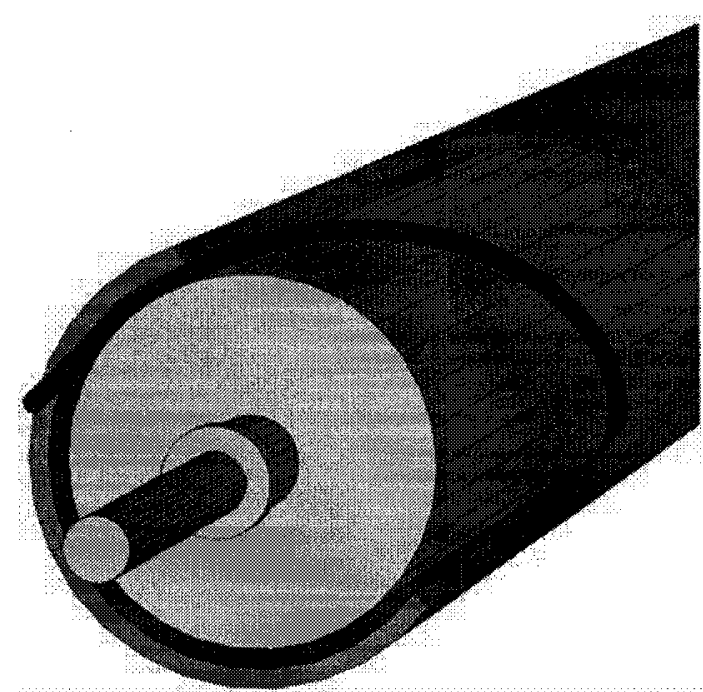

(a) Schematic diagram of a single FCal electrode structure.

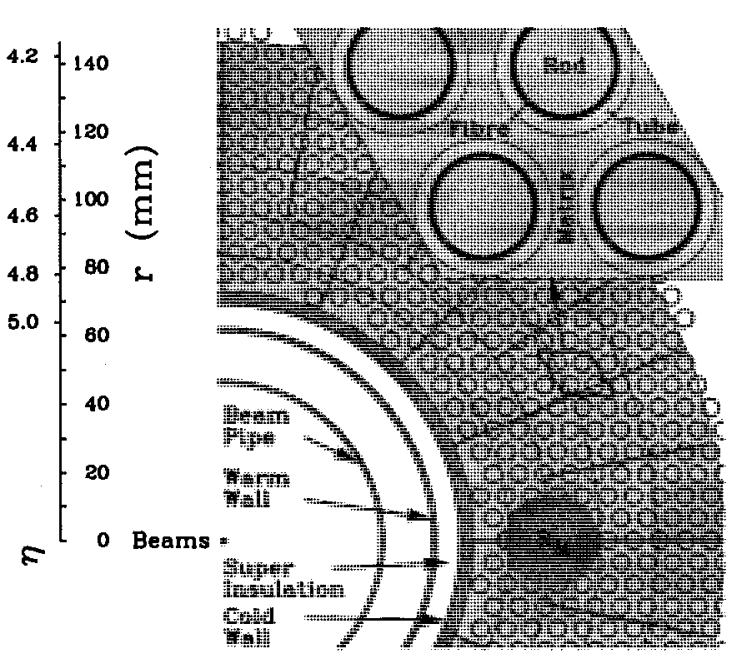

(b) Front face of the FCal 1 module illustrating the electrode arrangement with an example of an electrode grouping. The Molière radius in FCal 1 is shown as the circle labeled $R_{M}$.

Figure 4.4: Schematic diagrams illustrating the unique detector design of the FCal modules.

lically wound around the absorber rod, as shown in Figure 4.4(a). The space between the outer tube and the rod is filled with LAr. The electrodes are arranged in a hexagonal pattern, as shown in Figure 4.4(b), with a small electrode-to-electrode distance of $7.5 \mathrm{~mm}$ for FCal 1. The electrode-to-electrode distance for all three modules is given in Table 4.1. The fine transverse granularity of the FCals can be exploited to characterize the particle shower development and is used in one of the hadronic calibration schemes discussed in this thesis. Electrodes are ganged into groups of 4, 6 and 9 for FCal 1, FCal 2, and FCal 3, respectively. In most cases, four of the resulting electrode groups are summed on a transformer to a single readout channel. The transformer is a passive device used to match the impedance of the four coaxial cables to the pigtail cable while keeping the electronic noise the same as for a single tube group. However, because of geometrical constraints, some of the readout channels at the inner and outer edge are not summed. The High Voltage (HV) is distributed to the calorimeter on the summing board. Four independent HV lines service each summing board. The connection pattern between the HV lines and the tube 
groups was designed to minimize adjacent dead tube groups in the event of a HV failure. Once a signal is summed, it is sent to the cryostat feedthrough lines. All of these elements are located in the cryostat and are referred to as the cold electronics, illustrated in Figure 4.5 .

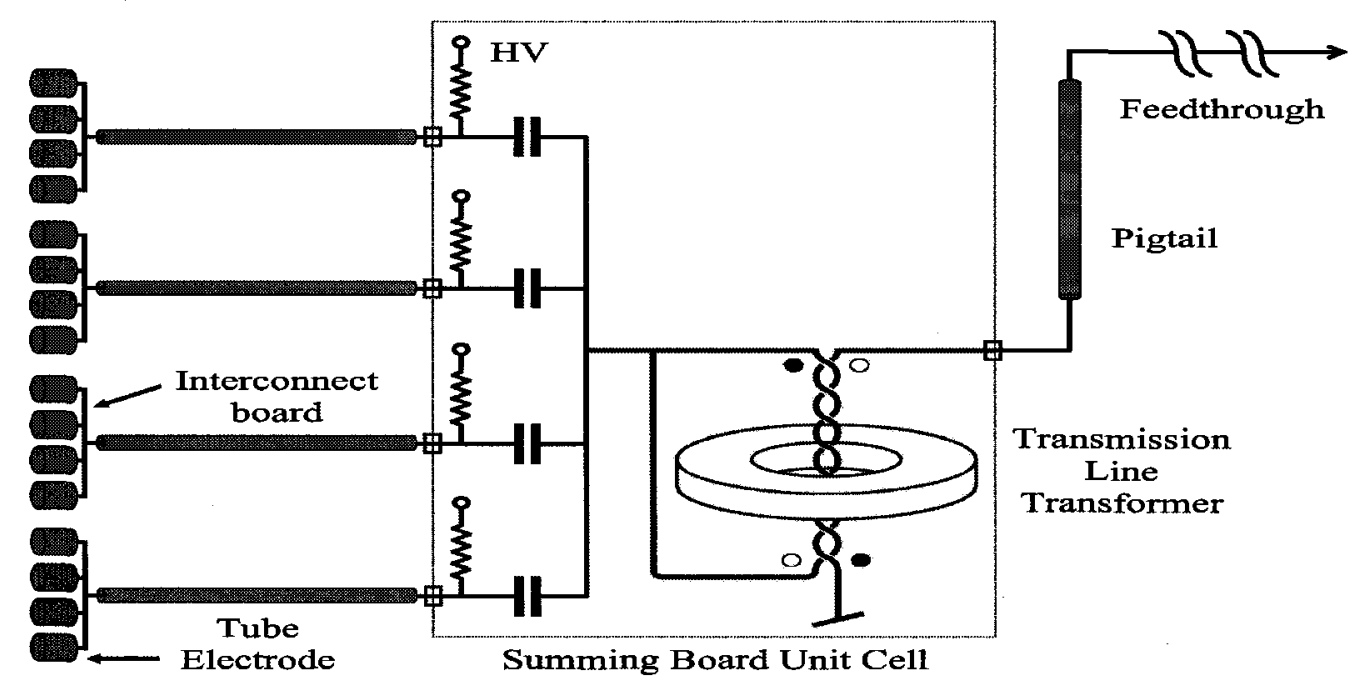

Figure 4.5: Schematic diagram of the cold electronics. Starting from the left, the electrodes are ganged together to create an electrode group. Afterwards, the groups are summed together on a transformer (summing board). Finally, the raw signal is passed to the feedthrough which connects the signal lines to the FEB.

Leaving the cryostat, the signal is passed to the Front-End Board (FEB). The FEB electronics amplify and shape the signal in three gain modes which are sampled continuously every 25 ns. The pulse shape in the FCal modules is generated by a charged particle which ionizes the active medium (LAr). The initial pulse shape caused by the ionized LAr is triangular with a sharp rise time followed by a linear drop. After passing through the electronics, the signal peak is rounded, followed by a negative part of the wave form, as shown in Figure 4.6. In the case of the data analyzed for the 2003 test beam, a total of seven samples were collected per readout channel. The gain mode of the signal was selected using a digital threshold based on the amplitude of the $4^{\text {th }}$ time sample. The cabling for the FCal modules is organized into 16- $\phi$ slices per module. Within a $\phi$ slice, the 


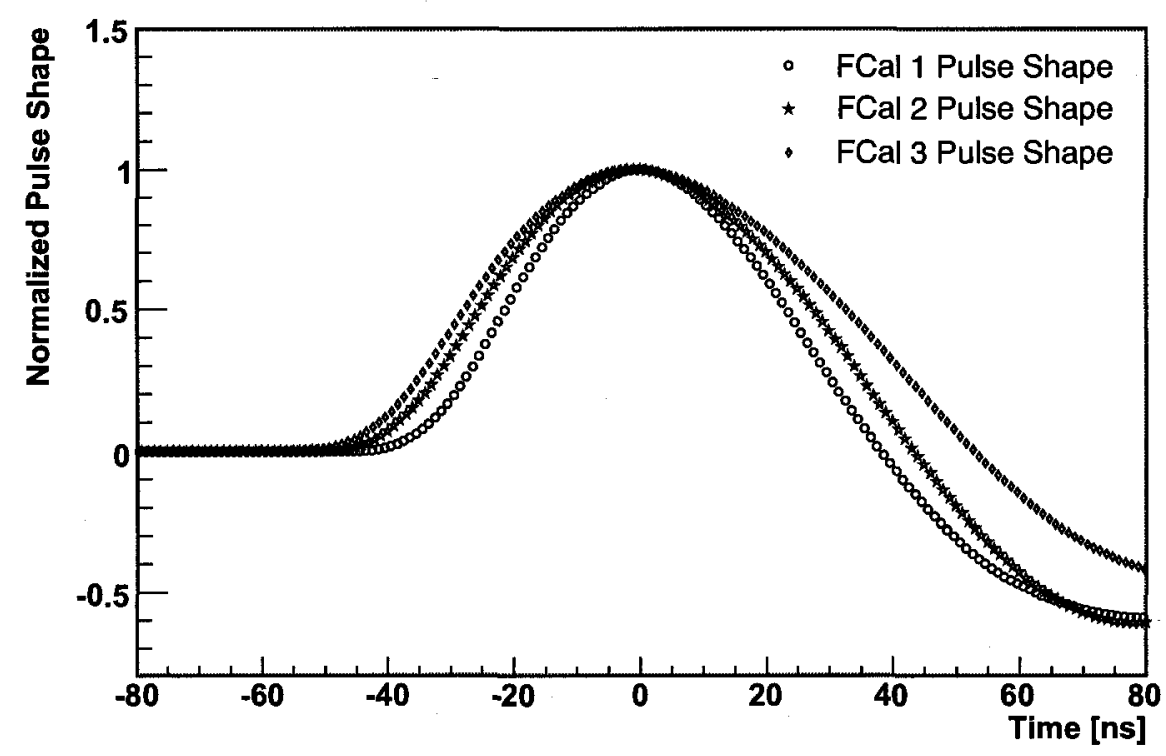

Figure 4.6: Examples of ionizing pulse shapes after electronic shaping for each of the three FCal modules.

cabling is divided into 4, 2, and $1 \eta$-slices for FCal 1, FCal 2, and FCal 3, respectively. Up to 64 readout channels are instrumented within an $\eta \times \phi$ section.

\subsection{Test Beam Goals and Setup}

For the 2003 test beam, the final ATLAS FCal modules, FCal 1, 2 and 3, were placed in a cryostat in the $\mathrm{H} 6$ test beam area at CERN. These modules were exposed to beams of electrons, pions, and muons within the energy range $5 \mathrm{GeV} \leqslant \mathrm{E} \leqslant 200 \mathrm{GeV}$ delivered by a secondary beam line of the CERN SPS. Downstream from the last bending magnet of the beam line, there were several scintillation counters and multi-wire proportional chambers followed by the cryostat containing the FCal modules. Behind the cryostat there was a warm tailcatcher, a concrete beam stop, and a muon scintillation counter, as shown in Figure 4.7.

There were two primary goals for the 2003 test beam. The first goal was to determine the 


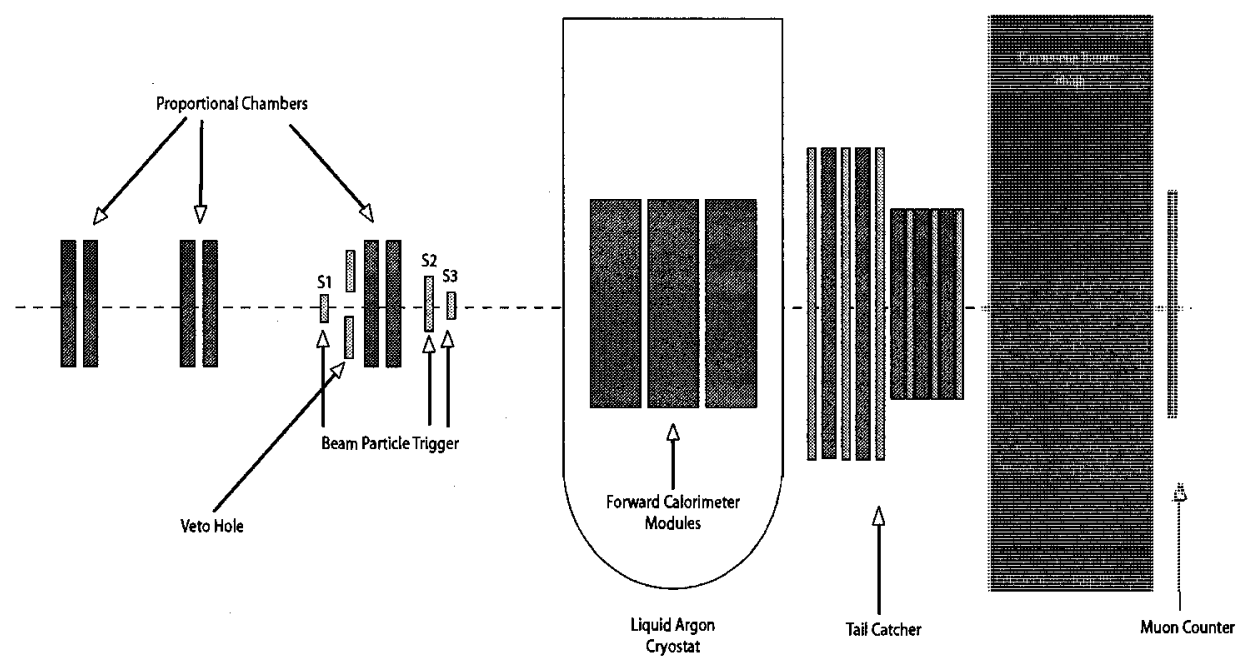

Figure 4.7: Schematic view of the FCal 2003 beam line. The vertical scale has been exaggerated compared to the horizontal scale for clarity.

optimal performance properties of the FCal modules, such as the energy scale, the energy resolution, and the linearity. This aspect of the test beam included a measurement of the intrinsic detector performance and the expected detector performance in the final ATLAS configuration with additional dead material in front of the FCal. The second goal of the test beam program was to determine the detector performance at the inner edge, in particular the effect of energy loss in the beam hole on the reconstructed energy for jets and $\mathbb{E}_{T}$. To achieve these goals, five beam impact points were used, as shown in Figure 4.8. A wide beam was used to average out the energy dependence on the beam particle impact point [23]. This dependence arises from the fact that the electrodes are oriented along the beam line. The measured beam energy which depends on the ionization in the LAr gap will vary depending on whether the beam initially impacts the liquid argon or the absorber. The $4 \mathrm{~L}$ position had minimal upstream material to measure the intrinsic properties of the FCal detectors. In addition, its location maximizes shower containment in the FCal modules. At the $4 \mathrm{H}$ position, additional dead material was introduced in front of the modules to reproduce the ATLAS environment. The additional dead material was intended to simulate material such as the cryostat bulkhead and polyethylene shielding, as 


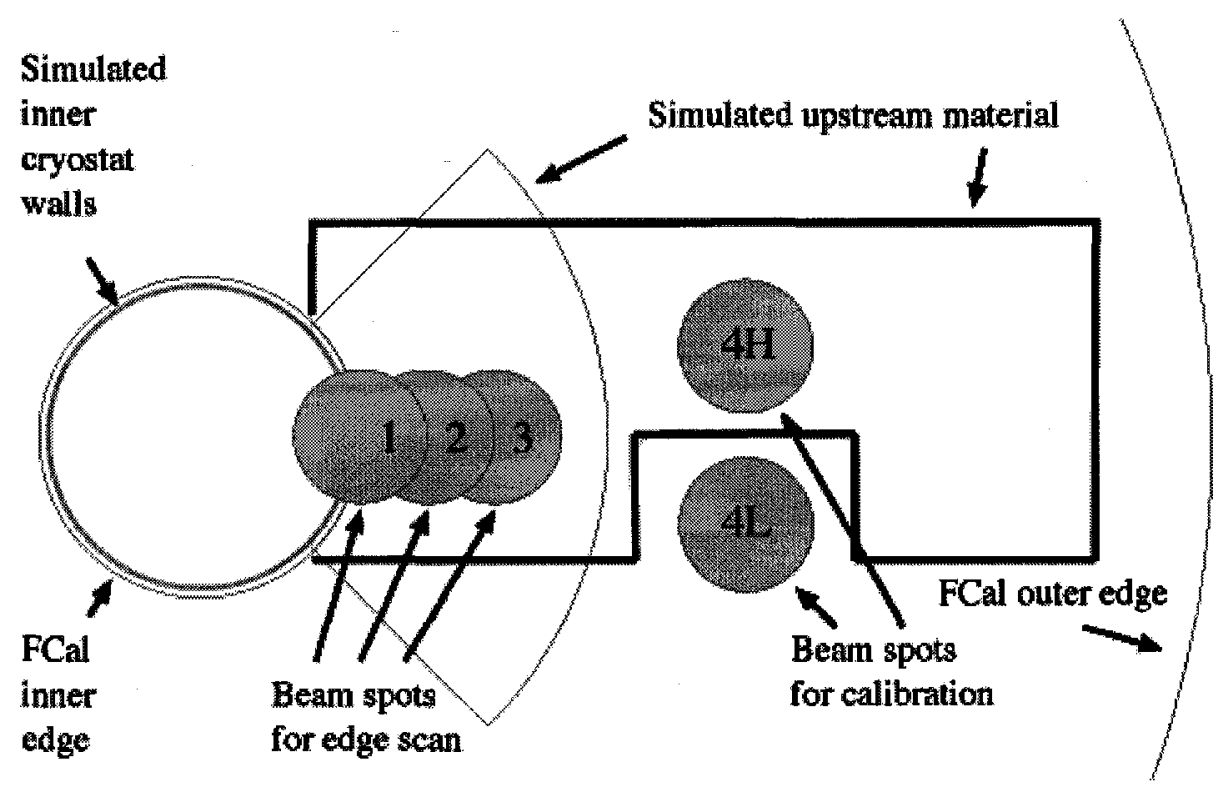

Figure 4.8: Schematic view of the FCal 2003 beam spots.

shown in Figure 4.3. Three additional positions were used to study inner detector effects and are referred to as positions 1,2 , and 3 . The cryostat was rotated and moved laterally to provide the five impact points and the angles that will be expected in the ATLAS setting. The angles used for the test beam are listed in Table 4.2.

\begin{tabular}{|c|c|c|}
\hline Beam Impact Point & Angle & $|\eta|$ \\
\hline \hline 1 & $0.88^{0}$ & 4.9 \\
\hline 2 & $1.25^{0}$ & 4.5 \\
\hline 3 & $1.61^{0}$ & 4.3 \\
\hline 4 & $2.98^{0}$ & 3.7 \\
\hline
\end{tabular}

Table 4.2: The beam impact points and angles used in the beam test along with the equivalent $|\eta|$ angle in ATLAS.

In the 2003 test beam, all channels were cabled up to the summing boards. However, due to the lack of pigtail cables ${ }^{3}$, only a subset of the channels were read out by the data acquisition system. The selection of instrumented read-out channels was made to optimize the measurement and containment of showers for the five impact points. The regions that

\footnotetext{
${ }^{3}$ Pigtail cables carry the signal from the summing board to the cryogenic signal feedthrough, as shown in Figure 4.9.
} 
were read out are shown in Figure 4.9.

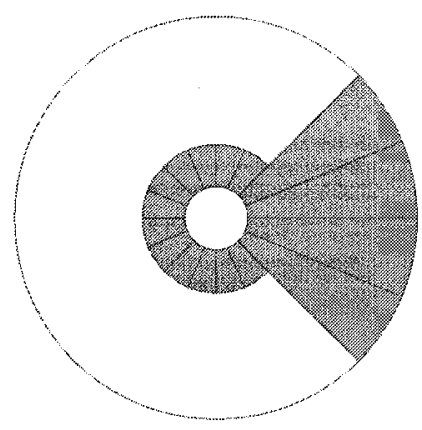

FCal1

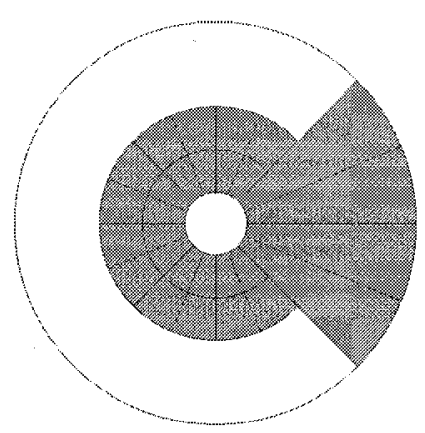

FCal2

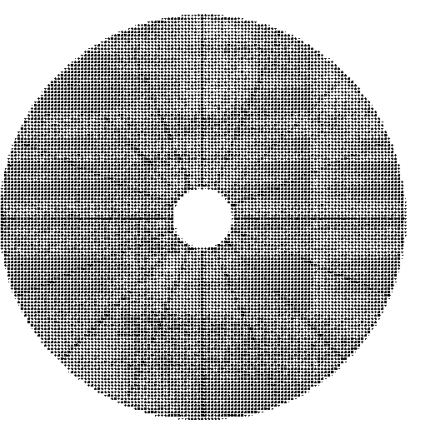

FCal3

Figure 4.9: Schematic diagram of the fully instrumented readout channels, shown in shaded areas. FCal 1 and FCal 2 emphasized the channels around the beam hole and on one side of the modules. FCal 3 was fully instrumented.

\subsection{Beamline Instrumentation}

To provide information about the particles in the beam, various detectors were used in the beam line. The data from these detectors was recorded along with the calorimeter data in order to provide offline beam quality control. In addition, some detectors were used to trigger the readout.

\section{Beam Positioning Chambers}

The Beam Positioning Chambers (BPCs) were used to measure the beam profile and to determine the beam impact position on the face of the FCal 1 module. There were three BPC stations, as illustrated in Figure 4.7, one located 1 meter downstream from the last bending magnet, the second was 11 meters downstream, and the final station was located 3 meters in front of the FCal modules. Each station consists of two $\mathrm{x}$-axis and $\mathrm{y}$-axis Multi Wire Proportional Chambers, providing a total of 6 measurements per axis. The position resolution for each measurement was approximately $200 \mu \mathrm{m}$, however, the resolution of the middle station was slightly worse $\sim 300 \mu \mathrm{m}$, owing to its older design. Beam tracks 
were reconstructed using the positions measured with these detectors.

$\mathrm{S} 1, \mathrm{S2}$, and S3

S1, S2, and S3 are $\sim 1 \mathrm{~cm}$ thick scintillators. Scintillators S1 and S2 have an area of $10 \times 10$ $\mathrm{cm}^{2}$, while S3 has a smaller area of $7 \times 7 \mathrm{~cm}^{2}$. These scintillators are located approximately 3 meters upstream from the FCal modules with S3 being the closest, followed by S2 and S1 (see Figure 4.7). These detectors were used in coincidence to indicate the passage of a beam particle and this coincidence signal was used to define a valid physics event. In addition, the $\mathrm{S} 1$ detector was used as the timing reference for the readout.

\section{Veto Hole}

The Veto Hole counter is a large scintillator with a $6.5 \mathrm{~cm}$ diameter hole centered on the beam line. This detector was used to remove events, at the analysis stage, in which the initial beam particle had scattered upstream resulting in a lower energy particle.

\section{Warm Tailcatcher}

The warm tailcatcher is located directly behind the cryostat that housed the FCal modules and was used to measure energy leakage and muon particles. It is composed of steel/scintillator parallel plates. The first three scintillator layers are $140 \times 140 \mathrm{~cm}^{2}$ followed by four $50 \times 50 \mathrm{~cm}^{2}$ scintillator layers.

\section{Muon Counter}

The muon counter is a $50 \times 50 \mathrm{~cm}^{2}$ scintillator placed behind a concrete beam stop directly behind the warm tailcatcher. The signal from the muon counter was used to indicate muon contamination.

\section{CEDAR}

The CEDAR is a high pressure gas Čerenkov device used for particle identification and 
was located in the beam line upstream of the last bending magnet. This device can be used to distinguish electrons and pions at energies below $\sim 100 \mathrm{GeV}$, and also to provide pion-proton separation. The CEDAR uses eight photo-tubes which provide three coincidence signals, $6 / 8,7 / 8$, and $8 / 8$. The results of these coincidence signals were written to the trigger word, event by event. It should be noted that only seven photo-tubes were functional during the test beam data taking periods. For the analysis in this thesis only the $6 / 8$ coincidence was used.

\subsection{Data Taking}

Data taking was recorded in three trigger modes: beam, random, and calibration. The beam trigger was used for the physics analysis and was defined using an online S1, S2, and S3 coincidence. This minimal beam trigger requirement was motivated by the desire to reduce any possible bias introduced by including additional beam line trigger elements. Random triggers were taken during and between beam spills when there were no beam particles and were used to study the electronic noise in the detectors. The calibration triggers were taken between the beam spills and were used to study the channel-to-channel response and to derive calibration pulse shapes.

There were two run types taken during the test beam: physics and calibration. For the physics runs, the modules were exposed to beams of electrons, pions, and muons within the energy range $5 \mathrm{GeV}<\mathrm{E}<200 \mathrm{GeV}$. Approximately 200,000 triggers were recorded for each electron energy per impact point and 400,000 events for each pion energy per impact point. The data in these runs were predominately taken in auto-gain mode with seven samples. Within a physics run, approximately $5 \%$ of the triggers were random triggers which were used for the pedestal ${ }^{4}$. In addition, dedicated calibration runs were taken when no test beams were present. These runs were used to study the behavior of the

\footnotetext{
${ }^{4}$ The pedestal is the value of the signal generated in a readout channel when no particle traverses the calorimeter.
} 
detector electronics such as the channel-to-channel gain variation. 


\section{Chapter 5}

\section{Data Treatment}

In this chapter, a study of the stability of the calorimeter system is described. This includes pedestal and noise studies. The pedestal and noise information was calculated and stored in a conditional database. Each data taking run has a set of pedestal and noise values for each readout channel. These values were calculated using the first sample from physics events within a physics test beam run. A run corresponds to 12,000 beam triggers $^{1}$ of which approximately $95 \%$ are physics events. This chapter provides a summary of the pedestal and noise stability analysis for runs between 1749 and 4315 .

\subsection{Pedestal Stability}

The pedestal stability was investigated for all of the physics runs. In order to determine the overall stability of the pedestal values, the average pedestal per run was calculated using all instrumented readout channels. This is referred to as the global pedestal and provides a measure of global pedestal problems or drifts. A linear fit was applied to the global pedestal value over runs 1749 to 4315 and this fit gave a slope of $(1.09 \pm 1.43) \times 10^{-5}$ and an intercept of $1016.74 \pm 0.04 \mathrm{ADCs}^{2}$. This shows that the global pedestal value was

\footnotetext{
${ }^{1}$ In the case when calorimeter data is collected for seven samples (time bins).

${ }^{2} \mathrm{ADC}$ units are the numerical values provided by the Analog to Digital Converter used to digitizing the data.
} 


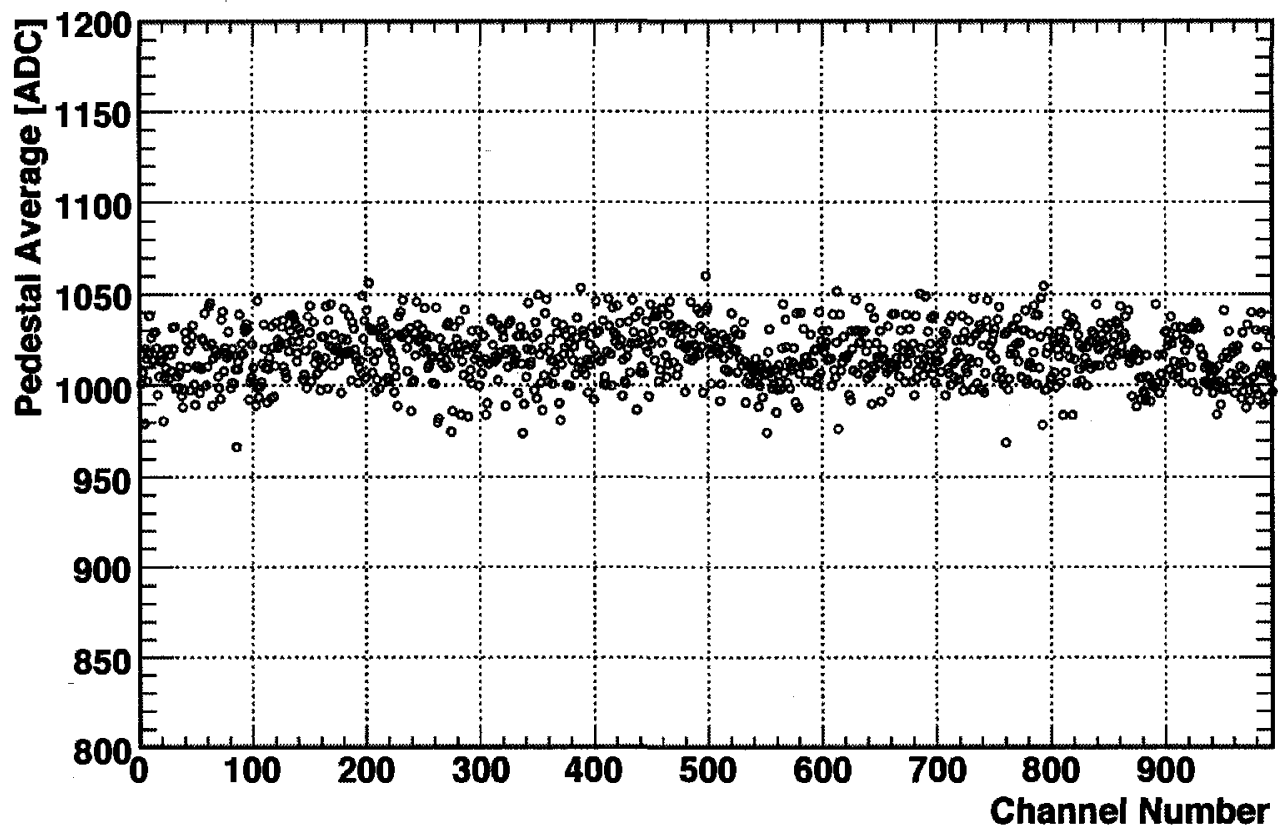

Figure 5.1: The average pedestal value as a function of channel number for runs between 1749 and 4315.

stable to approximately a hundredth of an ADC.

Next, the pedestal stability of each individual instrumented readout channel throughout runs 1749 to 4315 was investigated. The average value of the pedestal for each channel is shown in Figure 5.1. For each channel, the difference between the average pedestal across all runs and the average pedestal for a single run was calculated. The overall rms of these differences for all channels was calculated to be $(0.17 \pm 8.54) \times 10^{-5}$ ADC. This shows that the average pedestal across all runs and the average pedestal for a single run was stable.

\subsection{Noise Stability}

The magnitude and stability of the electronic noise for the global ${ }^{3}$ system and individual readout channels were investigated. To determine the overall noise stability of the calorimeter readout channels, the average noise for all channels as a function of the run

\footnotetext{
${ }^{3}$ We will refer to this as the global noise.
} 


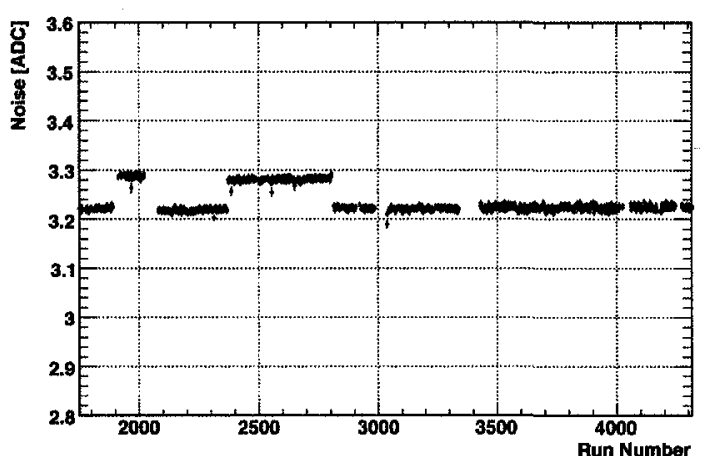

(a) Global noise value for every run between runs 1749 and 4315.

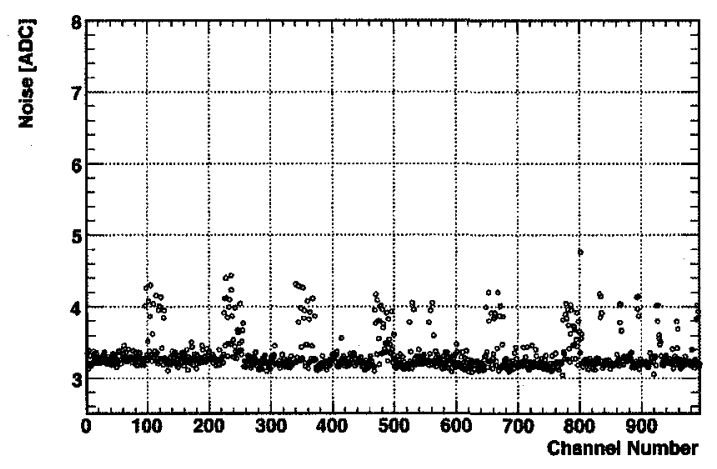

(b) Average individual channel noise for runs between runs 1749 and 4315 .

Figure 5.2: Results of noise studies illustrate instability in the electronic noise of the FCal readout channels, as shown in Figures 5.2(a) and 5.2(b).

number was calculated for the whole run period that was used for physics analysis. The result is shown in Figure 5.2(a) which clearly shows that the global noise was unstable, fluctuating during two regions of data taking. This was caused by a problem related to the calibration pulse system. This problem was noticed during data taking and corrected in later runs. The average noise for each individual readout cell for runs 1749 to 4315 was also calculated. These results are shown in Figure 5.2(b) and two subsets of the run period are shown in Figures 5.3(a) and 5.3(b). This clearly identifies problem channels, as well as the fact that these problem channels were only affected for some runs. The noise instability has a significant impact on the test beam analysis and this will be discussed in Section 7.3. 


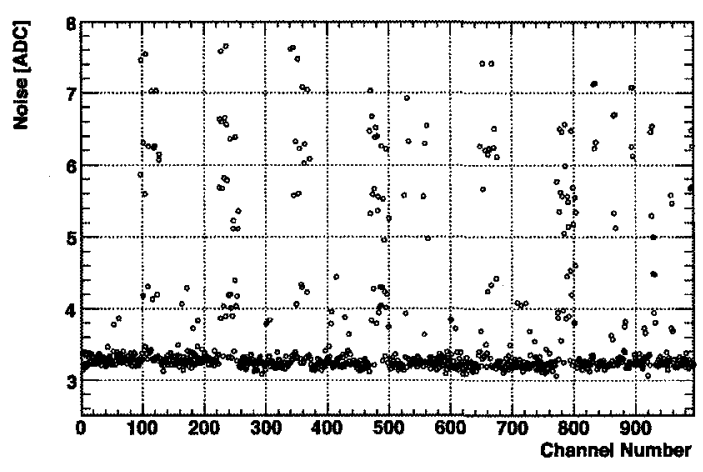

(a) Noise profile for runs between 2400 and 2800 .

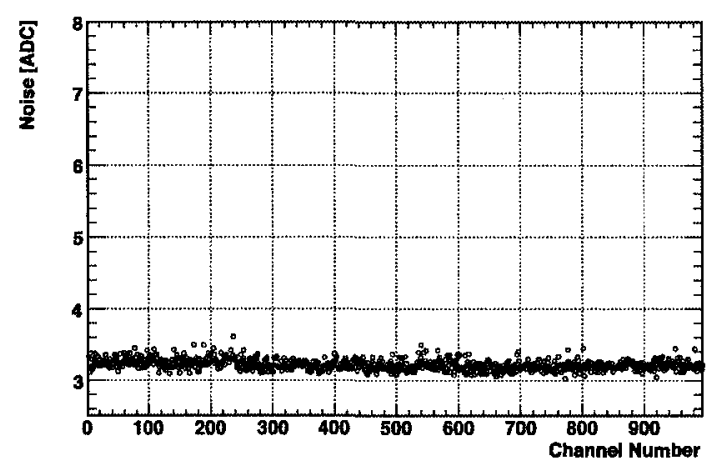

(b) Noise profile for runs between 3500 and 3900 .

Figure 5.3: Profile of the noise value for every run within specified range per channel for "noisy" runs 5.3(a) and "good" runs 5.3(b). 


\section{Chapter 6}

\section{Calorimeter Amplitude}

\section{Reconstruction}

\subsection{Introduction}

In this chapter, the procedure used to reconstruct the pulse height and timing information from the sampled data is described. In the 2003 test beam, readout channels were configured using auto-gain which allowed the appropriate gain to be selected based on a set threshold. All readout channels were predominately recorded in high gain and none in low gain. A few readout channels were recorded in medium gain, mainly in events with high energy electrons that resulted in a significant energy deposit in a single readout cell. This type of event would trigger the auto-gain feature to switch from high to medium gain.

The first step in the process to reconstruct the signal amplitude is to subtract the pedestals from the sampled data. As summarized in Chapter 5, pedestal values using the measurement of channels set to high gain were stored in a conditional database for each channel and run using the first time bin of physics events. However, for medium gain there was insufficient statistics in order to determine the pedestal from physics runs. Therefore, the 
pedestal values from dedicated dual gain random runs were used. The stability of these values were validated by comparing them to other dual gain pedestal runs.

Once the pedestal value was subtracted, a channel-to-channel gain correction was applied. These values were determined by setting the average high gain response for all the readout channels to 1 using calibration data. Therefore, each individual channel will have a channel-to-channel gain correction value relative to this normalization. Similarly, the medium channel-to-channel gain correction was determined relative to the high gain response. Therefore, once all these channel by channel calibration factors have been applied, all readout channels are calibrated to high gain.

Readout channels located on the inner and outer edges of the detector must be corrected for the gain difference that arises due to the fact that they are not summed on the summing board. Although this correction does not affect the analysis in this thesis due to the beam position studied, this correction will be very important for the inner edge studies and for ATLAS running. Once all the above corrections have been applied, the samples in a readout channel can be used to reconstruct the peak amplitude of the signal pulse by applying Optimal Filtering Coefficients (OFCs). The optimal filtering (OF) technique [24] is the pulse reconstruction technique chosen by the Liquid Argon Community. The remainder of this chapter is dedicated to the determination and application of the OFCs and all associated requirements.

\subsection{Test Beam Event Phase}

In the 2003 test beam, the beam trigger and the sampling clock, Trigger Timing Control (TTC) $40 \mathrm{MHz}$ clock, are asynchronous. However, in order to apply the Optimal Filtering Technique the event phase must be known to better than a few nanoseconds. Therefore, the time phase between the TTC clock and the beam trigger needs to be calculated for 
each event. This quantity is referred to as the relative phase and is determined by starting a TDC clock using the $S 1$ trigger and stopping it using the sampling clock. In addition, a second TDC clock delayed by approximately $10 \mathrm{~ns}$ with respect to the original TDC clock was used to prevent ambiguous timing values when a TDC clock is in phase with the sampling clock. Using the TDC clock time, the phase of an event can be calculated using Equation 6.1.

$$
t_{p h a s e}=\alpha\left(t_{t d c}-w a c\right)
$$

Here, $\alpha$ is the value which converts the TDC measurement, $t_{t d c}$, to nanoseconds (ns) and $w a c$ is the wrap-around-constant. The $\alpha$ value is calculated by dividing the sampling interval, that is $25 \mathrm{~ns}$, by the range of the TDC measurement. Each TDC clock measurement produces a $t_{\text {phase }}$ with its own $\alpha$ and wac values. In addition, a phase quality was calculated for each phase measurement. These phase qualities were used to determine the best phase value for a given event and to remove events for which both phase values were problematic. There were three regions of concern for the reconstructed event phase. These were for $t_{\text {phase }}$ values near the minimum or maximum of the $t_{t d c}$ range or $t_{\text {phase }}$ values that were close to wac. The phase quality was calculated by selecting the smaller of the differences between the TDC value and the problematic TDC values. Of the two phases, the one with the largest phase quality was used as the event phase.

\subsection{Optimal Filtering Technique}

The optimal filtering technique is the linear application of coefficients to a sampled pulse shape in order to determine the peak and time delay of the pulse. The time delay is the timing difference between the reference pulse shape and the sampled pulse shape. Ideally the time delay is zero if the OFCs are properly calibrated. However, it can be non zero if the reference pulse shape has been derived with an arbitrary start time. The calibration procedure is summarized in Section 6.3.3. Presented in this section is a brief summary of the optimal filtering technique. 
The signal at time, $t$, can be approximated using Equation 6.2.

$$
S_{t}=A \cdot p(t-\tau)+n(t)
$$

Here, $A$ is the amplitude and $p(t-\tau)$ is the normalized pulse shape with a time delay, $\tau$. The $n(t)$ function represents the sum of the noise contributions from the electronics chain. If the time delay is small, the signal can be approximated using Equation 6.3.

$$
S_{t}=A\left[p(t)-\tau p^{\prime}(t)\right]+n(t)
$$

Here, $p^{\prime}(t)$ is the derivative of the pulse shape. The ATLAS calorimeter system samples the pulse shape every $25 \mathrm{~ns}$. Therefore, the signal amplitude for the $\mathrm{i}^{\text {th }}$ sample of the pulse shape at time $t_{i}$ is given by Equation 6.4.

$$
S_{i}=A\left[p\left(t_{i}\right)-\tau p^{\prime}\left(t_{i}\right)\right]+n_{i}
$$

Next, two variables, $u$ and $v$, as defined in Equation 6.5, are used to reconstruct the peak and time delay values of the pulse.

$$
u=\sum_{i} a_{i} S_{i} \quad v=\sum_{i} b_{i} S_{i}
$$

In Equation 6.5, variables $a$ and $b$ are the OFCs. The derivations of these coefficients are summarized below.

As stated above, the goal of the optimal filtering technique is to determine the peak position and the time delay of the pulse shape. In order to reconstruct the desired energy and 
time delay, the mean of $u$ and $v$ are constrained as shown in Equations 6.6 and 6.7 .

$$
\begin{gathered}
<u>=A \quad \Rightarrow \sum_{i=1} a_{i} p\left(t_{i}\right)=1 ; \quad \sum_{i=1} a_{i} p^{\prime}\left(t_{i}\right)=0 \\
<v>=A \tau \quad \Rightarrow \sum_{i=1} b_{i} p\left(t_{i}\right)=0 ; \quad \sum_{i=1} a_{i} p^{\prime}\left(t_{i}\right)=-1
\end{gathered}
$$

Next, using Equations 6.5 and 6.4, the variance of $u$ and $v$ can be determined and are shown in Equations 6.8 and 6.9.

$$
\begin{aligned}
& \sigma(u)^{2}=\sum_{i=1} \sum_{j=1} a_{i} a_{j} R_{i j} \\
& \sigma(v)^{2}=\sum_{i=1} \sum_{j=1} b_{i} b_{j} R_{i j}
\end{aligned}
$$

Here $R_{i j}$ is the noise auto-correlation matrix and will be discussed in Section 6.3.1. By minimizing $\sigma(u)^{2}$ and $\sigma(v)^{2}$ while constraining the means, a set of OFCs can be determined. This prescription provides the optimal energy and time resolution while constraining the mean values of $u$ and $v$ to the desired values, i.e. the correct amplitude and time delay. In the above derivations, the average noise is assumed to have a zero mean value, $\langle n(t)\rangle=0$. However, the correlation between the noise in neighboring samples, $<n_{i} n_{j}>=R_{i j}$, is assumed to be non-zero.

It is clear from the above description that a good understanding of the noise auto-correlation, the pulse shape, and the derivative of the pulse shape is required. Therefore, a summary of how the auto-correlation and pulse shape are determined is presented in Sections 6.3.1 and 6.3 .2 , respectively. 


\subsubsection{Auto-Correlation Matrix}

The correlation between two time samples $s_{i}$ and $s_{j}$ is defined in Equation 6.10.

$$
R_{i j}=\frac{\operatorname{cov}\left(s_{i}, s_{j}\right)}{\sigma\left(s_{i}\right) \sigma\left(s_{j}\right)}
$$

From these correlations, an $i \times j$ matrix can be established. The diagonal terms of the matrix were averaged to define the auto-correlation matrix. The auto-correlation matrix was calculated using pedestal events and stored in the database for every run.

\subsubsection{Pulse Shape Analysis}

Presented in this section are the results from analysis performed to determine the pulse shape for the 2003 test beam. The pulse shape for all channels within a given module are approximately the same because of the uniformity of the mechanical construction of the modules. Therefore, only one pulse shape for each FCal module was required.

In the 2003 test beam, two separate physics pulse shape analysis techniques were performed. The first, a standalone program, relied on prior knowledge of the physics pulse shape derived using a SPICE $^{1}$ model [25]. The second, which will be discussed in detail in this thesis was developed within the Athena framework and did not require prior knowledge of the physics pulse shape but instead relied on the $t_{\text {phase }}$ analysis discussed in Section 6.2. The $t_{\text {phase }}$ was used to provide a consistent time reference frame from event-to-event and to accumulate individual channel pulse shapes over a large number of events. A limitation in the Athena analysis was the time resolution which was increased by binning effects and rare but problematic phase shifts.

For the data used in the Athena based analysis, the pulse shape was sampled using beam

\footnotetext{
${ }^{1}$ SPICE is a software tool which simulates and analyses electronic circuits ranging from simple single resistor circuits to sophisticated circuits comprising tens-of-thousands of components.
} 
data every $25 \mathrm{~ns}$ with a total of seven samples. The first sample was used to determine the pedestal (internal offset) and the signal peak was typically located in the fourth sample. Because the beam time was asynchronous to the sampling clock, the peak of the pulse shape was randomly distributed with respect to the $25 \mathrm{~ns}$ sampling window and an event phase was required (see Section 6.2).

Readout channels with a peak sample greater than 5 times the electronic noise for the channel were selected for analysis. The pulse shape was determined by accumulating the samples for a large number of events. Once all the events had been processed, the raw pulse shape was normalized by the maximum pulse height, which was determined using a spline fit to the raw pulse shape. Next, pulse shapes were created for each module by adding all the channels in a given module. This provided a first approximation to the true pulse shape. In the last step, each channel was re-processed to correct for any minor time shift by taking the time difference at the peak amplitude between the channel and module pulse shapes.

To estimate the error on the physics pulse shape, the pulse shape was investigated at four different beam impact positions. The data used for this analysis is summarized in Table 6.1. In addition, a global pulse shape was determined using the data from all four positions. The error on the global pulse shape was estimated based on its maximum variation from the pulse shapes at the four impact points.

Finally, the global pulse shape was compared to the pulse shape derived from the stan-

\begin{tabular}{|c|c|c|}
\hline Run Number & Position & Number of Events \\
\hline 1852 to 1867 & 1 & 183020 \\
1923 to 1938 & 2 & 182166 \\
1749 to 1764 & $4 \mathrm{~L}$ & 184804 \\
2083 to 2098 & $4 \mathrm{H}$ & 178516 \\
\hline
\end{tabular}

Table 6.1: Physics pulse shape analysis data summary. 
dalone analysis using a $\chi^{2}$ goodness-of-fit test. The results of the $\chi^{2}$ goodness-of-fit tests are shown in Table 6.2. Therefore, at a 95\% confidence level the Athena and the standalone pulse shapes are in agreement within the assumed errors. Figure 6.1 show the results of the standalone and the Athena based results for the FCal 1 module. Similar comparisons were preformed for the FCal 2 and FCal 3 modules. The two methods were deemed to be equivalent and based on a group policy decision it was decided to use the pulse shape derived from the standalone analysis. For consistency, this method has also been used in the analysis for this thesis.

\begin{tabular}{|c|c|c|}
\hline Module & $\chi^{2} / \nu$ & $\chi_{95 \%}^{2}$ \\
\hline FCal1 & $77.6847 / 150$ & 179.581 \\
FCal2 & $135.465 / 151$ & 180.676 \\
FCal3 & $34.944 / 151$ & 180.676 \\
\hline
\end{tabular}

Table 6.2: Physics pulse shape $\chi^{2}$ goodness-of-fit summary from the analysis comparing the two pulse shape analysis methods. Here $\nu$ is the number of degrees of freedom and $\chi_{95 \%}^{2}$ is the $\chi^{2}$ function for $\nu$ degrees of freedom at a $95 \%$ confidence level.

\subsubsection{Calibration and Validation of the Optimal Filtering Coefficients}

The pedestal-subtracted and gain corrected signals were processed by applying the optimal filtering technique using time samples 2 through 6 of the seven samples. The OFCs were determined using the physics pulse shape and the autocorrelation matrix as discussed in Sections 6.3.2 and 6.3.1 respectively. The OFCs were calculated with one nanosecond binning $^{2}$ in order to account for the asynchronous beam trigger and the sampling clock times. As indicated in the previous section, the pulse shapes used to determine the OFCs were derived from a standalone analysis. As a consequence, the OFCs needed to be calibrated such that their reference times were consistent with the event phase defined in Section 6.2. The timing calibration was iterative, starting with an initial set of OFCs determined without any calibration. Afterwards, the un-calibrated OFCs were applied to the data and the reconstructed time delays were calculated. These time delays were used to align the

\footnotetext{
${ }^{2}$ Limited by the NOVA conditional database data structure at the time of the implementation.
} 


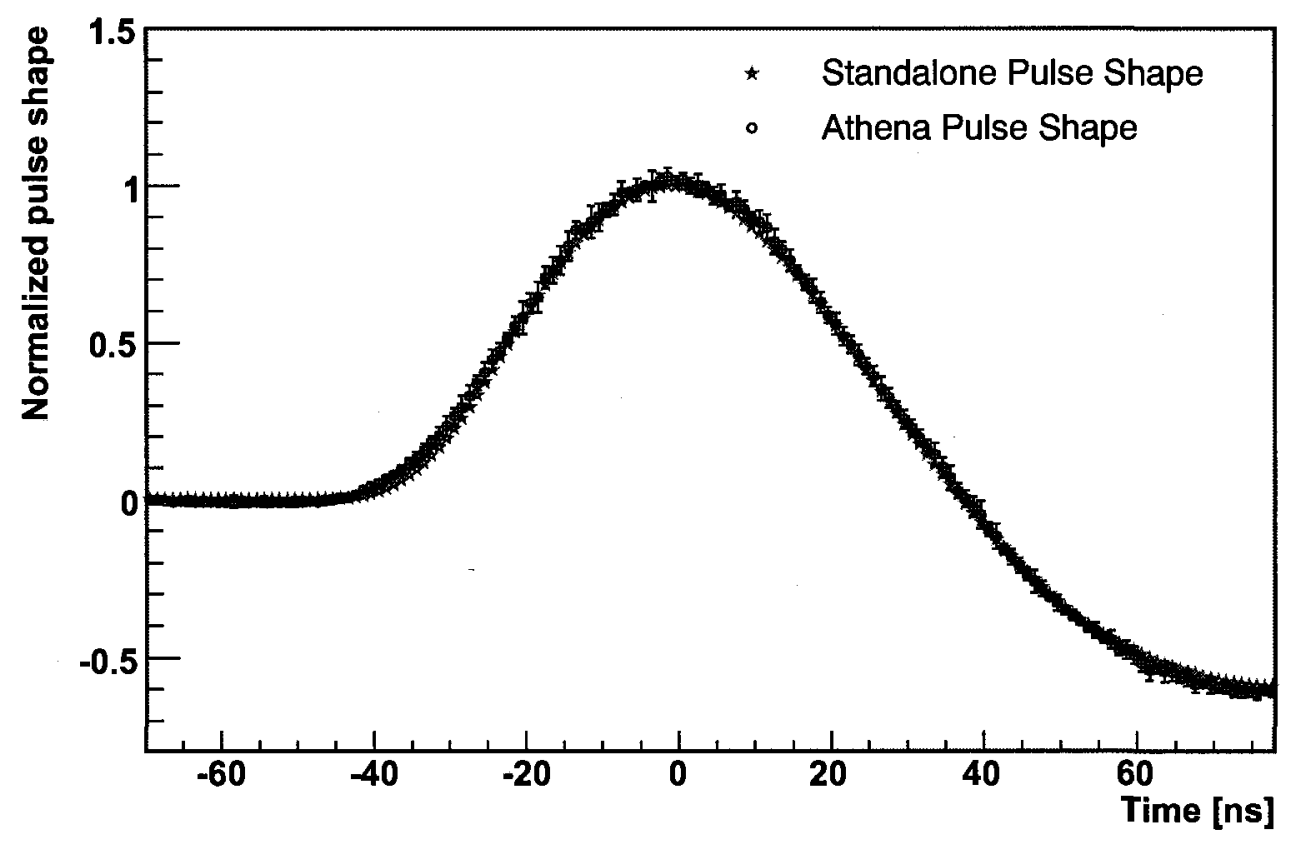

Figure 6.1: Results of the standalone (labelled by stars) and Athena (labelled by circles) pulse shape analyses for the FCal 1 module. The error bars are for the Athena analysis and were estimated based on its maximum variation between the global pulse shape and the pulse shapes at different beam spots. The pulse shapes are in good agreement within the assumed errors. Similar comparisons were preformed for the FCal 2 and FCal 3 modules. 
standalone pulse shape reference time to the Athena event phase. As such, a set of timing calibration values were determined using the OFC time delays for each readout channel in each gain mode. In addition, an average calibration time for each Front-End Board (FEB) was calculated. These FEB time calibration values were used for channels without sufficient energy to properly reconstruct the time. From these new time calibration values, a new set of OFCs were determined and the cycle was repeated until the time delay was centered at zero. The OFC calibration technique converges very quickly, as shown in Figure 6.2. In Figure 6.2(a), the un-calibrated OFC time delays for the channels in the FCal

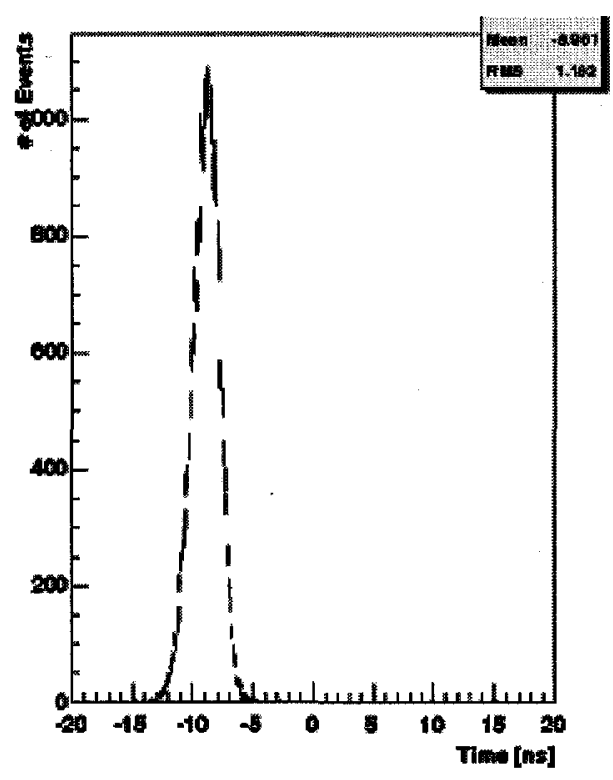

(a) Reconstructed OFC time delay without calibration iterations. The mean and RMS for this OFC time delay, $\tau$, was -8.9 ns and $1.192 \mathrm{~ns}$, respectively.

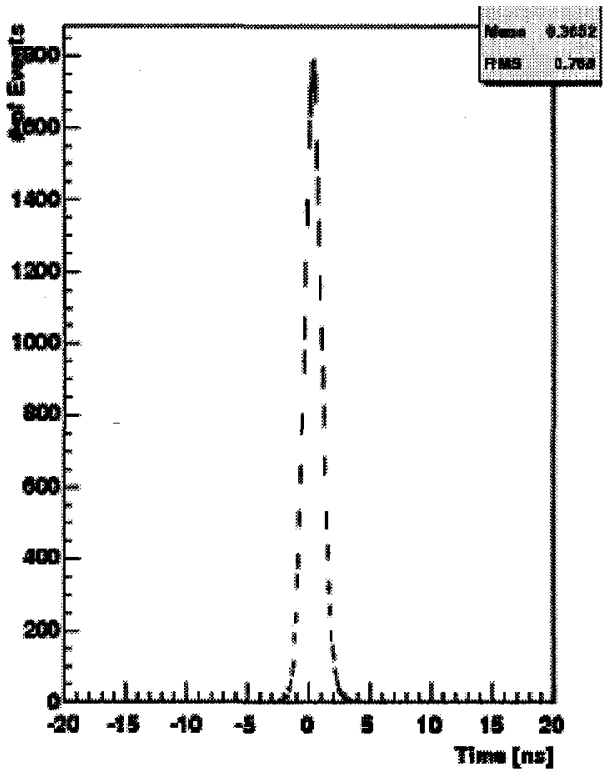

(b) Reconstructed OFC time delay after two calibration iterations. The mean and RMS for this OFC time delay, $\tau$, was 0.37 ns and $0.77 \mathrm{~ns}$, respectively.

Figure 6.2: Calibration of the OFC time for the FCal 2 module determined using $200 \mathrm{GeV}$ pion beam data. The timing resolution was reduced by $\sim 65 \%$ within two iterations.

2 module were centered at $\mathbf{- 8 . 9}$ ns. Within two iterations, these time delays were corrected to $0.37 \mathrm{~ns}$, as shown in Figure 6.2(b). In addition, the OFC time delay resolution for the FCal 2 module was reduced by $\sim 65 \%$.

In order to validate the OFCs, it is necessary to verify that the peak of the OFC normal- 
ized pulse shapes is one. This provides confidence that the applied OFCs will reconstruct the amplitude correctly. In addition, the normalized OFC pulse shape is compared to the original pulse shape used to derive the coefficients.

The OFC normalized pulse shapes for each FCal was determined by accumulating the pulse shape for each readout channel in a given module over a large number of events. Readout channels with a peak sample greater than five times its electronic noise were used for this analysis. For each event, the sample for each readout channel was normalized using the reconstructed OFC amplitude, as shown in Equation 6.11

$$
N_{i}\left(t_{\text {phase }}\right)=\frac{S_{i}}{\sum_{i=0}^{n} S_{i} a_{i}\left(t_{\text {phase }}\right)}
$$

Here, $N_{i}\left(t_{\text {phase }}\right)$ is the normalized $i^{t h}$ sample for a given readout channel corresponding to the $t_{\text {phase }}$ phase delay, $S_{i}$ is the raw $i^{\text {th }}$ sample value, and $a_{i}\left(t_{\text {phase }}\right)$ is the OFC for the $i^{\text {th }}$ sample corresponding to the $t_{\text {phase }}$ phase delay. Since the OFCs rely on the event time phase, a cut on the events with an event phase quality greater than 15 TDC units was applied to avoid poorly reconstructed event phases.

The peak of the OFC normalized pulse shape was found to be within $1 \%$ of unity for all three FCal modules, verifying the OFC technique (see Figure 6.3). In addition, comparing the OFC normalized pulse shape in Figure 6.3 with the original pulse shape in Figure 6.1 gives good agreement. Figures 6.3(a) to 6.3(c) show the reconstructed OFC normalized pulse shapes. 

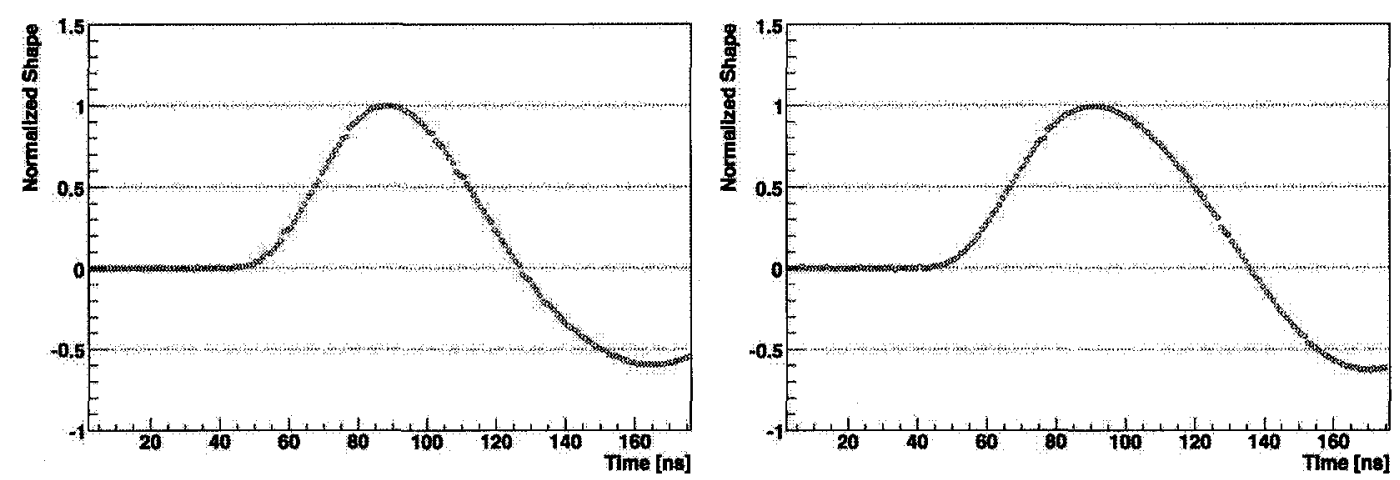

(a) Normalized OFC pulse shape for FCal 1 module.

(b) Normalized OFC pulse shape for FCal 2 module.

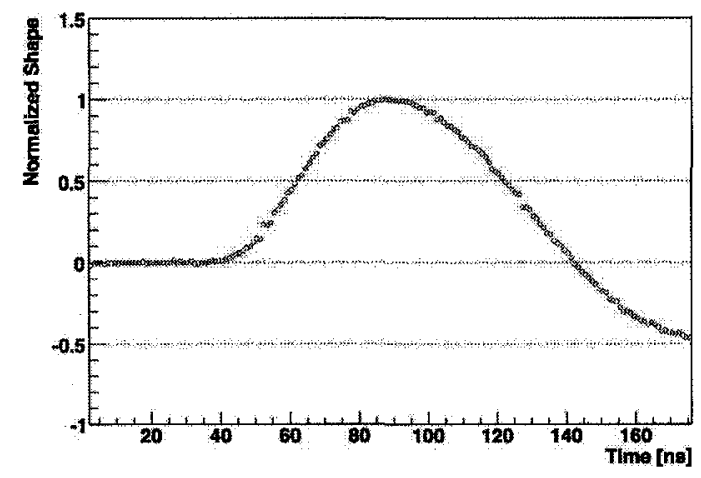

(c) Normalized OFC pulse shape for FCal 3 module.

Figure 6.3: Results of the OFC normalized pulse shape. For all three modules the peak amplitude is within $1 \%$ of unity. 


\section{Chapter 7}

\section{Test Beam Results}

This chapter presents the FCal performance results for electrons and hadrons impacting the $4 \mathrm{~L}$ position. Beam cleaning and envelope cuts are applied to the data of both beam types as explained in Section 7.1. A review of the use of the beam track information for clustering and hadronic weighting is presented in Section 7.2. The clustering technique and its application to noise correction is presented in Section 7.3. Finally, the results from the electron and hadron analyses are presented in Sections 7.4 and 7.5.

\subsection{Beam Cleaning and Envelope Cuts}

Preliminary beam cleaning was carried out using the information in the offline trigger. As such, events were rejected if multiple scattering occurred or-when events were too close in time. In addition, information from the CEDAR was used for both the electron and hadron analyses and its use will be summarized for each analysis.

A more sophisticated beam cleaning procedure was developed using information from the various beam line scintillators to remove events in which particles had scattered up stream and events with multiple beam particles. This was accomplished by studying the scintillators characteristics, such as pedestal, pedestal width, and single-particle response 
as a function of the run number. Once the response of the scintillators was well understood, various cuts were applied on combinations of signals. Using such combinations, as opposed to cutting on a defined threshold for each channel, avoids rejecting valid events due to one noisy individual channel. As an example, it is clear that cutting on S1 or S2 at

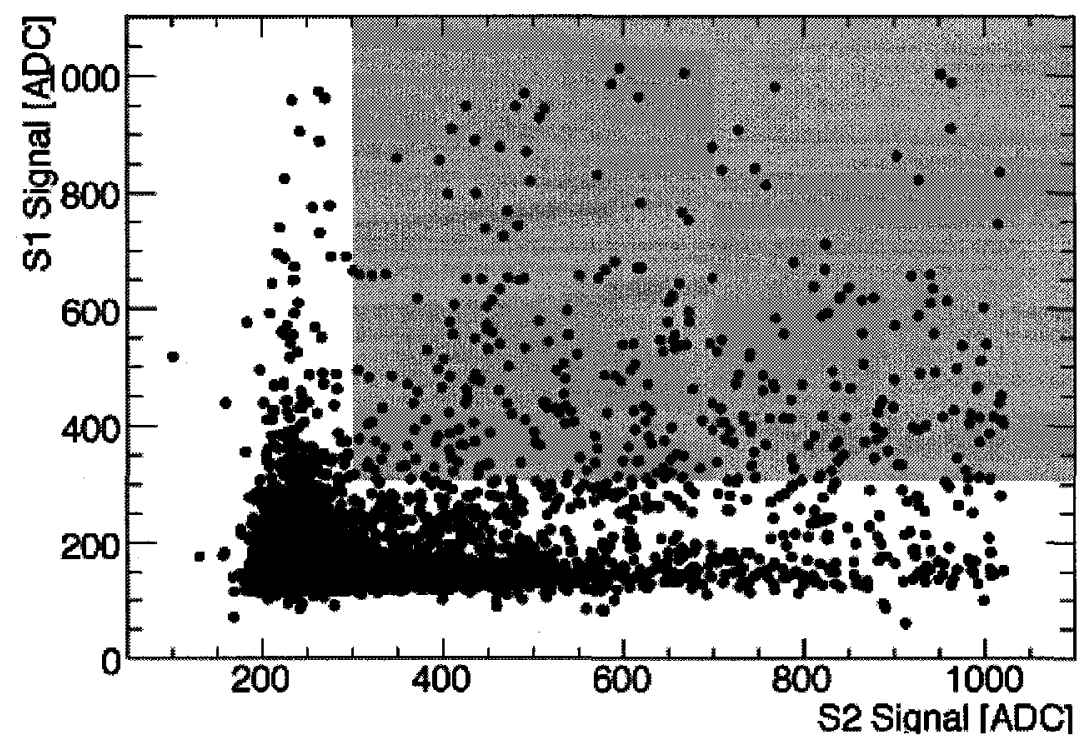

Figure 7.1: Example of S1 and S2 combination information. It is clear that cutting on S1 or $\mathrm{S} 2$ individually would remove potentially valid events. Therefore, a cut on the combined signal of S1 and S2 is used to remove events that fall in the shaded area.

300 ADC counts individually would remove potentially valid events. Therefore, a cut on the combined signal of S1 and S2 is used, as shown in Figure 7.1.

In addition, a more sophisticated "beam envelope cut" was applied to differentiate electrons from hadrons. This was based on using the impact position of the beam particles on the calorimeter face and the slope of their trajectories. Track information was provided by the reconstructed tracks using the six Beam Position Chambers (BPC). 


\section{2 $\quad$ Tracking}

The tracking system, which consists of six BPCs, was used to determine the precise location of the beam impact point on the face of the FCal modules and to define the beam trajectory. The need to determine a precise impact position is primarily motivated by the transverse weighting technique used to achieve compensation when analyzing the hadron data. This technique requires good track information and will be discussed in Section 7.5.3. As mentioned in Section 7.1, the beam trajectory was also used to separate electrons and hadrons.

In order to use the tracking system, the reconstructed tracks must be aligned to the FCal coordinate system. The first step in this alignment procedure requires mapping the position of the reconstructed tracks to the calculated center-of-gravity of the showers in the FCal 1 module. However, some calorimeter readout channels had excessive noise in some runs, as discussed in Section 5.2, which affected the center-of-gravity calculation. Therefore, the stability of the center-of-gravity calculation is investigated in Section 7.2.1 using various energy cuts. In addition, the center-of-gravity calculation is biased by the "pulling" of the geometric center from the most energetic readout channel. Therefore, a second step is used to remove this bias and improve the determination of the beam impact point as presented in Section 7.2.2.

\subsubsection{Calorimeter Based Transverse Shower Position Calculation}

In this section, the stability of the center-of-gravity calculation for the transverse shower position as a function of the cell signal significance (signal/noise) was investigated. For this study, the center-of-gravity is calculated using only the signals from the readout channels for FCal 1. The same center-of-gravity calculation was used for both $\mathrm{x}$ and $\mathrm{y}$ coordi- 
nates and is shown in Equation 7.1 for the $\mathrm{x}$-axis.

$$
x_{c g}=\frac{\sum\left(x_{\text {cell }} \cdot E_{\text {cell }}\right)}{\sum E_{\text {cell }}}
$$

Here, $x_{c g}\left(y_{c g}\right)$ is the center-of-gravity of the shower, $x_{c e l l}\left(y_{c e l l}\right)$ is the $\mathrm{x}(\mathrm{y})$ position of the readout cell ${ }^{1}$, and $E_{\text {cell }}$ is the reconstructed energy of the cell.

The signal significance is defined as the ratio between the cell energy (E) and its electronic noise $(\sigma)$. The signal significance was considered to avoid the reconstructed center-ofgravity being "pulled" by noisy cells. Cells with a signal significance, $E / \sigma_{\text {noise }}$, above a significance threshold, $\mathcal{S}$, were included in the center-of-gravity calculation. In addition, the effect of the reconstructed center-of-gravity using cells with $E / \sigma_{\text {noise }}>\mathcal{S}$ or $\left|E / \sigma_{\text {noise }}\right|>\mathcal{S}$ was studied.

For this analysis, runs corresponding to a $100 \mathrm{GeV}$ electron at the $4 \mathrm{~L}$ beam position were used. From the results shown in Table 7.1, the center-of-gravity determination stabilizes at a signal significance of 3-4. Moreover, the absolute significance cut, $\left|E / \sigma_{\text {noise }}\right|>\mathcal{S}$, is preferred since the absolute cut treats the noise symmetrically. Therefore, a $\left|E / \sigma_{\text {noise }}\right|>3$ is preferred when calculating the transverse shower position.

\begin{tabular}{|c|c|c|}
\hline $\begin{array}{c}\text { Significance cut } \\
(\mathrm{E} / \sigma)\end{array}$ & $\begin{array}{c}\text { Mean Position } \\
(\mathrm{x}, \mathrm{y})[\mathrm{mm}]\end{array}$ & $\begin{array}{c}\text { Mean Position with } \\
\text { Absolute Cut }(\mathrm{x}, \mathrm{y})[\mathrm{mm}]\end{array}$ \\
\hline \hline 0 & $219.0,-35.85$ & $242.2,-47.00$ \\
1 & $229.4,-39.73$ & $242.1,-47.15$ \\
2 & $241.2,-45.56$ & $243.9,-47.48$ \\
3 & $245.9,-47.92$ & $246.3,-47.98$ \\
4 & $246.3,-48.20$ & $246.5,-48.22$ \\
5 & $246.3,-48.25$ & $246.3,-48.26$ \\
\hline
\end{tabular}

Table 7.1: Effect of significance cuts on the mean center-of-gravity position for $100 \mathrm{GeV}$ electron shower at the $4 \mathrm{~L}$ position.

\footnotetext{
${ }^{1}$ The position of the cell is defined as the average position of the electrodes within the cell.
} 


\subsubsection{Tracking and Calorimeter Alignment}

To improve the determination of the shower center, tracking information from the BPCs was used. The beam tracking coordinate system must be defined by aligning each individual BPC coordinate system to a common coordinate system. This was achieved by aligning all the BPCs to the BPC closest to the last bending magnet, referred to as BPC 1. Afterwards, a linear fit in both geometrical planes ( $x-z$ and $y-z)$ was performed. The residual between the linear fit and the individual BPC coordinate system was used to align each BPC to the defined tracking coordinate system, that is BPC 1 .

Next, the reconstructed tracks were aligned to the FCal 1 coordinate system. This was achieved in two steps. In the first step, a crude alignment shift was calculated for both $\mathrm{x}$ and y using the center-of-gravity calculation for the FCal 1 modules. These crude shifts were determined using a $\chi^{2}$ minimization ${ }^{2}$ in both the $\mathrm{x}$ and $\mathrm{y}$ directions, as shown in Equation 7.2 for the $\mathrm{x}$ direction.

$$
\chi^{2}=\left(x_{\text {track }}-x_{\text {calo }}-x_{\text {shift }}\right)^{2}
$$

Here, $x_{\text {track }}\left(y_{\text {track }}\right)$ is the track position in the beam line coordinate system, $x_{\text {calo }}\left(y_{\text {calo }}\right)$ is the center-of-gravity position in the calorimeter coordinate system as explained in Section 7.2.1, and $x_{\text {shift }}\left(y_{\text {shift }}\right)$ is the relative shift needed to correct for the difference between the two coordinate systems. The results of the first step are presented in Table 7.2. In a similar manner to the center-of-gravity calculation, the track shift converges using a significance threshold of 3 or greater.

The second step was performed to correct for the "pulling" of the geometrical cell center when calculating the center-of-gravity. This was achieved by scanning the boundaries between readout cells and looking at the variation of the ratio $E_{1, \max } / E_{1}$ using the align-

\footnotetext{
${ }^{2}$ The error is assumed to be equal for all events used in the minimization and is omitted.
} 


\begin{tabular}{|c|c|c|}
\hline $\begin{array}{c}\text { Significance cut } \\
(\mathrm{E} / \sigma)\end{array}$ & $\begin{array}{c}\text { Track Shift } \\
(\mathrm{x}, \mathrm{y})[\mathrm{mm}]\end{array}$ & $\begin{array}{c}\text { Track Shift with } \\
\text { Absolute Cut }(\mathrm{x}, \mathrm{y})[\mathrm{mm}]\end{array}$ \\
\hline \hline 0 & $222.33,15.1$ & $243.07,4.48$ \\
1 & $232.72,11.3$ & $243.91,4.15$ \\
2 & $244.99,6.06$ & $250.01,4.67$ \\
3 & $250.35,3.50$ & $250.70,3.36$ \\
4 & $251.48,2.71$ & $251.49,2.69$ \\
5 & $251.50,2.64$ & $251.49,2.64$ \\
\hline
\end{tabular}

Table 7.2: Effect of significance cuts on track shifts for $100 \mathrm{GeV}$ electron beam at the $4 \mathrm{~L}$ position.

ment corrections for the $\mathrm{x}$ and $\mathrm{y}$ directions from the first step. Here, $E_{1, \max }$ is the highest energy cell in FCal 1 and $E_{1}$ is the total energy in FCal 1. The minimum of the distribution in $\mathrm{x}$ and $\mathrm{y}$ should coincide with the average position between electrodes in neighboring readout cells. Therefore, if the tracks are properly aligned, the minimum should match the FCal 1 electrode pattern.

For this analysis, track shifts obtained using the absolute significance cut of 5 in Table 7.2 were used. The local minimum using the corrections in the first step for the $\mathrm{x}$ direction was determined at $1.76 \mathrm{~mm}$, as shown in Figure $7.2(\mathrm{a})$. Similarly, the minimum for the track scan in the y direction using the corrections in the first set was determined to be 1.57 $\mathrm{mm}$, as shown in Figure 7.2(b). These minima were used to correct the tracking alignment found in the first step. Therefore, the new track alignment shifts are $249.73 \mathrm{~mm}$ and 1.07 $\mathrm{mm}$ for the $\mathrm{x}$ and $\mathrm{y}$ direction, respectively. Figure 7.3 shows the new $E_{1, \max } / E_{1}$ with the additional correction to the alignment shifts. It should be noted that the slight asymmetry in the $\mathrm{x}$ distribution in Figure 7.3(a) is assumed to be due to the beam impact angle.

\subsubsection{Stability of Corrected Tracking Shifts}

The stability of the track shifts for electrons in position $4 \mathrm{~L}$ was investigated for the runs shown in Table 7.3. The technique presented in the previous section requires that a large fraction of the total shower energy, $\sim 50 \%$, be contained within a single readout cell in 


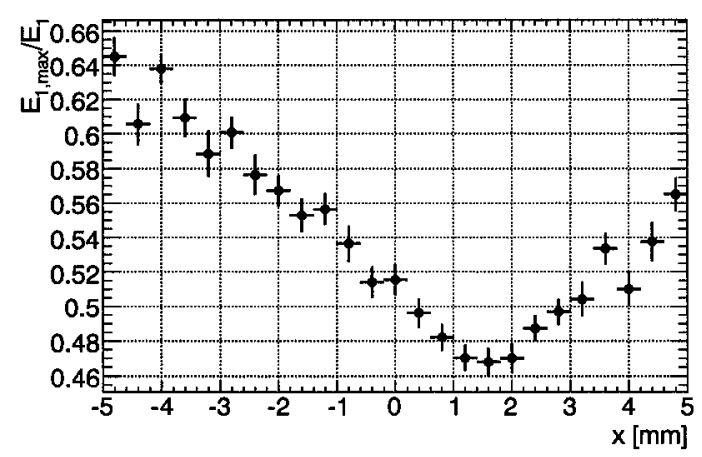

(a) Track scan in $\mathrm{x}$ of $E_{1, \max } / E_{1}$

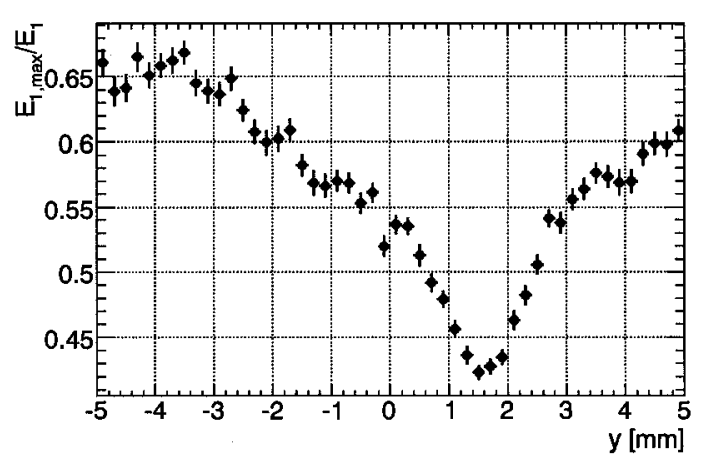

(b) Track scan in y of $E_{1, \max } / E_{1}$

Figure 7.2: Plots of $E_{1, \max } / E_{1}$ relative to $\mathrm{x}$ and $\mathrm{y}$ using the shift corrections for the centerof-gravity calculation.

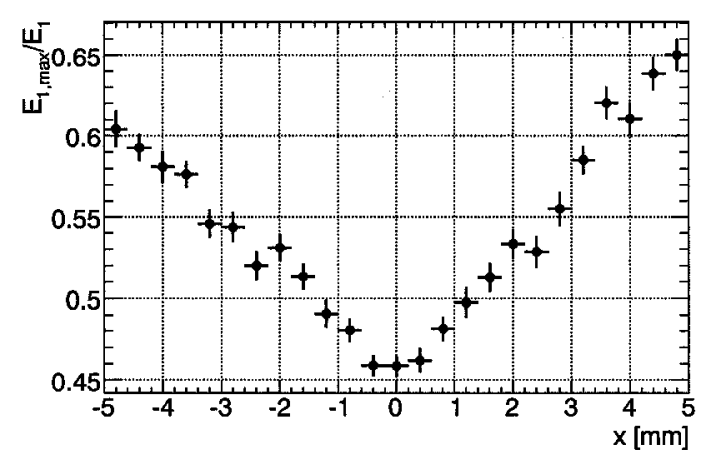

(a) Track scan in $\mathrm{x}$ of $E_{1, \max } / E_{1}$

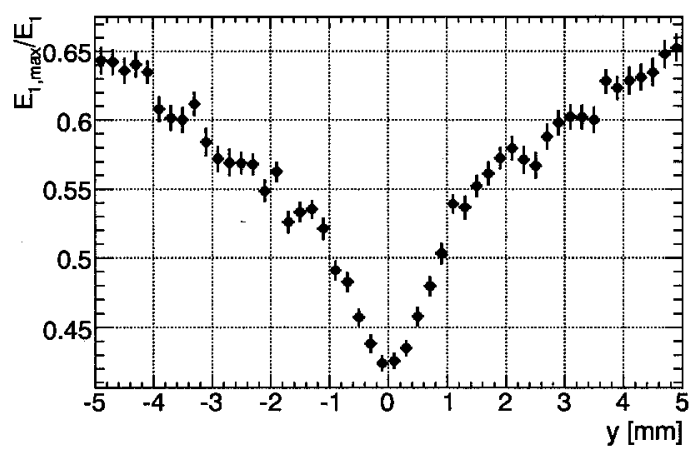

(b) Track scan in y of $E_{1, \max } / E_{1}$

Figure 7.3: Plots of $E_{1, \max } / E_{1}$ relative to $\mathrm{x}$ and $\mathrm{y}$ using the refined shift corrections. 
order to clearly see the minima from the $E_{1, \max } / E_{1} \mathrm{x}$ and $\mathrm{y}$ scans. This works well for electrons since their showers are contained within a few readout cells. In contrast, hadrons deposit their energy over a large number of readout cells which effectively smears the minima. Therefore, only electron data were used to study the track alignment stability. Results in Table 7.3 show the track shifts for energies between 40 and $200 \mathrm{GeV}$. It should

\begin{tabular}{|c|c|c|}
\hline Run Number & Energy [GeV] & Corrected Track Shift $(\mathrm{x}, \mathrm{y})[\mathrm{mm}]$ \\
\hline \hline 3574 to 3579 & 40 & $248.90,0.73$ \\
2543 to 2547 & 80 & $249.34,1.06$ \\
2595 to 2605 & 100 & $249.73,1.07$ \\
2385 to 2389 & 150 & $250.77,0.63$ \\
2324 to 2329 & 200 & $250.43,0.85$ \\
1784 to 1789 & 200 & $250.81,0.88$ \\
\hline \hline Average & N/A & $250.00,0.87$ \\
\hline
\end{tabular}

Table 7.3: Corrected shifts for various electron beam runs at the $4 \mathrm{~L}$ position.

be noted that there appears to be a slight, $<0.5 \%$, energy dependence when determining the track shifts. For the remainder of this analysis, the average track shifts will be used.

\subsection{Clustering and Noise}

For both electrons and hadrons, the energy sum from cells within a cylindrical cluster around the direction of the beam particle was used. The procedure of summing cell energies in adjacent cells is generally referred to as clustering. In the context of the 2003 test beam, clustering was used to reduce and correct for electronic noise, while retaining the full energy of the particle shower. Clustering reduces the noise contribution since the electronic noise for an energy sum is proportional to the number of cells collected. Therefore, a larger radius cluster will collect a larger amount of noise signals which degrades the calculation of the energy resolution at low energies. The center of a cylinder cluster was determined by extrapolating the reconstructed beam track to the face of each FCal module. For each module, cells whose centroid were within the cylinder radius were added to the cluster. The centroid of a cell was calculated using the positions of the electrodes 
within the readout cell.

Clustering is of particular importance for determining the energy resolution. In the 2003 test beam, an added factor was the fact that the electronic noise was not constant throughout the energy scan. The measured energy resolution is given by the sum in quadrature of the signal and the noise resolution, as shown in Equation 7.3.

$$
\sigma_{\text {total }} \approx \sqrt{\sigma_{\text {signal }}^{2}+\sigma_{\text {noise }}^{2}}
$$

Therefore, if the noise component varies, the measured resolution does not have the same interpretation for all energies. It should be stressed, that in order to produce a clear interpretation of the energy resolution, the noise contribution must be accounted for. In this analysis, the electronic noise was taken into account by using random events to determine the noise in each cluster for each event and subtracting this in quadrature from the measured resolution to determine the true signal resolution.

The first step in this process was to calculate the noise for a single cell using random events. For these events, no event phase was available to select a set of OFCs, therefore random phases were used. By applying these OFCs to the random events and applying the sample calibrations, the reconstructed noise could be determined. For pions, the hadronic calibration must also be included in order to calculate the reconstructed noise.

For every run within this analysis, the random events were used to calculate the noise for each readout cell. Afterwards, the reconstructed noise was mapped to the clustered cell from the physics event. This ensured that the reconstructed noise used only the cells that were included in the clusters on an event-by-event basis. It was important to use the full reconstruction chain as the reconstructed noise was $\sim 30 \%$ smaller than the raw noise due to the optimal filtering technique. 
The noise resolution, $\sigma_{\text {noise }}$, was determined using the standard deviation from a single Gaussian fit to the noise distribution. The last step in the noise correction procedure was to subtract the noise resolution in quadrature from the fitted energy resolution for each energy.

\subsection{Electron Performance}

As indicated above, beam cleaning and beam envelope cuts were applied in order to remove beam particles that had scattered or had hadron contamination. The tail-catcher was used to remove muon and hadron contamination and the muon scintillator was used to further suppress muons. In addition, a CEDAR cut was applied to the $60 \mathrm{GeV}$ beam energy events to further suppress hadron contamination in the beam. This section presents the detector performance results for electron beam data using measurements from the electromagnetic module, FCal 1.

The electron performance of the FCal 1 calorimeter was studied by summing the energy from channels within a defined cylinder cluster size. An $8 \mathrm{~cm}$ cluster was the smallest cluster used in this analysis and corresponds to $\sim 4.7$ Molière radius ensuring nearly $100 \%$ shower containment. Three cluster sizes, 8,12 and $16 \mathrm{~cm}$, were investigated in order to estimate the systematic error associated with the clustering and-noise technique. To determine the energy resolution for FCal 1, data from the electron runs for energies between 10 and $200 \mathrm{GeV}$ were used. The response for each electron beam energy for an $8 \mathrm{~cm}$ cluster size is shown in Figures 7.4(a) to 7.4(h). The energy units in these figures are quoted in ADC which are the digitized measurements of the analog signals. These digitized measurements are proportional to the energy deposited. The mean energy and resolution for each beam energy was determined by fitting the energy distribution with a double Gaussian. This was motivated by the non-symmetric shape of the energy dis- 
tribution, which resulted from the unique geometry of the FCal modules. In addition, although there was an attempt to remove hadron contamination, there was still a noticeable amount that survived all cuts as can be seen in Figure 7.4(g) which shows the hadronic contamination peak at 1000 ADC. Therefore, in addition to the double Gaussian used to fit the signal distribution, the hadron background was modeled using hadron data with the same beam energy. The final fitting function consisted of seven parameters, six for the double Gaussian which parameterized the signal and one amplitude normalization factor needed to fit the hadron background data ${ }^{3}$.

\subsubsection{Electron Energy Linearity}

One of the primary goals of the 2003 test beam is to determine the characteristics of the FCal modules, such as the electromagnetic scale, linearity, and energy resolution. The readout electronics used in the test beam were not the same as the ones that are being used in ATLAS as they were not available in time. As a result, a SPICE ${ }^{4}$ model of the readout electronics will provide the initial calibration value of the energy response from ADC to $\mathrm{GeV}$. However, in order to gain confidence in the SPICE model, the prediction of the energy response for the 2003 test beam electronics for FCal 1 was compared to the electron results presented in this section. The reconstructed energy was calculated using the first moment of the fitted double Gaussian, as shown in Equation 7.4.

$$
<E>=\int_{\min }^{\max } E \mathcal{G}_{1}(E) \mathcal{G}_{2}(E) d E
$$

Here, $\mathcal{G}_{i}(E)$ is the $i^{\text {th }}$ Gaussian distribution and $E$ is the energy. The $\min$ and $\max$ were set to the minimum and maximum values of the energy distribution histogram. It should be noted that several other fitting procedures were investigated, such as a single Gaussian function, producing similar results. The results of the mean reconstructed energy from

\footnotetext{
${ }^{3}$ No pion data was taken at $20 \mathrm{GeV}$, therefore no pion modeling was performed for the $20 \mathrm{GeV}$ electron point.

${ }^{4}$ SPICE is a software tool which simulates and analyses electronic circuits ranging from simple single resistor circuits to sophisticated circuits comprising tens-of-thousands of devices.
} 


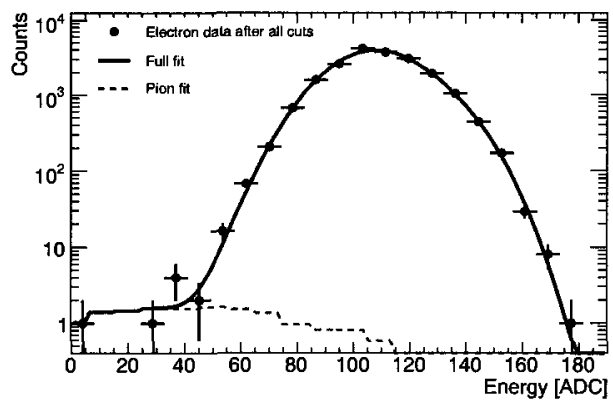

(a) $10 \mathrm{GeV}$ Electron

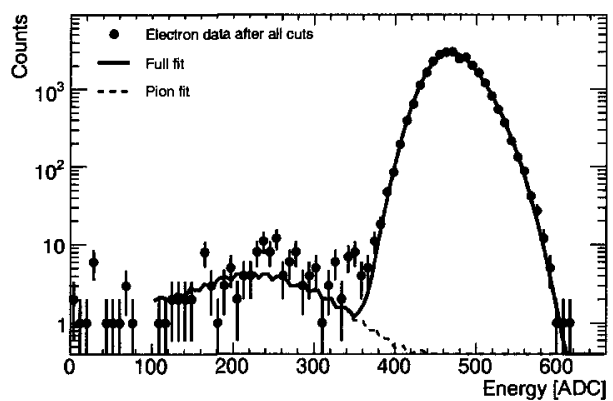

(c) $40 \mathrm{GeV}$ Electron

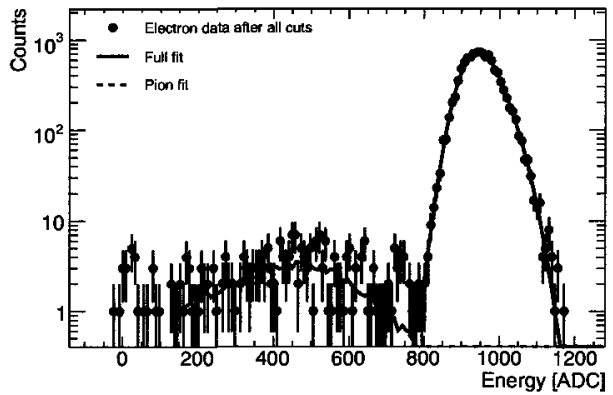

(e) $80 \mathrm{GeV}$ Electron

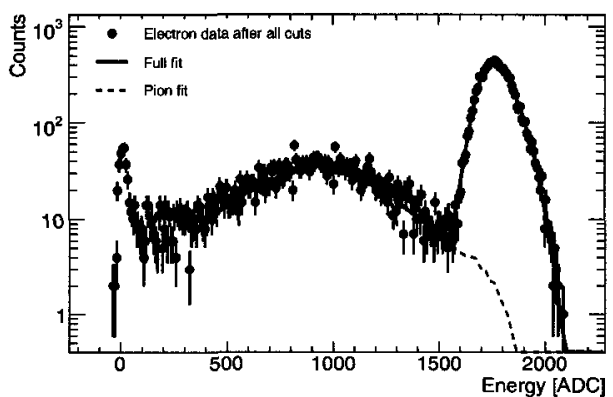

(g) $150 \mathrm{GeV}$ Electron

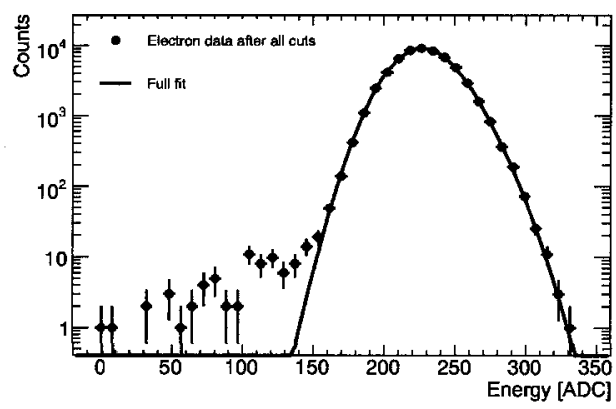

(b) $20 \mathrm{GeV}$ Electron

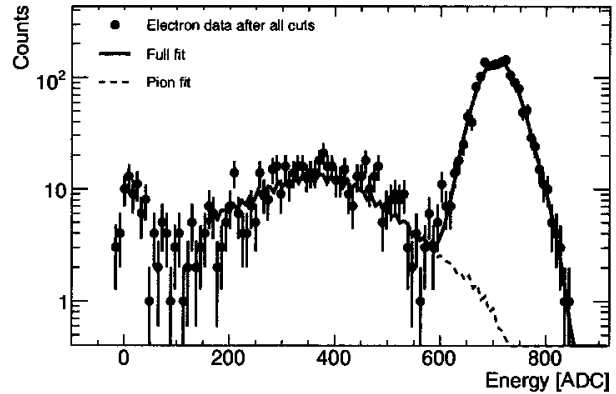

(d) $60 \mathrm{GeV}$ Electron

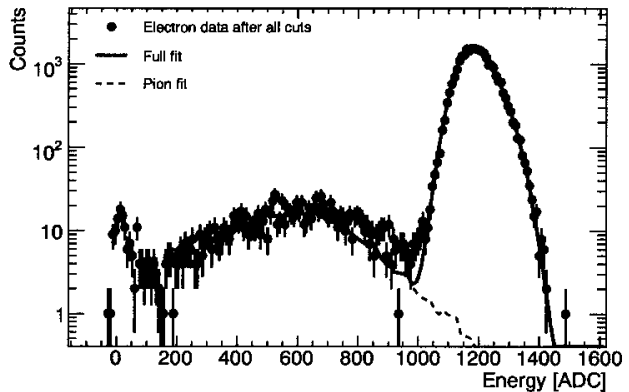

(f) $100 \mathrm{GeV}$ Electron

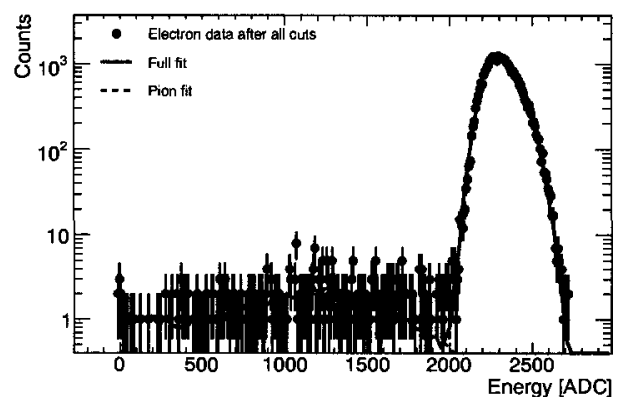

(h) $200 \mathrm{GeV}$ Electron

Figure 7.4: Energy distribution for electrons in the 4L position using an $8 \mathrm{~cm}$ cylinder cluster. The bullets are electron data, the solid line is the 7 parameter fit, and the dashed line represents the pion fit. Note that the $20 \mathrm{GeV}$ pion data was not available for the background fit. 
the energy distribution using the fitting procedure defined in Section 7.4 are presented in Table 7.4. The results in Table 7.4 show that the individual electron energy response sys-

\begin{tabular}{|c|c|c|c|c|c|c|}
\hline \multirow{2}{*}{$\begin{array}{c}\text { Beam Energy } \\
{[\mathrm{GeV}]}\end{array}$} & \multicolumn{3}{|c|}{ Mean $[\mathrm{ADC}]$} & \multirow{2}{*}{$\begin{array}{c}\text { Mean Average } \\
\text { [ADC] }\end{array}$} & \multirow{2}{*}{$\begin{array}{l}\text { RMS } \\
{[\mathrm{ADC}]}\end{array}$} & \multirow{2}{*}{$\begin{array}{c}\text { RMS } \\
{[\%]}\end{array}$} \\
\hline & $8 \mathrm{~cm}$ & $12 \mathrm{~cm}$ & $16 \mathrm{~cm}$ & & & \\
\hline 10 & 109.25 & 109.91 & 110.30 & 109.82 & 0.528 & 0.48 \\
\hline 20 & 228.40 & 230.05 & 230.10 & 229.52 & 0.968 & 0.42 \\
\hline 40 & 472.64 & 476.34 & 477.21 & 475.40 & 2.429 & 0.51 \\
\hline 60 & 709.58 & 714.96 & 716.27 & 713.60 & 3.547 & 0.50 \\
\hline 80 & 951.43 & $\begin{array}{l}958.92 \\
\end{array}$ & $\begin{array}{l}960.09 \\
\end{array}$ & 956.81 & 4.696 & 0.49 \\
\hline 100 & $1,193.32$ & $1,202.94$ & $1,204.56$ & $1,200.27$ & 6.076 & 0.51 \\
\hline 150 & $1,776.03$ & $1,789.41$ & $1,791.84$ & $1,785.76$ & 8.514 & 0.48 \\
\hline 200 & $2,319.85$ & $2,337.09$ & $2,340.11$ & $2,332.35$ & 10.930 & 0.47 \\
\hline
\end{tabular}

Table 7.4: Cluster size results for the electron reconstructed energy. Columns 2-4 are the reconstructed energy in ADC for three diffrent cluster size. Column 5 is the average of the means and columns 6-7 are the rms of the means.

tematically grows as the cluster size increases, this increase is less than $\sim 1 \%$. In addition, the results show that the RMS of the mean reconstructed energy for all cluster sizes for all electron energies is small, approximately $0.5 \%$. The overall electron energy response of the FCal 1 module for all beam energies was determined using a linear fit and is presented in Figure 7.5(a) and Table 7.5. The results in Table 7.5 show that the ADC/GeV conversion factor systematically grows as the cluster size increases, consistent with the results in Table 7.4. In addition, the residual of the linear fit is presented in Figure 7.5(b) and is less than $0.5 \%$ for beam energies of $20 \mathrm{GeV}$ or greater.

At low energies, $E_{\text {Beam }} \leq 20 \mathrm{GeV}$, data was taken using electrons and positrons and a small difference between the mean energy response for electrons and positrons was noticed. The difference was found to be $0.9 \%$ at $10 \mathrm{GeV}$ and $0.6 \%$ at $20 \mathrm{GeV}$ which is similar to the fluctuations observed at these energies shown in Figure 7.5(b).

Using the average results from the cylinder clustering provides an electron response of $12.14 \pm 0.06$ (sys) \pm 0.002 (stat) ADC/GeV. Here, the systematic error was estimated using the RMS of the slope from the three different clustering sizes in Table 7.5. The electron 


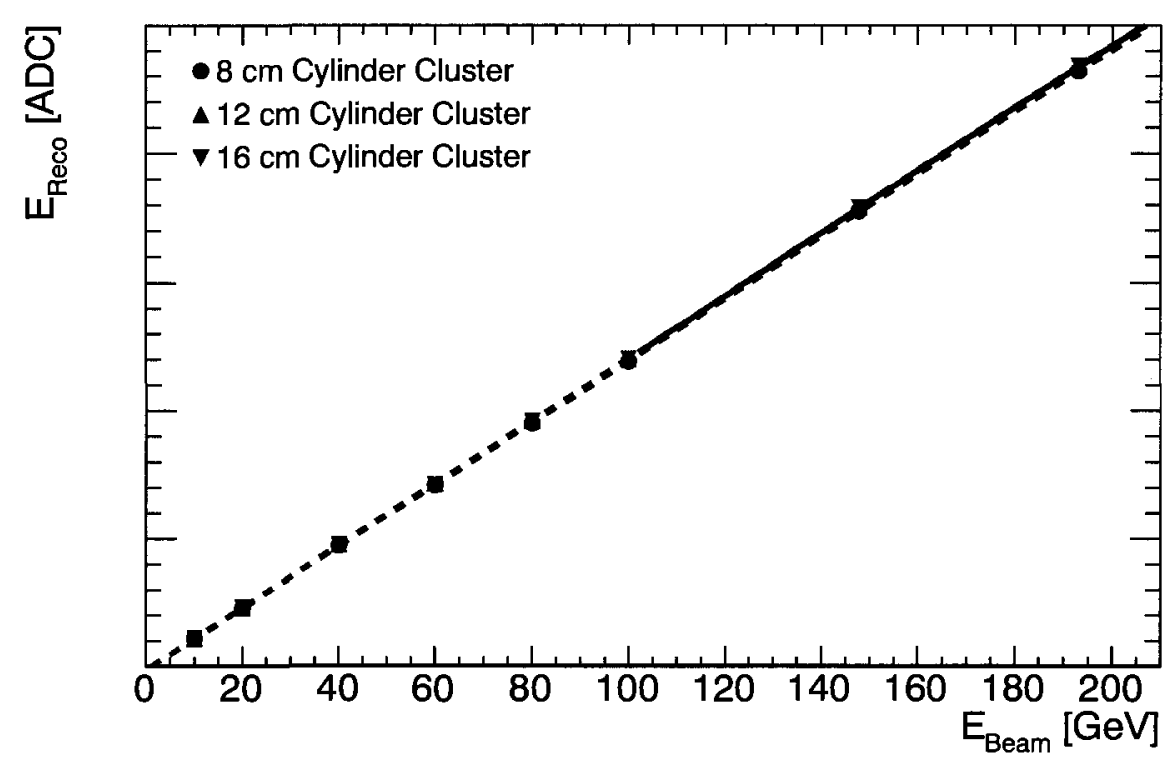

(a) Electron energy linearity.

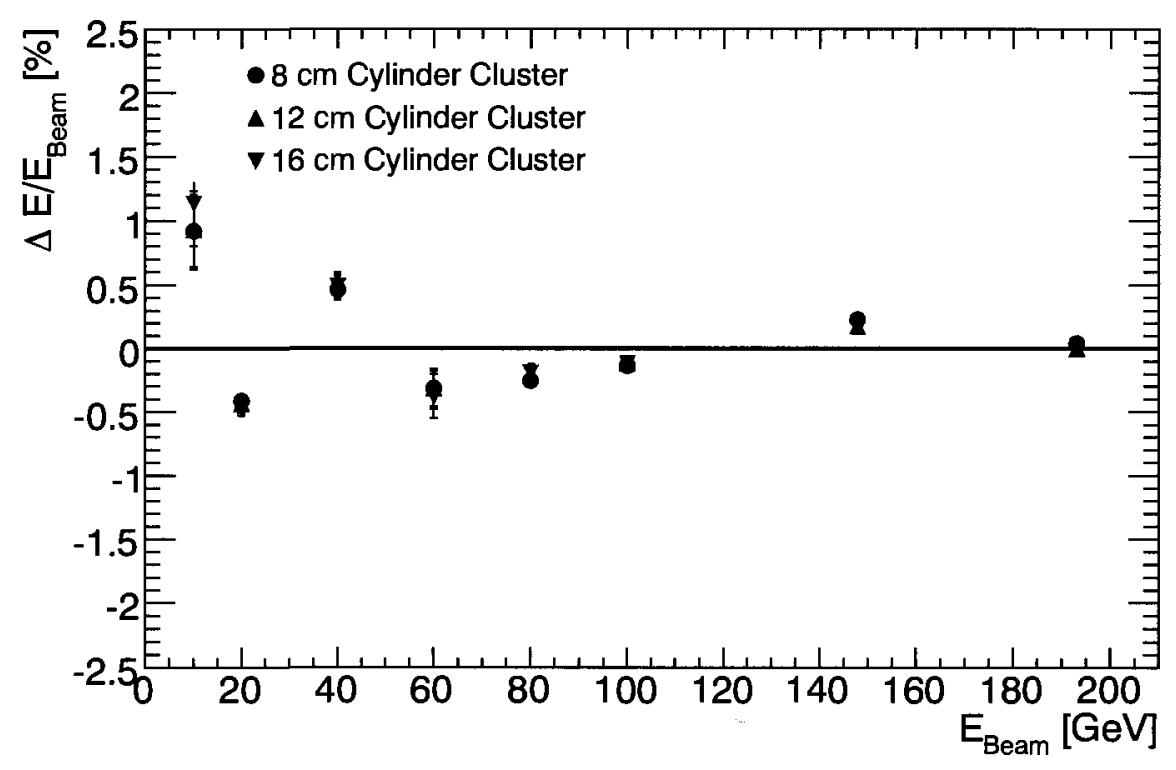

(b) Residual of the energy linearity fit.

Figure 7.5: Electron energy linearity plot 7.5(a) with residual of the energy linearity fit 7.5(b) using various cylinder cluster sizes. 
response predicted by the SPICE model is $12 \mathrm{ADC} / \mathrm{GeV}$ which is in good agreement with the results of this thesis. The intercept in Figure 7.5(a) from the linear fit is non zero and is attributed to energy losses upstream. The intercept is equivalent to approximately $1 \mathrm{GeV}$.

\begin{tabular}{|c|c|c|}
\hline Cylinder size [cm] & Slope [ADC/GeV] & Intercept [ADC] \\
\hline \hline 8 & $12.066 \pm 0.002$ & $-12.185 \pm 0.067$ \\
\hline 12 & $12.161 \pm 0.002$ & $-12.451 \pm 0.081$ \\
\hline 16 & $12.178 \pm 0.002$ & $-12.448 \pm 0.096$ \\
\hline
\end{tabular}

Table 7.5: Electron energy linearity for three different cylinder cluster size.

\subsubsection{Electron Energy Resolution}

The energy resolution of the calorimeter was studied by summing the energy from channels within a defined cylinder cluster size. The reconstructed resolution for each electron energy was determined using moment calculations of the fitted double Gaussian, as shown in Equation 7.5.

$$
\sigma_{E}=\sqrt{\int_{\min }^{\max } E^{2} \mathcal{G}_{1}(E) \mathcal{G}_{2}(E) d E-\left[\int_{\min }^{\max } E \mathcal{G}_{1}(E) \mathcal{G}_{2}(E) d E\right]^{2}}
$$

Here, $\mathcal{G}_{i}(E)$ is the $i^{t h}$ Gaussian distribution and $E$ is the energy. The min and max were set to the minimum and maximum values of the energy distribution histogram. As with the reconstructed mean energy several fitting procedures were investigated producing similar results.

The calorimeter energy resolution as a function of beam energy was parameterized using Equation 7.6.

$$
\frac{\sigma_{E}}{E}=\frac{a}{\sqrt{E_{\text {beam }}}} \oplus b
$$

Here, $E$ is the mean energy calculated using Equation $7.4, \sigma_{E}$ is the reconstructed resolution calculated using Equation 7.5, and $a$ and $b$ are the stochastic and constant terms. 
In order to understand the systematic error associated with the noise subtraction technique, several cluster sizes were investigated. The results are presented in Table 7.6 and Figure 7.6(a). In addition, the noise values subtracted in quadrature from the measured

\begin{tabular}{|c|c|c|c|c|c|c|}
\hline \multirow{2}{*}{$\begin{array}{c}\text { Beam Energy } \\
{[\mathrm{GeV}]} \\
\end{array}$} & \multicolumn{3}{|c|}{ Resolution $[\% / \mathrm{GeV}]$} & \multirow{2}{*}{$\begin{array}{c}\text { Resolution Average } \\
{[\% / \mathrm{GeV}]} \\
\end{array}$} & \multirow{2}{*}{$\begin{array}{c}\text { RMS } \\
{[\% / \mathrm{GeV}]}\end{array}$} & \multirow{2}{*}{$\begin{array}{c}\text { RMS } \\
{[\%]} \\
\end{array}$} \\
\hline & $8 \mathrm{~cm}$ & $12 \mathrm{~cm}$ & $16 \mathrm{~cm}$ & & & \\
\hline 10 & 9.62 & 9.58 & 9.65 & $\overline{9.62}$ & 0.039 & 0.41 \\
\hline 20 & 7.21 & 7.26 & 7.41 & 7.29 & 0.102 & 1.39 \\
\hline 40 & 5.72 & 5.57 & 5.59 & 5.63 & 0.082 & 1.46 \\
\hline 60 & 4.91 & 4.89 & 5.01 & 4.93 & 0.064 & 1.29 \\
\hline 80 & 4.79 & 4.76 & 4.76 & 4.77 & 0.018 & 0.37 \\
\hline 100 & 4.62 & 4.59 & 4.65 & 4.62 & 0.032 & 0.69 \\
\hline 150 & 4.21 & 4.20 & 4.11 & 4.17 & 0.053 & 1.27 \\
\hline 200 & 4.07 & 4.03 & 4.03 & 4.04 & 0.023 & 0.56 \\
\hline
\end{tabular}

Table 7.6: Results of the effects of cluster size on electron energy resolution. Column 2-4 are the corrected energy resolution for three different cluster sizes.

resolutions are presented in Figure 7.6(b). The RMS of the energy resolution over all cluster sizes is less than $\sim 1.5 \%$ for all electron energies and provides an estimation of the systematic error associated with the cluster and noise subtraction techniques. Using the average results from the cylinder clustering in Table 7.7 provides an electron energy resolution with a stochastic term $a=27.96 \pm 0.6$ (sys) $\pm 0.18($ stat $) \% \cdot \sqrt{\mathrm{GeV}}$ and a constant term $b=3.48 \pm 0.13$ (sys) \pm 0.02 (stat)\%. Here, the systematic error for the stochastic (constant) term was estimated using the RMS of the stochastic (constant) terms from the three different clustering sizes in Table 7.7. These results are consistent with the published FCal test beam results $[23,26]$.

\begin{tabular}{|c|c|c|}
\hline Cylinder size $[\mathrm{cm}]$ & Sampling (a) & Constant (b) \\
\hline \hline 8 & $27.28 \pm 0.11$ & $3.59 \pm 0.01$ \\
\hline 12 & $27.73 \pm 0.15$ & $3.52 \pm 0.01$ \\
\hline 16 & $28.86 \pm 0.18$ & $3.34 \pm 0.02$ \\
\hline
\end{tabular}

Table 7.7: Electron energy resolution for three different cluster size. 


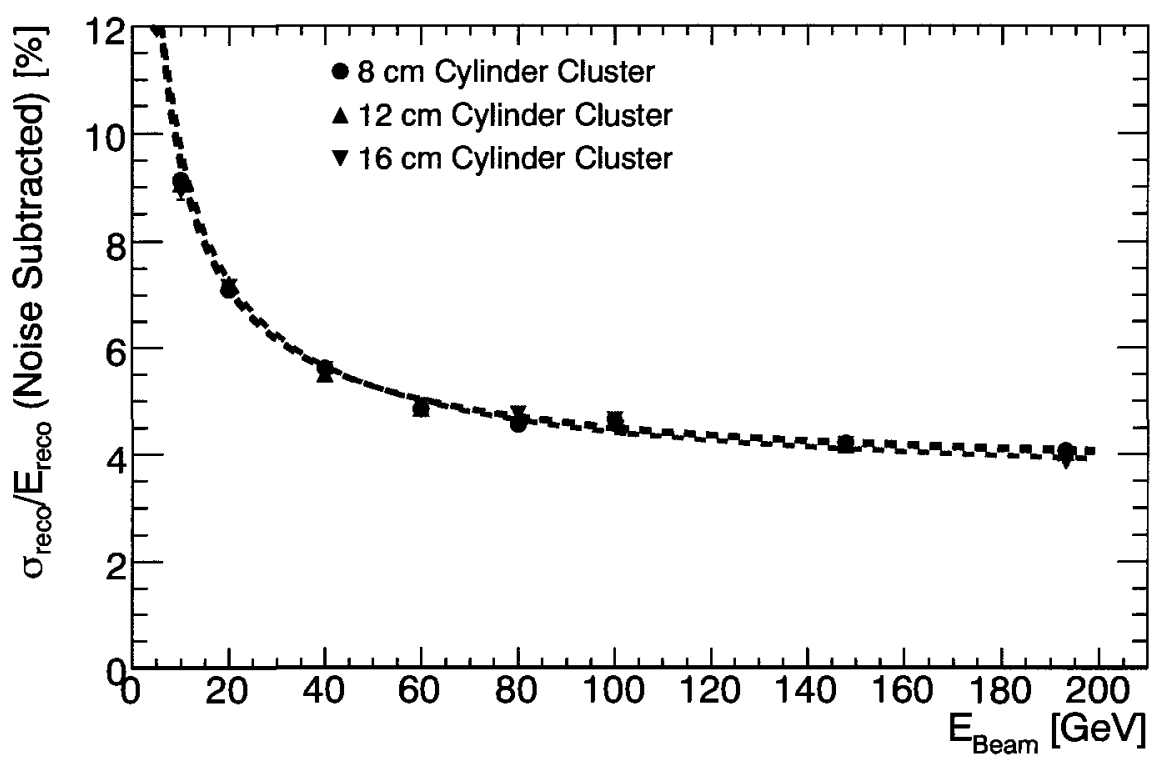

(a) Electron energy resolution.

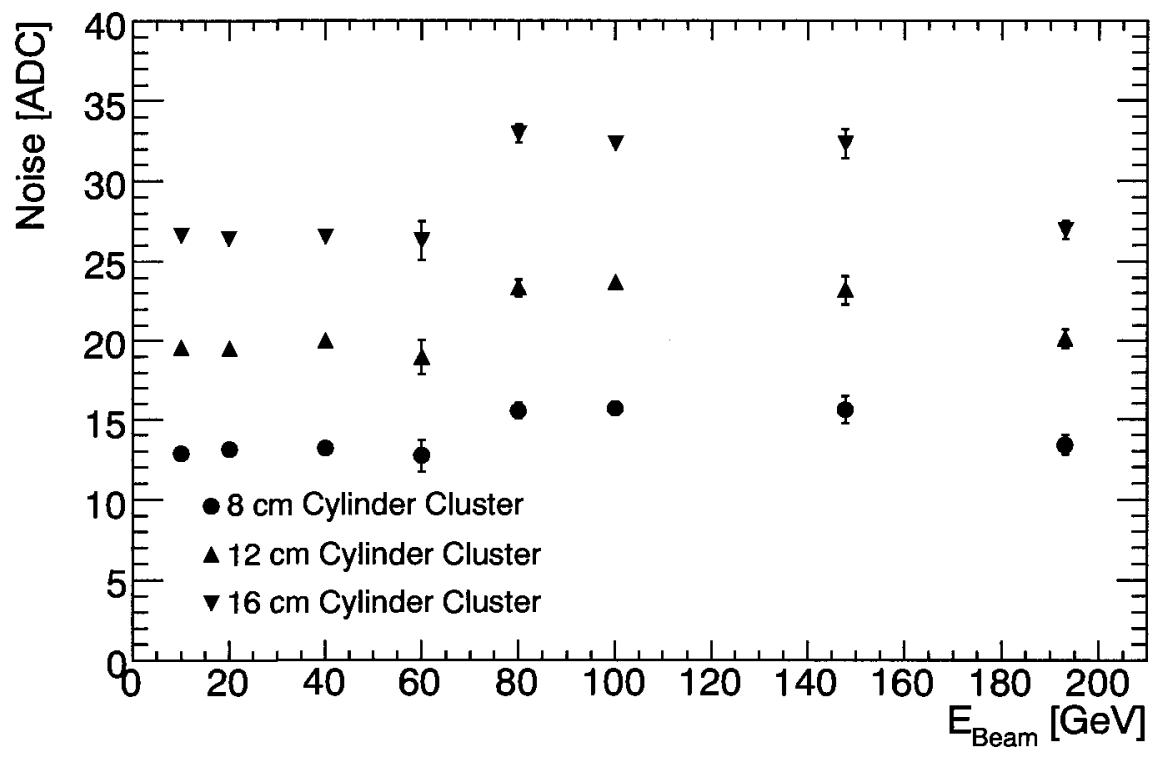

(b) Electron reconstructed noise resolution. The rise in noise between $80-150 \mathrm{GeV}$ is associated to the noise problem summarized in Section 5.2.

Figure 7.6: Electron energy resolution results are shown in Figure 7.6(a) with noise subtracted in quadrature using noise values shown in Figure 7.6(b) using various cylinder cluster sizes. 


\subsection{Hadron Performance}

The hadronic response of the FCal module was determined using a pion beam for energies between 10 and $200 \mathrm{GeV}$. This provides an estimation for the performance of the FCal detectors for jets. As with electrons, the mean energy response and resolution were determined by fitting the data with a double Gaussian. In addition, the mean energy response and the energy resolution at each beam energy was calculated using Equations 7.4 and 7.5 , respectively. A cluster size of $16 \mathrm{~cm}$ was used for this analysis. An example of the pion energy distributions using transverse weights are shown in Figures 7.7(a) to 7.7(h).

\subsubsection{Compensation Techniques}

A calorimeter is said to be compensating if electrons and hadrons have the same energy response. Reasonable compensation can be achieved by carefully choosing the material used to construct the detector. If the calorimeter is not compensating by design, as is the case with the FCal modules, offline software corrections can be applied to achieve a more uniform energy response. In this section, two software techniques used to provide better uniformity in the FCal energy response are discussed. These techniques are the baseline technique (Section 7.5.2) and the transverse weighting technique (Section 7.5.3).

To determine the calibration weights used to reconstruct the pion energy, both techniques use the following minimizing ${ }^{5}$ Equation 7.7. However, the definition of the reconstructed energy is different for each technique and will be discussed in Sections 7.5.2 and 7.5.3.

$$
\chi^{2}=\sum_{i=1}^{n}\left(E_{\text {beam }}-E_{i}\right)^{2}
$$

Here, $E_{\text {beam }}$ is the beam energy, $E_{i}$ is the reconstructed energy for the $i^{\text {th }}$ event, and $n$ is the total number of events after cuts. In the above mentioned minimization, only the energy resolution is optimized. However, it is desired to also optimize the energy scale.

\footnotetext{
${ }^{5}$ The error is assumed to be equal for all events used in the minimization and is omitted.
} 


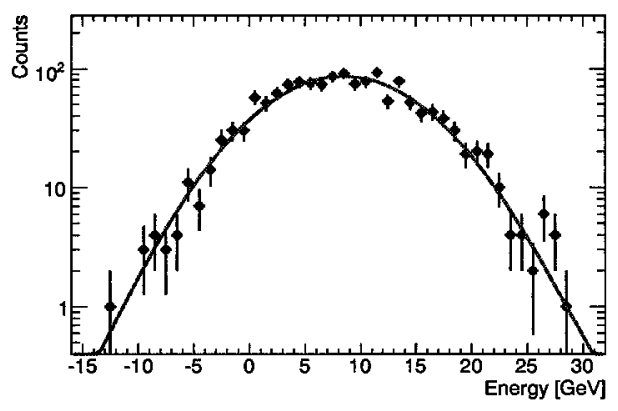

(a) $10 \mathrm{GeV}$ Pion

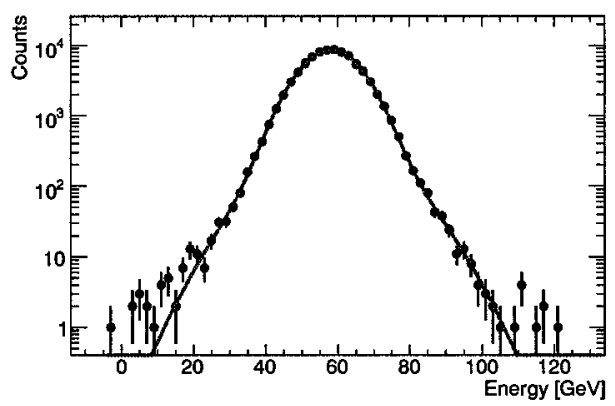

(c) $60 \mathrm{GeV}$ Pion

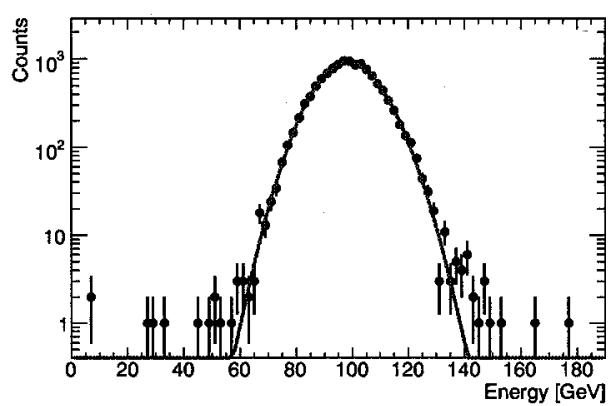

(e) $100 \mathrm{GeV}$ Pion

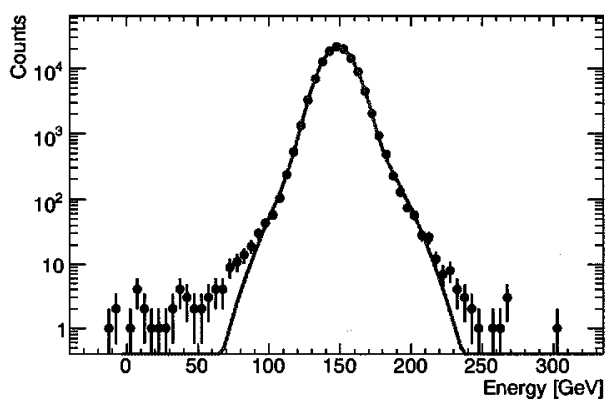

(g) $150 \mathrm{GeV}$ Pion

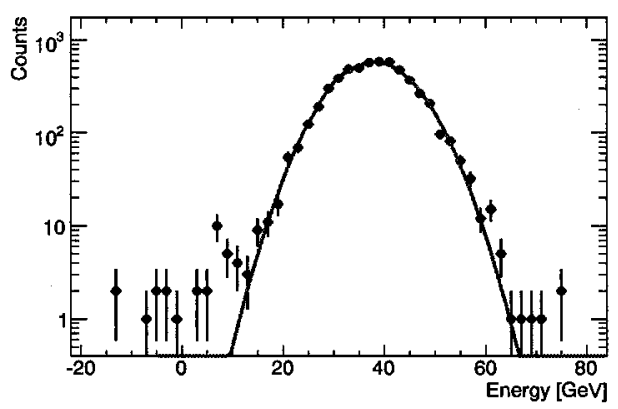

(b) $40 \mathrm{GeV}$ Pion

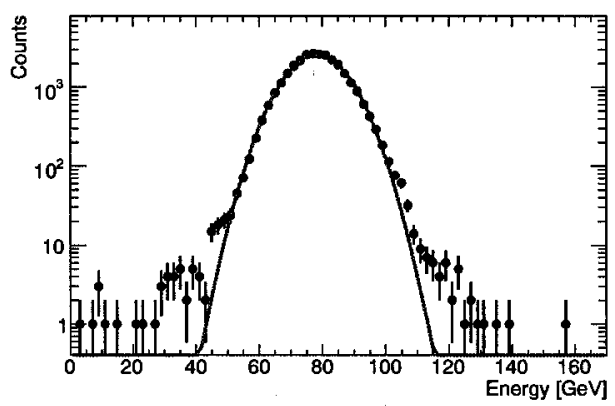

(d) $80 \mathrm{GeV}$ Pion

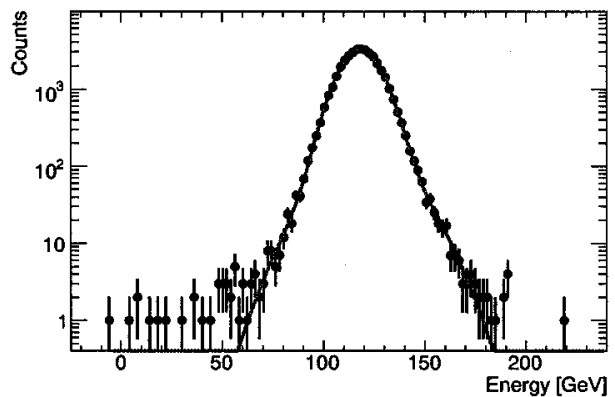

(f) $120 \mathrm{GeV}$ Pion

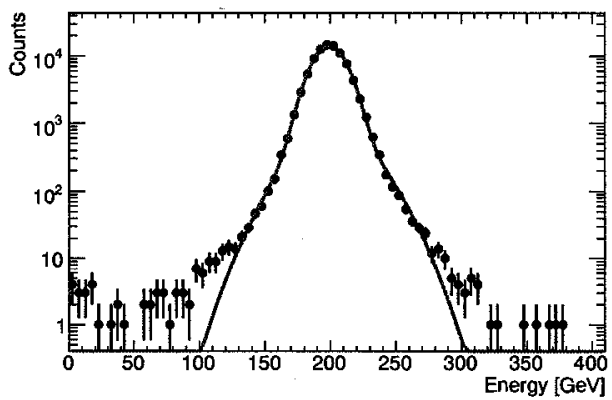

(h) $200 \mathrm{GeV}$ Pion

Figure 7.7: Energy distribution for pions in the $4 \mathrm{~L}$ position using a $16 \mathrm{~cm}$ cylinder cluster and transverse weights. The points are pion data, the solid line is the 6 parameter fit. 
Therefore it is common to constrain the minimization procedure by requiring the mean reconstructed energy to be equal to the beam energy.

\subsubsection{Baseline Approach}

For the baseline approach, the energy of each FCal module is multiplied by a single calibration term resulting in the reconstructed energy defined in Equation 7.8.

$$
E=\alpha_{1} \cdot E_{F C a l 1}+\alpha_{2} \cdot E_{F C a l 2}+\alpha_{3} \cdot E_{F C a l 3}
$$

Here, $E_{F C a l 1,2,3}$ is the clustered energy for each FCal module in GeV and the $\alpha$ terms are free parameters determined during the minimization and are generally referred to as flat weights. The ADC to $\mathrm{GeV}$ conversion factor for FCal 2 and FCal 3 was determined using the same SPICE model as used for FCal 1. The prediction for the FCal 2 was $6.1 \mathrm{ADC} / \mathrm{GeV}$ and 5.4 ADC/GeV for FCal 3. For FCal 2, this prediction is consistent to within $\sim 5 \%$ of previous test beam results [23].

In order to determine the systematics of this weighting technique, weights were derived for beam energies of $100,120,150$, and $200 \mathrm{GeV}$. To optimize the overall calibration performance, a constraint was used, requiring the mean of the reconstructed energy to be equal to the energy of the beam, as shown in Equation 7.9.

$$
\sum \alpha_{i}<E_{i}>=E_{B e a m}
$$

Results from the constrained and unconstrained minimizations using the same energies as noted above are presented in Table 7.8. From this table it can seen that the flat weights are energy dependent. The $\alpha_{1}$ term decreases with energy while the $\alpha_{3}$ term increases with energy. These systematic variations are believed to be due to the difference in the fractional amount of energy deposited in the FCal modules. 


\begin{tabular}{|c|c|c|c|c|c|c|c|}
\hline \multirow{2}{*}{$\begin{array}{c}\text { Calibration } \\
\text { Weights }\end{array}$} & \multirow{2}{*}{ Fit Type } & \multicolumn{4}{|c|}{ Energy [GeV] } & \multirow{2}{*}{ Mean } & RMS \\
\cline { 3 - 7 } & & 100 & 120 & 150 & 200 & & {$[\%]$} \\
\hline \hline \multirow{2}{*}{$\alpha_{1}$} & Unconstrained & 1.268 & 1.258 & 1.241 & 1.221 & 1.247 & 1.653 \\
\cline { 2 - 7 } & Constrained & 1.294 & 1.277 & 1.258 & 1.234 & 1.266 & 2.036 \\
\hline \multirow{2}{*}{$\alpha_{2}$} & Unconstrained & 1.216 & 1.218 & 1.216 & 1.202 & 1.213 & 0.610 \\
\cline { 2 - 7 } & Constrained & 1.240 & 1.237 & 1.232 & 1.216 & 1.231 & 0.868 \\
\hline \multirow{2}{*}{$\alpha_{3}$} & Unconstrained & 1.369 & 1.425 & 1.422 & 1.440 & 1.414 & 2.194 \\
\cline { 2 - 7 } & Constrained & 1.400 & 1.447 & 1.441 & 1.456 & 1.436 & 1.726 \\
\hline
\end{tabular}

Table 7.8: Results from flat weight fits with and without beam energy constraints.

\begin{tabular}{|c|c|c|c|c|c|c|c|c|}
\hline \multirow{2}{*}{$\begin{array}{c}\text { Resolution } \\
\text { Terms } \\
\end{array}$} & \multirow{2}{*}{ Fit Type } & \multicolumn{5}{|c|}{ Energy used to Derive Flat Weights [GeV] } & \multirow{2}{*}{ Mean } & \multirow{2}{*}{ RMS } \\
\hline & & 100 & 120 & 150 & 200 & $100-200$ & & \\
\hline \multirow{2}{*}{$\begin{array}{l}\text { Stochastic } \\
{[\% \cdot \sqrt{\mathrm{GeV}}]}\end{array}$} & $\overline{\mathrm{Un}}$ & 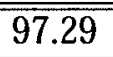 & 97.53 & $\overline{103.49}$ & 100.49 & 98.61 & & 2.57 \\
\hline & Con: & 98.43 & 98.17 & 102.30 & 98.25 & 98.41 & 99.11 & 1.78 \\
\hline \multirow{2}{*}{$\begin{array}{c}\text { Constant } \\
{[\%]}\end{array}$} & Unc & 7.18 & 6.95 & 6.22 & 6.69 & 6.97 & 6.80 & 0.37 \\
\hline & Constrained & 7.08 & 7.12 & 6.91 & 7.13 & 7.12 & 7.07 & 0.09 \\
\hline
\end{tabular}

Table 7.9: Values determined for the stochastic and constant terms using different sets of flat weights derived with and without beam energy constraints.

From the results in the last column in Table 7.9 , the constrained flat weights provide a smaller variation in stochastic and constant terms throughout the full range of weights derived at different energies. In addition, the energy response improves as shown in Figure 7.8. The uncertainty in this analysis is dominated by the systematic error, of which the largest source comes from the calibration weights. Using beam energy constrained flat weights derived from the $200 \mathrm{GeV}$ data a hadron energy resolution with a stochastic term $a=98.25 \pm 1.78($ sys $) \pm 0.50($ stat $) \% \cdot \sqrt{\mathrm{GeV}}$ and a constant term $b=7.13 \pm 0.09($ sys $) \pm$ 0.004 (stat) $\%$ is obtained. Here, the systematic error for the stochastic (constant) term was estimated using the RMS of the stochastic (constant) terms shown in Table 7.9 using constrained flat weights derived at the four different energies.

\subsubsection{Transverse Weights}

In this section, a more advanced hadronic calibration technique is presented. The transverse weighting technique was developed to take advantage of the FCal's unique fine segmentation in $\mathrm{x}$ and $\mathrm{y}$. For the transverse weighting technique, the reconstructed en- 


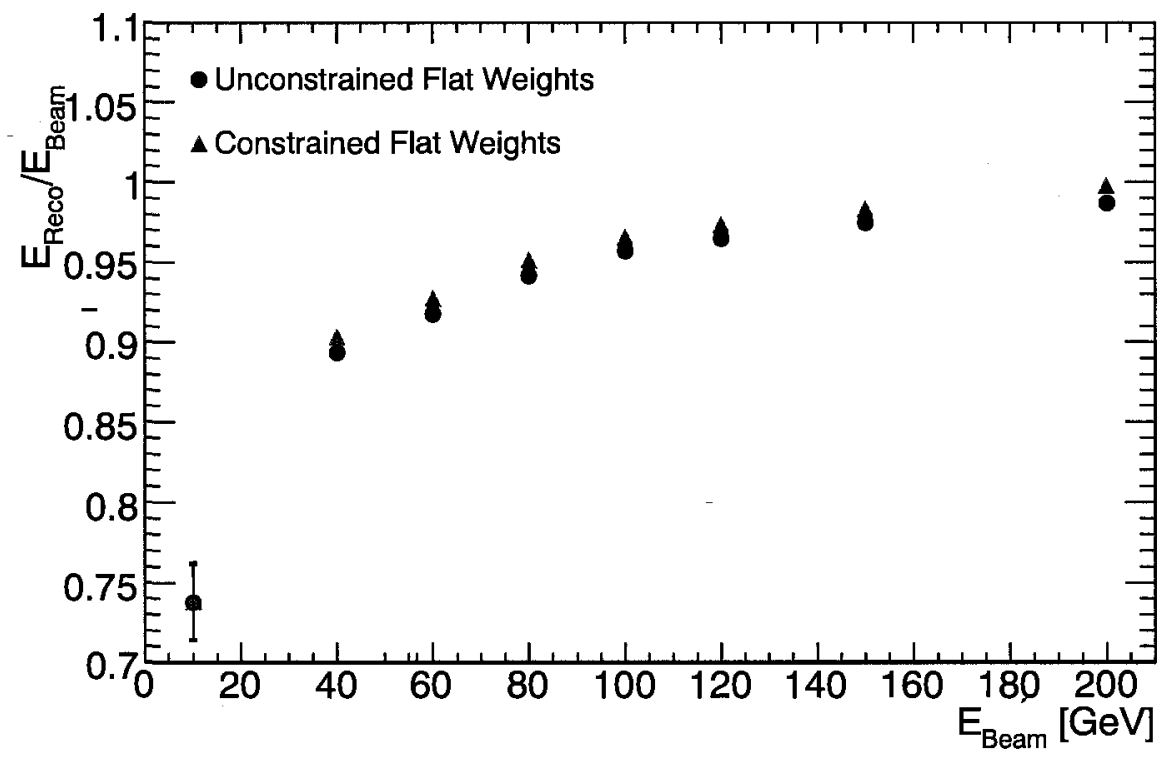

Figure 7.8: Comparison of the energy response using flat weights derived from a 200 $\mathrm{GeV}$ pion beam data with and without beam constraints as a function of beam energy. The ratio between the reconstructed energy and the beam energy, $E_{\text {Reco }} / E_{\text {Beam }}$, was used to quantify the beam response. 
ergy is defined using Equation 7.10.

$$
E=\sum_{j} \sum_{i} E_{i j} \times W_{j}\left(R_{i j}\right)
$$

Here, $E_{i j}$ is the energy of the $i^{\text {th }}$ readout cell in the $j^{\text {th }}$ FCal module for a single event and $W_{j}\left(R_{i j}\right)$ is the transverse weight associated with the module of the readout cell. The $R_{i j}$ variable is the distance between the centroid of the $i^{t h}$ cell and the shower center of the $j^{\text {th }}$ module, as defined in Equation 7.11.

$$
R_{i j}=\sqrt{\left(x_{i}-x_{j}\right)^{2}+\left(y_{i}-y_{j}\right)^{2}}
$$

The transverse weights applied to the data are determined by linear interpolation between a set of transverse weights $W_{j}\left(R_{d}\right)$ at discrete distances $R_{d}$, where $d \in(1, \cdots, N)$ using Equation 7.12.

$$
W_{j}\left(R_{i j}\right)= \begin{cases}W\left(R_{1}\right) & \text { if } R_{i j}<R_{1} \\ W\left(R_{N}\right) & \text { if } R_{i j}>R_{N} \\ W\left(R_{d}\right) \times \frac{R_{d+1}-R_{i j}}{R_{d+1}-R_{d}}+W\left(R_{d+1}\right) \times \frac{R_{i j}-R_{d}}{R_{d+1}-R_{d}} & \text { otherwise }\end{cases}
$$

In this study, 16 weights were used for each module spaced at $1 \mathrm{~cm}$ intervals.

In order to estimate the systematic error, weights were generated as described above using 120,150, and $200 \mathrm{GeV}$ energy hadrons. The transverse weight distributions are shown in Figure 7.9. These distributions can be understood as follows: the energy closest to the shower center is dominated by the electromagnetic shower core resulting in a weight near 1. As the shower proliferates radially outwards, the fractional hadronic contribution increases. In order to achieve compensation, the weights also increase. Finally, far from the shower center, there is a reduction in the deposited energy from the particle shower and the noise contribution starts to dominate. This results in decreased weight values. The 
variation in the amplitude and shape of the transverse weight distribution between each FCal module can be understood by the location of each module relative to the shower development and the variation in the detector absorber material. It is clear from the energy resolution results using transverse weights presented in Table 7.10, that this weighting technique provides superior performance over the flat weight results presented in Section 7.5.2. Using unconstrained transverse weights derived from the $200 \mathrm{GeV}$ data provides a

\begin{tabular}{|c|c|c|c|c|c|}
\hline \multirow{2}{*}{$\begin{array}{c}\text { Resolution } \\
\text { Terms }\end{array}$} & \multicolumn{3}{|c|}{ Energy used to Derive Weights $[\mathrm{GeV}]$} & \multirow{2}{*}{ Mean } & \multirow{2}{*}{ RMS } \\
\cline { 2 - 5 } & 120 & 200 & Average $(120,150,200)$ & & \\
\hline \hline Stochastic $[\% \cdot \sqrt{\mathrm{GeV}}]$ & 70.89 & 72.63 & 72.86 & 72.13 & 1.08 \\
\hline Constant $[\%]$ & 3.41 & 2.87 & 3.10 & 3.12 & 0.27 \\
\hline
\end{tabular}

Table 7.10: Values determined for the stochastic and constant terms using different sets of transverse weights derived without beam energy constraints from $120 \mathrm{GeV}, 200 \mathrm{GeV}$ pion data, and the average of weights from $120,150,200 \mathrm{GeV}$ pion data.

hadron energy resolution with a stochastic term $a=72.6 \pm 1.08($ sys $) \pm 0.40($ stat $) \% \cdot \sqrt{\mathrm{GeV}}$ and a constant term $b=2.87 \pm 0.27($ sys $) \pm 0.007($ stat $) \%$. Here, the systematic error for the stochastic (constant) term was estimated using the RMS of the stochastic (constant) terms shown in Table 7.10 using all derived transverse weights.

As with the baseline approach, a set of weights constrained with the beam energy was also investigated. In order to constrain the transverse weights, a set of flat weights was applied after the energy was reconstructed using the transverse weight. This was done using Equations 7.8 and 7.9. Results from the beam constrained minimization using beam energies of $100,120,150$, and $200 \mathrm{GeV}$ to the transverse weights obtained with $200 \mathrm{GeV}$ pion data are presented in Table 7.11. From this table it can seen that the flat weights are energy dependent. The $\alpha_{1}$ term decreases with energy while the $\alpha_{3}$ term increases with energy. These systematic variations are believed to be due to the difference in the fractional amount of energy deposited in the FCal modules.

The results from the combination of transverse weights and beam energy constraining flat weights are presented in Table 7.12. Using the combination of transverse weights and 

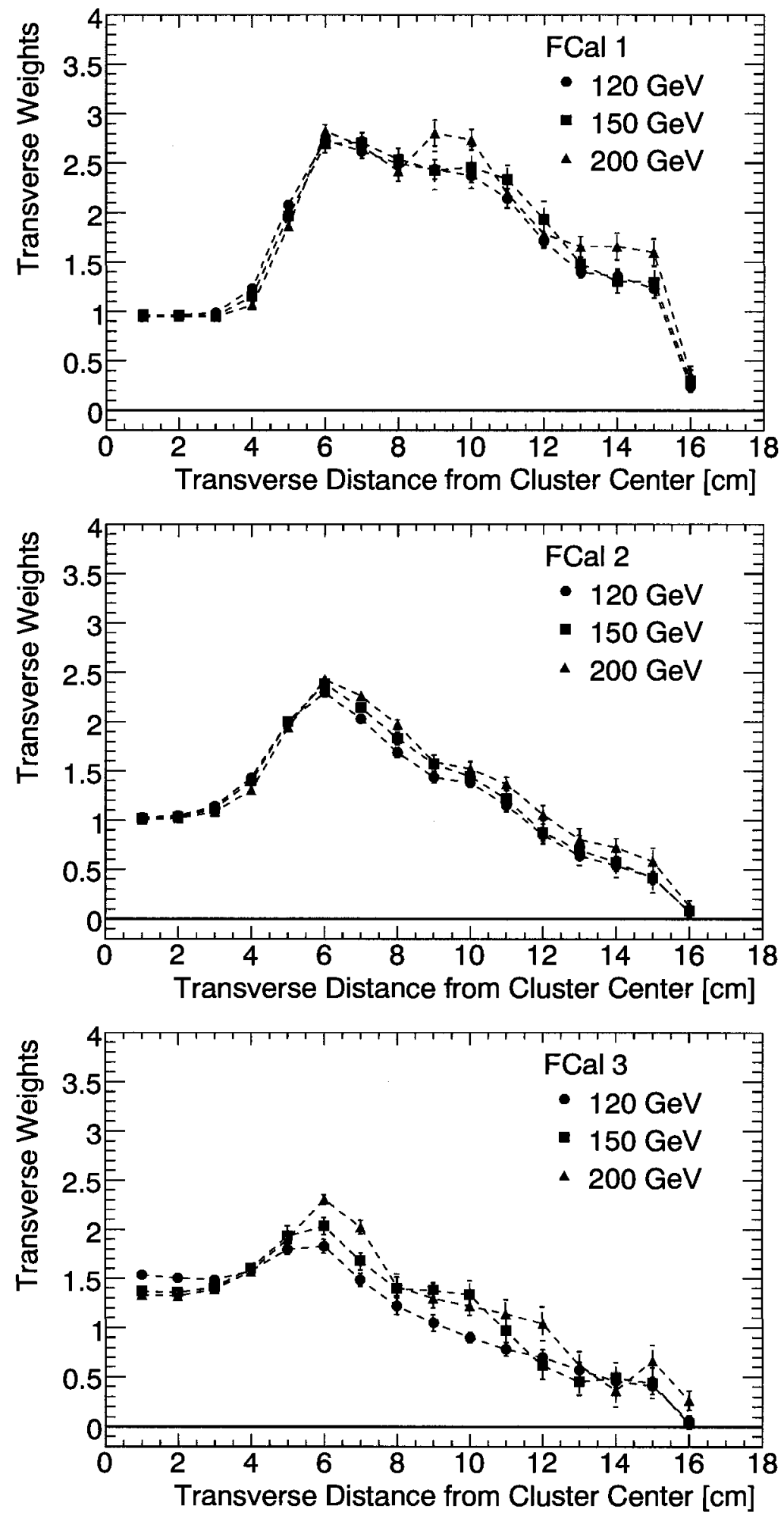

Figure 7.9: Transverse weights for all three FCal modules derived from 120, 150, and 200 $\mathrm{GeV}$ energy hadrons. 


\begin{tabular}{|c|c|c|c|c|c|c|}
\hline \multirow{2}{*}{$\begin{array}{c}\text { Calibration } \\
\text { Weights }\end{array}$} & \multicolumn{4}{|c|}{ Energy [GeV] } & \multirow{2}{*}{ Mean } & \multirow{2}{*}{$\begin{array}{c}\text { RMS } \\
{[\%]} \\
\end{array}$} \\
\hline & 100 & 120 & 150 & 200 & & \\
\hline$\alpha_{1}$ & 1.020 & 1.019 & 1.014 & 1.009 & 1.016 & 0.493 \\
\hline$\alpha_{2}$ & 1.022 & 1.025 & 1.024 & 1.018 & 1.022 & 0.316 \\
\hline$\alpha_{3}$ & 0.968 & 0.985 & 0.983 & 0.985 & 0.980 & 0.826 \\
\hline
\end{tabular}

Table 7.11: Flat weights that are used to give beam energy constraints to the transverse weights obtained with $200 \mathrm{GeV}$ pion data.

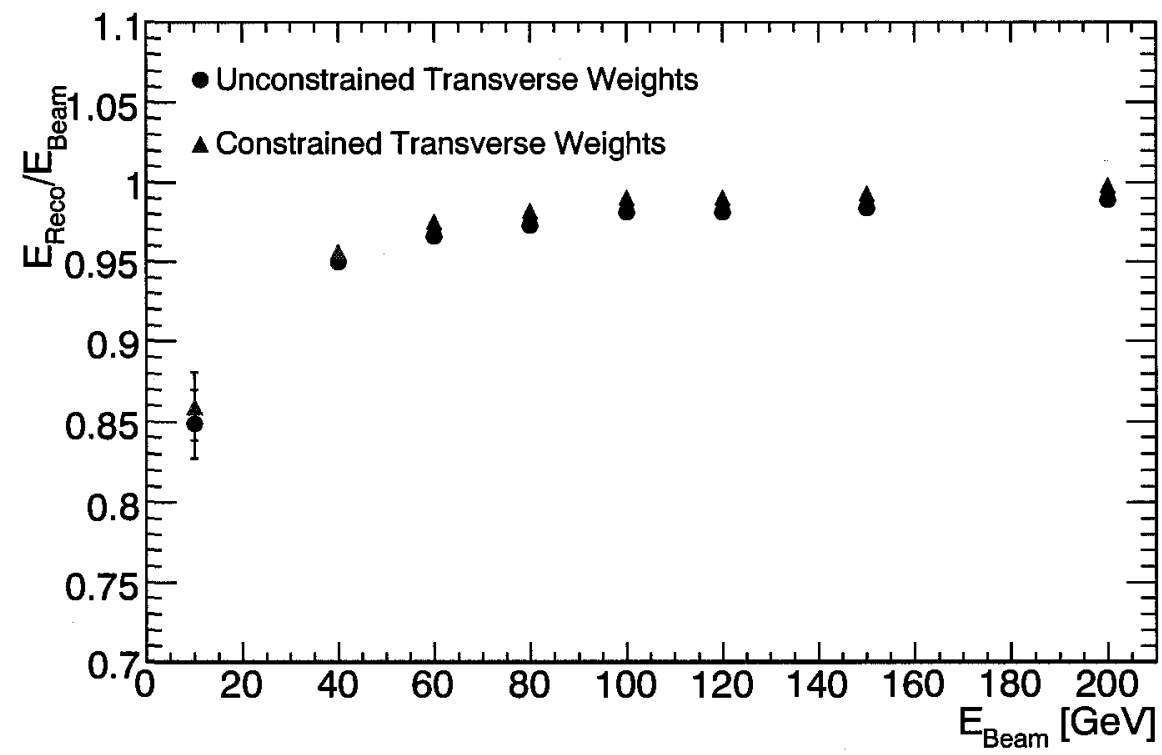

Figure 7.10: Comparison of the energy response using transverse weights derived with and without beam constraints. The flat weights used to constrain the transverse weights were derived from $200 \mathrm{GeV}$ pion data. The ratio between the reconstructed energy and the beam energy, $E_{\text {Reco }} / E_{\text {Beam }}$, was used to quantify the beam response.

\begin{tabular}{|c|c|c|c|c|c|c|}
\hline \multirow{2}{*}{$\begin{array}{l}\text { Resolution } \\
\text { Terms }\end{array}$} & \multicolumn{4}{|c|}{ Energy used to Derived Weights [GeV] } & \multirow{2}{*}{ Mean } & \multirow{2}{*}{ RMS } \\
\hline & 100 & 120 & 150 & 200 & & \\
\hline Stochastic $[\% \cdot \sqrt{\mathrm{GeV}}]$ & 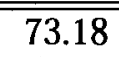 & 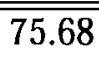 & $\overline{72.58}$ & 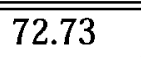 & $\overline{73.54}$ & $\overline{1.45}$ \\
\hline Constant [\%] & 2.39 & 1.94 & 2.90 & 2.85 & 2.52 & 0.45 \\
\hline
\end{tabular}

Table 7.12: Values determined for the stochastic and constant terms using transverse weights and beam energy constraints. The flat weights used to constrain the transverse weights were derived using $100,120,150$, and $200 \mathrm{GeV}$ pion data and the transverse weights were derived using $200 \mathrm{GeV}$ pion data. 
beam energy constraining flat weights derived from the $200 \mathrm{GeV}$ pion data provides a hadron energy resolution with a stochastic term of $a=72.7 \pm 1.45$ (sys) \pm 0.38 (stat) \% . $\sqrt{\mathrm{GeV}}$ and a constant term of $b=2.85 \pm 0.45($ sys $) \pm 0.007$ (stat)\%. Here, the systematic error for the stochastic (constant) term was estimated using the RMS of the stochastic (constant) terms shown in Table 7.12 using all derived transverse weights and beam energy constraints.

From the results presented in Tables 7.9 and 7.12 it is clear that the use of the transverse weights significantly improves the energy resolution for hadrons. In addition, the energy response using transverse weights is significantly more uniform than when flat weights are used, throughout the full energy range, as is shown in Figure 7.11. The energy resolution is also systematically better for the transverse weights, as shown in Figure 7.12. Moreover, at the $200 \mathrm{GeV}$ pion beam energy, the energy resolution using transverse weights is a factor of 1.6 smaller than using the flat weights. Finally, the $e / \pi$ response can be inferred from Figure 7.11 given that the electron response has been shown to be linear to within $1 \%$. Using the flat weights the $e / \pi$ varies from 1.33 to 1.00 between $10 \mathrm{GeV}$ and $200 \mathrm{GeV}$ while with the transverse weights the ratio only varies from 1.15 to 1.00 , providing a more uniform response. Note that the fitting procedure equalizes the electron and pion response at $200 \mathrm{GeV}$.

\subsection{Conclusion}

In this part of the thesis studies on the electron and hadron performance were conducted. The main goals of the electron study were to determine the linearity and resolution for electrons as measured in the FCal 1 module. The linearity measurement provided a direct comparison with a simulated model of the readout electronics, this model will provide the initial electromagnetic response for the FCal modules during the early stages of ATLAS data taking. The measured electromagnetic response was $12.14 \pm 0.06($ sys $) \pm 0.002($ stat $)$ 


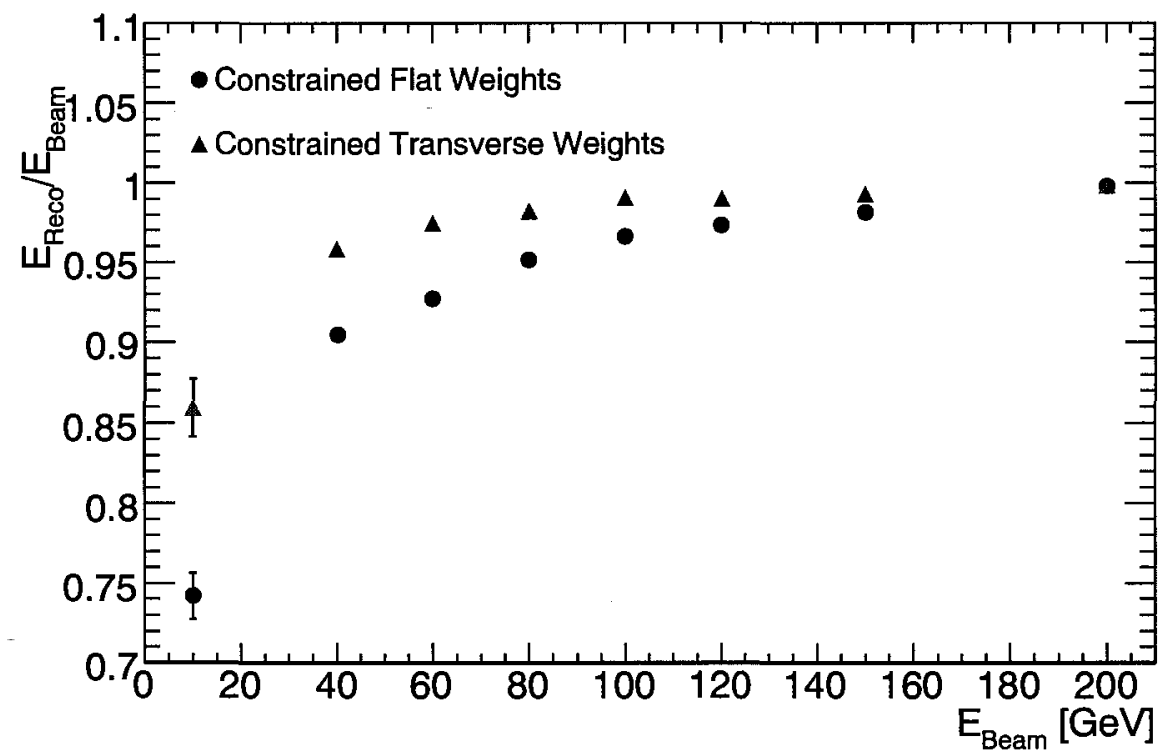

Figure 7.11: Comparison of the energy response between beam constrained flat and transverse weights. The ratio between the reconstructed energy and the beam energy, $E_{\text {Reco }} / E_{\text {Beam }}$, was used to quantify the beam response.

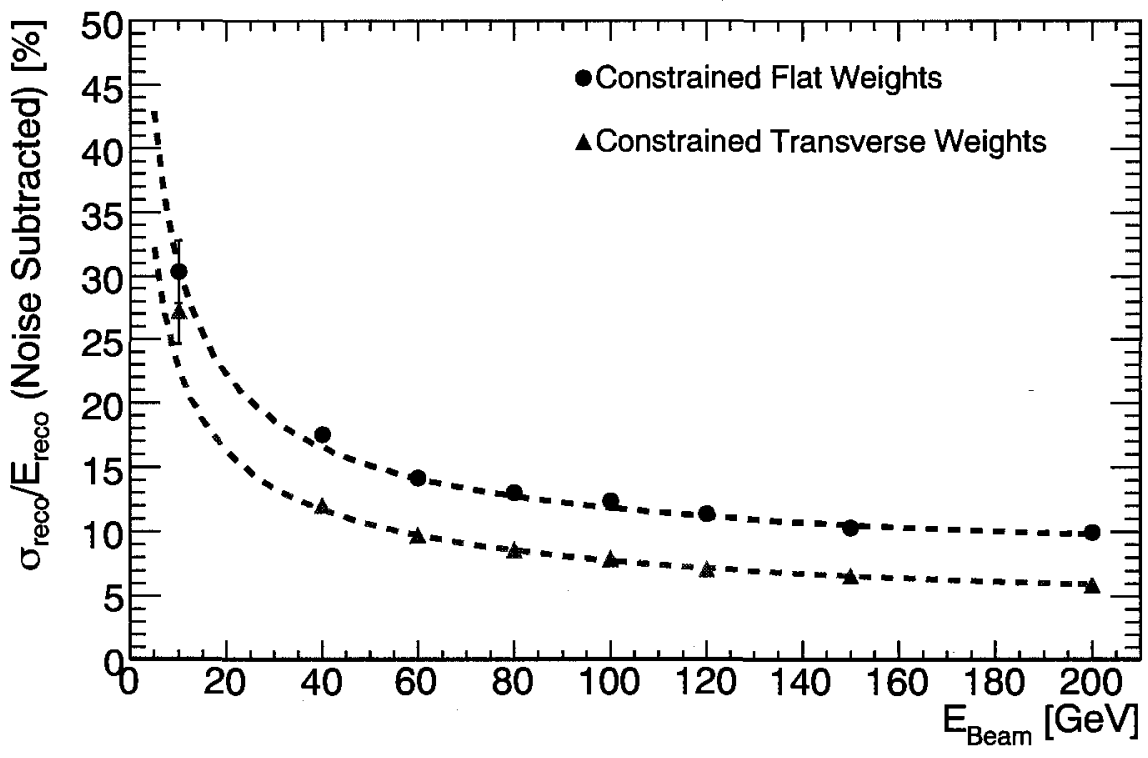

Figure 7.12: Comparison of the energy resolution between beam constrained flat and transverse weights. 
$\mathrm{ADC} / \mathrm{GeV}$ which is in good agreement with the predicted value of $12 \mathrm{ADC} / \mathrm{GeV}$ from the simulation. In addition, the measured energy resolution for electrons was parameterized as $\sigma_{E} / E=(27.96 \pm 0.82) \% / \sqrt{E_{b}} \oplus(3.48 \pm 0.13) \%$ which is consistent with previously published FCal test beam results $[23,26]$.

The ATLAS FCal performance requirement for jets is a transverse energy, $E_{T}$, resolution of $\Delta E_{T} / E_{T}<10 \%$ for $E_{T}>25 \mathrm{GeV}$. Jets below this minimum $E_{T}$ of $25 \mathrm{GeV}$ are expected to be lost due to pile-up. In order to measure the hadron performance, pion data collected during the 2003 test beam was used. As such, two calibration schemes were investigated: flat weight and transverse weights. The latter calibration technique was developed to take advantage of the unique geometry of the FCal detector. The energy resolutions for hadrons using beam constrained flat weights and transverse weights were parameterized as $\sigma_{E} / E=(98.25 \pm 1.85) \% / \sqrt{E_{b}} \oplus(7.13 \pm 0.10) \%$ and $\sigma_{E} / E=$ $(72.7 \pm 1.5) \% / \sqrt{E_{b}} \oplus(2.85 \pm 0.28) \%$, respectively. These results show that the transverse weights reduce the stochastic term from $(98.25 \pm 1.85) \%$ to $(72.7 \pm 1.5) \%$ and the constant term from $(7.13 \pm 0.10) \%$ to $(2.85 \pm 0.45) \%$. For a $200 \mathrm{GeV}$ pion the energy resolutions are $\sim 10 \%$ using flat weights which is close to the performance goals and $\sim 6 \%$ using transverse weights which exceeds the performance requirements. In addition, the transverse weights provided a uniform energy response to within $3 \%$ for energies greater than 40 $\mathrm{GeV}$. 


\section{Part II}

\section{ATLAS Sensitivity to an Invisible \\ Higgs via Vector Boson Fusion}




\section{Chapter 8}

\section{Introduction}

The primary objective of the LHC is to investigate the mechanism of electroweak symmetry breaking, which can be explained by the Higgs mechanism. In order to gain confidence in the Standard Model (SM), measurements are performed to test and validate the model. It is also important to investigate alternate mechanisms which could influence our understanding of how electroweak symmetry breaking works. Subjecting the SM to numerous tests gives us confidence that this model provides a proper explanation of the fundamental particles of nature and the interaction between them.

The search for the invisible Higgs is inherently a search for physics beyond the SM. This can be understood because in the SM the Higgs invisible decay width is very small. For example, a Higgs of mass $140 \mathrm{GeV}$ has an invisible decay width of $\sim 8 \mathrm{MeV}$, which will be very difficult to observe. Therefore, since the invisible decay width of the SM Higgs is small, an increase in the width beyond the SM value is an indication of new physics. In this thesis, results on the sensitivity of the ATLAS experiment to new interactions enhancing invisible Higgs decay are investigated.

In this chapter, a brief review of the SM, focusing on the Higgs boson, is presented in Sections 8.1 and 8.2. Afterwards, a summary of various models providing an enhanced 


\begin{tabular}{|c|c|c|c|}
\hline Flavor & Charge & Mass [GeV] & Force \\
\hline \hline$\gamma$ & 0 & 0 & Electromagnetic \\
\hline$Z^{0}$ & 0 & 91.2 & Weak \\
$W^{ \pm}$ & \pm 1 & 80.4 & \\
\hline g (8 gluons) & 0 & 0 & Strong \\
\hline
\end{tabular}

Table 8.1: Properties of Standard Model Gauge Bosons.

invisible Higgs decay are presented in Section 8.3. Concluding, the existing limits for invisible Higgs decay are presented in Section 8.4.

\subsection{The Standard Model and the Higgs boson}

The SM of particle physics is a model of fundamental particles and their interactions. There are two types of fundamental particles in this theory, they are: spin 1/2 fermions and integer spin bosons. Matter is composed of fermions, which are classified as either quarks or leptons. In contrast, bosons are particles which mediate the interactions between the fermions. The SM is composed of two theories: the electroweak theory proposed by Glashow, Weinberg, and Salam [27-29] and Quantum ChromoDynamics (QCD). The interactions between particles are associated with the local symmetries as defined by the $S U(3)_{c} \otimes S U(2)_{L} \otimes U(1)_{Y}$ gauge group. The QCD theory, $S U(3)_{c}$, describes the interaction between quarks through the strong force via color (c) charge and is mediated by eight massless gluons. The electroweak theory, $S U(2)_{L} \otimes U(1)_{Y}$, incorporates the electromagnetic and weak interactions between fermions and is mediated by four gauge bosons. These bosons are the photons of electromagnetism and the $\mathrm{W}^{ \pm}$and $\mathrm{Z}^{0}$ bosons of the weak force.

In the SM, there are six leptons and six quarks which fall into three families of identical structure as listed in Table 8.2. The only differences between the generations are the mass of the fermions and their flavor number. Fermions exist in either a left or righthanded chirality. Only the left-handed fermions can interact via the weak force. This left- 


\begin{tabular}{|c|c|c|c|c|c|c|}
\hline \multirow{2}{*}{ Generations } & \multicolumn{3}{|c|}{ Leptons } & \multicolumn{3}{c|}{ Quarks } \\
\cline { 2 - 7 } & Flavor & Charge & Mass [GeV] & Flavor & Charge & Mass [GeV] \\
\hline \hline \multirow{2}{*}{$1^{\text {st }}$} & $\nu_{e}$ & 0 & $<3 \times 10^{-9}$ & $\mathrm{u}$ & $+\frac{2}{3}$ & $3 \times 10^{-3}$ \\
& $\mathrm{e}$ & -1 & $5.11 \times 10^{-4}$ & $\mathrm{~d}$ & $-\frac{1}{3}$ & $7 \times 10^{-3}$ \\
\hline \multirow{2}{*}{$2^{n d}$} & $\nu_{\mu}$ & 0 & $<1.9 \times 10^{-4}$ & $\mathrm{c}$ & $+\frac{2}{3}$ & 1.2 \\
& $\mu$ & -1 & 0.106 & $\mathrm{~s}$ & $-\frac{1}{3}$ & 0.1 \\
\hline \multirow{2}{*}{$3^{r d}$} & $\nu_{\tau}$ & 0 & $<18.2 \times 10^{-3}$ & $\mathrm{t}$ & $+\frac{2}{3}$ & 174 \\
& $\tau$ & -1 & 1.777 & $\mathrm{~b}$ & $-\frac{1}{3}$ & 4.2 \\
\hline \hline
\end{tabular}

Table 8.2: Properties of Standard Model fermions classified in three generations.

right handed asymmetry in the theory was guided by experimental results. Left-handed fermions form weak isospin doublets while right-handed fermions exist as weak isospin singlets. Equation 8.1 illustrates the fermion chirality for the first generation leptons, that is, the electron and its associated neutrino.

$$
L_{e}=\left(\begin{array}{c}
\nu_{e} \\
e
\end{array}\right)_{L}, R_{e}=e_{R}
$$

In order to introduce mass in the SM for the fermions and bosons, the Higgs mechanism is used. This is done by adding a complex scalar doublet with a hypercharge +1 . Although the SM agrees very well with most experimental measurements, there are several compelling experimental observations which suggest the need for physics beyond the SM, such as neutrino mixing, dark matter, and baryon asymmetry. None of these observations can be accommodated within the SM.

\subsection{Phenomenology of the Standard Model Higgs}

In this section, the theoretical and experimental constraints on the phenomenology of the SM Higgs are reviewed. Afterwards, a summary of the Higgs production and branching ratio is discussed. 


\subsubsection{Constraints on the Standard Model Higgs Mass}

Although the Higgs boson has yet to be discovered, there are several theoretical and experimental constraints on the SM Higgs mass.

There are theoretical constraints on the mass of the SM Higgs such as the triviality bound and vacuum stability. The triviality bound can be understood by investigating the quartic coupling term in the Higgs potential, that is $\lambda=M_{h}^{2} / 2 v^{2}$. Here, $M_{h}$ is the mass of the SM Higgs and $v$ is the vacuum expectation value. The running of $\lambda(Q)$ via self interaction at some effective energy scale, Q, is given in Equation 8.2 [30].

$$
\lambda(Q)=\frac{\lambda(v)}{1-\frac{3 \lambda(v)}{4 \pi^{2}} \log \left(\frac{Q^{2}}{v^{2}}\right)}
$$

If one assumes that there is no new physics, then $\lambda(Q)$ should be valid for all $\mathrm{Q}$ values. For $\lambda(Q)$ to be finite as $Q \rightarrow \infty$, then $\lambda(Q) \rightarrow 0$. However, if $\lambda=0$, then there is no selfcoupling, in other words, a trivial theory. Therefore, in order to determine the triviality bound, $\lambda$ is required to be finite and $1 / \lambda(Q)>0$. This provides an approximate upper bound on the Higgs mass as shown in Equation 8.3 [31].

$$
M_{h}^{2}<\frac{8 \pi^{2} v^{2}}{3 \log \left(Q^{2} / v^{2}\right)}
$$

If there is no new physics expected until $10^{16} \mathrm{GeV}$, then the upper bound on the SM Higgs would be $\sim 160 \mathrm{GeV}$. Conversely, the triviality bound argument tells us that if the SM Higgs is heavy then the SM is no longer valid which is suggestive of new physics. In this example, the coupling to the fermions and gauge bosons has been neglected. However, the argument given above provides a reasonable approximation.

In addition to the triviality bound, vacuum stability provides a lower limit to the SM Higgs mass. This requires spontaneous symmetry breaking to actually occur, i.e. the 
Higgs potential has a minimum at the vacuum expectation value, as shown in Equation 8.4.

$$
V(v)<V(0)
$$

This is also equivalent to requiring $\lambda(Q)$ to be greater than zero. If the SM is valid up to scales of order $10^{16} \mathrm{GeV}$, the lower limit for the Higgs mass based on this argument would be between approximately $130-170 \mathrm{GeV}$.

In addition to these theoretical limits, experimental data provide constraints on both the lower and upper limits of the Higgs mass. A direct LEP search for the Higgs boson imposes a lower limit of $114.4 \mathrm{GeV}$ at $95 \%$ confidence level [32]. In addition, experimental data can put constraints on the upper limit of the SM Higgs mass by considering the impact of radiative corrections on electroweak precision measurements. At the one loop level, radiative corrections which include the SM Higgs mass enter logarithmically into the calculations. Therefore, the presence of the SM Higgs can be indirectly inferred by looking for radiative correction effects using electroweak precision data. Figure 8.1 shows results from the LEP Electroweak Working Group on the global fit of the electroweak data as a function of Higgs mass. From this figure, the favored mass is located at $76_{-24}^{+33} \mathrm{GeV}$, which has already been excluded by direct searches at LEP [33]. However, the 95\% confidence level upper limit from the electroweak data is $\sim 144 \mathrm{GeV}$.

Figure 8.2 shows the theoretical and experimental constraints on the Higgs mass as a function of the effective energy scale. There are two complementary theoretical and experimental conditions for the upper and lower bounds. For lower effective energy scales the experimental constraint provides the strongest limits on the Higgs mass. The electroweak precision measurement (shaded in pink) provides the upper bound and the direct search from LEP (shaded in yellow) provides the lower bound. As the effective energy scale increases, the theoretical limits on the lower bound provides the strongest limit. It is only at 


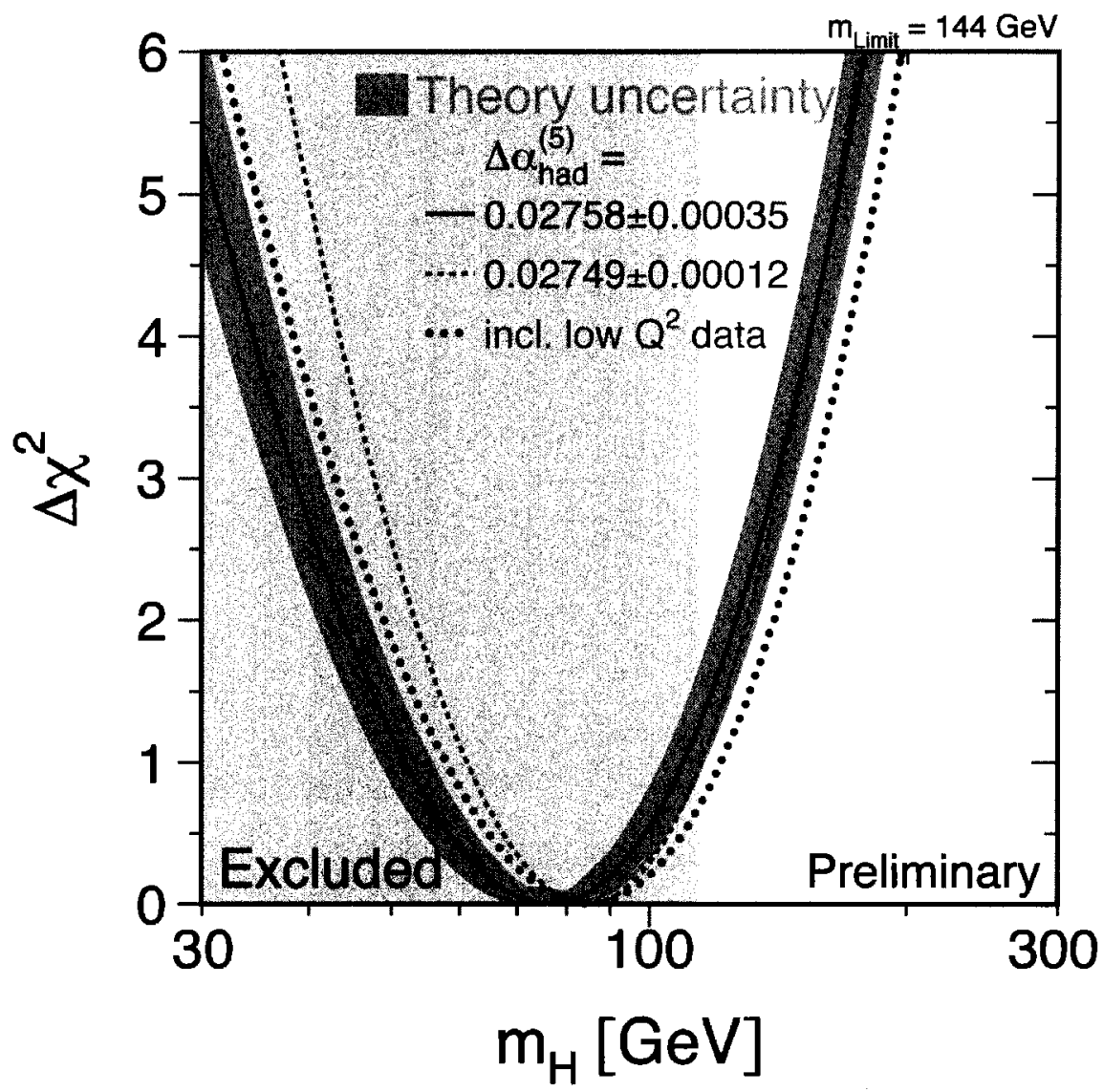

Figure 8.1: Electroweak precision data as a function of Higgs mass [33]. The solid line is the result from the fit and the blue band is the theoretical uncertainty for missing higher order corrections. The yellow band is the excluded region from direct searches at LEP. Here $\Delta \chi^{2}=\chi^{2}-\chi_{\min }^{2}$. 
the very high effective energy scales that the theoretical upper bound provides a tighter constraint on the Higgs mass. From this figure, a Higgs mass between approximately $115-190 \mathrm{GeV}$ is clearly favored.

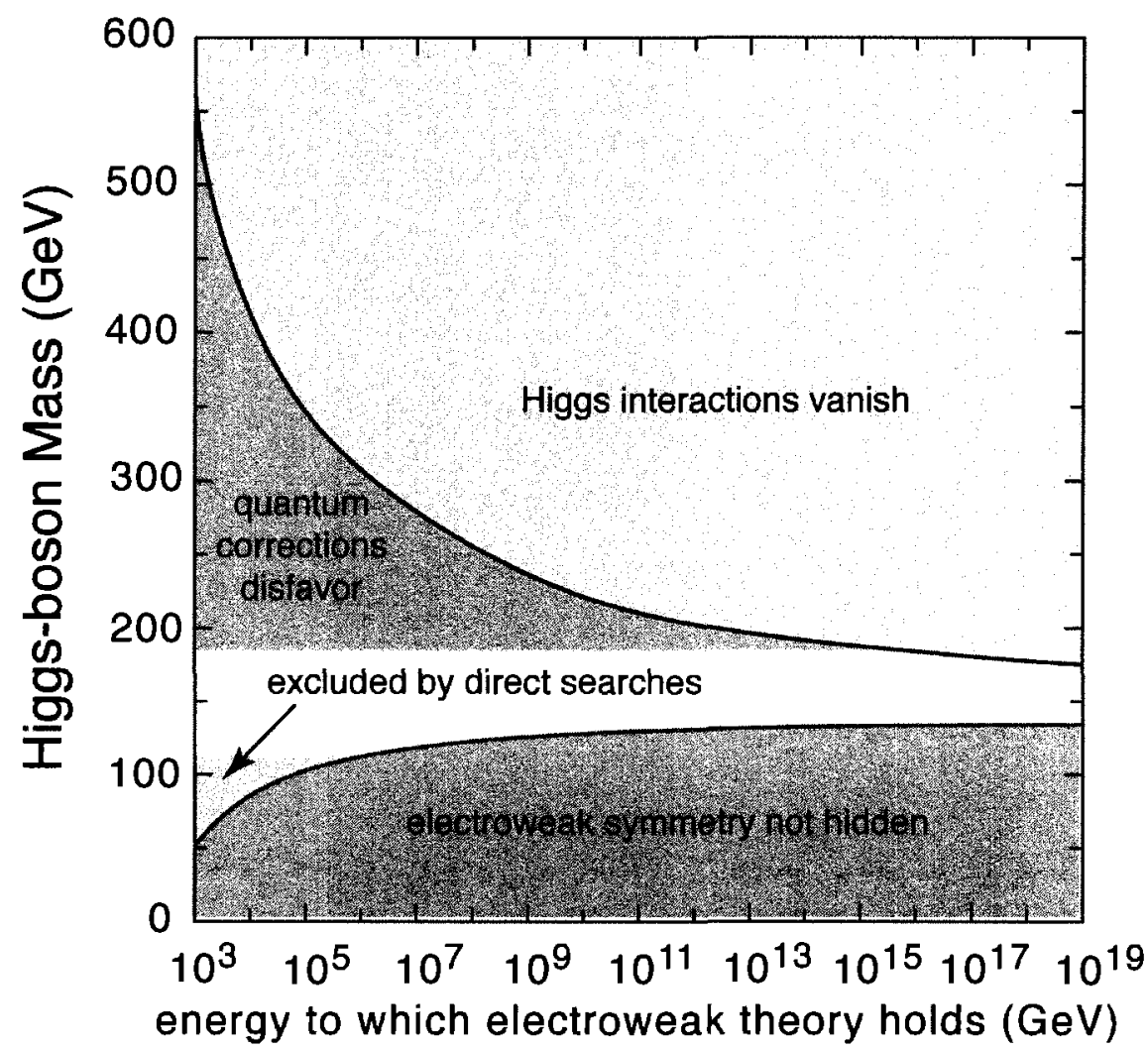

Figure 8.2: Constraints on the Higgs mass as a function of the effective energy scale [34]. The upper bound is the constraint provided by the triviality condition. The lower bound is provided by the vacuum stability condition, that is, the breaking of electroweak symmetry. In addition, constraints from direct searches and electroweak precision measurements are shown. The white band indicates the favored Higgs mass range between approximately $115-190 \mathrm{GeV}$.

\subsubsection{Higgs Production and Branching Ratio at the LHC}

There are four production modes for the SM Higgs at the LHC and they are presented in Figures 8.3 and 8.4. The dominant production mode is the gluon-gluon fusion, $g g \rightarrow H$, which has a rate that is more than an order of magnitude larger than any other production mode. It occurs via a triangle loop, as shown in Figure $8.4(\mathrm{a})$ which is dominated by 
the top quark since the Yukawa coupling is proportional to the fermion mass. Vector Boson Fusion (VBF) has the second largest production rate as shown in Figure 8.3. This production mode has two well separated jets which tend to be predominantly located in opposite hemispheres. The VBF process is an electroweak process, as shown in Figure 8.4(b). As a consequence, there is little activity between these two jets due to its unique color flow. In addition to these production modes, there are several associated production modes with similar cross-sections. Feynman diagrams for two such modes, ZH and $t \bar{t}$, are shown in Figures 8.4(c) and 8.4(d). These production channels have small cross-sections compared to the total Higgs cross-section, but can provide a clear signature. Figure 8.5 shows the branching ratios for the decay of a SM Higgs as a function of the Higgs mass. At low Higgs mass, the $b \bar{b}$ decay mode dominates since the Yukawa coupling is proportional to the fermion mass squared. The second largest low mass Higgs decay mode is the $\tau \tau$ which is one of the primary discovery channels at the ATLAS detector. Once the Higgs mass approaches the WW and ZZ mass threshold, it predominantly decays into these modes. For Higgs masses above $\sim 350 \mathrm{GeV}$, the $t \bar{t}$ decay mode provides a significant contribution.

\subsection{Physics Beyond the Standard Model and the Invisible Higgs}

Although the SM is very successful in describing physics in the region of energy that has been experimentally tested, it is believed it will fail at higher energies. Based on unitarity arguments, if the Higgs is not found then the SM will fail at the TeV scale. Therefore, new models are suggested for studies at the $\mathrm{TeV}$ scale which might include new observable physics processes. However, even if the Higgs boson is discovered, there are several aspects of the Standard Model which are somewhat unsatisfactory. Apart from the obvious inability to account for gravity, there are some puzzling questions which the Standard Model does not answer, such as:

1. Why is the weak force so much stronger than gravity? (hierarchy problem) 


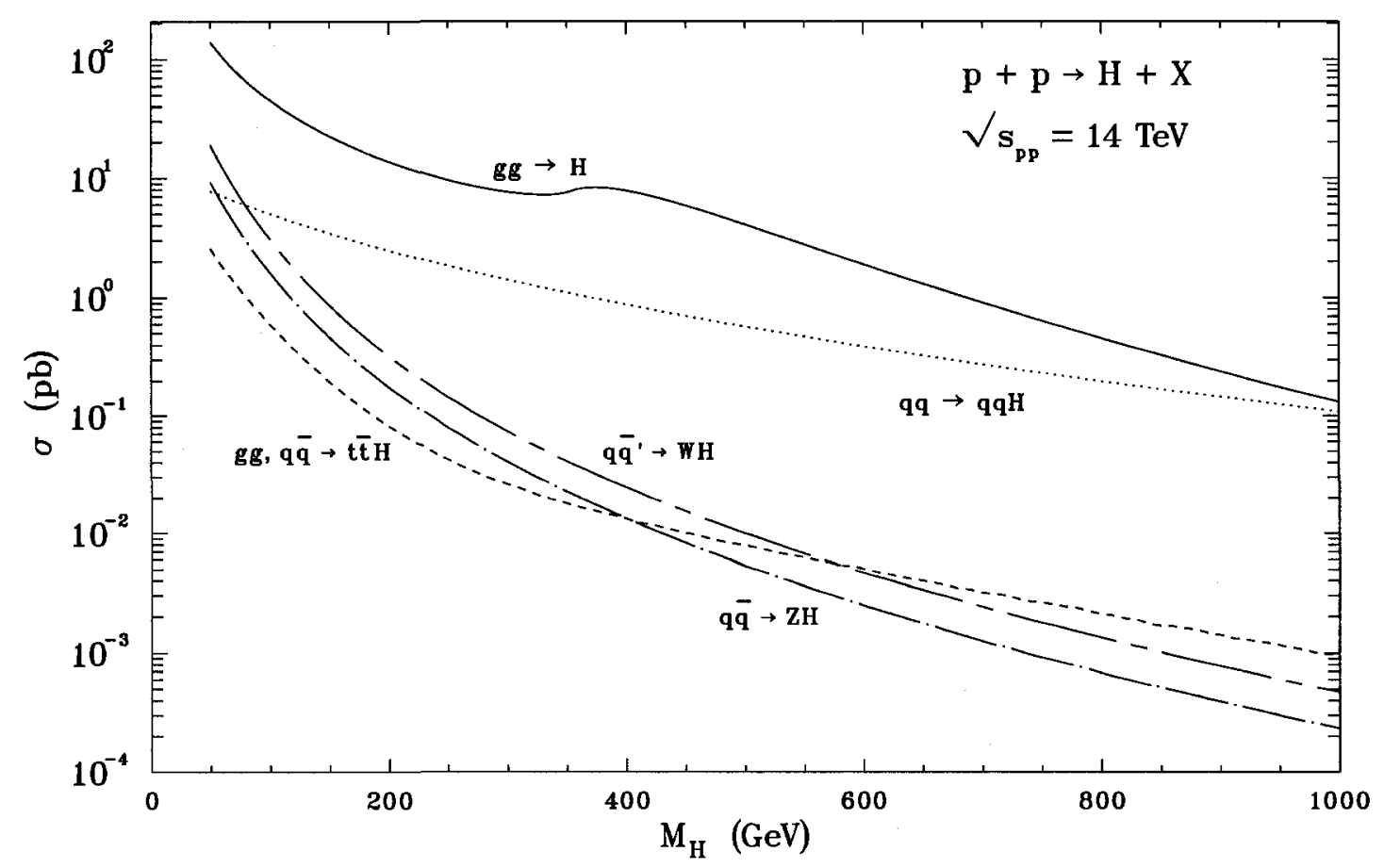

Figure 8.3: Higgs production cross-section in the Standard Model [35].

2. Why is there baryon anti-baryon asymmetry?

3. Why is there more than one fermion generation?

Several extensions to the Standard Model try to address these questions, such as SUperSYmmetry (SUSY) and Extra Dimension (ED) models. In order to provide a complete study of the Higgs sector, the ATLAS collaboration will need to search for these extensions of the Standard Model. One consequence of these models is the possibility that the Higgs can decay into particles which do not produce a signal in the experiment, which is generally referred to as an "Invisible Higgs". The Standard Model Higgs has a very small invisible branching ratio, therefore, an observed excess of invisible events would indicate a non-Standard Model Higgs. It should be noted that in an invisible Higgs analysis, the Higgs mass is not reconstructed. Therefore, evidence for an invisible Higgs is determined by the excess number of events from the expected number of background events. There are several possible models which suggest that the Higgs can predominantly decay invisibly, such as: invisible decay to neutralinos in SUSY models [36, 37], heavy photons 


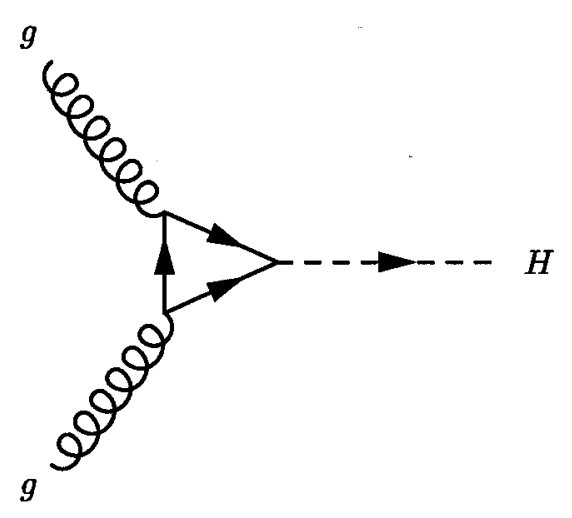

(a) Gluon fusion

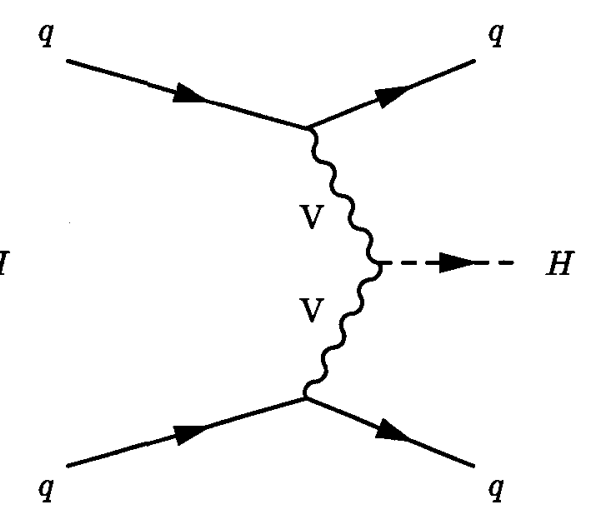

(b) Vector boson fusion

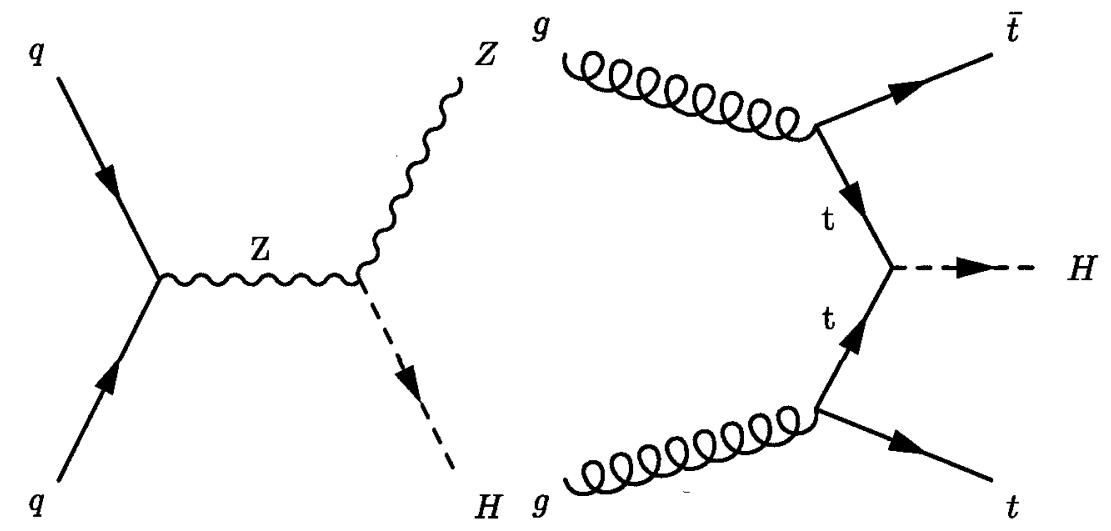

(c) Associated to the $\mathrm{Z}$ boson

(d) Associated to the $t \bar{t}$ pair

Figure 8.4: Feynman diagrams for the Higgs production modes.

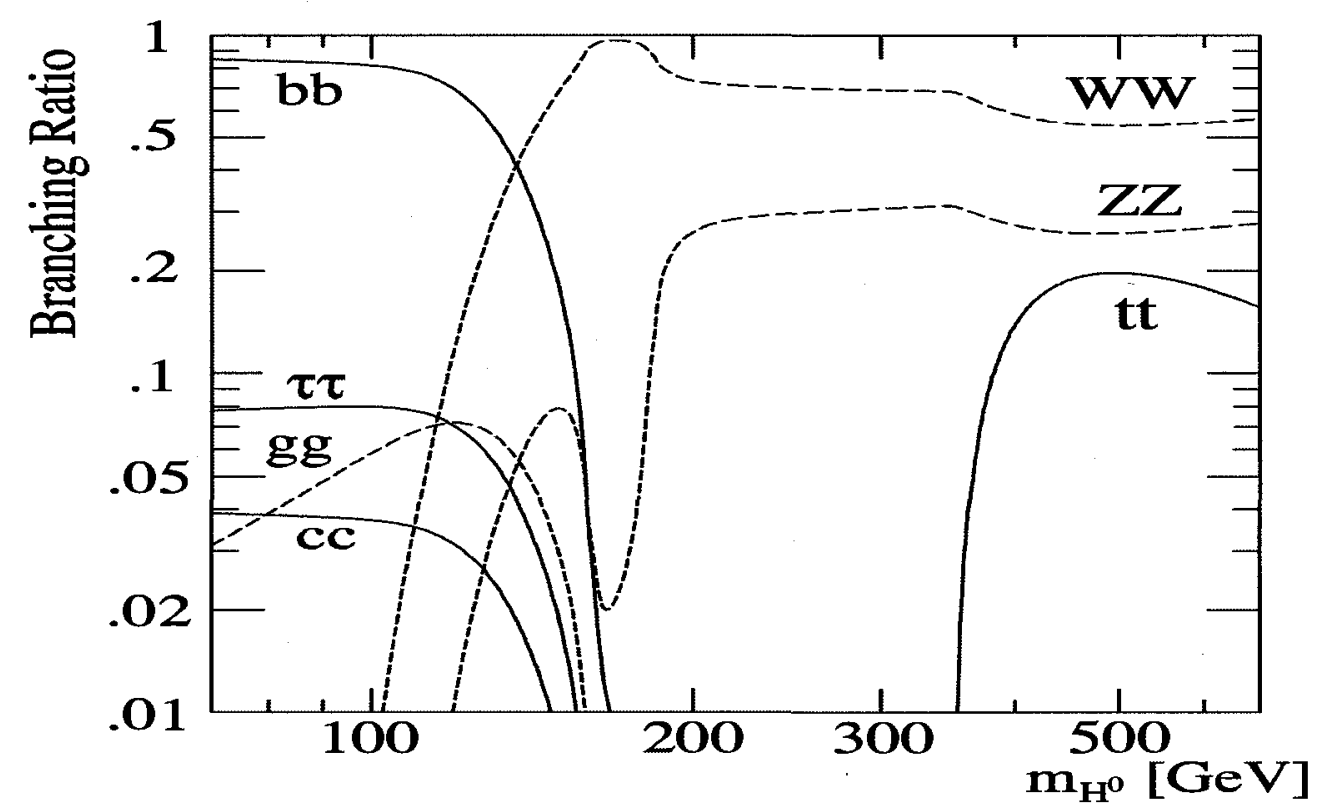

Figure 8.5: The Standard Model Higgs branching ratio as a function of Higgs mass [19]. 
in the littlest Higgs model [38], phions in steathly Higgs [39, 40], and Kaluza-Klein (KK) graviscalar states in extra dimension $[41,42]$. This is not intended to be an exhaustive list, but rather an indication of the numerous possible models which can explain an invisible Higgs decay. The following is a brief summary of two models which produce a Higgs-like particle decaying invisibly.

Massive 4th Generation Neutrinos In this model, the existence of massive $4^{\text {th }}$ generation neutrinos is considered. This model provides the simple framework to introduce a dominant invisible Higgs decay mode [43]. From the measurements of the $\mathrm{Z}$ boson width, the $95 \%$ confidence lower limit on the mass of these neutrinos is $46.7 \pm 0.2 \mathrm{GeV}$ [44]. In addition, massive $4^{\text {th }}$ generation neutrinos are compatible with electroweak precision data providing their mass is $\sim 50 \mathrm{GeV}$ [45]. However, the $4^{\text {th }}$ generation neutrinos are also constrained from above with an upper mass limit around the W boson mass [43]. The branching ratio for a Higgs decaying to massive $4^{\text {th }}$ generation neutrinos is given in Equation 8.5 [43].

$$
\Gamma\left(h \rightarrow \nu_{4} \nu_{4}\right)=\frac{\sqrt{2}}{8 \pi} G_{F} m_{\nu_{4}}^{2} m_{h}\left(1-\frac{4 m_{\nu_{4}}^{2}}{m_{h}^{2}}\right)^{3 / 2}
$$

Here, $G_{F}$ is the Fermi constant, $m_{h}$ is the Higgs mass and $m_{\nu_{4}}$ is the mass of the $4^{\text {th }}$ generation neutrino. The branching ratio for an invisible Higgs as a function of Higgs mass assuming a $4^{\text {th }}$ generation neutrino mass of $50 \mathrm{GeV}$ is presented in Figure 8.6, which illustrates the dominant invisible branching ratio.

Extra Dimension In the Arkani-Hamed-Dimopoulos-Dvali (ADD) model [46] the Higgs can decay into the closest KK graviscalar levels. These graviscalars interact weakly because they propagate in the bulk, where as the SM particles are confined to the 4-dimensional brane. If the Higgs boson decays into these graviscalars, the branching ratio would be given by Equation 8.6 [47].

$$
\Gamma(h \rightarrow \mathcal{G})=2 \pi k^{2} \xi^{2} v^{2} \frac{m_{h}^{1+\delta}}{M_{D}^{2+\delta}} \frac{2 \pi^{\delta / 2}}{\Gamma(\delta / 2)}
$$




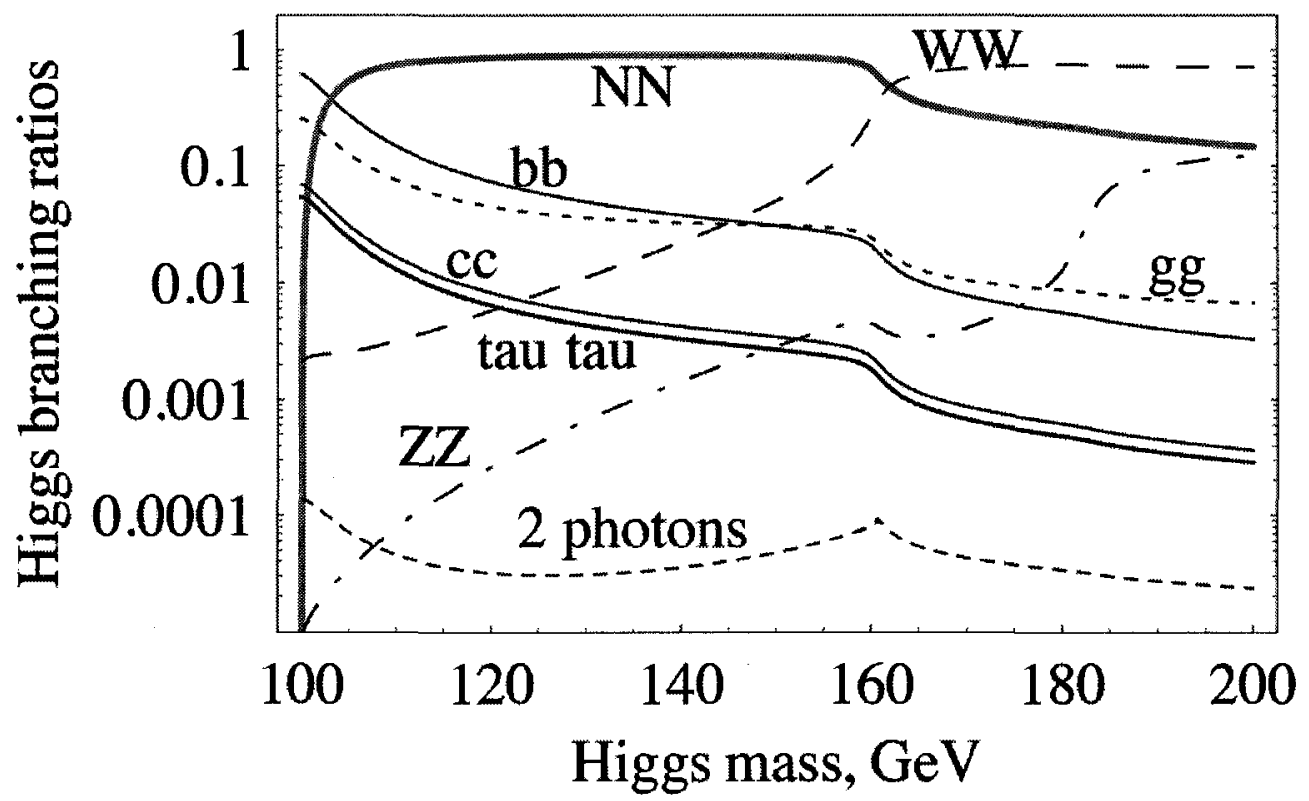

Figure 8.6: Invisible Higgs branching ratio, $\Gamma\left(h \rightarrow \nu_{4} \nu_{4}\right)$, as a function of Higgs mass assuming $m_{\nu_{4}}=50 \mathrm{GeV}$ [43] for a model that includes massive $4^{\text {th }}$ generation neutrinos. The line associated with the label NN is the $h \rightarrow \nu_{4} \nu_{4}$ branching ratio.

Here $k \equiv 3(\delta-1) / \delta+2$ where $\delta$ is the number of extra dimensions, $\xi$ is a dimensionless mixing parameter, and $v$ is the vacuum expectation value. In addition, $m_{h}$ is the Higgs mass and $M_{D}$ is the $(4+\delta)$-dimensional Planck scale. The branching ratio of the Higgs to an invisible channel, as a function of $\xi$ with two extra compactified dimensions for various $M_{D}$ energy scales, is presented in Figure 8.7. This figure clearly illustrates a dominant invisible branching ratio for a $120 \mathrm{GeV}$ Higgs mass assuming two additional compactified extra dimensions and a $(4+\delta)$-dimensional Planck scale of $1.5 \mathrm{TeV}$ for $\xi$ between 0 and 1 .

\subsection{Status of Invisible Higgs Search}

Presented in this section are the results of previous studies on the invisible Higgs. These studies include the results from LEP and simulations using the ATLAS detector. The sensitivity for observing an invisible Higgs used for these studies is given by the variable 


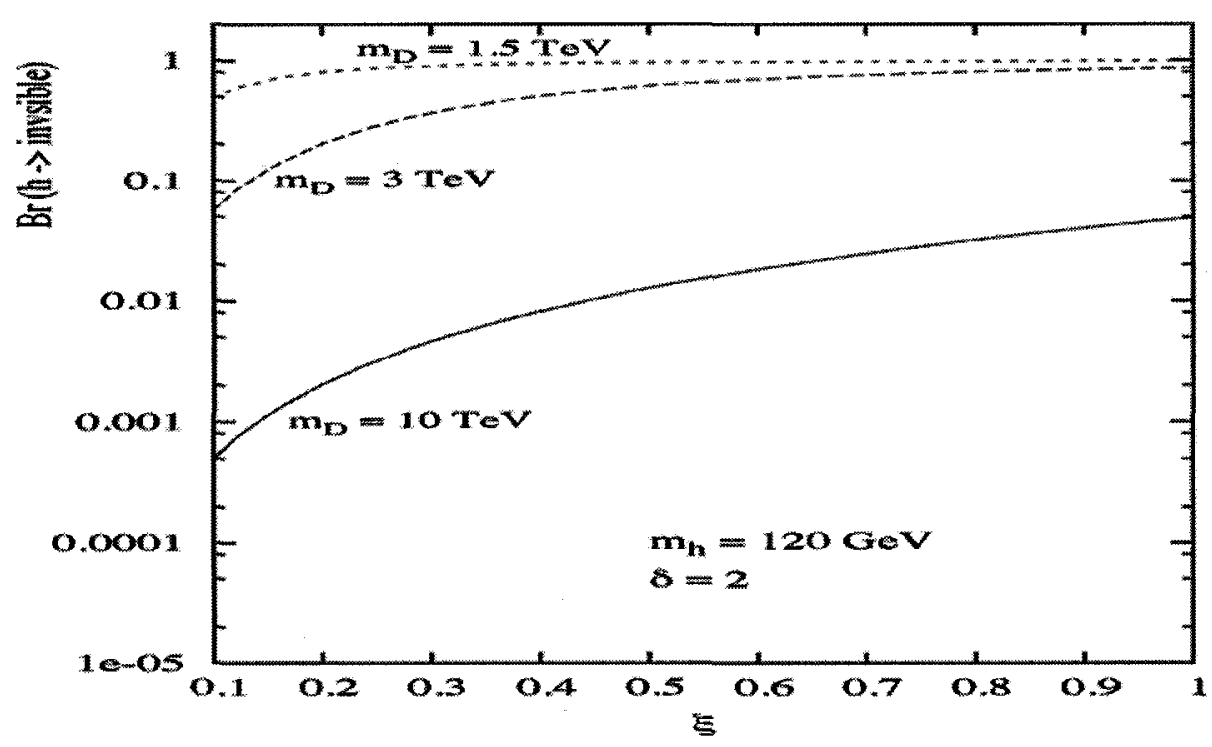

Figure 8.7: Invisible Higgs branching ratio, $\Gamma(h \rightarrow \mathcal{G})$, as a function of $\xi$ with two extra compactified dimensions for various $M_{D}$ energy scales [47] for a $120 \mathrm{GeV}$ Higgs mass.

$\xi^{2}$ as defined in Equation 8.7.

$$
\xi^{2}=B r(H \rightarrow i n v) \frac{\sigma_{B S M}}{\sigma_{S M}}
$$

Here, $\sigma_{B S M}$ represents the Beyond the Standard Model (BSM) production cross-section and $\sigma_{S M}$ is the SM cross-section. $\xi^{2}$ is usually expressed as a percentage of the SM production cross-section assuming $100 \%$ invisible decay. Therefore, a smaller value of $\xi^{2}$ indicates a higher sensitivity to the invisible Higgs process. A search for an invisible Higgs was performed by the LEP experiments consisting of data collected by ALEPH, DELPHI, L3, and OPAL. The LEP study investigated the Higgs-strahlung process, $e^{+} e-\rightarrow H Z$, for the $Z$ decay modes: $Z \rightarrow q \bar{q}$ and $Z \rightarrow \ell \ell$ where $\ell=e, \mu^{1}$. In the end, no evidence for an invisible Higgs was discovered and a lower bound for $m_{H}$ at $114.4 \mathrm{GeV}$ was set with $95 \%$ confidence level [48]. In addition, there have been several papers on searches at the Tevatron [36], however, the resolution on the missing transverse energy provides poor sensitivity to an analysis of this channel. Presently, there are no new limits based on

\footnotetext{
${ }^{1}$ The analysis from the DELPHI collaboration also included $Z \rightarrow \tau \tau$
} 
Tevatron data.

In addition to the studies using data, several analyses on the sensitivity of the ATLAS experiment for an invisible Higgs have been conducted. The following is a summary of these searches in order of production cross-section. These analyses were performed using a fast simulation ${ }^{2}$, however, in the case of the invisible Higgs a full simulation is preferred since detector effects such as cracks and mis-calibration can contribute to the tails of the missing transverse energy distribution. To this end, several new analyses are being performed using a fully simulated model of the ATLAS detector; one of these, a Higgs production via Vector Boson Fusion, is the subject of this thesis.

Gluon Fusion The gluon fusion production channel is the largest Higgs production channel at the LHC. The irreducible background for this channel is $Z \rightarrow \nu \nu$ with a crosssection $\sim 10^{4}$ times larger than the signal. Unfortunately, this channel provides no sensitivity because there is no means to separate the signal from the background.

Vector-Boson-Fusion Previous studies indicate that this process is the most promising production channel. It is characterized by two well separated jets and large missing energy. In addition, the color flow of the process leads to little activity between these two jets compared to QCD background processes. The irreducible background for this channel is the $Z+$ jet process, where $Z \rightarrow \nu \nu$. In addition, $W+$ jets and QCD dijets backgrounds were considered. This fast simulation analysis indicated a sensitivity to a Higgs decaying invisibly of $\xi^{2} \sim 25 \%$ at the $95 \%$ confidence level for a $130 \mathrm{GeV}$ Higgs mass using 30 $\mathrm{fb}^{-1}$ of data, as shown in Figure 8.8. One of the key concerns with the VBF channel is the ability to trigger on signal events within the required trigger rate. This concern is also investigated in this thesis.

\footnotetext{
${ }^{2}$ A description of the fast detector simulation is presented in Section 9.4.2.
} 
WH Associated Production A study for this production channel was performed in the case where $W \rightarrow \ell \nu$, and $\ell=e, \mu$ [49]. This channel has been shown not to be possible because of the overwhelming $\mathrm{W}$ inclusive background. This is a consequence of the inability to suppress the background events which populate the tail of the $\mathrm{W}$ transverse mass distribution.

ZH Associated Production Several studies have been carried out for this production channel requiring that the $Z$ boson decays into two leptons $[49,50]$. The main backgrounds considered in these analyses are the $\mathrm{ZZ}, \mathrm{ZW}, \mathrm{WW}$, and $t \bar{t}$. The $\mathrm{ZZ}$ background is the most challenging background since it has the same signal when one $Z$ decays leptonically and the accompanying $\mathrm{Z}$ decays into two neutrinos. The second largest background is the $\mathrm{ZW}$ process, where the $\mathrm{Z}$ and $\mathrm{W}$ decay leptonically, because of the limited lepton identification range. Finally, the WW and $t \bar{t}$ background also contribute to the background when they decay leptonically. This production channel is currently being investigated using a fully simulated model of the ATLAS detector with a preliminary sensitivity of $\xi^{2} \sim 75 \%$ at the $95 \%$ confidence level for a $130 \mathrm{GeV}$ Higgs mass using $30 \mathrm{fb}^{-1}$ of data. This result is comparable to previous published results [50].

$t \bar{t} \mathrm{H}$ Associated Production The $t \bar{t} \mathrm{H}$ associated production mode has also been studied by the ATLAS collaboration $[50,51]$. The irreducible background for this channel is the $t \bar{t} Z$ productions where $Z \rightarrow \nu \nu$. In addition, the $t \bar{t}, t \bar{t} \mathrm{~W}, b \bar{b} \mathrm{Z}, b \bar{b} \mathrm{Z}$ backgrounds were considered. The result of this analysis, using a fast simulation, provides a sensitivity for an invisible Higgs of $\xi^{2} \sim 80 \%$ at the $95 \%$ confidence level for a $130 \mathrm{GeV}$ Higgs mass using 30 $\mathrm{fb}^{-1}$ of data. The results from this production mode are comparable to the $Z H$ production mode, as shown in Figure 8.8.

The sensitivity to observe an invisible Higgs by the ATLAS experiment using fast simulation is shown in Figure 8.8 for three key production channels, VBF, ZH, and $t \bar{t} \mathrm{H}$. From 
this plot it can be seen that the VBF production channel provides the best sensitivity for observing an invisible Higgs at the ATLAS experiment. It should be noted that these are

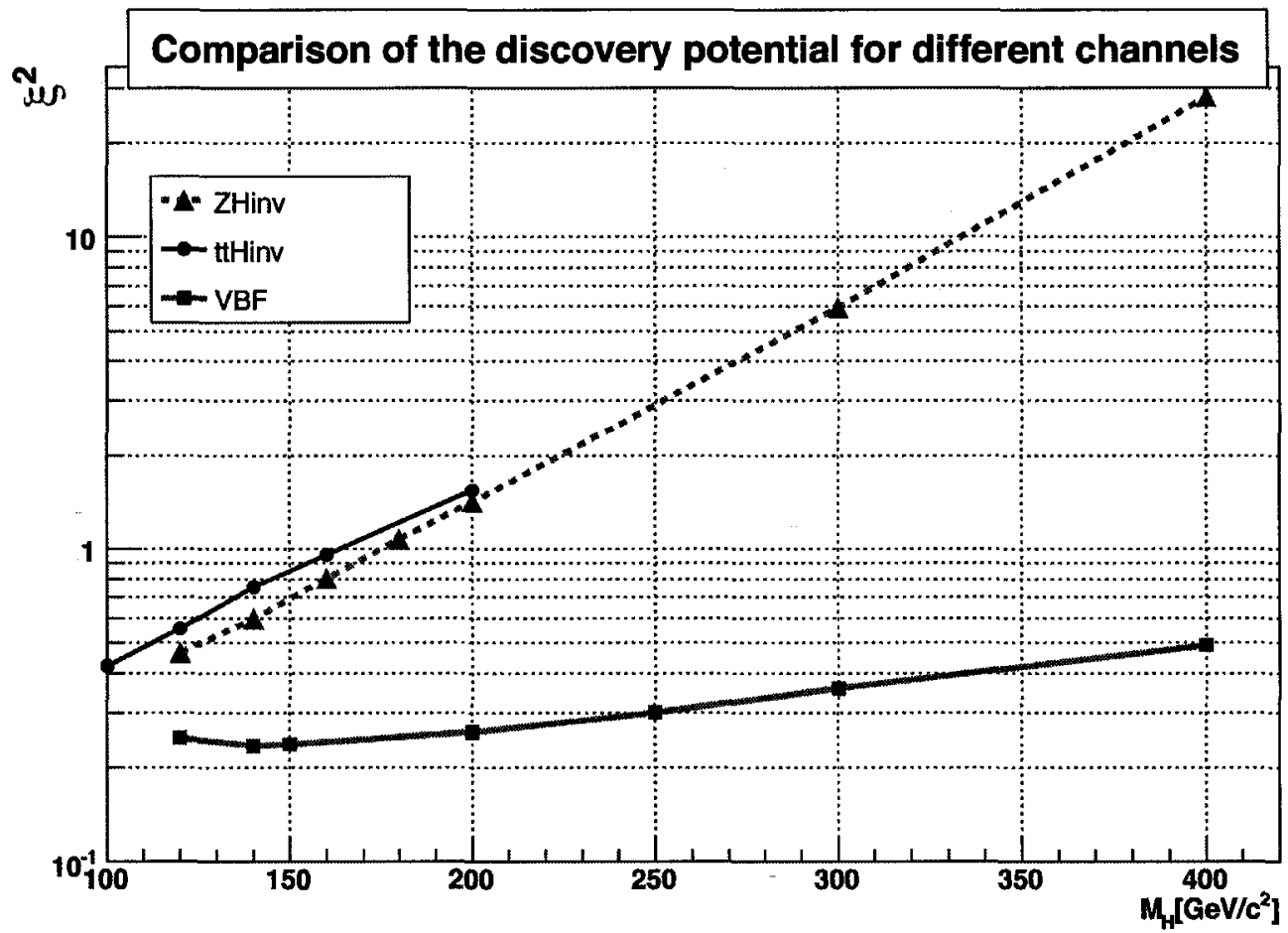

Figure 8.8: The $95 \%$ confidence level sensitivity limit, $\xi^{2}$, as a function of Higgs mass for invisible Higgs boson decays in the $\mathrm{ZH}, t \bar{t} \mathrm{H}$ and $\mathrm{VBF}$ production assuming an integrated luminosity of $30 \mathrm{fb}^{-1}$ using the ATLAS detector [50]. These analyses used fast simulation data.

generic analyses and the signal significance is examined with respect to SM backgrounds. Therefore, a measured excess in real data will indicate physics BSM. In the following chapters a summary of the procedure used to determine the sensitivity of an invisible Higgs via the VBF production channel using the fully simulated model of the ATLAS detector is presented. 


\section{Chapter 9}

\section{Monte-Carlo Event Generators and Data Production}

\subsection{Introduction}

This chapter reviews the ATLAS Monte-Carlo (MC) data production chain, specifically the production of the samples used for this thesis. The data production chain is separated into four stages, they are, in order of application: event generation, detector simulation, digitization and reconstruction, as shown in Figure 9.1. The event generator stage provides a set of particle 4-vectors from the hard scattering process of interest. Within the event generator, there can be several stages depending on the sample produced. These additional stages reflect the growing need for specialized Monte-Carlo event generator tools and will be discussed in Section 9.2. In addition, during event generation, filtering tools are sometimes used to remove events which are certain to fail the final analysis cuts. In Section 9.3.1, a summary of the samples generated for the analysis performed in this thesis are presented. Once the events are generated, they are passed to the detector simulation software. Section 9.4 summarizes two approaches used to simulate the detector depending on the level of detail required by the analysis and the available computing resources. Finally the output from the simulation stage is passed to the reconstruction 


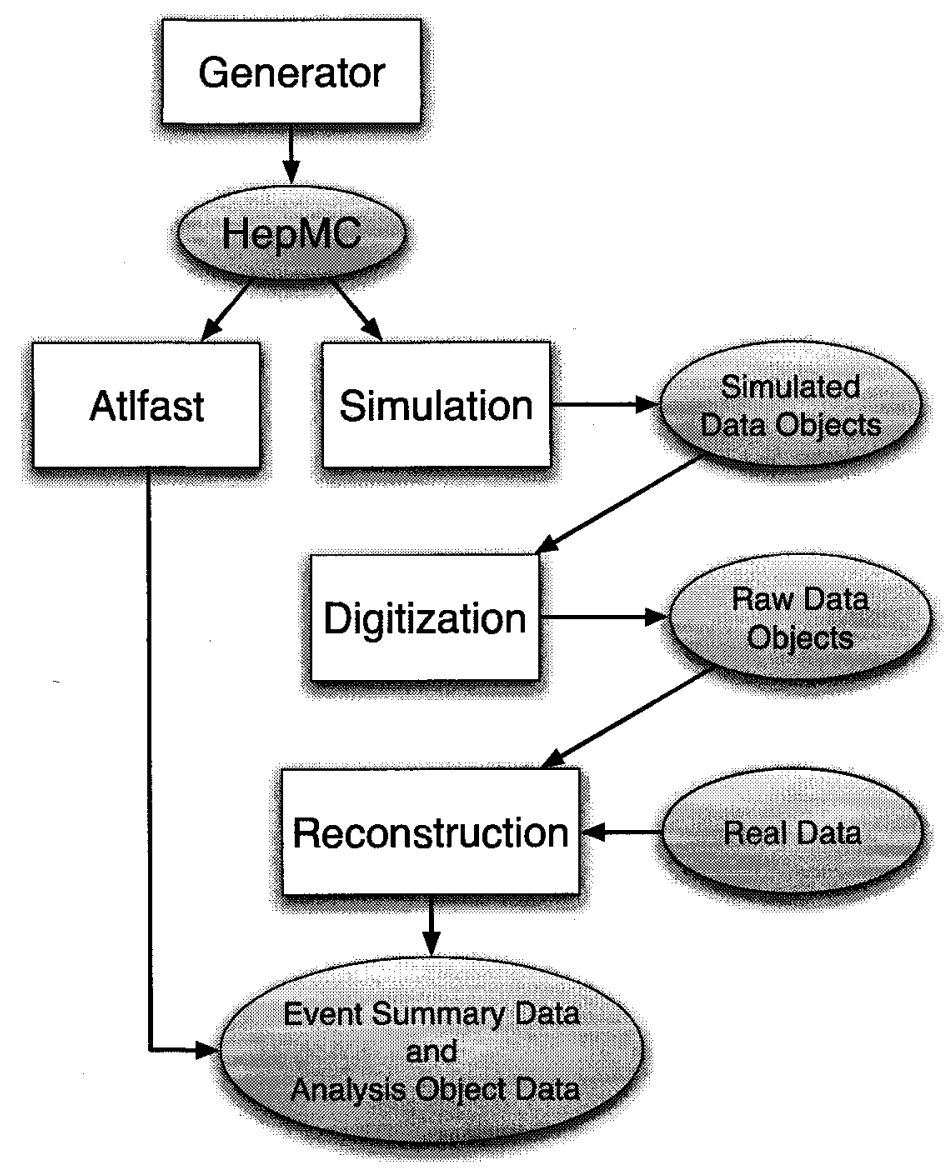

Figure 9.1: Schematic diagram of the ATLAS production sequence. The rectangular boxes represent the production stages and the oval boxes represent the output data. 
algorithms. The primary reconstruction algorithm used by the ATLAS collaboration will be reviewed in Section 9.5 .

By analyzing the simulated Monte-Carlo data, the ATLAS collaboration can develop and test all the reconstruction tools before real data is taken. The reconstruction algorithms include lepton identification and reconstruction, jet reconstruction, and the calculation of global event variables such as missing transverse energy, $\mathbb{E}_{T}$. There are two data output formats currently being used, the Event Summary Data (ESD) and the Analysis Object Data (AOD). The AOD format contains reconstructed information after detector calibration, such as jet 4 -vectors and $\mathbb{E}_{T}$. The information available in an AOD file continues to evolve based on the needs of the physics analyses. In contrast, ESD retains a more detailed description of the data which can be used to re-calibrate various data objects, such as jets, electrons, and muons.

\subsection{Event Generators}

Event generators are invaluable tools which bridge the gap between theoretical predictions and experimental data. This is done by decomposing the scattering process, in the case of the LHC proton-proton collisions, into four stages, the primary hard process, the Underlying Event (UE), massive particle decay, and hadronization. The primary hard process is the scattering process of interest. Everything in the collision, except this process of interest, is defined as the underlying event. The UE includes processes such as initial state radiation, final state radiation and additional parton-parton interactions. The initial (final) state radiation for proton-proton collisions involves gluons or quarks radiating off the colliding partons before (after) the primary hard process. Massive particles, such as the $\mathrm{Z}$ boson, created in the proton-proton collisions will subsequently decay. The final stage, called hadronization, involves the recombination of quarks and anti-quarks to create mesons and baryons. The validation of this decomposition approach is justified by 
the success in predicting a large amount of existing data using event generators such as Pythia and Herwig.

The complexity of the final state events at the LHC will be striking because of the high center-of-mass energy and this level of complexity requires a better understanding of each stage of the decomposition. Therefore, several new event generator tools have been developed that specialize in one of the stages of the scattering process. Such tools include Alpgen and Sherpa, which focus on the primary hard process by calculating the multiparton matrix elements, such as $\mathrm{W} / \mathrm{Z}+\mathrm{jets}, t \bar{t}+\mathrm{jets}$, and Jimmy which focusses on underlying events. The following is a brief summary of the event generators used to produce data for the analysis of this thesis.

\subsubsection{Herwig/Jimmy}

Herwig $[52,53]$ is a Monte-Carlo event generator that produces high-energy processes from lepton-lepton, lepton-hadron, and hadron-hadron scattering. Herwig provides a large number of different physics processes, including vector-boson-fusion from protonproton collisions. Herwig provides a complete package with all stages of event generation and also includes a specialized external program, such as Jimmy $[54,55]$ which provides a more accurate simulation of multiple parton scattering (underlying events) in hadronhadron collisions. It is for this reason that this generator was used to produce the signal events.

\subsubsection{Pythia}

Similar to Herwig, Pythia [56] is a Monte-Carlo event generator with an extensive list of available physics processes. It generates high-energy events from collisions between elementary particles, specifically $p p, p \bar{p}, e^{+} e^{-}$, and $e p$. Pythia also provides a complete package with all of the event generator stages, specifically the hard interaction process, initial and final state parton showers, underlying events, particle decays, and fragmenta- 
tion.

\subsubsection{Alpgen}

The Alpgen $[57,58]$ event generator is a specialized tool which focusses on the first step in the event generator process, the primary hard process. This tool provides leading order Matrix Element (ME) calculations for multi-parton final state events such as $W / Z+j e t s$, $t \bar{t}+$ jets. Alpgen provides the matrix element calculation for a specified number of final state partons. It can be interfaced with various event generators, such as Pythia and Herwig, which provide the additional event generator stages. To provide inclusive samples with no double counting of events, Alpgen applies a jet-parton matching scheme usually referred to as the MLM prescription [59]. In this thesis, the jet-parton matching efficiency is referred to as the MLM efficiency. The MLM matching scheme consists of the following four steps:

1. Define the hard process using Alpgen. This provides a set of partons from the ME calculations.

2. Perform parton showering (before hadronization) using Herwig/Jimmy or Pythia.

3. Construct jets using all the partons after parton showering. This includes the $\mathrm{ME}$ partons and the partons created during the showering in step 2.

4. Match the reconstructed jets to the ME partons within a defined matching cone size, $\Delta R=\sqrt{\Delta \eta^{2}+\Delta \phi^{2}}$, and a $p_{T}$ greater than a minimum $p_{T}$. For the samples produced for the analysis within this thesis, the matching cone size was set to 0.7 and the minimum $p_{T}$ required was $20 \mathrm{GeV}$.

Figure 9.2 provides examples to illustrate the matching process. An example of an exclusive sample where all jets are matched to a ME parton and the $\Delta R$ and minimum $p_{T}$ requirements are met is presented in Figure 9.2(a). Figure 9.2(b) provides an example of an inclusive sample where there are more jets than $\mathrm{ME}$ partons but all requirements are 
met. In contrast, Figures 9.2(c) and 9.2(d) provide examples of when the MLM requirement is not met. Figure 9.2(c) illustrates the case when two ME partons are collinear but fail the $\Delta R$ requirement and Figure 9.2(d) illustrates the case when the $p_{T}$ requirement is not met because one of the ME partons is too soft. The estimated systematic error associated with the MLM prescription is $\sim 10-20 \%$. In this thesis, Alpgen was interfaced with Herwig/Jimmy to provide the showering mechanism.

\subsection{Signal and Background Event Production}

In this section, the Monte-Carlo event generators used to produce the signal and backgrounds are reviewed. Events were generated within the context of the ATLAS Computer System Commissioning (CSC) data challenge process. This was designed to provide a consistent and validated set of MC data and to provide a means to test reconstruction algorithms.

\subsubsection{Invisible Higgs Signal}

The invisible Higgs signal, for which the Feynman diagram is shown in Figure 9.3(a), was generated using Herwig and Pythia. Because the Higgs mass is still unknown, multiple samples were produced in order to scan the mass range between 110-250 GeV. Herwig was used as the primary event generator for the signal instead of Pythia because of the variability of the central jet activity observed with different versions of Pythia. The signal was produced by generating a Higgs via the vector-boson-fusion mode and having the Higgs decay into two $\mathrm{Z}$ bosons. In turn, both $\mathrm{Z}$ bosons were forced to decay into neutrinos. The cross-section for the Higgs process is presented in Table 9.1 as a function of Higgs mass. 


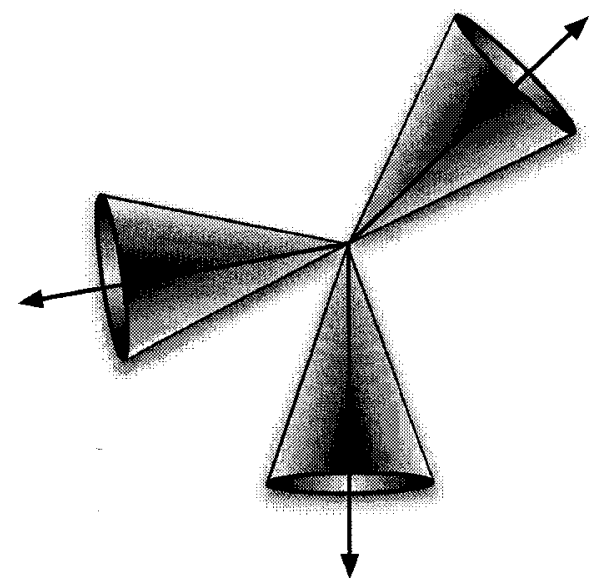

(a) Example of exclusive match

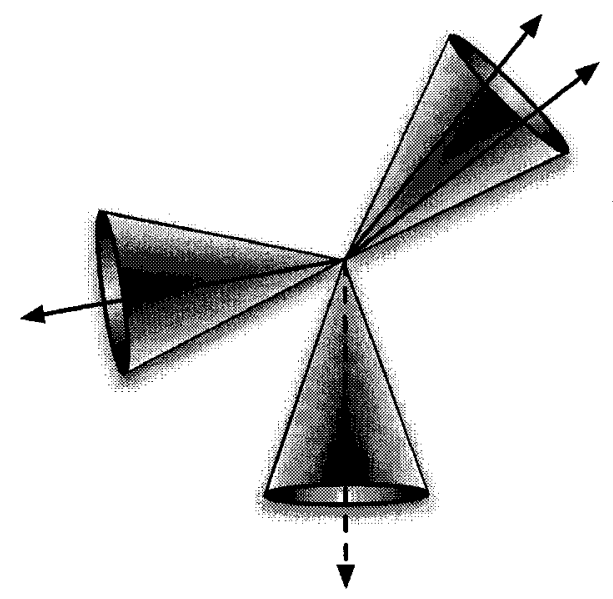

(c) Example of collinear partons

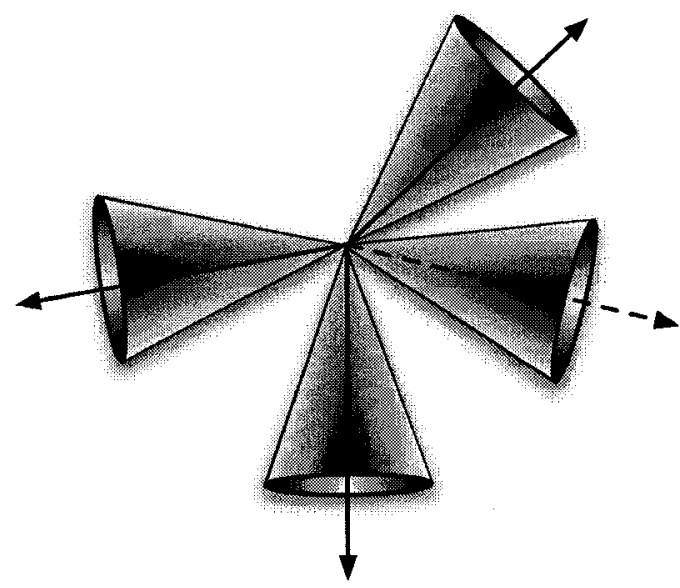

(b) Example of inclusive match

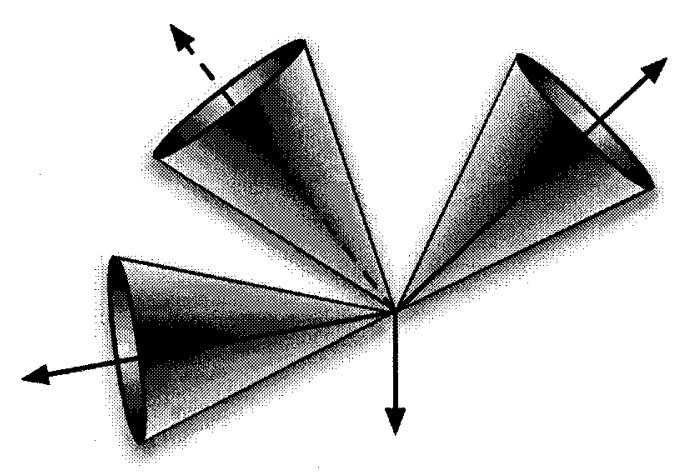

(d) Soft parton example

Figure 9.2: Illustration of the MLM prescription. The solid line represents the partons from the matrix elements calculation, the dashed line represents the partons produced during the showering stage, and the cones represent the jets constructed using all the partons after showering. The length of the parton lines is used as an indication of their $p_{T}$, as can be seen in Figure 9.2(d). 


\begin{tabular}{|c|c|}
\hline Higgs Mass [GeV] & Cross-section [pb] \\
\hline \hline 110 & 4.63 \\
130 & 3.93 \\
150 & 3.40 \\
200 & 2.41 \\
250 & 1.78 \\
\hline
\end{tabular}

Table 9.1: Leading-Order cross-sections for the vector-boson-fusion Higgs production mass scan analysis [60].

\subsubsection{The QCD Dijets Background}

The QCD dijet background events were considered because they have a very large crosssection and it is possible that detector deficiencies will allow them to mimic the signal. The large QCD dijet cross-section also has a significant impact on the trigger options studied for the VBF invisible Higgs channel. The unique topology of the signal provides a way to reduce the rate of this background with a suitable choice of trigger menus. Detector effects such as cracks and dead material are very important for this QCD dijet background as they can lead to a large apparent missing energy which resembles one of the characteristics of the signal. A study of these detector effects requires a full simulation analysis to properly understand the potential for this background to be misidentified as a signal.

Production of these samples requires large amounts of computing resources and the samples were produced on the ATLAS grid using Pythia 6.4 with ATLAS UE tuning. The ATLAS UE tuning is based on two hadron-hadron data sets with different colliding energies: $630 \mathrm{GeV}$ (UA5) and $1800 \mathrm{GeV}$ (Tevatron) [61]. Extrapolating from these data sets to the LHC energies provides the best model for the UE at LHC energies. The samples were divided into $p_{T}$ ranges to insure sufficient statistics throughout the complete $p_{T}$ spectrum. The cross-section and $p_{T}$ range for each sample is presented in Table 9.2. Approximately $100 \mathrm{k}$ events were analyzed for each $p_{T}$ range. 


\begin{tabular}{|c|c|c|}
\hline CSC Sample Name & $\sigma(p b)$ & $p_{T}$ range [GeV] \\
\hline \hline $\mathrm{J} 0$ & $1.76 \mathrm{E}+10$ & $8-17$ \\
\hline $\mathrm{J} 1$ & $1.38 \mathrm{E}+9$ & $17-35$ \\
\hline $\mathrm{J} 2$ & $9.33 \mathrm{E}+7$ & $35-70$ \\
\hline $\mathrm{J} 3$ & $5.88 \mathrm{E}+6$ & $70-140$ \\
\hline $\mathrm{J} 4$ & $3.08 \mathrm{E}+5$ & $140-280$ \\
\hline $\mathrm{J} 5$ & $1.25 \mathrm{E}+4$ & $280-560$ \\
\hline $\mathrm{J} 6$ & 360 & $560-1120$ \\
\hline $\mathrm{J} 7$ & 5.71 & $1120-2240$ \\
\hline
\end{tabular}

Table 9.2: Cross-section and $p_{T}$ range for the QCD dijet sample.

\subsubsection{The $Z+$ jets Background}

The $Z+$ jets background was divided into two decay modes, $Z \rightarrow \nu \nu$ and $Z \rightarrow \ell \ell$. The $Z \rightarrow \nu \nu$ is the most challenging background because it can resemble the signal by producing two jets and $\mathscr{E}_{T}$ with the same color flow as signal events, as shown in Figure 9.3(c). Color flow is associated with the quark and gluon lines in the hard scattering process. In the case of vector-boson-fusion, as shown in Figure 9.3(a), the quarks of the hard scattering process do not exchange color. In contrast, Figure 9.3(d) is an example of a process for which the quarks of the hard scattering process do exchange color. As a consequence, background events which have color flow can be suppressed by looking for quark or gluon activity between the primary scattered partons. The $Z \rightarrow \ell \ell$ samples were also generated as events in which leptons escape detection due to limited lepton $\eta$ coverage.

In a previous study of the invisible Higgs via VBF [62], Pythia version 6.158 was used to generate both the $\mathrm{W}+\mathrm{jets}$ and $\mathrm{Z}+\mathrm{jets}$ background. However, Pythia's implementation for these backgrounds only includes the matrix element terms for the $q q \rightarrow q V$ process, as shown in Figure 9.3(b), where $V$ represents either the $\mathrm{W}$ or $\mathrm{Z}$ vector boson. In other words, Pythia produces a $V+1$ jet and acquires the additional jets during the showering stage. It has been shown that Pythia's implementation underestimates the $Z+2$ jets process by $\sim 20-30 \%$ because it does not include the complete matrix element calculations $[62,63]$. A more significant problem with Pythia's implementation is that it does not include the 


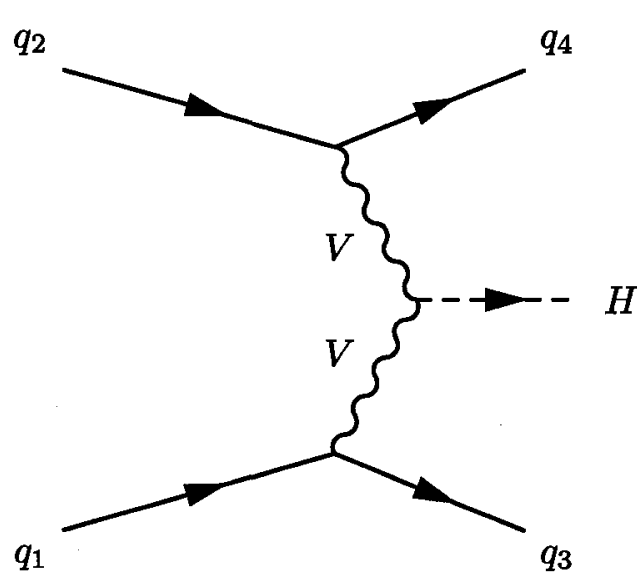

(a) Feynman diagram of Higgs production via (b) the vector-boson-fusion production mode.

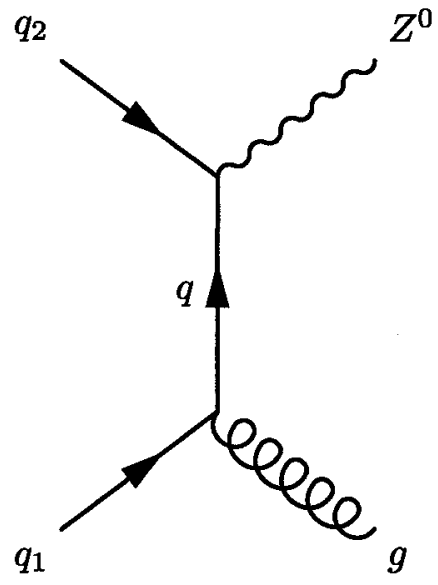

(b) Example of the hard scattering process for $\mathrm{Z}$ plus one parton.

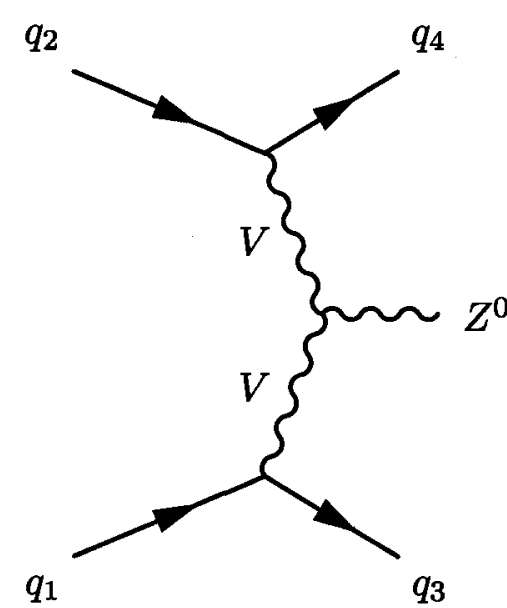

(c) Example of the hard scattering process for $\mathrm{Z}$ plus two partons via the electroweak process.

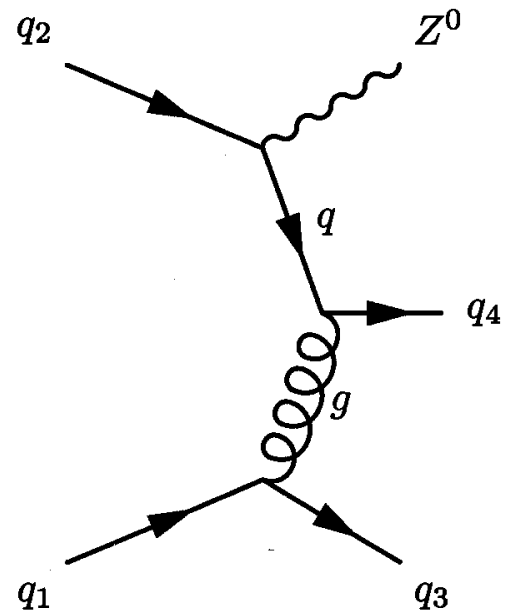

(d) Example of the hard scattering process for $\mathrm{Z}$ plus two partons via the QCD process.

Figure 9.3: Examples of the hard scattering processes. 


\begin{tabular}{|c|c|c|c|}
\hline Event Cut Flow & QCD+EW Efficiency & QCD Efficiency & Efficiency Ratio[\%] \\
\hline \hline $\mathbb{E}_{T}>80 \mathrm{GeV}$ & $2.81 \times 10^{-1}$ & $2.92 \times 10^{-1}$ & 96.2 \\
\hline Jet Tagging & $6.46 \times 10^{-3}$ & $5.08 \times 10^{-3}$ & 127 \\
\hline$M_{j j}>1200 \mathrm{GeV}$ & $1.44 \times 10^{-3}$ & $1.13 \times 10^{-3}$ & 127 \\
\hline$\not_{T}>100 \mathrm{GeV}$ & $9.69 \times 10^{-4}$ & $8.11 \times 10^{-4}$ & 120 \\
\hline Central Jet Veto & $7.54 \times 10^{-4}$ & $6.48 \times 10^{-4}$ & 116 \\
\hline $\mathbb{E}_{T}$ Isolation $>1 \mathrm{rad}$ & $3.63 \times 10^{-4}$ & $2.70 \times 10^{-4}$ & 134 \\
\hline$\Delta \phi_{j j}<1 \mathrm{rad}$ & $6.73 \times 10^{-5}$ & $5.40 \times 10^{-5}$ & 125 \\
\hline
\end{tabular}

Table 9.3: Particle level efficiency comparison between QCD and QCD+EW, where the efficiency ratio is defined as (QCD+EW)/QCD. See Section 10.2 for more detail on cut flow.

irreducible electroweak contribution, shown in Figure 9.3(c). Given these limitations, Alpgen was chosen to create this background. Within Alpgen, there are two different $Z+$ jets implementations, one which only includes QCD matrix element terms and the second which includes QCD and EW matrix element terms. In the second case bosons are created on shell without the $\mathrm{Z} / \gamma^{*}$ interference. In contrast, the first case includes these effects. However, it can be seen in Table 9.3 that using the process with only the QCD term for this analysis underestimates the background by $\sim 25 \%$. Therefore, all Alpgen samples used in this thesis include both QCD+EW terms. Three samples were produced using Alpgen for each of the two decay modes, $Z \rightarrow \nu \nu$ and $Z \rightarrow \ell \ell$. For each decay mode, two exclusive samples were produced containing either one or two final state partons and one inclusive sample was produced for three or more partons. Default Alpgen settings were used to generate events with the exception of a cut to remove very low $\mathbb{F}_{T}$ events by setting $\mathbb{E}_{T}>10 \mathrm{GeV}$ and an increase in the $\eta$ acceptance to $\left|\eta_{j}\right|<6$ and $\left|\eta_{\ell}\right|<6$ for both jets and leptons.

In addition to the Alpgen cuts, a filter was applied to the generated events to remove events which did not resemble the signal and will not survive the final offline cuts. This filter is composed of several kinematic cuts, as listed in Table 9.4, and is generally referred to as the vector boson filter. A study by J. Tanaka and S. Asai [64] was performed to investigate the bias introduced by this cut and is summarized in Table 9.5. It should be noted 


\begin{tabular}{|c|c|}
\hline Cut & Value \\
\hline \hline$p_{T}$ of highest $p_{T}$ tagged jet & $>20 \mathrm{GeV}$ \\
\hline$p_{T}$ of second highest $p_{T}$ tagged jet & $>15 \mathrm{GeV}$ \\
\hline$M_{j j}$, invariant mass of tagged jets & $>300 \mathrm{GeV}$ \\
\hline$|\delta \eta|$, absolute $\eta$ difference between tagged jets & $>2$ \\
\hline
\end{tabular}

Table 9.4: Cuts applied at the generator level.

\begin{tabular}{|c|c|}
\hline Number of Partons & Bias [\%] \\
\hline \hline 1 & $<0.49 \%$ \\
\hline 2 & $<0.11 \%$ \\
\hline 3 & $<0.03 \%$ \\
\hline
\end{tabular}

Table 9.5: Results of the study by J. Tanaka and S. Asai on VBF Cuts bias [64].

that the VBF cuts in their study have a set of cuts on the leptons, in addition to those presented in Table 9.4. Moreover, the offline cuts used in their analysis were looser than those used in this analysis. Most notably for the invariant mass of the tagged jets, $M_{j j}$, where their offline cut was $M_{j j}<700 \mathrm{GeV}$ in comparison to $M_{j j}<1200 \mathrm{GeV}$ in this thesis. Therefore, the biases presented in Table 9.5 are considered conservative assumptions for this analysis. A summary of the Alpgen event generator cross-section ( $\sigma$ ), MLM efficiency, vector boson filter efficiency, and effective cross-section $\left(\sigma^{\prime}\right)$ after both the MLM and the vector boson filter were applied, and MC events that survived MLM matching and filtering are presented in Table 9.6 for the $Z \rightarrow \nu \nu$ and $Z \rightarrow \ell \ell$ processes.

\subsubsection{The $\mathrm{W}+$ jets Background}

The $\mathrm{W}+\mathrm{jets}$ background was considered for the case in which the $\mathrm{W}$ decayed leptonically. The $F_{T}$ arises from the neutrino and in the case where the lepton escapes detection. The $\mathrm{W}+\mathrm{jets}$ background was generated in the same manner as the $\mathrm{Z}+\mathrm{jets}$ background. $\mathrm{A}$ summary of the event generator cross-section $(\sigma)$, MLM efficiency, vector boson filter efficiency, and effective cross-section $\left(\sigma^{\prime}\right)$ after both the MLM and the vector boson filter were 


\begin{tabular}{|c|c|c|c|c|c|c|}
\hline & Parton \# & $\sigma(p b)$ & MLM Eff. & VBF Filter Eff. & $\sigma^{\prime}(p b)$ & MC events \\
\hline \hline$Z \rightarrow \nu \nu$ & 1 & 2975.88 & 0.5395 & 0.0411 & 65.99 & $25 \mathrm{k}$ \\
\hline & 2 & 1293.49 & 0.3983 & 0.1155 & 59.51 & $65 \mathrm{k}$ \\
\hline & 3 (inclusive) & 499.75 & 0.4194 & 0.3574 & 74.91 & $32 \mathrm{k}$ \\
\hline \hline$Z \rightarrow \ell \ell$ & & & & & & \\
\hline & 1 & 1507.45 & 0.5395 & 0.0434 & 35.30 & $25 \mathrm{k}$ \\
\hline & 3 & 655.65 & 0.3972 & 0.1210 & 31.51 & $24 \mathrm{k}$ \\
\hline \hline & & & & 0.3664 & 38.66 & $23 \mathrm{k}$ \\
\hline \hline$W \rightarrow \ell \nu$ & 1 & 15135.70 & 0.5509 & 0.0422 & 351.87 & $25 \mathrm{k}$ \\
\hline & 2 & 6480.12 & 0.4047 & 0.1178 & 308.93 & $24 \mathrm{k}$ \\
\hline & 3 (inclusive) & 2407.67 & 0.4198 & 0.3583 & 362.15 & $24 \mathrm{k}$ \\
\hline \hline
\end{tabular}

Table 9.6: $\mathrm{W} / \mathrm{Z}+$ jet background samples divided by parton samples for the process using Alpgen/Jimmy. The "MC events" are the number of MC events that survive MLM matching and filtering.

applied are presented in Table 9.6.

\subsection{Detector Simulation}

There are two approaches within ATLAS that are used to simulate particle interactions within the detector and these are usually referred to as full and fast simulation. The full simulation provides a detailed representation of the detector and is composed of a simulation and a digitization stage. However, the detailed representation provided by the full simulation approach requires large amounts of data storage and processing time. In contrast, the fast simulation uses a parameterized approach that is 4 to 5 orders of magnitude faster and requires less data storage. The following sections will summarize both approaches.

\subsubsection{Full Simulation}

The full simulation approach uses generated events provided by the event generation stage and processes them using the complete Geant $4[65,66]$ model of the ATLAS detector. Geant4 provides a framework for the detector description, propagation of particles 
within the detector, material description, and the modeling of particle interactions with the material of the detector. Geant 4 allows the user to define the level of detail needed to describe the detector and particle interactions. In addition, Geant 4 provides the flexibility to define active and absorbing elements of the detector which is invaluable for studying calorimeter calibration. The ATLAS collaboration has implemented a very detailed detector description and as a consequence its use within Geant 4 requires a significant amount of computing resources. However, this level of detail provides crucial information such as the proper simulation of cracks within the detector which affect the missing transverse energy resolution. It is for this reason that all the data produced for the analysis in this thesis was generated using the full simulation approach.

Once the full simulation is complete, the information is passed to the digitization stage. The digitization stage re-processes the simulated data and applies detector effects such as front-end electronics noise and pile-up. The output from the digitization stage is intended to reflect the raw data produced by the actual experiment.

\subsubsection{Fast Simulation}

Fast simulation uses a parameterized approach to take detector effects into account and is 4 to 5 orders of magnitude faster and requires less storage space than the full simulation approach. Therefore, this approach is ideal for feasibility studies and analysis which require large data samples.

The application which performs this task in ATLAS is called AtlFast [67]. As an example of the fast simulation, calorimeter cell energies are determined by summing the transverse energy deposits of every particle ${ }^{1}$ using a default $\eta \times \phi$ granularity of $0.1 \times 0.1$ for $\eta<3$ and $0.2 \times 0.2$ for $\eta>3$. Afterwards, the cells are clustered using a cone algorithm and kept if the clusters have $p_{T}>10 \mathrm{GeV}$. The 4-vectors corresponding to electrons, pho-

\footnotetext{
${ }^{1}$ Except neutrinos and muons.
} 
tons and muons are smeared based on parameterized values derived from test-beam and full simulation results and the associated calorimeter clusters are identified and removed. From the remaining clusters, jets are reconstructed and kept if $p_{T}>15 \mathrm{GeV}$. Similar to the leptons, these reconstructed jets are smeared based on parameterized values. The $\mathbb{E}_{T}$ is calculated using the sum of all the clusters and smeared using the same jet parameterized values. Results from AtlFast are stored in AOD format.

\subsection{Atlas Reconstruction}

The ATLAS detector, as summarized in Chapter 2, is an extremely complex detector designed to identify various particles and to measure some of their properties, such as momentum, charge, and mass. In addition, the ATLAS detector must also be able to measure global quantities such as missing transverse energy. In this chapter, the algorithms used to identify and reconstruct properties of particles are briefly summarized.

\subsubsection{Electron Reconstruction}

Electrons are identified using the combined information of the inner detector and calorimeter system. Calorimeter information is used to find seed clusters using a sliding window algorithm. The window slides towers over an $\eta-\phi$ grid to find local energy peaks. Many corrections are applied to these clusters to account for biases. An electron candidate requires the seed cluster to be spatially matched to a good quality track.

In order to separate electrons from jets, electron candidates must pass a series of cuts based on the properties of the shower shape for different layers of the calorimeter as well as various combinations of inner detector and calorimeter information. A total of 13 requirements (bits) must be met in order to identify an electron ${ }^{2}$.

\footnotetext{
${ }^{2}$ The number of requirements needed to identify a candidate can be loosened by accessing the actual bit mask.
} 


\subsubsection{Muon Reconstruction}

Muon identification is achieved primarily through the use of the inner detector and the muon spectrometer. Unlike other charged particles, muons provide tracks in both the inner detector and muon spectrometer which can be combined to identify a muon. In principle, tracks are first reconstructed within each sub-system and then combined. However, the magnetic field and multiple scattering effects must be carefully considered in order to properly identify a muon.

There are several muon algorithms available within the ATLAS reconstruction software. There are two muon reconstruction chains used, they are: STACO and MUID. Each chain has four algorithms which can be divided into stages. These stages are: Muon Spectrometer (MS) tracking, Inner Detector (ID) tracking, combined tracking algorithms, and low $p_{T}$ combined tracking algorithms. For particles with $p_{T}<100 \mathrm{GeV}$, the inner detector provides better momentum resolution than the muon spectrometer, while at higher $p_{T}$ the muon spectrometer provides the best momentum determination.

\subsubsection{Hadronic Tau Reconstruction}

The $\tau$ leptons decay hadronically $\sim 65 \%$ of the time and the remainder decay into electrons and muons. Since electron and muon identification has been covered in Sections 9.5.1 and 9.5.2, this section covers $\tau$-jet identification and reconstruction.

In a hadronic $\tau$ decay, the $\tau$ will produce a single track $\sim 77 \%$ of the time:

$$
\tau^{ \pm} \rightarrow \nu_{\tau}+\pi^{ \pm}+n \pi^{0}
$$

Approximately all remaining hadronic $\tau$ decays produce three tracks:

$$
\tau^{ \pm} \rightarrow \nu_{\tau}+3 \pi^{ \pm}+n \pi^{0}
$$


Therefore, in order to identify a $\tau$-jet, a combination of inner detector and calorimeter information is used, requiring a calorimeter cluster and associated tracks.

There are two algorithms developed by ATLAS to reconstruct a $\tau$-jet, they are: TauRec and Tau1P3P.

TauRec is the official ATLAS $\tau$-jet reconstruction and identification algorithm within $|\eta|<$ 2.5. This algorithm scans for a valid seed, which is defined as a calorimeter cluster or jet with $p_{T}>15 \mathrm{GeV}$. Once a valid seed is found, TauRec requires at least one associated track within $\Delta R<0.3$ of the seed with a $p_{T}>2 \mathrm{GeV}$. This procedure defines a $\tau$-jet candidate. Detector variables, such as the jet radius in the electromagnetic calorimeter and the number of associated charged tracks, are used to discriminate $\tau$-jets from normal jets. Once a $\tau$-jet has been identified, it is calibrated using cell-based energy density information.

Tau1P3P is intended to reconstruct "soft" $\tau$ jets. It is driven by the difference in the detector performance of the inner tracking and the calorimeter systems, namely that the $p_{T}$ resolution of the tracking system is expected to be better at $p_{T}<110 \mathrm{GeV}$ than that of the calorimeter system, as illustrated in Figure 9.4. Tau1P3P relies on the hadronic $\tau$ topology to identify a $\tau$, i.e. one (1P) or three (3P) charged tracks. Tau1P3P requires a "good" quality track with $p_{T}>9 \mathrm{GeV}$ as a seed. Afterwards, Tau1P3P searches for additional "good" quality tracks with $\Delta R<0.2$ and a $p_{T}>2 \mathrm{GeV}$. Once a $\tau$ jet candidate is found, cuts similar to the one applied by TauRec, are used to reject normal jets. The $\tau$ jets are then calibrated using the $p_{T}$ of the track instead of the $E_{T}$ from the calorimeter. There are plans to merge both algorithms into one algorithm, preserving the best features from both. 


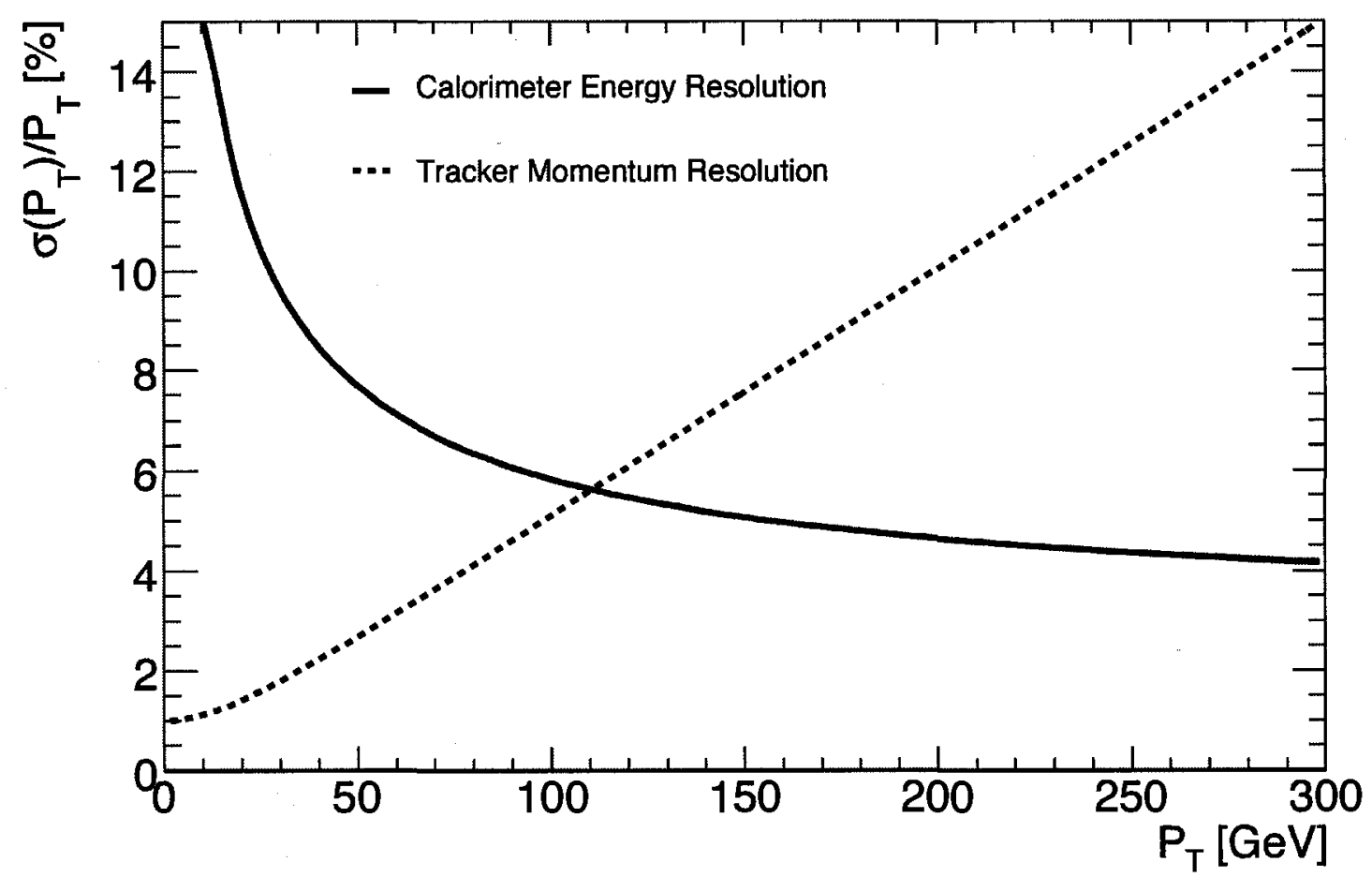

Figure 9.4: Comparison between calorimeter energy resolution (solid line) and tracker momentum resolution (dashed line) as function of $p_{T}$.

\subsubsection{Jet Reconstruction}

Jet algorithms provide a technique to map the observed hadronic final states to the kinematics of the partons. The jet algorithms used in ATLAS look for energy clusters in the calorimeter cell information. There are however, a large number of calorimeter cells in ATLAS, so one of two techniques are used for pre-clustering, they are: CaloTowers and CaloTopoClusters. A CaloTower is a tower of cells on a fixed $\Delta \eta \times \Delta \phi$ grid with a typical size of $0.1 \times 0.1$. Towers with negative noise are combined with neighboring towers until the net sum is positive. In contrast, a CaloTopoCluster is a collection of cells based on a three-dimensional topological cluster algorithm [68]. The topology is based on the cell's signal-to-noise ratio. A CaloTopoClusters is seeded by a cell with a minimum signal-tonoise ratio and neighboring cells are added if they meet a lesser signal-to-noise value.

Once the towers or topoclusters are reconstructed, the actual jet algorithm is applied. 
There are several jet algorithms available within the ATLAS reconstruction software. However, the Cone and $K_{T}$ algorithms [69] are the two default jet algorithms used by the ATLAS collaboration. The seeded cone algorithm has two setting parameters, they are: a transverse energy seed threshold and a cone size. The cone size represents an area of $A=\pi R^{2}$ in the $\Delta \eta \times \Delta \phi$ plane. Particles within this cone are combined for a jet. In contrast, the $K_{T}$ algorithm collects all particles by "merging" the particles in order of increasing $p_{T}$. The "merging" is controlled by a parameter which defines the effective size of the jet.

Up to now the jets are uncalibrated, that is, they are reconstructed using the electromagnetic scale calibration. However, the ATLAS calorimeters are under-compensating detectors, therefore additional calibrations must be applied. To this end, the first stage in calibrating jets involves applying H1-Style weights [70]. These weights are applied at the readout cell level and rely on the cells energy density, $\rho=E / V$. However, this calibration is not universal and does not correct for the jet location or jet algorithm effects. These mis-calibrations are corrected using $\eta$ and $p_{T}$ dependent functions for each jet. In addition to these calibration schemes, it is possible to extract further corrections using in-situ calibrations. These calibrations require final state events with a well measured electromagnetic object balancing one or more jets. It is expected that this additional calibration can provide a jet energy uniformity in $\eta$ of about $2 \%$.

\subsubsection{Missing Transverse Energy Reconstruction}

There are several searches which require a good measurement of the missing transverse energy, $\mathbb{E}_{T}$, such as the invisible Higgs searches, top quark mass reconstruction, and Higgs searches involving taus. The additional $\eta$ coverage provided by the FCal was designed to reduce the tails from particles going down the beam line. However, there are several transition regions which can also introduce tails in the $\mathbb{E}_{T}$ distribution and must be corrected. 
The missing energy is calculated by summing the energy deposited in the $\mathrm{x}$ and $\mathrm{y}$ direction in the calorimeter and the muon system. The $\mathbb{E}_{T}$ term in the muon system is determined using the stand-alone muon spectrometer reconstruction algorithm in order to prevent double counting of energy deposited in the calorimeter system. The calorimeter term is calculated using the calibrated calorimeter cell information. These cells are calibrated using the same H1-weights used by the reconstructed jet algorithms. In order to suppress the noise from the calorimeter cells, only cells with energy above a cut related to the noise threshold, $\left(\left|E_{\text {cell }}\right|>2 \sigma_{\text {cell }}\right)$, are used, where $\sigma_{\text {cell }}$ is the noise in the cell.

As with jets, the H1-weights do not correct for effects such as the dead material of the cryostat. Therefore, these effects are corrected using functions that depend on $\eta$ and $p_{T}$. It should be noted that the weights used for this function are not the same as for jets as the weights applied to jets also correct for additional effects that arise from the jet algorithm used. In addition, a refined calibration is applied to different reconstructed objects, such as electrons and jets.

The performance of the $\mathbb{E}_{T}$ algorithm before calibration (i.e. electromagnetic scale) was studied and shown to have a large systematic bias in the energy response of $\sim 10-20 \%$. However, once the calibration weights were applied to the calorimeter cells and the muon reconstruction included, the response was better than $5 \%$. In addition, the performance of the $\mathbb{E}_{T}$ azimuthal angle resolution was studied. A good $\mathbb{E}_{T}$ azimuthal angle resolution is required to remove background events, such as QCD dijets, with fake high $\mathbb{E}_{T}$. These fake $\mathscr{E}_{T}$ events can arise when a high $p_{T}$ jet is mis-measured and a large amount of their energy is deposited in un-instrumented regions of the ATLAS detector. A common way to remove these events is to apply a cut on the difference in the azimuthal angle between the jets and $\mathbb{E}_{T}$. For events with true $\mathbb{E}_{T}$ above $\sim 80 \mathrm{GeV}$ the azimuthal resolution of $\mathbb{E}_{T}$ is better than 0.2 radians. 


\subsubsection{Event Building}

The previous sections reviewed the algorithms used to reconstruct particles used for this analysis. For every event, all possible particle objects, such as electrons, muons, taujets, jets, and $\mathbb{E}_{T}$ are reconstructed. However, in any given event there might be several reconstructed particles which overlap in the same physical location, in other words one physical object has been assigned more than one identity. In this case, one must chose an identity for the object. Within the ATLAS software, several tools have been developed to address this problem, such as EventView [71]. The general idea of overlap removal within EventView is to provide a tool with which a user can define an order of precedence. For example, a user can define the order of precedence to be: electron, muon, tau-jets, and jets. In this case, the electron will always be chosen over any other defined object. In order to provide flexibility, the user can define multiple "views" in which the order of precedence, the reconstruction algorithm used, and various cuts and requirements can be changed. This provides a powerful tool for studying systematic errors and for testing new algorithms. 


\section{Chapter 10}

\section{Sensitivity Study for an Invisible}

\section{Higgs via the Vector Boson Fusion}

\section{Channel}

Presented in this chapter are two studies that investigate the sensitivity for detecting an invisible Higgs for Higgs masses between 110 to $250 \mathrm{GeV}$ using the ATLAS detector. These studies are based on fully reconstructed signal and background events. Section 10.1 reviews the methodology used to reconstruct an event, which includes defining the reconstruction algorithms and the procedure used to remove overlapping reconstructed leptons and jets. In Section 10.2, the selection cuts applied for both sensitivity analyses are presented. A feasibility study for the level 1 trigger is presented in Section 10.3. In Sections 10.4 and 10.5 , the sensitivity studies based on the selection cuts and the shape analysis are presented. Section 10.6 investigates the key systematic errors for this analysis. Concluding, the ATLAS sensitivity for an invisible Higgs at a $99 \%$ confidence level limit is presented. 


\subsection{Analysis Framework}

The analysis performed for the VBF invisible Higgs search was based on an adaptation of the ATLAS EventView package. This was used to assign identities to each reconstructed object which requires the definition of an order of precedence. In this analysis, the order of precedence used was muons, electrons, $\tau$-jets, and jets.

Muon Reconstruction Muon candidates were reconstructed using the "Staco" algorithm which statistically merges two independent measurements from the muon chambers and the inner detector [72]. At the EventView level, muons were selected with a $p_{T}>15 \mathrm{GeV}$. The identification efficiency based on the events in the Alpgen $\mathrm{Z}(\rightarrow \ell \ell)+\mathrm{jets}$ samples was found to be $91 \%$, when requiring the truth muons to be within $|\eta|<2.5$ and have a $p_{T}>$ $20 \mathrm{GeV}$.

Electron Reconstruction Electron candidates were determined using the "egamma" algorithm [73], which identifies and reconstructs electrons and photons based on their shower shape in the calorimeter and tracking system. At the EventView level, electrons were selected with a $p_{T}>15 \mathrm{GeV}$. The identification efficiency based on the events in the Alpgen $\mathrm{Z}(\rightarrow \ell \ell)+\mathrm{jets}$ samples was found to be $70 \%$, when requiring the truth electrons to be within $|\eta|<2.5$ and have a $p_{T}>20 \mathrm{GeV}$.

$\tau$-jet Reconstruction $\tau$-jet candidates were reconstructed using the calorimeter based algorithm, "tauRec" [74-76]. TauRec reconstructs $\tau$-jets using information from the calorimeter and the inner detector. The identification efficiency based on the events in the Alpgen $\mathrm{Z}(\rightarrow \ell \ell)+$ jets samples was found to be $60.2 \%$, when requiring the truth $\tau$-jets to be within $|\eta|<2.5$ and have a $p_{T}>30 \mathrm{GeV}$.

Jet Reconstruction The jet candidates were reconstructed using a topocluster-based cone algorithm with a cone size of $0.4[68,69]$. The identification efficiency based on the events 
for the Alpgen $\mathrm{Z}+\mathrm{jets}$ and $\mathrm{W}+\mathrm{jets}$ samples was found to be $93 \%$, when requiring the truth jets to be within $|\eta|<5$ and have a $p_{T}>40 \mathrm{GeV}$.

Missing Transverse Energy Reconstruction The missing transverse energy, $\mathbb{E}_{T}$, was reconstructed by summing the contributions from cells in electrons/gamma, muons, and jet objects. The jets were calibrated using an energy density weighting scheme referred to as H1-weights. In addition, contributions from calorimeter cells collected by a topological clustering algorithm which were not included in the jet collections and cryostat corrections, were included in the $\mathbb{E}_{T}$ calculation.

\subsection{Selection Cuts}

In this section, the selection cuts used for this analysis are presented. These cuts are based on those used in an earlier ATLAS study [62] and are motivated by the kinematic distributions of the signal and background.

Tagged Jets: In order for an event to be considered, a minimum of two jets $j_{1}$ and $j_{2}$ are required. These two jets are the highest $p_{T}$ jets of the event and are expected to represent the recoil jets from the VBF process. In this thesis, these two jets are referred to as "tagged" jets. To maximize the jet tagging efficiency the full acceptance of the detector was used, that is $\left|\eta_{j 1,2}\right|<5$. Once two tagged jets had been identified, they were each required to have a $p_{T}$ greater than $40 \mathrm{GeV}$. This $p_{T}$ cutoff removed events with a "soft" tagged jet that were primarily produced in QCD dijets samples. The $p_{T}$ distributions of the tagged jet for the signal and backgrounds are presented in Figure 10.1. In Figures 10.1(a) and 10.1(b) there is a large difference in the $p_{T}$ of the tagged jets between the signal and the QCD dijet background. In contrast, leading $p_{T}$ jets from $\mathrm{W} / \mathrm{Z}+\mathrm{jets}$ background events are very similar to those from signal events. This can be understood since the leading $p_{T}$ jets of the $\mathrm{W} / \mathrm{Z}+$ jets background have the same recoil as the signal. Cuts on the product and difference of the pseudorapidity of the two tagged jets were also used. They were 


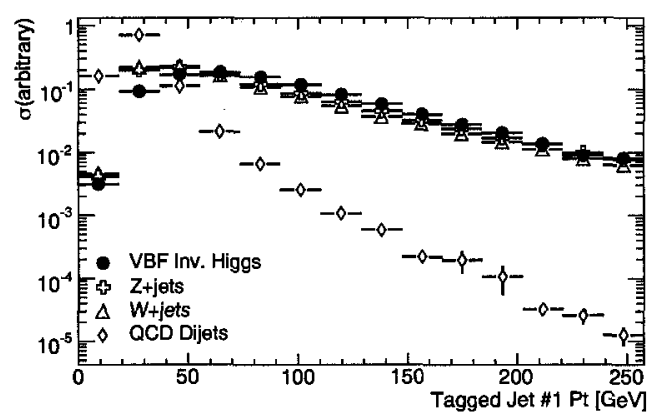

(a) $p_{T}$ of tagged jet with highest $p_{T}$.

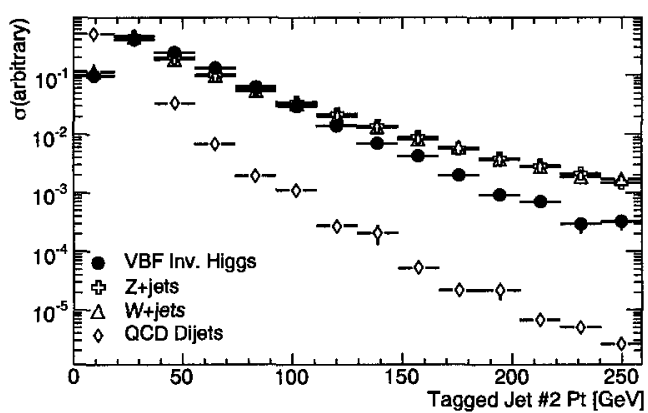

(b) $p_{T}$ of tagged jet with second highest $p_{T}$.

Figure 10.1: Cross-section in arbitrary units for the signal and background as a function of the $p_{T}$ of the tagged jets. Signal events are for a $130 \mathrm{GeV}$ mass shown in bullets and background samples $\mathrm{Z}+\mathrm{jets}, \mathrm{W}+\mathrm{jets}$, and QCD dijets are shown in open crosses, triangles, and diamonds, respectively. The distributions are normalized to unity.

$\eta_{j 1} \cdot \eta_{j 2}<0$ and $\Delta \eta>4.4$, respectively. Distribution of $\eta_{j 1} \cdot \eta_{j 2}$ and $\Delta \eta$ for the signal and background are presented in Figure 10.2. Figures 10.2(a) and 10.2(b), show the separation in the distribution of these variables for both the signal and background.

Invariant Mass of the Tagged Jets: The majority of the QCD dijet background will produce soft jets resulting in a low invariant mass from the two tagged jets, $M_{j j}$, as shown in Figure 10.3. Figure 10.3(a) shows $M_{j j}$ distributions without any offline cuts and clearly illustrates the discriminating power of this variable. However, it should be noted that for the $\mathrm{W} / \mathrm{Z}+$ jets background events, a cut of $M_{j j}>300 \mathrm{GeV}$ was applied during event generation as discussed in Section 9.3.3. The effect of this cut can be seen in Figure 10.3(a) which shows a flattening of the $M_{j j}$ distribution between 0 and $\sim 400 \mathrm{GeV}$ for the $\mathrm{W} / \mathrm{Z}+$ jets backgrounds. The offline invariant mass cut of $1200 \mathrm{GeV}$ was chosen to further reduce the $\mathrm{W} / \mathrm{Z}+$ jets background and was motivated by the distribution shown in Figure 10.3(b).

Missing Transverse Energy: The primary signature for the VBF invisible Higgs channel is a large missing transverse energy from the Higgs decaying invisibly. A cut on $E_{T}$ significantly reduces the QCD dijet and $\mathrm{Z}(\rightarrow \ell \ell)+$ jets backgrounds since no significant $\mathbb{E}_{T}$ is 


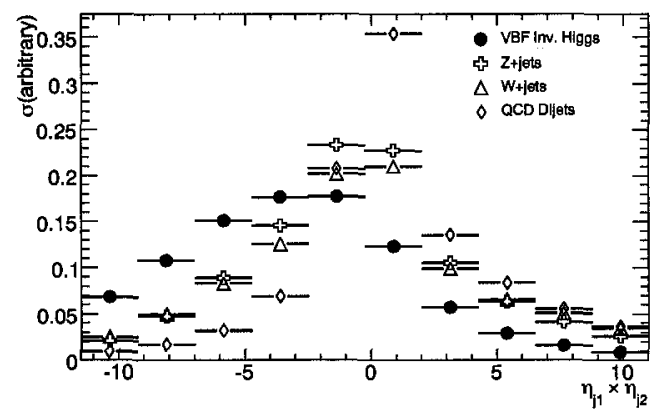

(a) Tagged jet $\eta$ product $\left(\eta_{j 1} \times \eta_{j 2}\right)$

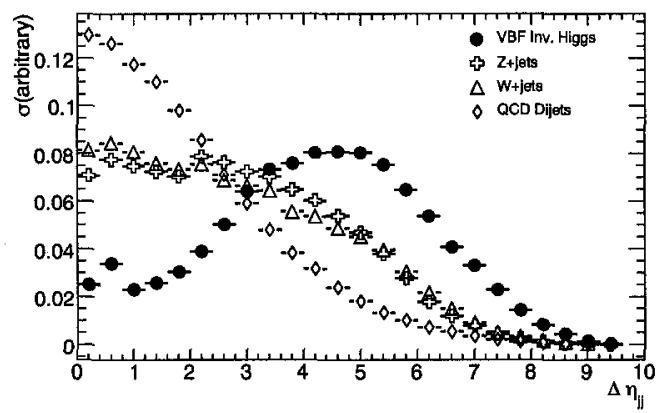

(b) Tagged jet $\Delta \eta=\left|\eta_{j 1}-\eta_{j 2}\right|$. The flattening of the distribution for the $\mathrm{W} / \mathrm{Z}+$ jet samples is a result of the VBF filtering cut discussed in Section 9.3.3.

Figure 10.2: Cross-section in arbitrary units for the signal and background as a function of tagged jet $\eta$ dependent variables with no cut on the $p_{T}$. Signal events are for a 130 $\mathrm{GeV}$ mass shown in bullets and background samples Z+jets, W+jets, and QCD dijets are shown in open crosses, triangles, and diamonds, respectively. The distributions are normalized to unity.

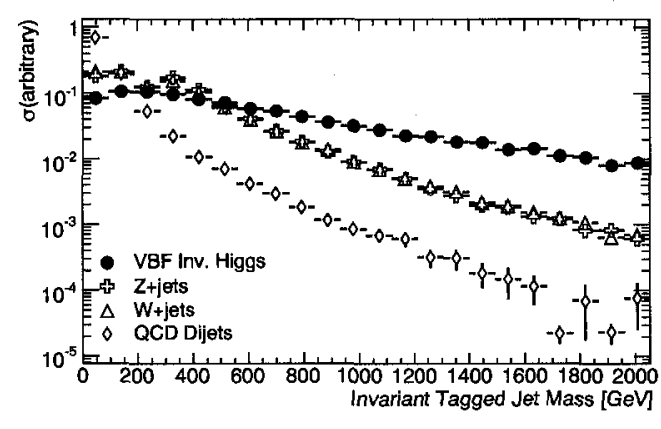

(a) Invariant mass of the tagged jets with no selection cuts. However, there was a 300 $\mathrm{GeV}$ cut on the $\mathrm{W} / \mathrm{Z}+$ jets background sample during the event generation as discussed in Section 9.3.3.

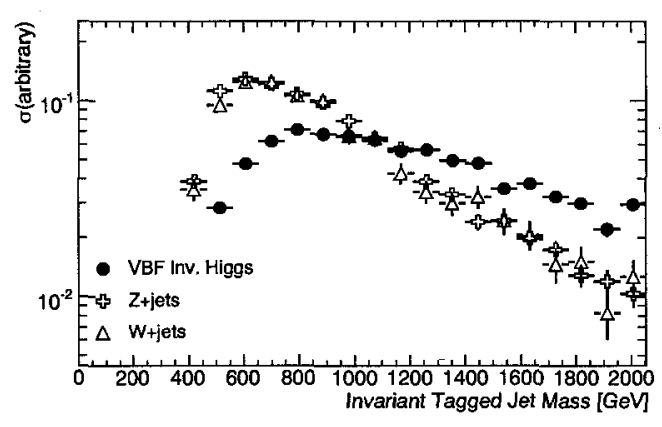

(b) Invariant mass of the tagged jets after the tagging jet selection cuts showing a clear separation between the signal and background events at $\sim 1200 \mathrm{GeV}$.

Figure 10.3: Cross-section in arbitrary units for the signal and background as a function of the invariant mass of the tagged jets. Signal events are for a $130 \mathrm{GeV}$ mass shown in bullets and background samples Z+jets, W+jets, and QCD dijets are shown in open crosses, triangles, and diamonds respectively. The distributions are normalized to unity. 
expected to be generated in these background events. The effectiveness of this cut for reducing the QCD dijet background can be seen in Figure 10.4(a). In addition, this cut helps remove $\mathrm{W} / \mathrm{Z}+$ jets events since the $\mathbb{E}_{T}$ for this background drops faster than for the signal. A cut of $\mathscr{E}_{T}>100 \mathrm{GeV}$ was selected based on the need to reduce the $\mathrm{W} / \mathrm{Z}+\mathrm{jet}$ backgrounds as can be seen in Figure10.4(b).

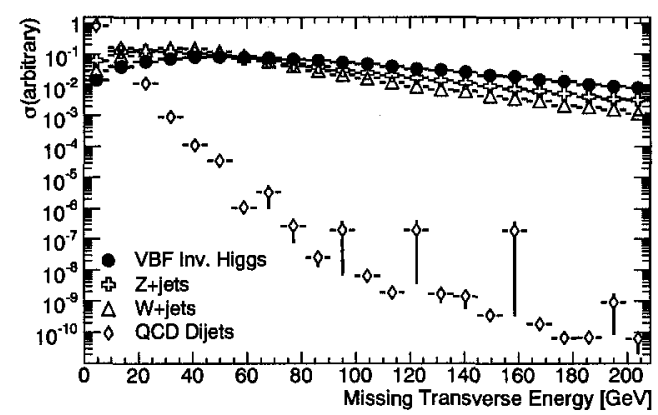

(a) No cuts

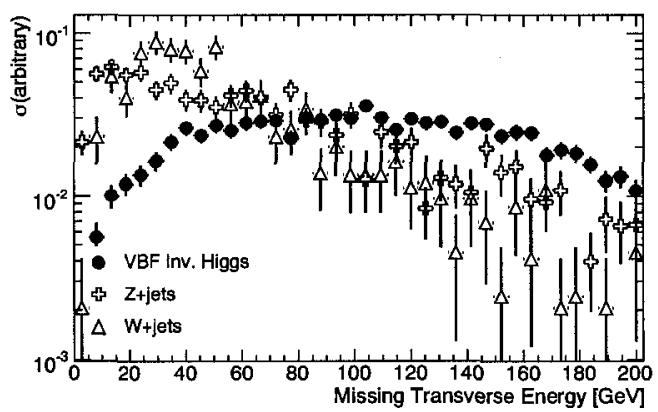

(b) After applying the jet tagging and invariant mass cuts.

Figure 10.4: Cross-section in arbitrary units for the signal and background as a function of $E_{T}$. Signal events are for a $130 \mathrm{GeV}$ mass shown in bullets and background samples $\mathrm{Z}+\mathrm{jets}, \mathrm{W}+\mathrm{jets}$, and QCD dijets are shown in open crosses, triangles, and diamonds respectively. The distributions are normalized to unity.

Lepton Veto: The $\mathrm{W}+$ jets and $\mathrm{Z}+$ jets backgrounds can be further reduced by rejecting events with any identified lepton. Events are rejected if they contain electrons or muons with a $p_{T}$ greater than $20 \mathrm{GeV}$. In a similar way, events with $\tau$-jets with a $p_{T}$ greater than $30 \mathrm{GeV}$ are rejected.

Missing Transverse Energy Isolation: In order to further reduce the QCD dijet background, a cut on the missing transverse energy isolation, $I$, was applied. This variable measures how close in $\phi$ the $\mathbb{E}_{T}$ is to the nearest of the two tagged jets and is defined as $I=\min \left[\phi\left(\mathbb{E}_{T}\right)-\phi\left(j_{1,2}\right)\right]$. Events with a small $I$ value are expected to be the result of mis-measured jets caused by dead material and cracks in the detector. Figure 10.5 illustrates that QCD dijets preferentially have small $I$. Therefore, events with $I<1$ radian are rejected. 


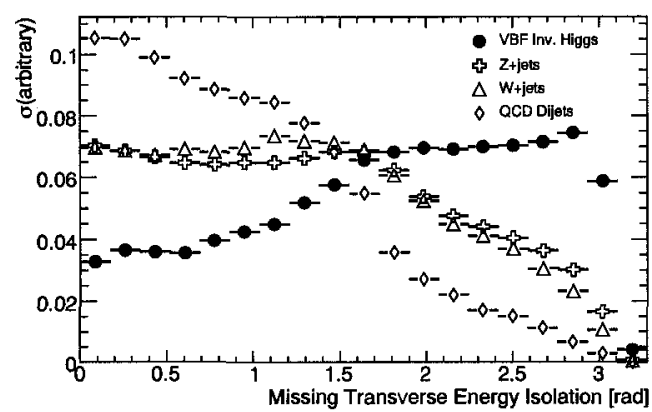

(a) No cuts

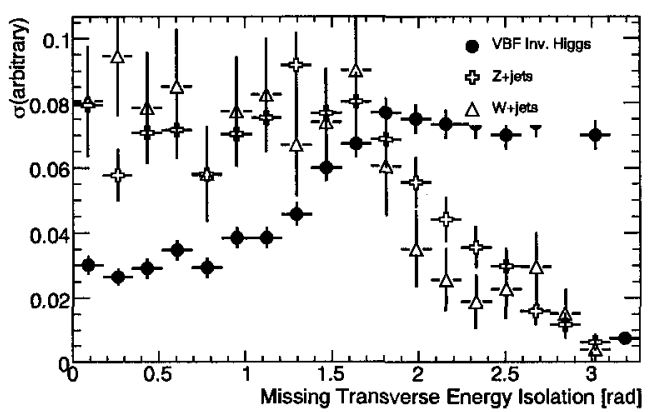

(b) After applying the jet tagging, invariant mass cuts, $\boldsymbol{E}_{T}$, and lepton veto cuts.

Figure 10.5: Cross-section in arbitrary units for the signal and background as a function of $I=\min \left[\phi\left(\mathscr{E}_{T}\right)-\phi\left(j_{1,2}\right)\right]$. Signal events are for a $130 \mathrm{GeV}$ mass shown in bullets and background samples $\mathrm{Z}+\mathrm{jets}, \mathrm{W}+\mathrm{jets}$, and QCD dijets are shown in open crosses, triangles, and diamonds respectively. The distributions are normalized to unity.

Central Jet Veto: A key aspect of the VBF Higgs search is the electroweak nature of the signal which allows background suppression because signal events do not have color flow between the interacting quarks at tree level. The irreducible background for the VBF invisible Higgs search comes from the production of $\mathrm{W}$ or $\mathrm{Z}$ in association with two high $p_{T}$ jets. The $\mathrm{W}+$ jets and $\mathrm{Z}+$ jets backgrounds include both electroweak and QCD terms, however, the cross-section is dominated by the QCD contribution since $\alpha_{s} \gg \alpha_{e m}$. Therefore, unlike the signal the majority of these background events have QCD radiation in the central region. The presence of the QCD radiation between the two tagging jets in principle provides a powerful tool to suppress this background by removing events with jet activity between the two tagged jets. This technique is generally referred to as a "Central Jet Veto". However, in addition to the hard interaction of interest, each event includes an Underlying Event (UE) which is not presently well modeled. For example, the ratio between Jimmy/Pythia for the average multiplicity due to the UE is $\sim 1.4-2.4$ depending on how the UE model was tuned [77]. Therefore, naively one would expect fewer Pythia events to survive this cut if the UE energy is shared throughout the jets, as Pythia produces fewer jets with larger $p_{T}$. Therefore, although there is a clear difference in the topology between the signal and background, the added contribution from the UE 
has a large effect on the efficiency of this cut. Ultimately the effect of the UE on the central jet veto efficiency will be measured using data and is beyond the scope of this thesis. In this thesis, the central jet veto required that there be no jets with $p_{T}$ greater than $30 \mathrm{GeV}$ for $|\eta|<3.2$.

Tagged Jet $\phi$ Separation: Unlike the signal VBF production, the $W+j e t s$ and Z+jets backgrounds can be produced by $q q \rightarrow q V$ process, shown in Figure 9.3(b), in which the second jet comes from a radiative process. As a consequence, the azimuthal angle between the two tagged jets, $\phi_{j j}$, between signal and background events of this kind is different, as shown in Figure 10.6. Figures 10.6(a) and Figure 10.6(b) show the tagged jet $\phi$ distribution without and with the previous offline cuts and clearly illustrates the discriminating power of this variable. The difference in $\phi_{j j}$ for the signal and background was used in the analysis presented in Section 10.4 by requiring $\phi_{j j}<1$ radian. In addition, the $\phi_{j j}$ variable distribution motivates the shape analysis presented in Section 10.5.

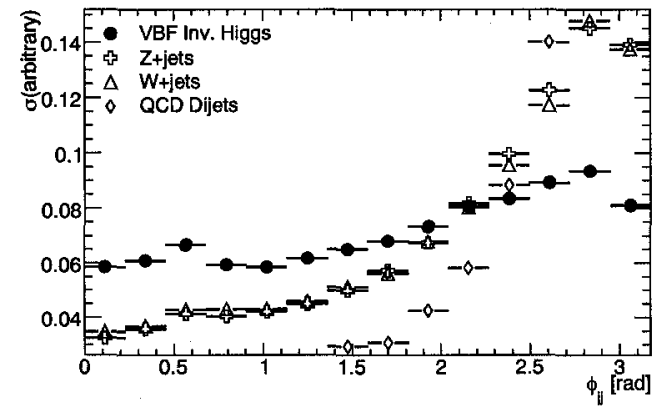

(a) No cuts

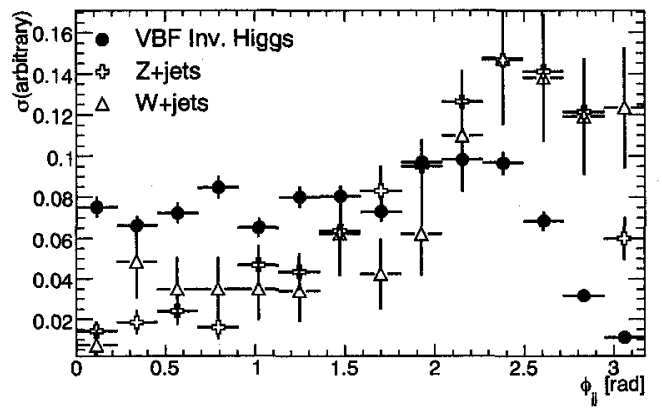

(b) After applying the jet tagging, invariant mass cuts, $E_{T}$, lepton veto, missing transverse energy Isolation, and central jet veto cuts.

Figure 10.6: Cross-section in arbitrary units for the signal and background as a function of the azimuthal angle between the tagged jets, $\phi_{j j}$. Signal events are for a $130 \mathrm{GeV}$ mass shown in bullets and background samples Z+jets, W+jets, and QCD dijets are shown in open crosses, triangles, and diamonds respectively. The distributions are normalized to unity. 


\subsection{Trigger}

Presented in this section is a summary of the level-1 trigger study. This study is based on the simulation of the ATLAS LVL1 trigger. It does not include the level-2 and event filter simulations because the high level trigger $\mathbb{F}_{T}$ algorithm was not fully implemented and there was no simulation of forward jets available for the high level trigger. The ATLAS LVL1 trigger simulation reproduces in detail the algorithms for $\mathbb{E}_{T}$ and jets implemented in the calorimeter system trigger hardware. Jets were classified into central jets $(|\eta|<3.2)$ and forward jets $(3.2<|\eta|<5)$. The $\eta$ break point of 3.2 corresponds to the physical boundary between the EndCap and Forward calorimeters.

The major triggering challenge for the VBF invisible Higgs analysis is to retain signal events while reducing the very large QCD background to an acceptable level. This problem is particularly acute for the first level trigger (level-1) which can easily be overwhelmed by the QCD background. The level-1 trigger system uses reduced granularity information and fast trigger specific algorithms in order to decide which events are of interest. This decision must be made within $\sim 2.5 \mu s$ which limits the level of sophistication that can be implemented in the level-1 software. It is this timing constraint and the reduced resolution which makes triggering on VBF invisible Higgs events very challenging. In addition, in order to add a new trigger item to the trigger menu for this channel, the proposed trigger item must not introduce more than a few $\mathrm{Hz}$ to the overall trigger rate.

The Monte-Carlo data used for this study was the $130 \mathrm{GeV}$ mass Higgs sample and the QCD dijet samples, as summarized in Sections 9.3.1 and 9.3.2, respectively. The $\mathrm{W}+\mathrm{jets}$ and $\mathrm{Z}+$ jets backgrounds were not considered since their contribution to the trigger rate is significantly smaller than the QCD dijets. The results of this study are shown in Table 10.1 for two luminosities, $10^{31} \mathrm{~cm}^{-2} \mathrm{sec}^{-1}$ and $10^{33} \mathrm{~cm}^{-2} \mathrm{sec}^{-1}$. The trigger items in the table 


\begin{tabular}{|l|r|r||r|}
\hline Trigger Menu & Acceptance[\%] & Rates[Hz] for $\mathcal{L}=10^{31}$ & Rates[Hz] for $\mathcal{L}=10^{33}$ \\
\hline XE60 & 99 & $5.00 \pm 1.30$ & $499 \pm 130$ \\
XE70 & 98 & $1.48 \pm 0.63$ & $148 \pm 63$ \\
XE80 & 96 & $0.58 \pm 0.14$ & $58 \pm 14$ \\
XE100 & 84 & $0.19 \pm 0.08$ & $19 \pm 8$ \\
XE120 & 70 & $0.07 \pm 0.05$ & $7 \pm 5$ \\
FJ20+XE70 & 79 & $0.89 \pm 0.57$ & $88 \pm 57$ \\
FJ23+XE70 & 78 & $0.87 \pm 0.57$ & $87 \pm 57$ \\
FJ25+XE70 & 76 & $0.83 \pm 0.56$ & $82 \pm 56$ \\
J20+XE70 & 83 & $1.45 \pm 0.63$ & $144 \pm 63$ \\
J23+XE70 & 83 & $1.43 \pm 0.63$ & $143 \pm 62$ \\
J25+XE70 & 82 & $1.42 \pm 0.62$ & $141 \pm 62$ \\
J23+XE100 & 73 & $0.19 \pm 0.08$ & $19 \pm 8$ \\
FJ23+XE100 & 66 & $0.02 \pm 0.01$ & $2 \pm 1$ \\
FJ23+J23+XE70 & 62 & $0.82 \pm 0.56$ & $82 \pm 57$ \\
FJ23+J23+XE100 & 55 & $0.02 \pm 0.01$ & $2 \pm 1$ \\
\hline
\end{tabular}

Table 10.1: Signal acceptance and trigger rates for the VBF invisible Higgs channel based on full ATLAS simulations. The trigger items are $\mathbb{E}_{T}(\mathrm{XE})$ central jet (J) and forward jet (FJ). The number following the menu object indicates the trigger threshold in $\mathrm{GeV}$. Both single and combined triggers are shown in this table. The values for a luminosity of $10^{33}$ are simply scaled and do not account for pile-up effects which are expected to be significant. Luminosities are in $\mathrm{cm}^{-2} \mathrm{~s}^{-1}$ units.

are $\mathscr{F}_{T}(\mathrm{XE})$, a central jet $(\mathrm{J})$, and a forward jet $(\mathrm{FJ})$ and the number following the trigger item indicates the trigger threshold in $\mathrm{GeV}$. These trigger rates are the raw rates for the specific trigger item and their statistical errors. It should be noted that the values for the higher luminosity are simply scaled and do not take into account pile-up effects ${ }^{1}$. Pile-up represents the processes which are not part of the hard scattering of interest, including the overlapping minimum bias events and remnant events for previous bunch crossings. As the luminosity increases, the pile-up effects become more acute since more contributions from remnant events will remain. It is presently not well understood how the pile-up effects scale with increased luminosity. One should note that within the trigger system events can be selected by multiple triggers. Therefore, the unique rate introduced by a new trigger item is expected to be less than the calculated raw rate. It is clear from this table that the trigger strategy will change depending on the luminosity. At low luminosi-

\footnotetext{
${ }^{1}$ A new study including pile-up effects should be performed once Monte-Carlo samples, which include high luminosity pile-up, are available.
} 
ties, $10^{31} \mathrm{~cm}^{-2} \mathrm{sec}^{-1}$, it is likely that a simple trigger based on $\mathbb{E}_{T}$ alone such as XE70 will be sufficient. This trigger item is already part of the ATLAS low luminosity trigger menu and is presently not pre-scaled. However, if the trigger rate turns out to be higher than expected, then a higher $\mathbb{E}_{T}$ threshold such as XE80 or XE100 can be used. As the luminosity increases or if backgrounds are worse than expected it will be necessary to use a combined trigger. From the results presented in Table 10.1, a trigger item based on the combination of $E_{T}$ and a forward or a central jet would be sufficient. However, these combinations provide a relatively small reduction in the trigger rates since there is a correlation between the jet energy and the $\mathbb{E}_{T}$ when high energy jets are mis-measured. Therefore, requiring the combination of one forward jet, one central jet and $\mathscr{E}_{T}$ is expected to suppress the QCD background to an acceptable rate albeit reducing the signal acceptance in the process. For high luminosity, $10^{33} \mathrm{~cm}^{-2} \mathrm{sec}^{-1}$, a conservative trigger item composed of a $E_{T}>100 \mathrm{GeV}$ and a forward and a central jet each with a $p_{T}>23 \mathrm{GeV}$ has been proposed. This trigger item has an acceptance rate for the signal of $55 \%$, as shown in Table 10.1. It should be stressed that a major source of uncertainty in trigger rates are from pileup effects which have not been considered for this analysis. Ultimately, adjustments will be required to select the optimum trigger based on the experimental rates observed at the LHC. For the analysis presented in this thesis, a trigger item composed of a $\mathbb{E}_{T}>100 \mathrm{GeV}$ and a forward and a central jet each with a $p_{T}>23 \mathrm{GeV}$ were used.

\subsection{Signal-To-Background Analysis}

This analysis uses the selection cuts summarized in Section 10.2. The signal significance is calculated based on the number of signal and background events that remain after all the cuts. The selection cuts along with the surviving cross-section after each cut are shown in Table 10.2 for a $130 \mathrm{GeV}$ Higgs mass and the three main backgrounds. Table 10.3 shows the effect of the cuts for the four Higgs mass points considered in this analysis. 
The first cut applied to the data simulates the effect of the LVL1 trigger with the most conservative menu option discussed in Section 10.3. This cut reduces the QCD dijet background rate by 7 orders of magnitude and by two orders of magnitude for the $\mathrm{W}+\mathrm{jets}$ and $Z+j e t s$ backgrounds. The jet tagging cut reduces all three backgrounds by a factor of 10 . The remaining cuts that have the largest impact on the QCD rate are the $\mathbb{E}_{T}$ cut and the $\mathbb{E}_{T}$ isolation cut which together reduce this background to a negligible level. The effect of these selection cuts on the $\mathrm{Z}+$ jets and $\mathrm{W}+$ jets backgrounds are less dramatic. The lepton veto reduces the $\mathrm{W}+\mathrm{jets}$ and $\mathrm{Z}+\mathrm{jets}$ by $\sim 36 \%$ and $\sim 3 \%$, respectively. The lepton veto cut removes few events in the $Z+j$ ets channel as the $\mathbb{F}_{T}$ cut has already removed most of the events for the $\mathrm{Z} \rightarrow \ell \ell$ background. The remaining $\mathrm{Z}+\mathrm{jet}$ events are dominated by the $\mathrm{Z} \rightarrow \nu \nu$ mode. In addition, the lepton veto cut does not remove all $\mathrm{W}+$ jet background events since lepton identification is only available for $|\eta|<2.5$. As a consequence, electrons and $\tau$-jets beyond $|\eta|>2.5$ are mis-identified as jets nearly $100 \%$ of the time. In a similar manner, muons beyond $|\eta|>2.5$ are not identified and result in fake $\mathbb{E}_{T}$.

The number of signal and background events after all the selection cuts in Tables 10.2 and 10.3 are used to calculate the $99 \%$ confidence level discovery potential for the invisible Higgs. This was done by calculating the number of signal events required to increase the total event count by a factor 2.33 times the error on the number of background events, as shown in Equation 10.1.

$$
2.33 \sigma_{B}=N_{S} \xi^{2}
$$

Here, $\sigma_{B}$ is the error on the number of background events, $N_{S}$ is the number of signal events, and $\xi^{2}$ is the sensitivity limit as defined in Section 8.4. Solving for $\xi^{2}$ provides the $99 \%$ confidence level discovery limit for the invisible Higgs. In the case where only statistical errors are considered, the error on the background is calculated using $\sigma_{B}=\sqrt{N_{B}}$. However, when the systematic errors are included the error on the background becomes $\sigma_{B}=\sqrt{N_{B}} \sqrt{1+\alpha^{2} N_{B}}$. Here, $\alpha$ is the fractional systematic error on the number of back- 


\begin{tabular}{|l|c|c|c|c|}
\hline Selection Cuts & Higgs 130 GeV & W+jets & Z+jets & QCD \\
\hline \hline Initial $\sigma(f b)$ & $3.93 \times 10^{3}$ & $1.24 \times 10^{6}$ & $4.08 \times 10^{5}$ & $1.91 \times 10^{13}$ \\
\hline LVL 1 Trigger & $2.71 \times 10^{2}(0.07)$ & $1.42 \times 10^{4}(0.01)$ & $6.31 \times 10^{3}(0.02)$ & $1.52 \times 10^{6}(0.00)$ \\
\hline+ Tagged jets & $1.47 \times 10^{2}(0.54)$ & $1.35 \times 10^{3}(0.10)$ & $6.16 \times 10^{2}(0.10)$ & $1.81 \times 10^{5}(0.12)$ \\
\hline$+M_{j j}$ & $1.11 \times 10^{2}(0.76)$ & $6.64 \times 10^{2}(0.49)$ & $3.76 \times 10^{2}(0.61)$ & $1.28 \times 10^{5}(0.71)$ \\
\hline$+\mathbb{E}_{T}>100 \mathrm{GeV}$ & $1.08 \times 10^{2}(0.97)$ & $4.70 \times 10^{2}(0.71)$ & $2.69 \times 10^{2}(0.72)$ & $2.84 \times 10^{3}(0.02)$ \\
\hline+ Lepton veto & $1.07 \times 10^{2}(1.00)$ & $3.01 \times 10^{2}(0.64)$ & $2.62 \times 10^{2}(0.97)$ & $2.76 \times 10^{3}(0.97)$ \\
\hline$+I>1$ rad & $9.60 \times 10^{1}(0.89)$ & $1.49 \times 10^{2}(0.49)$ & $2.11 \times 10^{2}(0.81)$ & $3.61 \times 10^{0}(0.00)$ \\
\hline+ Central jet veto & $8.93 \times 10^{1}(0.93)$ & $1.10 \times 10^{2}(0.74)$ & $1.32 \times 10^{2}(0.63)$ & $0.07 \times 10^{0}(0.02)$ \\
\hline$+\phi_{j j}<1$ rad & $4.50 \times 10^{1}(0.50)$ & $1.94 \times 10^{1}(0.18)$ & $4.21 \times 10^{1}(0.32)$ & $0.07 \times 10^{0}(1.00)$ \\
\hline
\end{tabular}

Table 10.2: Cross-section in fb for a $130 \mathrm{GeV}$ mass Higgs and background samples at each step of the selection process. Initial cross-section for $\mathrm{W} / \mathrm{Z}+\mathrm{jets}$ are quoted after MLM and VBF filter and NNLO correction. The first cut is the effect of the LVL1 trigger simulation with a $\mathbb{E}_{T}$ of $100 \mathrm{GeV}$, a central jet with $p_{T}>23 \mathrm{GeV}$ and a forward jet with $p_{T}>23 \mathrm{GeV}$. Numbers in parentheses are the efficiencies for each cut.

\begin{tabular}{|l|c|c|c|c|}
\hline Higgs Mass [GeV] & 100 & 130 & 200 & 250 \\
\hline \hline Initial $\sigma(f b)$ & 4630 & 3930 & 2410 & 1780 \\
\hline LVL 1 Trigger & 322 & 271 & 240 & 168 \\
\hline+ Tagged jets & 166 & 147 & 134 & 93 \\
\hline$+M_{j j}$ & 126 & 111 & 100 & 73 \\
\hline$+E_{T}>100 \mathrm{GeV}$ & 121 & 108 & 98 & 70 \\
\hline+ Lepton veto & 121 & 107 & 98 & 70 \\
\hline$+I>1$ rad & 108 & 96 & 86 & 63 \\
\hline+ Central jet veto & 94 & 89 & 79 & 59 \\
\hline$+\phi_{j j}<1$ rad & 43 & 45 & 39 & 30 \\
\hline
\end{tabular}

Table 10.3: Cross-section in fb for each signal mass at each step in the selection cuts. The first cut is the effect of the LVL1 trigger simulation with a $\mathbb{E}_{T}$ of $100 \mathrm{GeV}$, a central jet with $p_{T}>23 \mathrm{GeV}$ and a forward jet with $p_{T}>23 \mathrm{GeV}$. 
ground events. The systematic errors applied to this analysis are reported in Section 10.6 and lead to an $\alpha$ value of $23 \%$. The results of this analysis for an integrated luminosity of $30 \mathrm{fb}^{-1}$ are shown in Figure 10.7 by the black circles and triangles. In addition, open circles and triangles show the results obtained in a previous ATLAS analysis using a fast simulation technique [62]. For both analyses, the circles represent the sensitivity limit without systematic errors and the triangles represent the sensitivity limit with systematic errors. In addition, the Monte-Carlo statistics error was investigated and found to be negligible.

It can be seen that when the systematic errors are excluded, a significant improvement is observed with the analysis performed in this thesis over the previous ATLAS analysis using fast simulation [62] . The differences between the two results are believed to be due to the use of different event generators and their settings resulting in a difference in the cross-sections and the modeling of the UE. In the current analysis, the cross-sections were normalized to Next-to-Next-Leading Order (NNLO) in $\alpha_{s}$ with an uncertainty of $\sim 10 \%$ on the absolute cross-section. In contrast, the previous ATLAS analysis [62] used Leading Order (LO) in $\alpha_{s}$ which has an uncertainty of $\sim 300 \%$ on the absolute cross-section. In addition to the differences at the event generator level, there has been some improvements in the central jet veto cut algorithm.

When systematics are added, the sensitivity limit presented in this thesis increases by a factor of $\sim 1.5$ over the previous ATLAS analysis results [62]. This is related to the magnitude of the systematic errors associated with the offline reconstruction, particularly from the Jet Energy Scale (JES), which was not previously estimated. To show how dependent the sensitivity limit is on the JES, the sensitivity was calculated with a more optimistic JES of $\pm 5 \%$ for $|\eta|<3.2$ and $\pm 10 \%$ for $|\eta|>3.2$. When this is done, the sensitivity limit would improve from $\sim 70 \%$ to $\sim 50 \%$. 


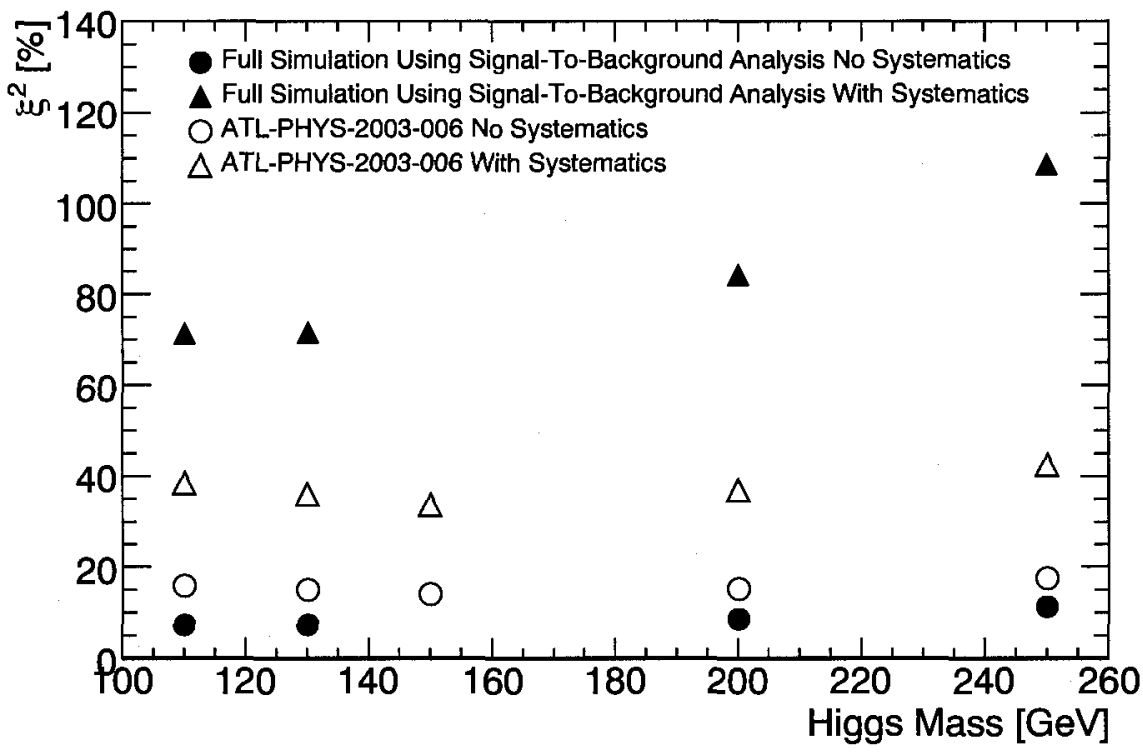

Figure 10.7: Sensitivity limit using the signal-to-background analysis for a $99 \%$ confidence level using an integrated luminosity of $30 \mathrm{fb}^{-1}$. All the cuts from Table 10.2 have been applied to the signal and background samples. Results with and without systematic errors are shown both for this analysis and an earlier analysis that used fast simulation events. In addition, the Monte-Carlo statistics error was investigated and found to be negligible. 


\subsection{Shape Analysis}

The shape analysis is motivated by a noticeable difference in the $\phi_{j j}$ distribution between the signal and the $\mathrm{W} / \mathrm{Z}+\mathrm{jets}$ background, as shown in Figure 10.6. This analysis involves taking the ratio of the number of events for $\left(\phi_{j j}<1\right)$ to all events as shown in Equation 10.2 .

$$
R=\frac{\int_{0}^{1} \frac{d \sigma}{d \phi_{j j}}}{\int_{0}^{\pi} \frac{d \sigma}{d \phi_{j j}}}=\frac{N_{B}^{1}+\xi^{2} N_{S}^{1}}{N_{B}^{\pi}+\xi^{2} N_{S}^{\pi}}
$$

Equation 10.2 can be re-written in the context of this analysis and expanded so that the first term depends only on the background, as shown in Equation 10.3.

$$
R=\frac{N_{B}^{1}}{N_{B}^{\pi}}\left[1+\xi^{2}\left(\frac{N_{S}^{1}}{N_{B}^{1}}-\frac{N_{S}^{\pi}}{N_{B}^{\pi}}\right)+\cdots\right]
$$

Here, $N_{B}^{1}$ and $N_{S}^{1}$ are the number of signal and background events within $\phi_{j j}<1$ and $N_{B}^{\pi}$ and $N_{S}^{\pi}$ are the number of events within the entire $\phi_{j j}$ range. The first term in Equation 10.3 provides the expected ratio for only the background. However, since the ratio between the signal and background are not the same, a non-zero value is expected for the second term. By taking the ratio, only the relative cross-section (i.e. shape) is needed. This has the benefit of reducing several systematic errors such as luminosity, energy scales, and absolute cross-section. It should be noted that the variation from the "background only" ratio dictates the sensitivity to new physics.

The shape analysis applies the selection cuts discussed in Section 10.2 up to the $\phi_{j j}$ cut. Therefore, the numbers in Table 10.2 are used in this analysis. The first term of Equation 10.3 is calculated to be $0.254 \pm 0.007$. Similarly to Section 10.4 , in order to determined the $99 \%$ confidence level discovery limit a variation of $2.33 \sigma_{R}$ is required. Therefore, the first order $\xi^{2}$ terms from Equation 10.3 are set to the required $99 \%$ confidence level sensitivity 
limit, that is $2.33 \sigma_{R}$, as shown in Equation 10.4.

$$
2.33 \sigma_{R}=\xi^{2}\left(\frac{N_{S}^{1}}{N_{B}^{1}}-\frac{N_{S}^{\pi}}{N_{B}^{\pi}}\right)\left(\frac{N_{B}^{1}}{N_{B}^{\pi}}\right)
$$

Solving for $\xi^{2}$ provides the $99 \%$ confidence level discovery limit for the invisible Higgs. The results of this analysis for an integrated luminosity of $30 \mathrm{fb}^{-1}$ are shown in Figure 10.8 by the black circles and triangles. The circles represent the sensitivity limit without systematic errors and the triangles represent the sensitivity limit with systematic errors. As with the signal-to-background analysis, the Monte-Carlo statistics error was investigated and found to be negligible. In addition, results from O. J. P.Eboli and D. Zeppenfeld [78] using data produced at the event generator level are shown by the open triangles. The results from O. J. P.Eboli and D. Zeppenfeld analysis were quoted for $95 \%$ confidence level, therefore a scale factor of 1.43 was applied to produce the $99 \%$ confidence level results presented in Figure 10.8. The largest systematic error for this analysis comes from the jet energy resolution and not the JES as with the previous analysis. This can be understood since the JES does not affect the $\phi_{j j}$ distribution but rather scales the individual components of the jet 4-vector equally. In contrast, the jet energy resolution does affect the $\phi_{j j}$ distribution since each individual component of the jet 4 -vector is affected. The results of this analysis show no increase in sensitivity over the signal-to-noise analysis when systematic uncertainty is included.

\subsection{Systematic Uncertainties}

In this section, two major types of systematic errors are considered. One of these errors arises from systematic uncertainties at the event generator level and the second from the reconstruction algorithms. 


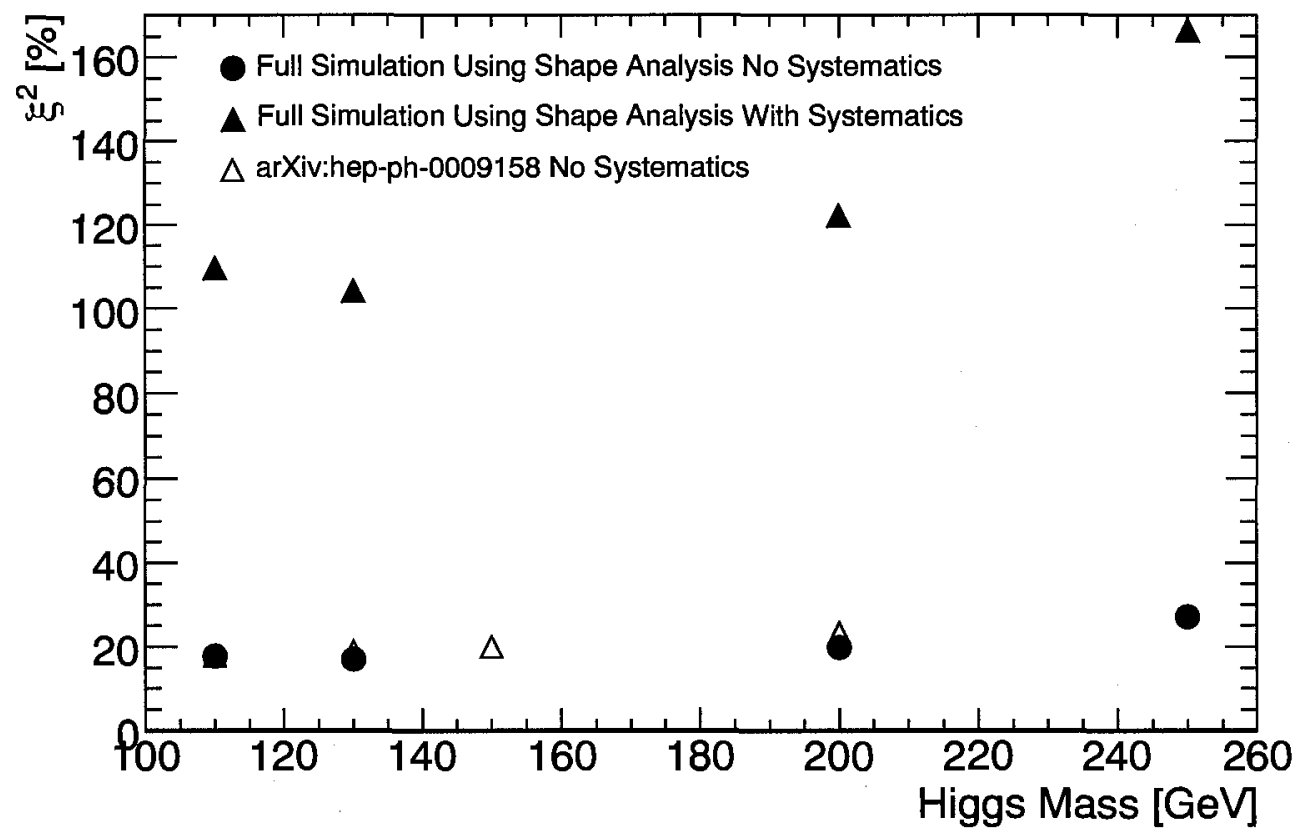

Figure 10.8: Discovery potential for an invisible Higgs at $99 \%$ confidence level via the VBF channel using shape analysis for an integrated luminosity of $30 \mathrm{fb}^{-1}$ with and without systematic errors. The black triangles and circles are the results from this analysis with and without systematic errors respectively. The open triangles are results from $\mathrm{O}$. J. P.Eboli and D. Zeppenfeld [78] using data produced at the event generator level. The results from O. J. P.Eboli and D. Zeppenfeld analysis were quoted for $95 \%$ confidence level, therefore a scale factor was applied to produce the $99 \%$ confidence level results presented in this figure. In addition, the Monte-Carlo statistics error was investigated and found to be negligible. 


\begin{tabular}{|l|c|c|}
\hline Selection Cuts & Herwig $130 \mathrm{GeV}$ & Pythia 130 GeV \\
\hline \hline Initial $\sigma(f b)$ & $3.93 \times 10^{3}(1.000)$ & $3.93 \times 10^{3}(1.000)$ \\
\hline Pre-Cut $\left(\mathscr{E}_{T}>80 \mathrm{GeV}\right)$ & $1.76 \times 10^{3}(0.448)$ & $1.78 \times 10^{3}(0.453)$ \\
\hline+ Tagged Jets & $4.07 \times 10^{2}(0.231)$ & $4.10 \times 10^{3}(0.230)$ \\
\hline$+M_{j j}$ & $2.45 \times 10^{2}(0.602)$ & $2.45 \times 10^{3}(0.598)$ \\
\hline$+\mathscr{F}_{T}>100 \mathrm{GeV}$ & $2.05 \times 10^{2}(0.837)$ & $2.14 \times 10^{3}(0.873)$ \\
\hline+ Lepton Veto & $2.05 \times 10^{2}(1.000)$ & $2.12 \times 10^{2}(0.991)$ \\
\hline$+I>1 \mathrm{rad}$ & $1.84 \times 10^{2}(0.898)$ & $1.80 \times 10^{2}(0.849)$ \\
\hline+ Central Jet Veto & $1.59 \times 10^{2}(0.864)$ & $1.07 \times 10^{1}(0.594)$ \\
\hline$+\phi_{j j}<1 \mathrm{rad}$ & $7.43 \times 10^{1}(0.467)$ & $4.93 \times 10^{1}(0.461)$ \\
\hline
\end{tabular}

Table 10.4: Comparison between Herwig and Pythia generated samples on the selection cut for the $130 \mathrm{GeV}$ Higgs mass. Cross-section results are quoted in $\mathrm{fb}$ and cut efficiency are in parenthesis.

\subsubsection{Event Generator Systematics}

Within the event generator there are several sources of systematic errors, such as the modeling of the Underlying Event (UE), renormalization scale, and kinematic shape distributions. The effect of the UE was investigated by considering two event generators, Jimmy and Pythia. These event generators were used because they model the UE differently. Table 10.4 shows the efficiency of each selection cut used in the VBF analysis for the two generators. The difference in cross-sections between the two samples are within $\sim 2 \%$ of each other for cuts up to the missing transverse energy isolation cut. However, after the central jet veto cut is applied, a factor of two fewer Pythia events survive resulting in a large difference between the two generators. This difference is consistent with the difference reported for the modeling of the UE between the two generator [77]. Since Jimmy produces less central jet activity, it provides a conservative estimate for the background. For this reason backgrounds generated with ALPGEN were interfaced with Herwig/Jimmy. In addition to the uncertainty in the UE there is a systematic error for the signal-to-noise analysis which arises from the LO absolute cross-section of the backgrounds. In order to reduce this uncertainty, the LO cross-section was normalized to the results calculated with the generator FEWZ $[79,80]$ at NNLO in $\alpha_{s}$ which is known to within $10 \%{ }^{2}$. How-

\footnotetext{
${ }^{2}$ Includes PDF and QCD scale uncertainty.
} 
ever, this correction does not change the uncertainty in the LO shape distributions used in the shape analysis. Therefore, a systematic error on the cross-section for the shape analysis was set at $\sim 10 \%$ based on the uncertainty of the LO shape distributions determined in a previous study [78]. In addition, the effect of the missing off-shell terms from the Alpgen background samples were checked. This was done using the $Z(\rightarrow \nu \nu)$ by adding a $\mathbb{E}_{T}$ contribution randomly generated by a Breit-Wigner distribution using the $\mathrm{Z}$ mass and width information. This study indicated that the effect of using a fixed mass boson was negligible.

\subsubsection{Reconstruction Systematics}

The systematic error for the reconstruction algorithm was investigated by considering the jet resolution and the JES. These quantities are assumed to have the largest contribution to this systematic error since they relate to both the measurement of the tagging jets and $\mathbb{E}_{T}$. In order to estimate the reconstruction systematic uncertainty, the ATLAS jet and $\mathbb{E}_{T}$ working group provided the expected performance for early data taking. For the transverse momenta of jets, the systematic error was introduced by smearing the $p_{T}$ of the jets using a Gaussian distribution with a width given by $\sigma(E)=0.45 \sqrt{E}$ for $|\eta|<3.2$ and $\sigma(E)=0.63 \sqrt{E}$ for $|\eta|>3.2$. The analysis sensitivity to the JES was investigated by shifting the overall scale by $\pm 7 \%$ for $|\eta|<3.2$ and $\pm 15 \%$ for $|\eta|>3.2$. In each case the $\mathbb{E}_{T}$ was then recalculated for the $\mathrm{x}$ and $\mathrm{y}$ components using Equation 10.5. This calculation was used to account for the change in the deposited energy within the events. Lepton reconstruction was also analyzed and found not to contribute to the final systematic error.

$$
\mathbb{E}_{T}{ }^{\text {new }}(x)=\mathbb{E}_{T}^{\text {original }}(x)-\sum p_{T}^{\text {original }}(x)+\sum p_{T}^{\text {new }}(x)
$$

The systematic error on the number of signal events was determined by varying the jet energy resolution and the jet energy scale and by looking at the variation in the number of signal events. These results are shown in the second column of Table 10.5. The same 
was done for the background events as shown in the third column of Table 10.5. These background systematic errors were used to calculate errors for the signal-to-noise analysis. For this analysis the systematic error is dominated by the JES since lowering the JES reduces the number of background events. In contrast, jet energy resolution was found to have a smaller systematic error. For the shape analysis, the same procedure was applied, however, the ratio of background events in the two parts of the $\phi_{j j}$ distribution was used instead of the number of events. The systematic errors for the shape analysis are shown in the fourth column of Table 10.5. From these results, the systematic error which dominates the shape analysis is the jet energy resolution. In contrast, changing the JES has little effect on the $\phi$ distribution.

In addition to the systematic error listed above, a $3 \%$ uncertainty was assigned to the luminosity for the signal-to-noise analysis. A summary of the systematic uncertainties considered are listed in Table 10.5. The overall systematic error for each analysis was calculated by adding the errors in quadrature with the exception of the jet energy scale. In this case, only the largest error from the jet energy scale was used for the overall systematic error calculation. The overall systematic error for the signal-to-noise analysis (shape analysis) was $22.8 \%(15.9 \%)$.

\subsection{Conclusion}

In this chapter, the following two analyses have been presented: a signal-to-background analysis and a shape analysis. Several potential level-1 trigger items were studied and a trigger item composed of a $\mathbb{E}_{T}>100 \mathrm{GeV}$ and a forward and a central jet each with a $p_{T}>23 \mathrm{GeV}$ were used to provide a robust trigger for high luminosity. A simulation of this trigger item was used as the first cut in the data selection process. The result for the signal-to-background analysis using $30 \mathrm{fb}^{-1}$ of data for a $130 \mathrm{GeV}$ mass Higgs was $\xi^{2} \sim 8 \%$ and $\sim 72 \%$ when including the systematic errors. Similarly, the results for the 


\begin{tabular}{|c|c|c|c|}
\hline \multirow{2}{*}{ Systematics } & \multirow{2}{*}{ Higgs $130 \mathrm{GeV}$} & \multicolumn{2}{|c|}{ Background } \\
\hline & & Signal-To-Noise & Shape \\
\hline Luminosity & \multicolumn{2}{|c|}{$3 \%$} & $\sim 0 \%$ \\
\hline Cross-section & $\mathrm{N} / \mathrm{A}$ & \multicolumn{2}{|l|}{$\sim 10 \%$} \\
\hline Event filter effects & $\mathrm{N} / \mathrm{A}$ & \multicolumn{2}{|l|}{$\sim 0.5 \%$} \\
\hline $\begin{array}{l}\text { Jets Resolution: } \\
\begin{array}{r}\sigma(E)=0.45 \sqrt{E} \text { for }|\eta|<3.2 \\
\sigma(E)=0.63 \sqrt{E} \text { for }|\eta|>3.2\end{array}\end{array}$ & $0.8 \%$ & $5.31 \%$ & $12.01 \%$ \\
\hline $\begin{array}{l}\text { Jets energy scale: } \\
\quad+7 \% \text { for }|\eta|<3.2 \\
\quad+15 \% \text { for }|\eta|>3.2\end{array}$ & $4.0 \%$ & $3.23 \%$ & $0.15 \%$ \\
\hline $\begin{array}{l}\text { Jets energy scale: } \\
\quad-7 \% \text { for }|\eta|<3.2 \\
-15 \% \text { for }|\eta|>3.2 \\
\end{array}$ & $10.0 \%$ & $19.52 \%$ & $2.77 \%$ \\
\hline Total & $11.2 \%$ & $22.8 \%$ & $15.9 \%$ \\
\hline
\end{tabular}

Table 10.5: Contributions to the systematic uncertainties for signal-to-background and shape analysis. The overall systematic error was calculated by summing the errors in quadrature with the exception of the jet energy scale. In this case only the largest error from the jet energy scale was used for the overall systematic error calculation.

shape analysis using $30 \mathrm{fb}^{-1}$ of data for a $130 \mathrm{GeV}$ mass Higgs sensitivity limit was $\sim 17 \%$ and $\sim 104 \%$ when including the systematic errors. From these results, it is clear that both analyses would benefit from studies focusing on reducing the uncertainty on the background. One such study, which was initially done using fast simulation [62], involves combining the measurements from the data with the predictions from Monte-Carlo. This is particularly important for determining the $Z \rightarrow \nu \nu$ background. However, this does not reduce the systematic error for the reconstruction algorithms which are the dominating uncertainty.

In order to validate any measurements which indicate an excess of background events, additional production channels should be used. There are several production channels that are under consideration within the ATLAS collaboration. A current study using fully simulated data for the $\mathrm{ZH}$ associated production modes has a preliminary result using 30 $\mathrm{fb}^{-1}$ of data for a $130 \mathrm{GeV}$ mass Higgs of $\xi^{2} \sim 90 \%$ including the systematic errors. 


\section{Chapter 11}

\section{Conclusion}

In this thesis two studies were performed in preparation for data taking at the ATLAS experiment. The first study investigated the intrinsic electron and hadron performance for the ATLAS FCal modules using data from the 2003 test beam. In the second study, the sensitivity to observe an invisible Higgs produced by the vector boson fusion production channel was performed using a fully simulated model of the ATLAS detector.

The performance of the FCal modules was measured for both electrons and hadrons. The main goals of the electron study were to determine the linearity and resolution for electrons as measured in the FCal 1 module. The linearity measurement allowed for a direct comparison with a simulated model of the readout electronics. This model will provide the initial electromagnetic response for the FCal modules during the early stages of ATLAS data taking. The measured electromagnetic response was $12.14 \pm 0.06 \mathrm{ADC} / \mathrm{GeV}$, which is in good agreement with the predicted value of $12 \mathrm{ADC} / \mathrm{GeV}$ from the simulation. In addition, the measured energy resolution for electrons was parameterized as $\sigma_{E} / E=(27.96 \pm 0.82) \% / \sqrt{E_{b}} \oplus(3.48 \pm 0.13) \%$, here $E_{b}$ is in $\mathrm{GeV}$, which is consistent with previously published FCal test beam results $[23,26]$. The hadron performance was investigated using two calibration schemes: flat weights and transverse weights. The latter calibration technique was developed to take advantage of the unique geometry of 
the FCal detector which is finely segmented perpendicular to the beam line. The energy resolution for hadrons using beam constrained flat weights and transverse weights was parameterized as $\sigma_{E} / E=(98.25 \pm 1.85) \% / \sqrt{E_{b}} \oplus(7.13 \pm 0.10) \%$ and $\sigma_{E} / E=$ $(72.7 \pm 1.5) \% / \sqrt{E_{b}} \oplus(2.85 \pm 0.28) \%$, respectively. These results show that the transverse weights reduce the stochastic term from $(98.25 \pm 1.85) \%$ to $(72.7 \pm 1.5) \%$ and the constant term from $(7.13 \pm 0.10) \%$ to $(2.85 \pm 0.28) \%$. In addition, the transverse weights provide a uniform energy response to within $3 \%$ for energies greater than $40 \mathrm{GeV}$.

The second part of this thesis, the sensitivity limit of the invisible Higgs via the VectorBoson-Fusion (VBF) mode using the ATLAS detector was investigated. A level 1 trigger study was performed and concluded that a trigger item requiring a missing transverse energy greater than $100 \mathrm{GeV}$ plus a central and a forward jet with transverse momentum greater than $23 \mathrm{GeV}$ was required to reduce the QCD background to an acceptable level. Two complementary sensitivity studies were performed: a signal-to-background analysis and a shape analysis. The signal-to-background analysis was based on selection cuts alone, while the shape analysis used the shape of the distribution of the azimuthal angle between the tagged jets. A study of the systematic uncertainty on the reconstruction algorithms indicated that the sensitivity limit for the invisible Higgs via the vectorboson-fusion production channel is dominated by the jet energy scale for the signal-tobackground analysis and the jet energy resolution for the shape analysis. The results from the signal-to-background (shape analysis) using $30 \mathrm{fb}^{-1}$ of data for a $130 \mathrm{GeV}$ mass Higgs show that it is possible to detect an invisible Higgs if the beyond the Standard Model cross-section is larger than $\sim 72 \%(\sim 104 \%)$ of the Standard Model cross-section at $99 \%$ confidence level assuming a $100 \%$ invisible Higgs branching ratio.

For the ATLAS FCal modules using data from the 2003 test beam, new studies to understand the effect of beams impacting the inner edge are currently underway. These studies will improve our understanding of the tails in the missing transverse energy distribution 
and provide valuable information needed to improve the jet energy scale in the FCal. As for the VBF invisible Higgs, a new analysis of the simulation using all three trigger levels is required to determine the event rate introduced by the proposed trigger item. 


\section{Bibliography}

[1] Computing technical design report. Technical Report CERN/LHCC/96-43, 1996.

[2] High-level trigger data aquisition and controls technical design report. Technical Report CERN/LHCC/2003-22, 2003.

[3] First-level trigger performance technical design report. Technical Report CERN/LHCC/98-14, 1998.

[4] Pixel detector performance technical design report. Technical Report CERN/LHCC/98-13, 1998.

[5] Muon spectrometer performance technical design report. Technical Report CERN/LHCC/97-22, 1997.

[6] Central solenoid performance technical design report. Technical Report CERN/LHCC/97-21, 1997.

[7] End-cap toroid performance technical design report. Technical Report CERN/LHCC/97-20, 1997.

[8] Barrel toroid performance technical design report. Technical Report CERN/LHCC/97-19, 1997.

[9] Magnet system performance technical design report. Technical Report CERN/LHCC/97-18, 1997. 
[10] Inner detector performance technical design report volume 1. Technical Report CERN/LHCC/97-16, 1997.

[11] Liquid argon calorimeter performance technical design report. Technical Report CERN/LHCC/96-41, 1996.

[12] Tile calorimeter performance technical design report. Technical Report CERN/LHCC/96-42, 1996.

[13] Calorimeter performance technical design report. Technical Report CERN/LHCC/96-40, 1996.

[14] Inner detector performance technical design report volume 2. Technical Report CERN/LHCC/97-17, 1997.

[15] L. Di Ciaccio. Test beam results on the ATLAS electromagnetic calorimeter. Eur. Phys. J., C33:s1005-s1107, 2004.

[16] G. Sauvage. The ATLAS electromagnetic calorimeter: Construction, commissioning and selected test beam results. Nucl. Phys. Proc. Suppl., 150:98-101, 2006.

[17] G. Aad and et al. The ATLAS Experiment at the CERN Large Hadron Collider. JINST, 2008.

[18] Richard Wigmans. On the energy resolution of uranium and other hadron calorimeters. Nucl. Instrum. Meth., A(259):389-429, 1987.

[19] C. Amsler, D. Asner, and et. al. Review of particle physics. Journal of Physics G, 33, 2006.

[20] Richard Fernow. Introduction to experimental particle physics. Cambridge University Press, 1992.

[21] T. A. Gabriel, Donald E. Groom, P. K. Job, N. V. Mokhov, and G. R. Stevenson. Energy dependence of hadronic activity. Nucl. Instrum. Meth., A(338), 1994. 
[22] Richard Wigmans. Calorimetry : Energy Measurement in Particle Physics. Clarendon Press, 2000.

[23] J.C. Armitage and et al. Electron signals in the forward calorimeter prototype for atlas. JINST 2 P11011, 2007.

[24] W.E. Cleland and E.G. Stern. Signal processing considerations for liquid ionization calorimeters in a high rate environment. Nucl. Instrum. Meth. A, 338, 1994.

[25] L. W. Nagel and D. O. Pederson. Spice (simulation program with integrated circuit emphasis). University of California, Berkeley, April 1973.

[26] J.P. Archambault and et al. Energy calibration of the atlas liquid argon forward calorimeter. JINST 3 P02002, 2008.

[27] S. L. Glashow. Partial Symmetries of Weak Interactions. Nucl. Phys., 22:579-588, 1961.

[28] Steven Weinberg. A Model of Leptons. Phys. Rev. Lett., 19:1264-1266, 1967.

[29] Abdus Salam. Weak and Electromagnetic Interactions. Originally printed in *Svartholm: Elementary Particle Theory, Proceedings Of The Nobel Symposium Held 1968 At Lerum, Sweden*, Stockholm 1968, 367-377.

[30] S. Dawson. Introduction to the physics of Higgs bosons. 1994. hep-ph/9411325.

[31] Chris Quigg. The electroweak theory. 2002. hep-ph/0204104.

[32] R. Barate and et al. Search for the standard model Higgs boson at LEP. Phys. Lett., B565:61-75, 2003.

[33] The LEP Collaborations: Aleph collaboration, DELPHI Collaboration, L3 Collaboration, OPAL Collaboration, and the LEP Electroweak Working Group. Precision electroweak measurements and constraints on the standard model, 2007.

[34] Chris Quigg. Spontaneous Symmetry Breaking as a Basis of Particle Mass. Rept. Prog. Phys., 70:1019-1054, 2007. 
[35] Z. Kunszt, S. Moretti, and W. James Stirling. Higgs production at the LHC: An update on cross sections and branching ratios. Z. Phys., C74:479-491, 1997.

[36] Stephen P. Martin and James D. Wells. Motivation and detectability of an invisiblydecaying higgs boson at the fermilab tevatron. Phys. Rev., D60, 1999.

[37] G. Belanger, F. Boudjema, A. Cottrant, R. M. Godbole, and A. Semenov. The mssm invisible higgs in the light of dark matter and g-2. Phys. Lett., B519, 2001.

[38] Raghavendra Srikanth Hundi, Biswarup Mukhopadhyaya, and Andreas Nyffeler. Invisibly decaying higgs boson in the littlest higgs model with t-parity. arXiv:0707.1591 [hep-ph], July 2007.

[39] T. Binoth and J. J. van der Bij. Influence of strongly coupled, hidden scalars on higgs signals. Z. Phys., C75, 1997. hep-ph/9608245.

[40] T. Binoth and J. J. van der Bij. The stealthy higgs model at future linear colliders. hep-ph/9908256, August 1999.

[41] M. Battaglia, D. Dominici, J. F. Gunion, and J. D. Wells. The invisible higgs decay width in the add model at the lhc. hep-ph/0402062, 2004.

[42] Daniele Dominici. Probing extra dimensions through the invisible higgs decay. Czech. J. Phys., 55:B627-B634, B627-B634. hep-ph/0503216.

[43] K. Belotsky, D. Fargion, M. Khlopov, R. Konoplich, and K. Shibaev. Invisible higgs boson decay into massive neutrinos of 4th generation. Phys. Rev., D68, 2003.

[44] S. S. Bulanov, V. A. Novikov, L. B. Okun, A. N. Rozanov, and M. I. Vysotsky. Zlineshape versus 4th generation masses. YAD.FIZ., 66:2219, 2003.

[45] V. A. Novikov, L. B. Okun, A. N. Rozanov, and M. I. Vysotsky. Mass of the higgs versus fourth generation masses. PISMA ZH.EKSP.TEOR.FIZ., 76:158, 2002. 
[46] Nima Arkani-Hamed, Savas Dimopoulos, and G. R. Dvali. The hierarchy problem and new dimensions at a millimeter. Phys. Lett., B429:263-272, 1998.

[47] Anindya Datta, Katri Huitu, Jari Laamanen, and Biswarup Mukhopadhyaya. Invisible Higgs in theories of large extra dimensions. 2005. hep-ph/0505104.

[48] Isabel Josa Mutuberria. Searches for Higgs bosons beyond the Standard Model at LEP (Part B): Fermiophobia, anomalous couplings, invisible and charged Higgs. Prepared for International Europhysics Conference on High- Energy Physics (HEP 2001), Budapest, Hungary, 12-18 Jul 2001.

[49] Pauline Gagnon. Invisible higgs boson decays in the $\mathrm{zh}$ and wh channels. $A T L-$ PHYS-PUB-2005-011, 2005.

[50] Frank Meisel, M. Duhrssen, M. Heldmann, and K. Jakobs. Study of the discovery potential for an invisibly decaying higgs boson via the associated zh production in the atlas experiment. ATL-PHYS-PUB-2006-009, 2006.

[51] Borut Paul Kersevan, Maciej Malawski, and Elezbieta Richter-Was. Prospects for observing invisibly decaying higgs boson in the $t \bar{t}$ h production at lhc? ATL-PHYS2003-028, 2003.

[52] G. Corcella and et al. Herwig6: an event generator for hadron emission reactions with interfering gluons. hep-ph/0011363, 2000.

[53] G. Corcella and et al. Herwig 6.5 release note. hep-ph/0210213v2, 2005.

[54] J. M. Butterworth, Jeffrey R. Forshaw, and M. H. Seymour. Multiparton interactions in photoproduction at hera. Z. Phys., C72:637-646, 1996.

[55] J.M. Butterworth and M. H. Seymour. Jimmy4: Multiparton interactions in herwig for the lhc. http:/ / projects.hepforge.org/jimmy/draft20051116.ps.

[56] Torbjorn Sjostrand, Stephen Mrenna, and Peter Skands. Pythia 6.4 physics and manual. hep-ph/0603175, 2006. 
[57] Michelangelo L. Mangano, Mauro Moretti, and Roberto Pittau. Multijet matrix elements and shower evolution in hadronic collisions: $w b \bar{b}+(\mathrm{n}) \mathrm{jets}$ as a case study. Nucl. Phys., B632:343-362, 2002.

[58] M.L. Mangano, M. Moretti, F. Piccinini, R. Pittau, and A. Polosa. Alpgen, a generator for hard multiparton processes in hadronic collisions. JHEP, 07:001, 2003.

[59] Stefan Hoche et al. Matching parton showers and matrix elements. hep-ph/0602031, 2006.

[60] Bruce Mellado, Leandro Nisati, Daniela Rebuzzi, Stefano Rosati, Guillaume Unal, and Sau Lan Wu. Higgs production cross-sections and branching ratios for the atlas higgs working group. COM-PHYS-2007-024, 2007.

[61] Arthur M. Moraes. Tuning pythia 6.403 to the underlying event. ATLAS Monte-Carlo Group Meetings, October 2006.

[62] Lionel Neukermans and Beniamino Di Girolamo. Observing an invisble decaying higgs in atlas via vector boson fusion. ATL-PHYS-2003-006, 2003.

[63] Guilherme Nunes Hanninger. Atlas level-1 trigger studies for the invisibly decaying higgs boson producted in vector boson fusion. Master's thesis, University of Bonn, 2007.

[64] Junichi Tanaka and S.Asai. Vbf filter validation and systematic study of alpgen $z \rightarrow$ ee. ATLAS Monte-Carlo Group Meetings, November 2006.

[65] S. Agostinelli and et al. Geant4-a simulation toolkit. Nuclear Instruments and Methods $A,(506): 250-303,2003$.

[66] J. Allison and et al. Geant4 developments and applications. IEEE Transactions on Nuclear Science, 53(1):270-278, 2006.

[67] Elzbieta Richter-Was. Atlfast 2.0 a fast simulation package for atlas. ATL-PHYS-98$131,1998$. 
[68] C. Cojocaru and et al. Hadronic calibration of the atlas liquid argon end-cap calorimeter in the pseudorapidity region $1.6<|\eta|<1.8$ in beam tests. Nucl. Instrum. Meth. A, 531(481), 2004.

[69] G.C. Blazey and et al. Run 2 jet physics. hep-ex/0005012 v2, May 2002.

[70] H1 Collaboration. Nucl. Instrum. Meth. A, 386:348, 1997.

[71] K. Cranmer, A. Farbin, and A. Shibata. Eventview - the design behind an analysis framework. ATL-SOFT-PUB-2007-008, December 2007.

[72] Atlas detector and physics performance volume 1. Technical Report CERN/LHCC/99-14, 1999.

[73] Frederic Derue and Cedric Sefon. Electron/jet seperation with dc1 data. ATL-PHYSPUB-2005-016, 2005.

[74] F. Tarrade. Reconstruction and identification of $\tau$-decays in atlas. ATL-COM-PHYS2006-085, page 6, 2006.

[75] F. Tarrade. Tau identification at atlas: importance, method and confrontation with monte carlo and test beam. ATL-COM-PHYS-2006-084, page 5, 2006.

[76] A.-C. Le Bihan and et al. Identification of hadronic $\tau$ decays with atlas detector. ATL-COM-PHYS-2007-066, page 160, 2007.

[77] C.M.Buttar, J.M.Butterworth, R.D.Field, C.Group, G.Gustafson, S.Hoeche, F.Krauss, A.Moraes, M.H.Seymour, A.Schalicke, P.Szczypka, and T.Sjostrand and. The underlying event. HERA and the LHC: A workshop on the implications of HERA for LHC physics, 2007.

[78] O. J. P.Eboli and D. Zeppenfeld. Observing an invisible higgs boson. arXiv:hepph/0009158 v1, September 2000. 
[79] Kirill Melnikov and Frank Petriello. Electroweak gauge boson production at hadron colliders through O(alpha(s)**2). Phys. Rev., D74:114017, 2006.

[80] Kirill Melnikov and Frank Petriello. The $\mathrm{W}$ boson production cross section at the LHC through O(alpha(s)**2). Phys. Rev. Lett., 96:231803, 2006. 